\title{
TELLING OUR STORIES ON THE WEB: CANADIAN ENGLISH-LANGUAGE WEB SERIES AND THE PRODUCTION OF CULTURE ONLINE
}

\author{
by
}

\author{
Emilia Zboralska
}

Master of Arts, University of Toronto, Toronto, Ontario, Canada, 2010

Bachelor of Arts, University of Toronto, Toronto, Ontario, Canada, 2009

\begin{abstract}
A dissertation presented to Ryerson University and York University in partial fulfillment of the requirements for the degree of Doctor of Philosophy in the Joint Graduate Program in Communication and Culture
\end{abstract}

Toronto, Ontario, Canada, 2018

(C)Emilia Zboralska, 2018 


\section{AUTHOR'S DECLARATION FOR ELECTRONIC SUBMISSION OF A DISSERTATION}

I hereby declare that I am the sole author of this dissertation. This is a true copy of the dissertation, including any required final revisions, as accepted by my examiners.

I authorize Ryerson University to lend this dissertation to other institutions or individuals for the purpose of scholarly research.

I further authorize Ryerson University to reproduce this dissertation by photocopying or by other means, in total or in part, at the request of other institutions or individuals for the purpose of scholarly research.

I understand that my dissertation may be made electronically available to the public. 


\title{
TELLING OUR STORIES ON THE WEB: CANADIAN ENGLISH-LANGUAGE WEB SERIES AND THE PRODUCTION OF CULTURE ONLINE
}

\author{
Emilia Zboralska \\ Doctor of Philosophy \\ Joint Graduate Program in Communication and Culture \\ Ryerson University and York University \\ 2018
}

\begin{abstract}
This dissertation presents the first critical scholarly analysis of the Canadian
\end{abstract}

English-language scripted web series industry, its cultural practices, industrial dynamics and texts. Through in-depth interviews with 48 individuals active in the production of Canadian online scripted content, participant observation, and a benchmark quantitative analysis of gender and race in key creative roles in 175 seasons of Canadian web series, the dissertation investigates the web as an alternative space for Canadian scripted audiovisual content, and the actors and forces that have shaped and are shaping its development, including its emergent patterns of inclusion.

By developing a novel theoretical framework that combines the critical political economy of communication with entrepreneurship studies, the dissertation is able to mediate effectively between structure and agency to reveal how Canadian web series creators are interpreting, internalizing and resisting larger institutional dynamics and discourses in their cultural practices and texts. Through their entrepreneuring, Canadian web creators are reacting to a variety of rigidities within the contextual 
dimensions in which they are embedded, including the absence of meaningful opportunities to practice their crafts, the persistence of networks of exclusion, and inaccurate or missing on-screen representations of themselves or others in mainstream media. Through their work, they desire to achieve freedom from these constraints. The challenge of disrupting the status quo is then revealed through an examination of the domestic and extra-national structural factors that act as impediments to their agency.

The dissertation problematizes ideas of participation and access on the web, and introduces new conceptual terminology through the Participatory Culture Paradox, to encapsulate the contradictory set of relations that on the one hand, enables creators' activities in the online space, and at the same time, constrains their capacity to find audiences and monetize their work.

The findings here demonstrate that as much as internet-based distribution has expanded opportunities for participation for regular users, who you are, and where you are based, continue to be salient mediators of both participation and success in the development of professional scripted screen careers in the digital age.

The dissertation culminates in actionable priorities for Canadian policy that aim at change. 


\section{Acknowledgements}

There is no possible way for me to express the magnitude of my gratitude to the many brilliant and caring people who shared their energy, time, knowledge, understanding, resources and patience in order to help me on this path. I could not have gotten here without you.

Thank you to my supervisory committee, Dr. Charles Davis, Dr. David Skinner and Dr. Nicole Neverson, for continuously supporting me and pushing me to go further. I am indebted to your expertise and kindness. Charles, I cannot thank you enough for all that you have done to expand my thinking and growth as a scholar and researcher throughout the years including your contributions of time, ideas, and funding. I genuinely could not have gotten to the finish line without your guidance and support. It's all done, including the words!

I am just so fortunate to have been surrounded by so many wonderful people throughout this experience. To the entire Faculty of Communication and Design community at Ryerson, thank you for embracing and encouraging me. Dr. Irene Berkowitz and Donna Ratchford, thank you for being such inspirational and fearless women, mentors, leaders and friends. Dr. Jeremy Shtern, thank you for being supportive and always ready to listen and offer advice. You've gotten me out of several pickles! 
Maggie Reid, I was so lucky to find someone I mesh with creatively and who shares my passion for pushing the boundaries of Canadian storytelling. We've been on so many adventures already and they are only beginning. Yanna Chevtchouk, it was a privilege sharing the teaching stage with you. I've learned so much just by watching you in action. You two phenomenal women are next!

Thank you to the Independent Web Creators of Canada for welcoming me, and inspiring me. To the talented creators and individuals who shared their stories and perspectives for this work, thank you so much for your time, enthusiasm and candour.

To my other friends and family, I love all of you, and I know I've missed a lot. Thank you for your patience and for not forgetting me! Mamcia, thank you for always supporting me, and being there for me even though I'm "becoming the wrong kind of doctor". I would be nowhere without your unconditional love and delicious cooking. Tata, thank you for your endless animal videos. Evan, I feel like you're owed an honorary doctorate for putting up with me these last five years. This is as much yours as it is mine. Thank you for always being willing to listen, contribute and debate ideas in the middle of the night. I could not have done this without you.

Finally, this work would not have been possible without support from the Social Sciences and Humanities Research Council of Canada, the Creating Digital Opportunities Project, the Canadian Media Research Consortium, the Ontario 
Graduate Scholarship, and the Edward S. Rogers Sr. Graduate School Fellowship, all of which are gratefully acknowledged. 


\section{Table of Contents}

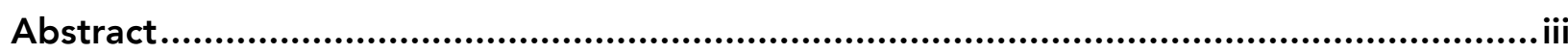

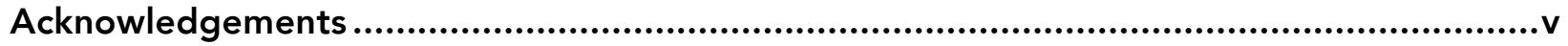

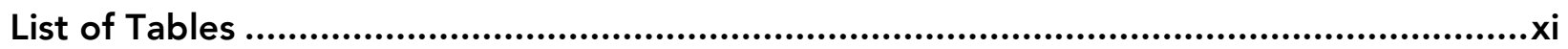

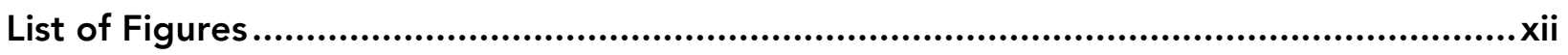

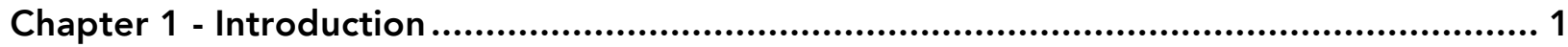

Chapter 2 - Setting the Stage: Theory and Literature Review ................................... 21

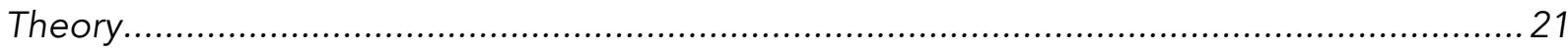

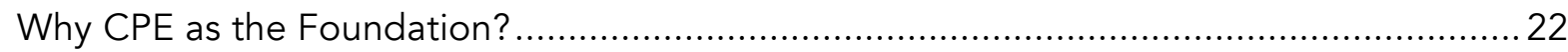

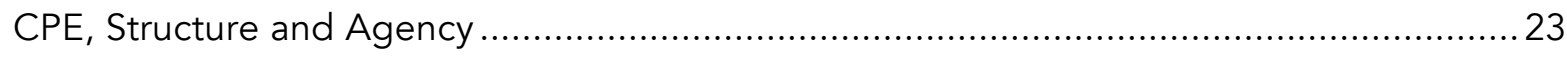

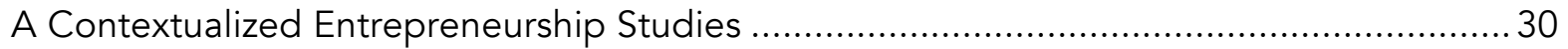

CPE and Entrepreneurship Studies: An Unlikely Marriage ............................................... 37

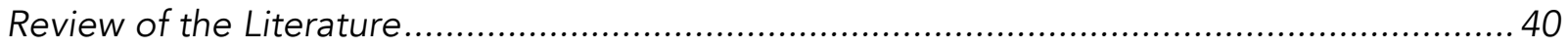

Critical Political Economy of the Traditional Television System ......................................... 40

The Internet, 'Participation' and its Spaces and Players........................................... 42

Production of Scripted, Serialized Content for the Web .............................................. 50

Canadian Production of Scripted, Serialized Content for the Web ................................. 57

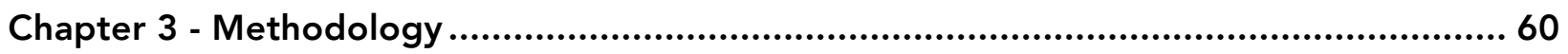

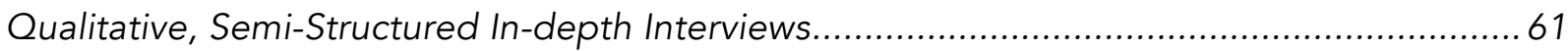

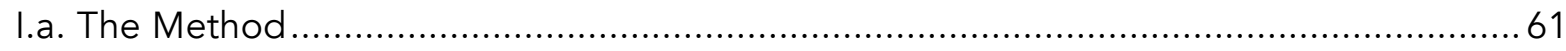

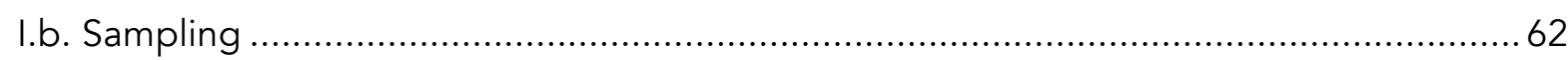

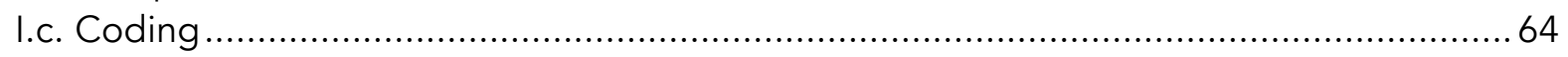

Quantitative Gender and Diversity Analysis of Key Roles in Web Series ............................66

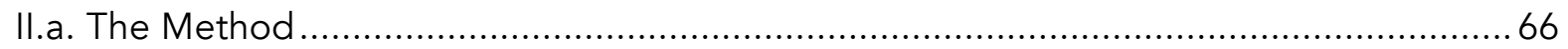

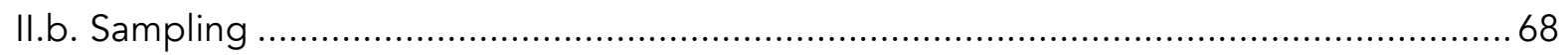

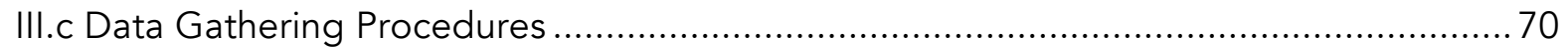

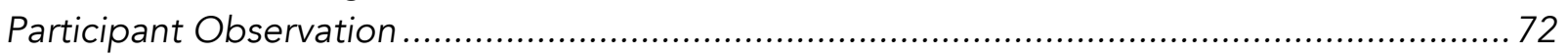

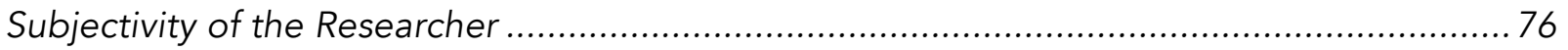

Chapter 4 - The World in Which They Create: The Spatial, Temporal, Institutional and Social Dimensions of Screen Production and Distribution in Canada .............................. 79

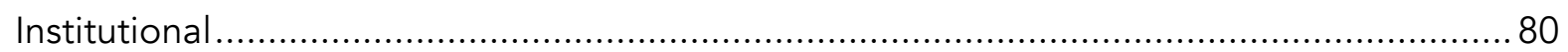

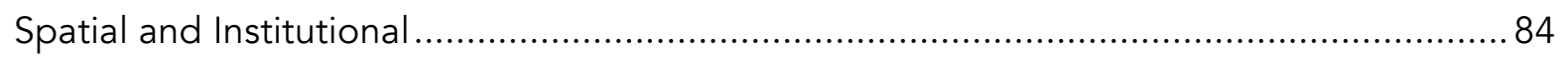

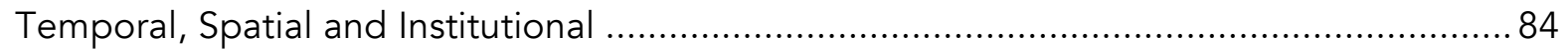

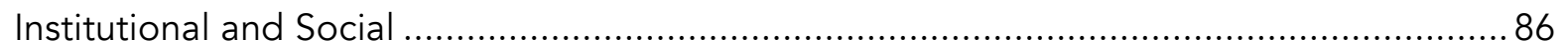

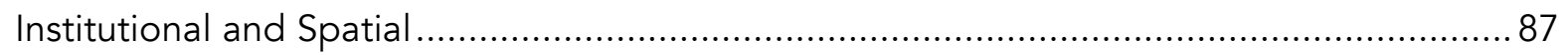

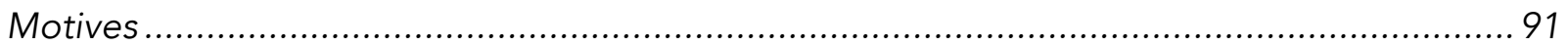

i. To Overcome Canadian Industry Endemic Problems .................................................... 92

ii. Identity, Marginalization and Social Good ............................................................... 100 


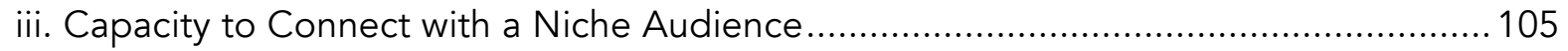

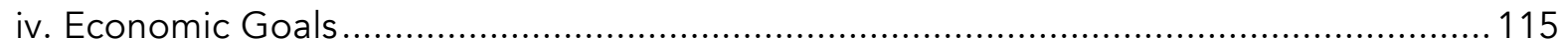

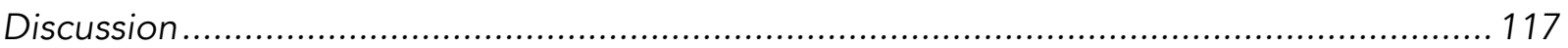

Chapter 5 - Identity and Participation and the Intensified Web Series Model of Production

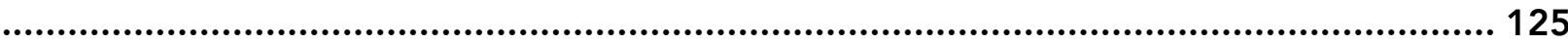

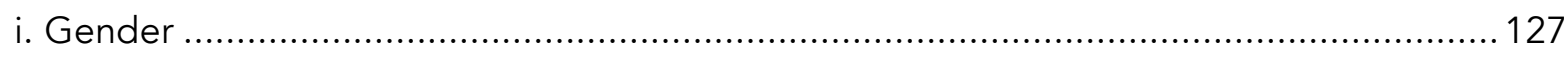

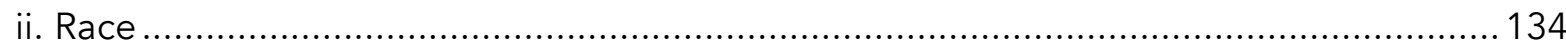

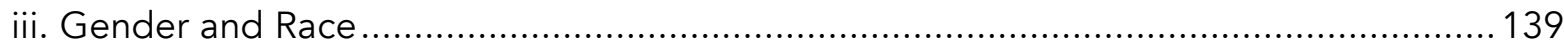

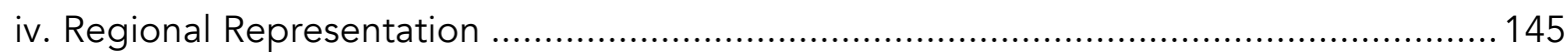

v. Regional Representation and Race: Ontario ........................................................ 147

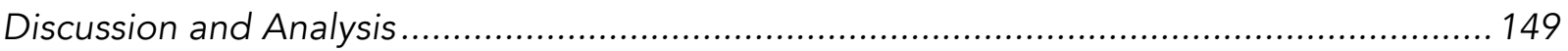

General Creative Industries Model of Production ........................................................... 153

Intensified Model of Production in Web Series........................................................... 158

Chapter 6 - Barriers and Challenges from Outside of the Canadian System ................. 177

Achieving Key Success Metrics: Audiences and Economics ....................................... 178

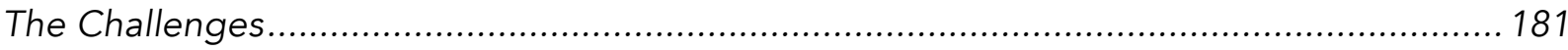

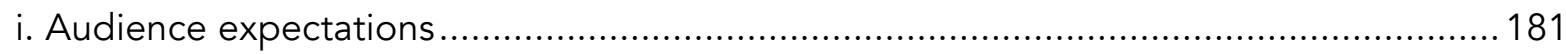

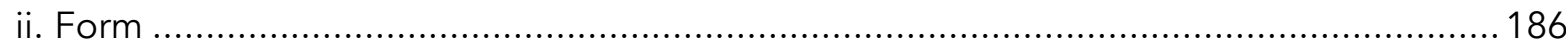

iii. Digital Dimes, Analog Dollars and Digital Efficiency............................................... 192

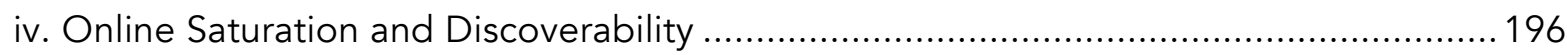

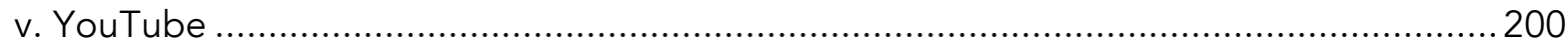

vi. The Cooling of the 'Wild West' ................................................................................. 208

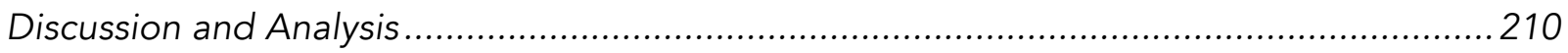

Chapter 7 - Barriers and Challenges from Within the Canadian System ....................... 215

The Challenges...................................................................................................... 215

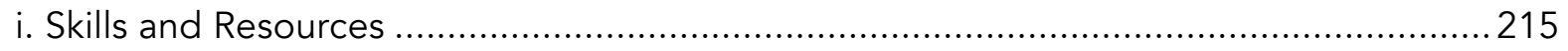

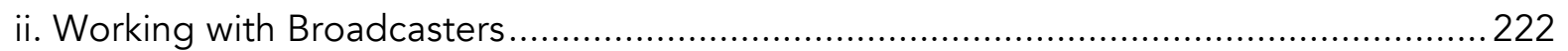

iii. Broadcaster Digital Leadership .................................................................. 225

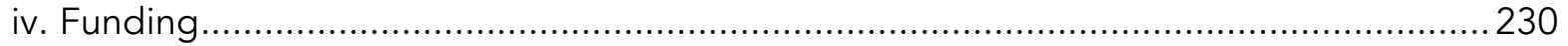

v. Hiring Canadian Actors ....................................................................................... 236

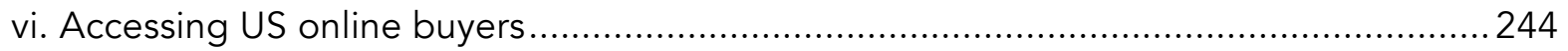

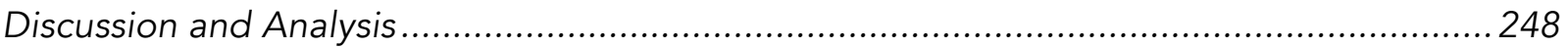

Chapter 8 - Conclusion: Encouraging Canadian Screen Industry Change by Empowering

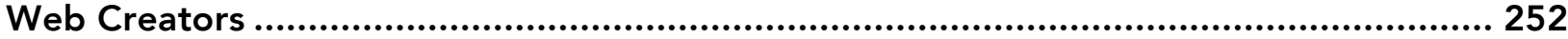

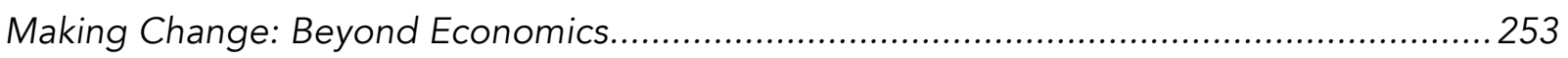

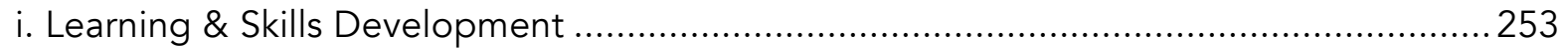

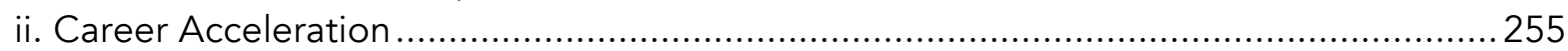

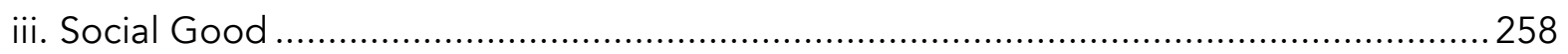

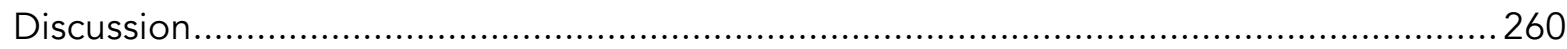

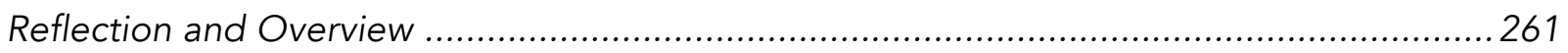


Harnessing the Potential: Policy and Program Recommendations ...............................268

i. New Sources of Funding for Canadian Content ........................................................... 274

ii. Designing Funding Programs that Encourage New Voices .................................... 279

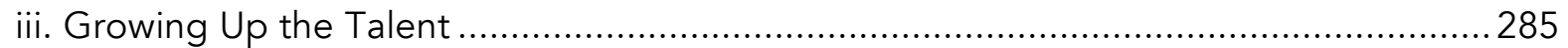

iv. Monitoring Diversity in Canadian Original Production ...........................................290

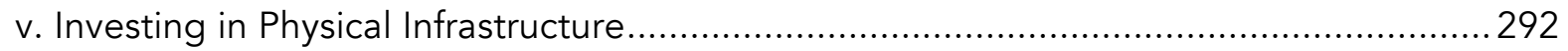

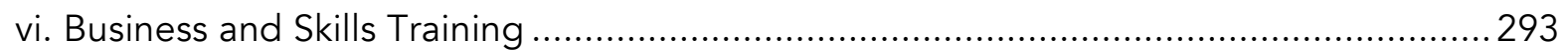

vii. An Expanded Role for the Public Broadcaster..................................................... 297

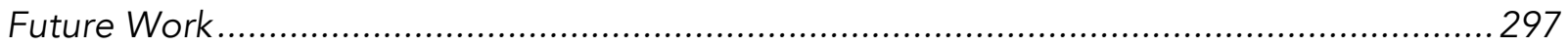

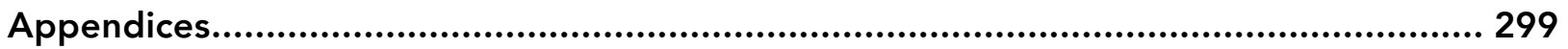

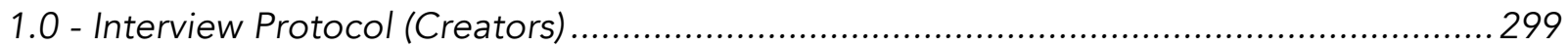

1.1 - Interview Protocol (Majors) ........................................................................................ 303

1.2 Interview Protocol (Policymakers/ Specialists) ....................................................... 307

2.0 List of Web Series Included in Quantitative Diversity Analysis................................... 310

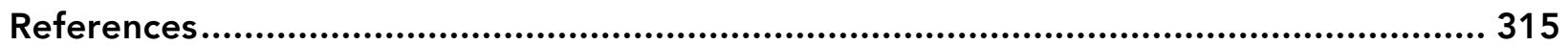




\section{List of Tables}

Table 1 Contextual Dimensions of Television Production \& Distribution

Table 2 Contextualization of Non-Monetary Motives for Entrepreneurial Engagement of

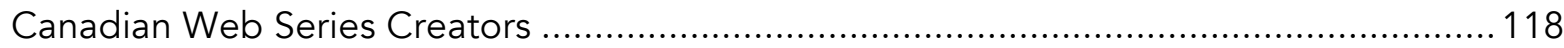

Table 3 TV/ Film Model of Production vs. Web Series Model of Production .......................... 158 


\section{List of Figures}

Figure 1 Gender Representation in IPF Funded Web Series............................................128

Figure 2 Gender Representation in Self-Funded Web Series........................................... 128

Figure 3 Female Representation in Web Series vs. TV (Average) ...................................... 130

Figure 4 Female Representation in Web Series vs. TV \& Film ............................................. 132

Figure 5 Visible Minority Representation in IPF \& Self-Funded Web Series........................... 135

Figure 6 IPF Web Series Representation by Race \& Gender ............................................ 140

Figure 7 Self-Funded Web Series Representation by Race \& Gender ................................. 140

Figure 8 \% of Total Visible Minority Credits by Gender in IPF Web Series: Director ............... 141

Figure $9 \%$ of Total Visible Minority Credits by Gender in IPF Web Series: Writer ................. 141

Figure $10 \%$ of Total Visible Minority Credits by Gender in IPF Web Series: Cinematographer

Figure $11 \%$ of Total Visible Minority Credits by Gender in Self-Funded Web Series: Director

Figure $12 \%$ of Total Visible Minority Credits by Gender in Self-Funded Web Series: Writer.. 143 Figure $13 \%$ of Total Visible Minority Credits by Gender in Self-Funded Web Series:

Cinematographer..... 144

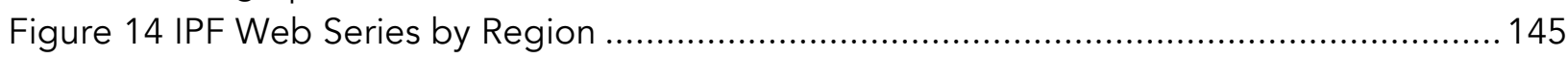

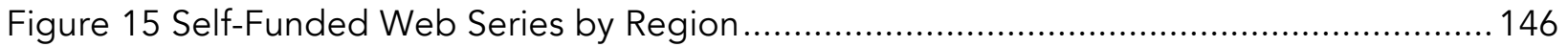

Figure 16 ON Web Series Visible Minority Representation in Key Roles vs. General Regional

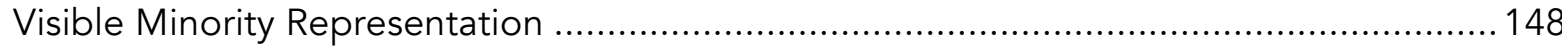

Figure 17 Shared Roles in Writer, Director or Cinematography Categories ......................... 161 


\section{Chapter 1 - Introduction}

Why did I make a web series? Because television stopped giving me money and also put all of their rules onto me. 'You can't put it on YouTube. You have to put it on our website. It's only gonna run for five months. It's gonna be on at this time. It's gonna be on Sundays at 10 o'clock.' Okay...bye... If I'm going to starve, I'm going to starve on my own terms. And there was no...incubation... [A] handful of shows of people in my generation got a shot. And so, this idea that we rejected television, is sort of silly. I think TV rejected us, and we landed on the web...I'm not a snob about the Internet versus television. It's just that TV in this country was so behind the times. It didn't understand that you have to put some stuff on the web. I could go through every network right now in this country and explain to you how they're doing these things badly. (Dan Speerin, Vice President, Independent Web Creators of Canada)

Despite decades of government support and intervention, the Canadian

traditional scripted television sector has failed to produce widespread and consistent

economic and critical success either at home or abroad. Domestically produced

television series hold a small share of the English-language domestic market (CMF,

2016; Coutanche, Davis \& Zboralska, 2015) and account for $16 \%$ of viewing (CMF,

2016). The absence of success in these areas has primarily been attributed to an

unforgiving, too-small domestic marketplace, and unintended, negative externalities

emerging from a complex media policy structure that has been shown to create

disincentives to both product and process innovation (Armstrong, 2010; Berkowitz,

2016; Grant \& Wood, 2004; Picard, Davis, Papandrea \& Park, 2016; Vipond, 2011).

Canadian broadcasters' forays into original programming have been characterized as extending from their regulatory requirement to do so, rather than from genuine and 
deep desires to excel in the art and craft of storytelling, and to push either forward. In addition to this, the Canadian television industry has been shown to suffer from an inequitable organizational structure in which there is an underrepresentation of women and visible minorities ${ }^{1}$ in key creative roles both on and behind the screen (CMG, 2013b; Coutanche \& Davis, 2013; Coutanche, Davis \& Zboralska, 2015; Davis, Shtern, Coutanche, \& Godo, 2014; Kaye \& Davis, 2011; WIFT, 2012; WIV, 2015). Through the assumptions they make, broadcasters act as gatekeepers to both jobs and the stories that get told. To say then that the historical relationship between Canada's major broadcasting incumbents, and the Canadian creators who produce the content that populates (a portion of) their largely, US-dominated airwaves has been fraught with tension, is an understatement of grand proportions.

Until recently, Canadian creators had few options outside of this system of regulated broadcasting incumbents. The availability and uptake of broadband internet at speeds conducive to online video streaming, as well as the routinization of viewing professionally produced television, and 'television-like' content over the web, has opened many possibilities for the Canadian storytelling community. Through 'Web 2.0' platforms (those allowing user interaction and user input), Canadian creators can now access audiences directly without having to obtain anyone's permission. According to

\footnotetext{
${ }_{1}^{1}$ For the purposes of consistency, this dissertation adopts the Statistics Canada (2015) term, "visible minority", to refer to "persons, other than Aboriginal peoples, who are non-Caucasian in race or nonwhite in colour". It is acknowledged, however, that "racialized person" is the more commonly utilized and preferred terminology today.
} 
the media regulator, the Canadian Radio-television and Telecommunications Commission (CRTC), the new broadband-based technologies and services offer Canadian creators an "unprecedented opportunity", and "extraordinary possibilities" and open "doors to niche markets unimaginable even a decade ago" (Blais, 2013).

The realities of utilizing the internet as a distribution channel for online scripted stories ('web series' or 'web-first series or 'digital series'), and their production, however, have been greatly understudied, particularly from a Canadian perspective. Many scholars in their examinations of the online space, have also primarily focussed on the experiences of 'typical', rather than 'professional' users, and have thus tended to foreground the ways the web has generally expanded opportunities for communication and participation (Benkler, 2006; Burgess and Green, 2009; Jenkins, 2006; Shirky, 2008; Tapscott and Williams, 2007). What this research often overlooks, however, are the complex and intricate dynamics undergirding Web 2.0 infrastructure including its political, social, institutional and spatial elements, as well as the power and primacy of economic concerns, which are used to heavily pre-structure online participation and determine its quality and extent. There is also a dearth of research that examines the impacts of this pre-structuring on those who aim to use the web to develop professional scripted screen careers, or the asymmetrical power relationship between Web 2.0 platforms, and the creators who populate them with their work. 
Using a unique, blended approach, this dissertation crucially, and by design, crosses disciplinary boundaries, bringing together a theoretical framework grounded in the critical political economy of communication with critical entrepreneurship studies to get at the issues noted above. This approach pushes forward the boundaries of critical political economy, while answering the call for the contextualization of entrepreneurship studies (Erkko, Kenney, Mustar, Siegel \& Wright, 2014; Watson, 2013; Welter, 2011; Welter \& Gartner, 2016; Welter \& Xheneti,, 2013; Zahra and Wright, 2011; Zahra, Wright \& Abdelgawad, 2014). The novel lens utilized in this dissertation integrates a sustained focus on Canadian creators as entrepreneurial agents with the powerful, big picture, context-setting explanatory capacity of the critical political economy approach, to shed light on creators' motives and the spatial, temporal, institutional and social dynamics that affect their chances of finding success online. The integration of the two different focal lenses uniquely positions the work here to move fluently between structure and agency, and to examine in a more fulsome and meaningful way, how Canadian web-first creators are interpreting, internalizing, resisting and indeed shaping larger institutional and structural dynamics and discourses in and through their practices, organizational efforts and texts.

Canadian creators' forays into online series are viewed here as acts of 'entrepreneuring' through which they aim at emancipation, which involves both breaking free from, and breaking up the status quo (Rindova et al., 2009). Through 
their efforts, they set out to create change for themselves, and sometimes for others, and are motivated by goals that extend past economics alone. This dissertation crucially examines the winding path of their entrepreneurial journeys, and how on the long road toward their goals, they collide with an intricate and intersecting nexus of obstacles emanating from a wide variety of sources, some domestic, and some foreign, and some old, and some new. Although the harsh realities of producing scripted content for distribution over the web often lead to less than ideal economic outcomes, and their accomplishments are rarely as they first envisioned, when looking at Canadian web creators' entrepreneurial efforts through the lens of emancipation, the changes they have been able to achieve, in the absence of many of the traditional industry supports, are not only worthy of notice but ought to be further encouraged by the public sector and industry alike to help move the Canadian scripted screen storytelling system in the direction of one that is more inclusive, innovative, diverse and competitive.

Although their success is neither even nor universal, and in spite of the many challenges they face, Canadian web creators, through their entrepreneuring, have been able to develop a wide breadth of new, tangible, and meaningful storytelling and production skills where opportunities to do so were few. They have attained wellrounded understandings of the production process, and cultivated an intimate knowledge of, and relationship with, their audiences and key demographics. They have 
gained a deeper grasp of the unique resources required to maximize chances of success in the digital-first space, as well as their limitations in these areas. Through their work, they have been able to shine a light on issues meaningful to them, to tell the kinds of stories that have been systemically excluded and to reach communities that have been overlooked by mainstream media.

Canadian web creators have secured gigs, licensed and sold their series globally, won prestigious domestic and international awards, grown and captivated audiences in the millions, succeeded in climbing up in the Canadian storytelling world by winning competitive grants or funding, run successful crowdfunding campaigns based on the brands they have painstakingly grown, and have used their web series as proofs of concept to successfully pitch television studios both here and in the United States.

Not only does the nuanced and complex picture that is uncovered in this dissertation begin to fill important knowledge gaps in the area of independent online scripted storytelling, access and participation, and the importance and role of domestic and international factors and forces, but it also identifies actionable, evidence-based priority areas for policy. The work developed here also builds on and extends Christian's pioneering research in the area of online series (Christian, 2012a; 2012b; $2012 c$; 2014), with findings that suggest that independent web series creation is a global movement in which creators worldwide engage in an effort to challenge existing 
screen industry norms in their own jurisdictions. The findings here therefore critically reaffirm the continued importance of geographical borders to the borderless digital world by demonstrating that the forces and factors that lead creators to engage in web series creation in the first instance, as well as their chances for success, are deeply affected by the practices and structures in place in the jurisdictions in which they are based, and that understanding these specificities is crucial for the formulation of evidence-based, effective policy.

This dissertation also presents an urgent call to action for public sector policy and planning: Without meaningful and swift intervention at this pivotal moment, the digital-first space is likely to replicate and perpetuate the problematic patterns, structures and issues present in the Canadian traditional scripted screen industry, including the continued underrepresentation of women and visible minorities in key creative roles, as well as the loss of creative talent to the United States. In addition, tremendous potential exists to strategically utilize independent web series production as a tool to effect desirable policy outcomes, but such outcomes cannot be achieved through market forces alone.

The dissertation aims to answer the following research questions:

1. What institutional level dimensions and forces, both Canadian and foreign, have shaped and are shaping the Canadian web series industry and its market, and how? 
2. What are the perceived and actual opportunities and challenges of pursuing a web-first strategy for Canadian creators, and what motives do creators have for engaging in this space?

3. Is there more diversity (gender and racial) in the composition of creative teams in the web-first sector than in the traditional television and film sectors? If not, why not?

4. What barriers exist to participation in the scripted digital-first sector, and to finding success (economic and otherwise) in this space?

5. How does producing content for the online space affect the genre, quality and orientation of content that is produced?

6. Which policy priorities and mechanisms are needed to improve Canadian creators' opportunities for success in the online space and to encourage inclusiveness, innovation, and sustainability in Canada's screen storytelling ecosystem?

To get at these questions, the empirical methods utilized in this study are mixed, and multiple. The detail-oriented, micro-level view is vividly and painstakingly unearthed through the course of semi-structured in-depth interviews conducted with 48 individuals active in the production of online scripted content between the fall of 2015 and the summer of 2016. Interviewees were mostly independent creators of scripted online series, including senior executives of established media production firms (i.e. firms that have demonstrated a capacity to produce profits from their media properties for several years or more), founders of independent production company startups, and individuals making online scripted series who are acting on their own. Several interviewees were executives working at legacy broadcasters. Other informants were those who have a specialized understanding of the digital storytelling space 
including funding program executives, and advocates. Most interviews were conducted in-person and lasted between one and a half, and two hours. Several were conducted electronically (through Skype) or over the phone. Most occurred on a one-on-one basis, although several were conducted in groups with creative teams. Interviewees came from a wide cross-section of backgrounds, identities and career stages, and their work spanned a diverse range of genres including those that have been traditionally neglected by mainstream media.

The individuals interviewed here paint a rich picture of their experiences, and through their own words, detail their struggles and successes, and reflect on their place in the larger content ecosystem (both global and domestic) and its structure. While it is quotes from these interviewees that are used to illustrate and punctuate the findings of this dissertation, added richness and analytical depth were achieved through participant observation, which was conducted at industry conferences, award shows and other gatherings over a period of two years, as well as the researcher's own deep embeddedness in the web series community as first, a member of the board of directors of Canada's preeminent non-profit association of web creators, the Independent Web Creators of Canada (the IWCC), and more recently, as its president.

To get at critical questions surrounding diversity and participation in the absence of formal (or obvious) gatekeepers, gender and race were examined across the key creative roles of director, writer, and cinematographer in 175 original seasons 
of independent (i.e. non-broadcaster affiliated) web series produced between 2010 and 2015. To create a point of comparison, series receiving competition-based funding from the Independent Production Fund (IPF), the key fund open to web creators available at the time, as well as series receiving no formal media funding or grants (i.e. "self-funded" series, for the purposes of this study), were examined. This analysis was undertaken with the aim of producing the first benchmark diversity indicators for the digital scripted content sector in Canada, and to compare these results with those in the traditional television space.

Why scripted online content? One of the fundamental elements of the critical political economy approach is its commitment to moral philosophy which favours positions that aim to expand the field of democratic action in all features of social life (Golding and Murdock, 1996; Mosco, 2009; Winseck and Jin, 2011; Wasko, Murdock and Sousa, 2011). A central object of concern for scholars working in this tradition has been the production of news (Haven et al., 2009), and the reason for this is clear-news is, after all, "the oxygen of democracy" (Fletcher and Everett, 2000) without which all hope for a well-functioning society turn asunder. The researcher hopes however, with and through this work, to extend critical political economy's concern for social justice and democracy to the production of scripted drama, which the author believes, plays just as crucial a role in the health and wellbeing of a society as does news. 
Political philosopher Will Kymlicka (1995) argues that having access to one's culture is critical to freedom. His work aims to provide a justification for special minority group rights in polyethnic states, on the basis of liberal principles. Although he does not extend his argument to the domain of the media, the connection is not difficult to see. For Kymlicka (1995) there are two preconditions for the good life-firstly, that we live our lives from the 'inside out', i.e. that we are free to pursue our beliefs and values; and secondly, that we should have the capacity to question these beliefs and values in an intelligent way by being exposed to other viewpoints, and ways of life. Narrative storytelling is critical to the provision of both of these preconditions. It allows communities to speak to themselves-to reflect themselves in ways that are meaningful to them. And it exposes citizens, in perhaps a more vivid and palpable capacity than any other cultural form, to other ways of life, and to other viewpoints and ideas. Scripted narrative audio-visual storytelling makes choices meaningful: through its characters and stories, it can create role models that reify certain paths, making them more attainable. It allows people to see themselves, to see their own follies and virtues reflected back at them in the form of stories on the screen.

The dismissal of scripted content as mere 'entertainment' misses these important points. The full potential of the media to nourish the preconditions of the good life requires that all groups, regardless of gender, race, age, ethnicity, creed, socioeconomic status, physical ability or otherwise, have an equal chance of 
participation in the production of scripted stories, and should be limited by their own talent and ambition alone. This means that as a society, we must work hard to ensure that all citizens have an equitable chance at entering and advancing in the profession. It is important to note here also that while the "constitutional and legal scaffolding" for diversity is stronger in Canada than in many other countries (Murray, 2009), Canadian multiculturalism is an ongoing and complex project that is far from being the utopian "mosaic" it is so often portrayed (Chazan et al., 2011; Zboralska et al., 2017).

Scripted storytelling is also the most resource-intensive form of audiovisual storytelling and requires collaboration and organization between what are normally crews of individuals with specialized skills. This raises questions for democratic access and participation. Can anyone truly make a web series, and who is advantaged in this space?

The dissertation proceeds in the following way: Chapter 2 discusses, in depth, the study's theoretical approach, and provides conceptual justification for the chosen hybrid analytical framework. The chapter begins by situating the current work in the context of media studies more broadly, differentiating its goals and approaches from other streams of research (including cultural studies and production studies), and defending its primary grounding in the critical political economy of communication. The utility of Rindova et al's (2009) 'entrepreneuring as emancipation' perspective is then explained, and the combination of this approach with critical political economy is 
justified. It is shown how the combined approach better captures the dynamic relationship between structure and agency by viewing online creation as deliberate acts aimed at change. The chapter then proceeds with a review of the key literature, and examines the way access to the media in both television and the web has been conceptualized by critical scholars.

The web is then explored through the lens of participatory culture (Jenkins, 2006), and the limits of this view are exposed through critical scholarship in the area which demonstrates that despite the appearance of freedom, online participation is highly determined by corporate platform owners through the ways they prestructure (Butler, 2016; Kelty, 2016; van Dijck, 2013; Wasko and Erickson) the mode of interaction in order to best serve their interests, which often revolve around maximizing profit. YouTube is then specifically examined as a site for participation, and its trajectory of professionalization and institutionalization is also explored (Cunningham, Craig and Silver, 2016; Lobato, 2016; Mann, 2014).

The observation that there appears to be more "cultural progressivity", or the improved participation of traditionally marginalized groups (Cunningham and Craig, 2016) on social media entertainment platforms such as YouTube is also examined. The observation is revisited throughout the dissertation, and its scope is eventually refined through its findings. 
Finally, Cunningham, Craig and Silver's (2016) NoCal vs. SoCal distinction, a key concept applied throughout this dissertation, is introduced. These two different logics, the former born out of Northern California's (NoCal) Silicon Valley and internet pure play companies, values automation, scaling, rapid prototyping, and iteration, while the latter, born out of Southern California (SoCal), values "the core Hollywood skills of acting, screenwriting or directing" (Cunningham, Craig and Silver, 2016, p.10). These distinctions are then applied and extended in a novel way throughout the work undertaken here to help explain why a scripted web series, as a form that typically adopts a SoCal logic, is disadvantaged in several important ways on a platform like YouTube, which rewards and prioritizes NoCal style production.

Chapter 3 then details the study's three empirical methods including semistructured in-depth interviews, quantitative analysis of the digital-first scripted workforce, and participant observation. The chapter justifies the selection of these methodologies and demonstrates how they reinforce the study's goals. It also explores the subjectivity of the researcher, and how her positioning across intersecting identities has motivated and shaped the study's approach and guiding questions.

Following the hybrid framework outlined in the second chapter, Chapter 4 begins by outlining the contextual elements that undergird Canadian creation for the web by examining in detail, and mapping out, its spatial, temporal, institutional and social dimensions through the lens of critical political economy. This important step 
sets up the foundation for an analysis of the research findings, by providing a clear picture of the various constraints that Canadian creators face in their environments.

In the next section of the chapter, the contextual analysis is then combined with the 'entrepreneuring as emancipation' framework (Rindova et al., 2009) and serves as an illuminating lens through which to explore the various motives Canadian web creators have for engaging in digital-first production as revealed through the interviews. The section shows how creators aim to escape the constraints of the industry in which they are situated, and seek out new opportunities to overcome traditional industry gatekeepers to forge alternative pathways to changes they seek. A myriad of motives is revealed, with some aiming to find a way to break into the establishment, and others aiming to create completely new zones for growth outside of the traditional industry spaces in which they feel rejected.

By paying attention to the contextual dimensions undergirding entrepreneuring in this sector, and focusing on the processes of breaking free and breaking up, significant insights are revealed about the 'who' and the 'why' of web creation in Canada. These insights are key to the policy recommendations outlined in Chapter 8 since through them we can adjust the focus of policy so that it better suits, and responds to, who becomes involved and why.

Chapter 5 examines the important relationship between identity and web series participation, and reveals the results of the analysis of gender and race across key roles 
in both web series receiving IPF funding, and in web series not receiving funding from any formal media funding or grant programs. Despite the fact that women and visible minorities are still underrepresented in this space, self-funded web series in which there is no external, formal gatekeeping apparatus, appear to provide important inroads to the participation of these groups in roles in which they are most underrepresented in traditional film and television. The situation is shown to be much worse in more professionalized, funded web series, where the data closely resemble patterns in the traditional media sector.

To contextualize these results, the web series model of production is then outlined and compared to the general model of production present in creative industries, which has been shown to contain systemic disadvantages for women and visible minorities (Eikhof and Warhurst, 2013). Excerpts from the interviews are then used to demonstrate how the web series model of production is actually more intensified than the model present in the creative industries more generally, and how this intensification mitigates some of the freedom that comes from not having a formal gatekeeper, thereby amplifying the importance of social filters like age, gender, race, ethnicity, and class in this space. The chapter therefore reinforces the notion that access does not guarantee capacity, and that diversity should not be taken for granted even on the 'gatekeeperless' web. 
Chapter 6 examines challenges to Canadian content creators that cannot be isolated to Canada's borders. The chapter demonstrates how the shape of the online content distribution ecosystem is being rapidly carved out and determined by a decreasing number of increasingly powerful transnational firms that dictate the terms of creators' participation. Interviews with creators punctuate and illustrate the consequences of this predicament, demonstrating how their livelihoods rest uneasily in the hands of powerful corporations that make substantive changes to their platforms without warning. The chapter also explores how global trends in consumption and attitudes toward the value of online content also affect creators' capacities to make a living from their work, including advertisers' assumptions around the value of online audiences, and a misperception that digital content is somehow automatically more efficient than television content and therefore less deserving of higher production budgets.

The scripted web series form is also shown to disadvantage creators in the global attention economy. The challenges of creating, distributing and sustaining web series that value SoCal time-honoured crafts on platforms that prioritize a NoCal logic are demonstrated, and the 'rock and a hard place' predicament faced by creators is explored. Through reflections on the findings, the chapter also crucially introduces the concept of the Participatory Culture Paradox, or the contradictory set of relations that enables creators' activities in the online space, and at the same time, constrains their 
capacity to find audiences and monetize their work. This concept is key for understanding the uneven effects of Web 2.0 platforms on typical vs. professional users.

Chapter 7 then explores sources of challenges for web creators that emanate from within the Canadian domestic system, and the complicated nexus of tensions and conflicts that arise when institutions, policies and processes erected in the previously established system of production collide with the realities of an increasingly globalized and rapidly moving media world. A problematic picture is revealed in which much power is still held by broadcasters, who, according to the creators interviewed, have generally shown inconsistent commitment to their online ventures and insufficient leadership. The chapter details creators' experiences navigating broadcaster relationships, particularly in situations in which they partner to produce their web series.

The chapter also demonstrates how conflicts of interest within Canadian broadcasters affect the possibility for wider change: individual broadcasting executive informants reveal that despite having a desire for transformation, they are often restricted in their capacity to act by the strength of existing legacy practices, institutionalized hierarchies and processes.

The effects of agreements, conventions and programs that were traditionally designed for television, and extended without much modification to web production 
are also explored, including the potentially troubling consequences of the new media agreement of the key Canadian performers' union, the Alliance of Canadian Television and Radio Artists (ACTRA), as well as the structure of funding programs. Canadian creators' difficulties in capturing the attention of popular streamers like Netflix are also explored, and suggest again that border and place are still important in the new media sphere. The chapter then presents early evidence that suggests that brain drain of Canadian digital talent is likely to emerge due to the various restrictions present in the Canadian digital production ecosystem.

Chapter 8 then examines the outcomes of the entrepreneurial efforts of Canada's web creators through the changes they have been able to enact, despite the existence of the many challenges explored in the proceeding chapters. The changes include new production skills, stronger, well-rounded understandings of the production process, acceleration and amelioration of their careers, as well as the bringing of attention to issues most meaningful to them.

The chapter then delves into policy and program recommendations that aim at encouraging these changes and empowering creators to succeed further, while reflecting on the overall findings of this work. The chapter highlights areas of policy priority that will need to be urgently addressed if the potential of web series is to be harnessed in meaningful and useful ways to encourage inclusivity, innovation and 
competitiveness in the wider Canadian scripted, screen storytelling ecosystem and to overcome its problem areas. 


\section{Chapter 2 - Setting the Stage: Theory and Literature Review \\ Theory}

Determining how, and under what lens to study media industries in ways that meaningfully capture both their macro and micro-level dimensions, and the interaction between these dimensions, has been a challenge for scholars. The desire to recognize the impact of systems and structures, while at the same time make room for, and recognize, the power of human agency is not limited to scholars in the field of communications, but rather, to anyone seeking a fuller understanding of social phenomena. The theoretical paradigm developed here, which is intentionally and necessarily interdisciplinary, multi-method and multifocal, responds to a growing acknowledgement of the need to better address these issues in studies of media industries.

Crucially, the framework recognizes the importance of viewing creators' actions as both affecting, and being affected by, larger structural forces. The approach can best be described as a revised critical political economy (CPE) approach that expands CPE's typical domain of interest and its usual methodologies to better capture the dynamic relationship between structure and agency by viewing online creation through the lens of 'entrepreneuring'. This blended framework's attention to both macro and micro level dimensions brings to the forefront the Participatory Culture Paradox, or the contradictory set of relations that enables creators' activities in the online space, and at 
the same time, constrains their capacity to find audiences and monetize their work. This phenomenon will be explained in detail in Chapter 6, but it is noted here simply to demonstrate the utility of a theoretical approach that incorporates a multilevel analysis.

Why CPE as the Foundation?

CPE is concerned with the way that politics and economics interact to affect the allocation, production, distribution, and consumption of communicative resources. According to $\mathrm{CPE}$, it is the dynamic and mutually constitutive relationship between these two realms, which enables a capitalist economy in the first place. While there are many variants of the CPE approach, each version shares certain central tendencies including an emphasis on de-centering objects of study and examining them in their historical context, looking at issues holistically by taking into account the wider social totality and drawing from many disciplines to derive a deeper understanding, a commitment to moral philosophy which typically involves favouring of positions that aim to expand the field of democratic action in all features of social life, and a devotion to praxis which requires moving beyond contemplation to action (Golding and Murdock, 1996; Mosco, 2009; Winseck, 2011; Wasko et al., 2011). CPE is thus both a theory and a method. It is necessarily non-essentialist, and examines objects in context, recognizing that the things that get made arise out of particular political and economic conditions. CPE crucially acknowledges the interaction between the symbolic and 
economic elements of communication, and that different methods of funding and organizing cultural production "have traceable consequences" for the outcomes of production and how audiences can access them (Golding and Murdock, 1991, p.15).

CPE takes issue with, and spotlights, asymmetrical relations of power between actors in society. It identifies the power of business and capital, and the processes endemic to these, as the starting point for the analysis of social phenomena. It acknowledges that different economic systems will have differing effects on the allocation, production, distribution and consumption of resources, but it does not however, reduce social phenomena to economics alone.

\section{CPE, Structure and Agency}

$\mathrm{CPE}$, through its fundamental ideation, emphasizes examining things in context rather than as discrete objects. This extends to issues of the human mind, and theories of CPE generally recognize that human thought does not arise out of a Cartesian-style dualism that views the mind as completely separate from the external circumstances and conditions of the world in which the thinking individual is embedded. According to CPE, human action is linked to one's place in the world and is shaped by an individual's history, experiences, and economic and social positioning. This does not mean that human activity is determined or in any way 'fated', only that it is shaped by a myriad of shifting and intersecting forces. Contrary to criticisms of CPE dating back to the base- 
superstructure theory of Marx, there is ample conceptual space for focusing on the micro-level practices, preferences and behaviours of individual actors within the broad conceptualization of CPE. And while this is true, CPE has tended, through its typical areas of focus and methodologies, to prioritize and foreground the analysis of structures and institutions over individuals and practices (see for example the work of Babe, 1990; McChesney, 2008; Raboy, 1990; Schiller, 1999; Smythe, 1981). While the progression of thought, and indeed the field of CPE itself, made as a result of these various treatise cannot be emphasized enough, there is much to be learned from the analysis of the micro-level domain, when examined and placed in its wider context.

This dissertation will show, for example, how a greater depth of understanding can be gained from individuals directly about how the increasing consolidation of the web, for instance, has a direct impact on digital creators' chances of success, and moves them to alter their practices in an effort to "game" the system to better these chances and to reduce risk. Examining these practices in context demonstrates how they shape the 'system' in important ways, and together, with an analysis of the contextual dimensions undergirding the practices, reveal important insights about the overall trajectory of the development of the web as a space for video, the emergence of new gatekeepers and the decline and/ or persistence of older ones, as well as the cumulative impact of the confluence of these factors on who participates in online creation, and on the content and form of the media that emerges. 
In recent years, there have been calls within the field of CPE to diversify its main areas of focus, and to more deeply analyze the connection between structure and agency by engaging in midrange and micro-level studies and integrating them into their larger, political and economic contexts (Hardy, 2014; Mosco, 2009; Kellner, 2009; Garnham, 2011). One answer to this challenge comes from a stream of research recently referred to by some scholars as, "Critical Media Industry Studies" (CMIS) (Haven, Lotz and Tinic, 2009). Scholars working within this tradition combine the general area of interest of Production Studies (PS) i.e. the study of the cultural practices of media producers, with an examination of larger institutional level discourses. Scholars working within this tradition ground their work in a Cultural Studies (CS) paradigm. Unlike CPE and its predominant focus on macro-level trends and issues, CS has predominantly engaged in micro-level analyses of audiences (ex. Fiske, 1987), symbol creators (ex. McRobbie, 2004) and texts (ex. Baudrillard, 1983). This highly interdisciplinary approach has emphasized individual agency and its role in shaping culture and society.

There has been a tendency in some CS research to focus on culture, representation and discourse as discrete objects of study, and for this reason the approach has been criticized for being disconnected from the wider context in which cultural products are produced, distributed, and consumed, especially by political economists (see for example Garnham, 1995). There have thus been calls within the 
field to better ground CS in the wider social totality and to connect it to larger critical and normative concerns and frameworks (Kellner, 2001). CMIS is an answer to this criticism. Scholars working in this tradition (see for example, Caldwell, 2009; Christian, 2012; Conor, 2014; Holt \& Perren, 2009; Lotz, 2009; Mayer, 2009; Mayer et al., 2009) emphasize looking at production communities as cultural systems with their "own tacit and explicit, yet contested, rules" (Haven et al., 2009, p.248). They examine, the myriad ways in which specific discourses are constructed and articulated at various institutional sites including policy, regulation, production practices, distribution, and marketing and how such discourses are incorporated or resisted in the practices of cultural workers. (Haven et al., 2009, p.248-249)

The broadened CPE approach developed here can therefore be said to fit under the general CMIS project. Proponents of CMIS argue that,

the way in which institutional discourses are internalized and acted upon by cultural workers is an important missing link between political economy's concentration on larger economic structural forces and much of cultural studies' analyses of end products such as media texts and audience interpretations. (Haven et al., 2009, p.247).

Work in this area therefore seeks to expose how cultural workers internalize, resist and affect these larger institutional dynamics. While the broadened CPE approach that is developed here (and will soon be discussed) can therefore be said to fit under the general CMIS banner, its goals are different than those typically found in work from this emerging field. First, the approach developed here has a strong commitment to praxis, which is foundational to CPE; it aims to offer recommendations for policymakers and 
other stakeholders based on its findings. Second, it is, at its core, aimed at facilitating and ameliorating issues of social justice, as is also inherent to CPE. While there is certainly room for these considerations and commitments in the broad project of CMIS, work falling under the banner often has not delved deeply into either of these dimensions.

One of the most promising starting points that extends the CPE perspective into new dimensions is Mosco's (2009) conceptualization which foregrounds processes rather than entities. Mosco (2009) identifies three processes that form the foundation of his version of CPE: commodification, or the process of transforming use value into exchange value; spatialization, or the process of utilizing communication technologies, for example, to diminish the constraints of physical space in the wide domain of social life; and structuration, or the process by which, "structures are constituted out of human agency, even as they provide the very 'medium' of that constitution" (p.17). Although the work developed here will demonstrate the effects of commodification and spatialization on the professional world of Canadian digital-first creators, and will critically reflect on, and probe, the larger reasons for these trends, it prioritizes the understanding of structuration which is the important domain in which these forces come together and create both opportunities and constraints for action. A focus on structuration will therefore reveal the important ways in which structure and 
individual agency collide, and will thus demonstrate new spaces of potential for policy intervention.

According to Mosco (2009), a political economy approach to structuration foregrounds and critically examines the power relations between agency and structure. For Mosco (2009), "society and the individual create one another" and so, "we are the product of structures that our social action or agency produces" (p.185). Importantly, as Mosco (2009) and others make clear, because of the fundamental conceptualization of political economy, and its core theoretical constructs and focus on the social totality, a considered analysis of agency is not possible without an analysis of structure, because structure is the platform from which human actions emanate.

To put it another way,

Structuration theory is an approach to social life that aims to address goaloriented, reflexive human action, without giving up on understanding the 'sutures' of power that mutually constitute social action. (Mosco, 2009, p.186)

Mosco (2009) contrasts this understanding with that of Giddens (1984), the originator of both the term, and of a particular conceptualization of the structuration process. Mosco (2009) argues that from the standpoint of political economy, Giddens' (1984) conceptualization is limited in several important ways: first, it lacks a focus on power relations, the kind of focus that is central to a CPE approach; second, it is missing a critical approach to social life, and is disconnected from matters of morality; and third, it tends to prioritize individual agency, due to its particular conceptualization of 
structure. Unlike Giddens' (1984) general theory of social life, a political economy approach to structuration would provide an account of how structuration operates under a particular set of historical circumstances and conditions (Mosco, 2009). While political economy typically involves analyzing the macro-dynamics of power, a CPE emphasis on structuration will require examining how power operates at both the bigpicture, structural level, alongside "the constitutive, interactive, or micro-level" (Mosco, 2009 , p.187). This kind of examination requires different tools and methodologies than CPE tends to employ which means moving beyond the analysis of "data about revenues, organizational structure, employment, as well as submissions to government bodies" (Mosco, 2009, p.187). For Mosco (2009) then,

[S]tructuration is an entry point to examine the mutual constitution of structure and agency in political economy. It is a starting point for expanding the conception of power and, in addition, it provides a lever for understanding the forms that social relations take in political economy. (p.188)

In addition, a meaningful commitment to structuration requires an approach that examines the role of social factors such as gender, race, age, sexuality and class (in the relational, rather than categorical sense) in the organization of power, agency and structure (Mosco, 2009). These factors are central to grasping how relationships of power operate, since they provide foundations for the organization of resistance to power and give rise to social movements. As such, a fulsome CPE account of structuration must make clear how an individual's capacity for meaningful action is both 
constrained by structures and social factors, and how these structures and social factors serve as the foundations for action, resistance and change.

While the promise of a CPE approach to structuration is strong, there is no firm roadmap of its application to creative work. Although work from the CMIS approach (see for example Caldwell, 2009; Conor, 2014; Holt \& Perren, 2009; Lotz, 2009; Mayer, 2009; Mayer et al., 2009) provides a vital starting point, it generally does not devote sufficient attention to the central concerns of CPE as outlined above.

\section{A Contextualized Entrepreneurship Studies ${ }^{2}$}

While CPE is criticized as being too focussed on macro-level, structural dimensions—entrepreneurship studies has been criticized for taking the opposite approach. Scholars working within entrepreneurship studies are calling for a substantive re-evaluation of its assumptions and methods, and take issue with its focus on 'the entrepreneur' as central figure and discrete object of study (Johannisson, 2011; Watson, 2013; Welter, 2011; Zahra and Wright, 2011). These scholars argue that studies of entrepreneurial actors and their activities are best understood in context and insist that a more holistic approach to entrepreneurship studies is required.

Watson (2013) argues that a shift away from studying the entrepreneur as discrete object is necessary for a "full appreciation of how entrepreneurship works"

\footnotetext{
2 Some of the work presented here was published in: Zboralska, E. (2017). No more status quo! Canadian web-series creators' entrepreneurial motives through a contextualized "Entrepreneuring As Emancipation" framework. International Journal on Media Management, 19(1), 29-53.
} 
(p.411). For him and others (Erkko, Kenney, Mustar, Siegel \& Wright, 2014; Watson, 2013; Welter, 2011; Welter \& Gartner, 2016; Welter \& Xheneti,, 2013; Zahra and Wright, 2011; Zahra, Wright \& Abdelgawad, 2014), 'contextualizing' entrepreneurship research is key to gaining a more complete understanding of entrepreneurial activity, including motives and outcomes, and to formulating more accurate and meaningful theory. According to Zahra et al. (2014), an understanding of context forces a more intimate relationship to emerge between the researcher and the phenomena under investigation. Indeed, instead of being "reporters of distant events and issues, researchers are expected to become engrossed in the dynamics that shape context" (Zahra et al., 2014, p.494). This yields a fuller understanding of the topic at hand. By examining the various dimensions of context, researchers are able to "map out" the multitude of micro-processes involved in entrepreneurial activity to gain a more multifaceted grasp of how it is that they work (Zahra et al., 2014, p.481). Taken together, these various contextual dimensions "provide raw material from which entrepreneurial actions spring" (Zahra et al., 2014, p.481). As Welter (2011) crucially observes, understanding contextual dimensions and their interactions is key "...for understanding when, how, and why entrepreneurship happens and who becomes involved" (p.166), or as Zahra et al., (2014) put it, contextual influences "pervade and influence the micro processes that give entrepreneurial actions their substance and potency" (p.480). They undergird all entrepreneurial behaviour. 
In order to facilitate the integration of contextual understanding into entrepreneurial research, scholars have put forward classifications of contextual dimensions key to entrepreneurial activity. Such classifications aim at providing researchers with more robust tools to be able to explain, and more fully appreciate the complex sets of interactions involved in entrepreneurial activity. Zahra and Wright (2011) synthesize these classifications into four chief contextual dimensions. The first is the spatial context, which denotes the physical and geographical environment; the second context is the temporal context which includes historical dimensions and their influences and trajectories; the third is the social context which includes the wide set of human interactions in the entrepreneur's world including professional networks, family and friends; the fourth and final context is the institutional context which includes the rules, regulations and norms that establish the rules of engagement.

According to Welter and Xheneti (2013), the institutional, social and spatial dimensions constitute the 'where' of entrepreneurship, while the temporal dimension constitutes the 'when'. Importantly, contexts are not stable in the sense that their boundaries are constantly shifting (Zahra and Wright, 2011). These dimensions, taken together, along with an understanding of their dynamism, provide the explanatory power necessary to answer the 'why' and 'who' of entrepreneurial activity (Welter, 2011). Indeed, the 'who' is not a context in and of itself, but rather "reflects the impact of contexts on entrepreneurship" (Welter, 2011, p.167). A contextual understanding 
can thus reveal more about why certain people choose to engage in entrepreneurial activity. Scholars further insist that to have explanatory power, studies incorporating context must be able to integrate the multidirectional interaction between contextual dimensions, and between the entrepreneur and these dimensions Watson, 2013;

Welter, 2011; Zahra \& Wright, 2011; Zahra et al., 2014). Interactions are mutually constitutive, and recursive; the entrepreneur affects these contextual dimensions just as much as the contextual dimensions affect her. Indeed, as Spedale and Watson (2014) point out, they are part of "one big system" (p.762). The problem is, however, few entrepreneurship studies to date have integrated contextual analysis in meaningful ways, and in ways that accommodate and acknowledge the important two-way relationship between an entrepreneur and her context (Spedale \& Watson, 2014; Welter, 2011; Zahra et al., 2014).

What does a 'contextualized theory of entrepreneurship' look like? A synthesis of the literature on contextualization and entrepreneurship studies reveals a 'wish list' consisting of three core elements. Based on this synthesis, contextualized theories of entrepreneurship should:

1) present a deeply-rooted application of contextual dimensions that moves past examining them as discrete variables (Spedale \& Watson, 2014; Welter, 2011). According to Welter (2011) this is the 'lens' view of context, which involves "a broad perspective, drawing attention to who, what, when, where, and why" (p.167). 
2) adopt a process-oriented perspective that can make sense of the multifaceted interactions between contextual dimensions, and between the entrepreneur and these dimensions (Johannisson, 2011; Spedale \& Watson, 2014; Welter, 2011; Zahra et al., 2014).

3) widen the scope of what typically counts as entrepreneurship, and avoid confining entrepreneurial activity to the economic domain (Johannisson, 2011; Watson, 2013; Welter, 2011; Zahra and Wright, 2011).

Although it has never been explicitly connected by its authors to the call for a contextualized entrepreneurship studies, Rindova et al.'s (2009) entrepreneuring as emancipation perspective implicitly contains, by virtue of its main focus and constructs, the core foundational elements to support a contextualized theory of entrepreneurship. Firstly, Rindova et al. (2009) adopt the term 'entrepreneuring' to signify a widening of the scope of what is typically classified as entrepreneurial activity in mainstream theories of 'entrepreneurship' (Rindova et al., 2009). According to the view, entrepreneuring encompasses "efforts to bring about new economic, social, institutional, and cultural environments through the actions of an individual or group of individuals" (Rindova et al., 2009, p.477). These efforts are fundamentally linked to a desire for change, and the view thereby does not restrict entrepreneurial activity to the economic domain. Viewing entrepreneurial activity in this broader sense responds to studies, which demonstrate that many times, wealth creation is not the sole, or even 
main goal of entrepreneurial actors (Rindova et al., 2009). This is particularly true in the case of the creative industries wherein individuals have been shown to hold a wide variety of motives outside of economic benefit, including first and foremost, a desire for autonomy (Hesmondhalgh and Baker, 2011), their "love" of the work (Gill, 2011), viewing their activity as a "labour of love" (Friedson, 1990), an answer to a fundamental "calling" which they cannot help but answer (Kris \& Kurz, 1987), and a variety of "social, political or cultural mission[s]"(Hoag and Compaine, 2006).

For Rindova et al. (2009), there is too much at stake in merely examining business strategies, for example. These strategies, according to the authors, are often grounded in deep-seated desires for change, which cannot be ignored.

Secondly, the entrepreneuring as emancipation perspective is activity-oriented and moves away from examining entrepreneurship through entities, turning the focus instead onto processes. The perspective seeks to examine entrepreneurial emergence by envisioning entrepreneuring as fundamentally comprised of two key processes: 'breaking free' and/ or 'breaking up' (Rindova et al., 2009, p.480). The former entails a desire for autonomy, and freedom from constraint, whereas the latter entails a desire to disrupt, and create change. The authors argue that one or both of these two distinct but related aspects of emancipation may be present in an entrepreneurial act, and both are often already implicitly co-present in studies and theorizing about entrepreneurial activities. The reinterpretation of the founding story of Google through 
the entrepreneurship as emancipation lens demonstrates that although Google's success has been linked to the "superiority of their PageRank technology, the entrepreneuring process began with a dream to break free from perceived technological constraints (in terms of the available algorithms for conducting search) and cultural constraints (in terms of the information content that these algorithms made available)" (Rindova et al., 2009, p.481). The breaking up aspect of their activity involved "downloading the internet to improve upon early search engines" (Rindova et al., 2009, p.481). By examining entrepreneuring as aimed at change creation, the perspective allows a deep understanding of the relationship between constraint and entrepreneurial activity to emerge.

According to the authors, the 'breaking up' aspects of emancipation are closely linked to the Schumpetarian view of 'entrepreneurship as creative destruction', but the emancipation perspective moves beyond this view by also examining the 'breaking free' component. Breaking up becomes not the "means" of entrepreneurship, as is the case in the Schumpetarian view, but rather, "one of its goals" (Rindova et al., 2009, p.481). As the authors make clear, "entrepreneuring involves creating and amplifying cracks in otherwise stable (and potentially rigidified) social and economic relationships that impose constraints on certain types of activities that the entrepreneur him/herself and other members of their social world may value" (Rindova et al., 2009, p.481). 
Entrepreneuring as emancipation thus weighs heavily in its analysis "the factors that cause individuals to seek to disrupt the status quo and change their position in the social order in which they are embedded-and, on occasion, the social order itself" (Rindova et al., 2009, p.478). A fulsome understanding of entrepreneurial emergence from this paradigm therefore requires a deep grasp of the conditions that an entrepreneurial actor is attempting to break free from (ex. limits, structures, accepted norms, patterns, processes etc.), as well as an understanding of the dynamics that she would like to break up through her attempts to create anew (ex. changing an established system by breaking into it; ameliorating her position in an existing hierarchy; creating an alternative system etc.). According to Rindova et al. (2009), the constraints from which individuals seek emancipation may be "of an intellectual, psychological, economic, social, institutional, or cultural nature" (Rindova et al., 2009, p.479). Moving into the language of contexts, grasping these constraints implies a deep comprehension of the various contextual dimensions (spatial, temporal, social and institutional) as synthesized by Zahra and Wright (2011), as well as a movement away from the study of these contexts as discrete objects.

CPE and Entrepreneurship Studies: An Unlikely Marriage

Rindova et al.'s (2009) framework, in combination with a deep focus on contextual dimensions, is the ideal prism through which to analyze the entrepreneurial 
emergence in the scripted, Canadian digital-first sector. What is missing from this framework, however, is a larger, overarching view of contextual dimensions, their interactions, and how they both have an impact on, and are affected by individual agency. CPE has much to offer entrepreneurship studies by way of its holistic approach, and grounding in the wider social totality. It is a sharp tool for the analysis of macro-level, structural issues and processes, and when applied to the process of structuration, as suggested by Mosco (2009), it reveals deep insights about how agency and structural dynamics interact. CPE's commitment to moral philosophy also provides entrepreneurship studies with a much needed grounding aimed at democratizing access to entrepreneurial tools and resources, and increasing the likelihood of success of Canadian entrepreneurial actors regardless of challenges related to identity, including age, gender, race, class or sexuality. The contextualization of entrepreneurship studies through a CPE approach provides the theoretical engine and glue through which a deeper understanding can be derived by placing the issue of power at the centre. The 'entrepreneuring as emancipation' framework's focus on the agent-centric desire for change fits well with CPE's interest in the organization of social movements. As will be shown later in this work, Canadian creators' motives for engaging in entrepreneurial activity are part of movement to disrupt a certain set of power relations and conditions that constrain their opportunities for success. 
Entrepreneurship studies also has much to offer CPE. To start, because the entrepreneuring as emancipation framework examines entrepreneurial activity as fundamentally aimed at change, the chief question, "change from what?" becomes of central importance, providing CPE a vital entry point into the analysis of agency against the backdrop of structure. CPE's commitment to praxis is also reinvigorated by viewing creative work through ideas from entrepreneurship studies. Thus far, CPE has largely ignored the social phenomenon of entrepreneurship. Governments are increasingly interested in policy to promote entrepreneurship, particularly in the context of the creative sector. Adopting a view toward entrepreneurship allows CPE to 'speak the language' of policymakers and business, all the while retaining the theoretical framework and conceptual constructs required to critically evaluate the issues, and to make meaningful recommendations. Combining CPE with entrepreneurship studies also acknowledges the current realities of our time, while in the same instance, never losing sight of the fact that the current framework is not inevitable. We live in a capitalist system in which non-commodified spaces are limited; while it is important to continue to encourage the expansion of non-commodified public spaces, for instance, it is also important that CPE can begin to envision other 'alternatives' to how 'alternative media' has often been conceived under this paradigm. This moves CPE away from what has sometimes been perceived as disconnected, 'pie-in-the-sky' thinking, and crucially acknowledges that there are important ways in which our 
commercial media system can be made to work better (more inclusively, more openly, more transparently) without the all-or-nothing, non-commercial or bust, mentality that has sometimes been advocated. The tools of CPE can, and indeed should, be used to rightfully point out the limits of entrepreneurial action in the face of systems and structures, to dispel myths of the entrepreneurial process, to add depth and context to the understanding of entrepreneurial motives, and to demonstrate the dynamic relations of power involved in entrepreneurial activity.

\section{Review of the Literature}

Critical Political Economy of the Traditional Television System

As a mechanism for the exchange of information, the traditional television broadcasting system enabled a one-way flow of information, and contained distinct roles for the 'sender' and 'receiver'. The sender was prioritized in this chain of flows, and communicative power rested primarily with her. In media terms, individuals were divided into 'producers' and 'audiences', respectively, and there was no crossover between these roles. The formal capacity for audiences to respond to broadcast content did not offer any degree of nuance: audiences could express their approval or disapproval of broadcast content by clicking on or off a program or channel.

Critical political economists of the media have traced problems along the pipeline of this tightly contained and managed system. Access to participation in the 
media, or more specifically, who gets to tell the stories (and who doesn't), and whose stories are represented (and whose are not) are central concerns for political economists. The most radical of these positions views television as a tool and 'missionary' of capitalism, and a vehicle for the representation of dominant private and government interests, as well as a method to suppress alternative perspectives and dissent (Herman \& Chomsky, 2008; Herman \& McChesney). This position sees television as an instrument of capitalism and both a 'buyer' and 'seller' of its ideology. While other critical political economists take issue with the strong instrumentalist nature of this view (see Winseck \& Jin, 2011), all political economists recognize problems in the way that the quest for profit affects the kind of content that is produced, and who gets to produce it. Scholars point out that because the television system operates according to a dual product logic whereby content is produced for both audiences and advertisers-the content that emerges from this system becomes skewed toward only the most desired (lucrative) demographics (Croteau and Hoynes, 2001; Meehan, 2006). This filtering process has led to the limited representation of alternative viewpoints, struggles and stories (McChesney, 2008; Winseck 2002, 2008; Arsenault and Castells, 2008; Croteau and Hoynes, 2001; Kunz, 2007; Skinner and Gasher, 2005; Cooper, 2005; Shade, 2005). High barriers to entry in traditional television also made it difficult for content containing alternative viewpoints to be produced and disseminated. 
Critical political economists have also argued that the high levels of consolidation in the mass media system present problems for democracy and further limit the diversity of voices and perspectives within the system. Due to the highly unpredictable "nobody knows" market for media products (Caves, 2000), and the high sunk costs related to product and infrastructure development, and increased competition from other information and entertainment sources, scholars have argued that media industries tend heavily toward oligopoly or monopoly (McChesney, 2008; Almiron, 2010; Arsenault and Castells, 2008; Croteau and Hoynes; 2001). Already large media corporations then aim to grow even larger in an attempt to be able to withstand losses, and to acquire the competition in the ongoing battle for audience attention (Croteau and Hoynes, 2001). These large, consolidated corporations then create significant barriers to entry for potential competitors (Skinner and Gasher, 2005). Television is often just one asset among many, and these firms typically own other media assets including radio and print, and the 'pipes' to transmit this information including cable and, more recently, internet and mobile. Television producers had to rely on this highly complex pipeline to get their content distributed and exhibited.

The Internet, 'Participation' and its Spaces and Players

Enter the internet. The web, and the digitization of information, provides a fundamentally different underlying mechanism for the exchange of information than 
does traditional television. In direct opposition to the one-way flow from sender to receiver, or producer to audience-the open web enables multi-directional, one-tomany communication. The distinction between 'producer' and 'audience' becomes increasingly blurred in the online space, as individuals can respond to, upload, and interact with, content in ways never possible before. Some scholars (Benkler, 2006; Burgess and Green, 2009; Jenkins, 2006; Shirky, 2008; Tapscott and Williams, 2007) focus their analyses of this new space on its apparent discontinuities from the system of past. These scholars tend to foreground the democratic aspects of the web-viewing it as a space that has greatly expanded opportunities for meaningful communication and access to the media and systems of distribution, above all else. In Jenkins' (2006) own words, in the new online space— "every important story gets told..." (p.3). According to the scholars who tend to foreground the democratic capacity of Web 2.0, the various new web platforms and services have given rise to a 'participatory culture' movement, which stands in stark opposition to the more passive form of media viewership that comes with traditional television distribution:

Rather than talking about media producers and consumers as occupying separate roles, we might now see them as participants who interact with each other according to a new set of rules... (Jenkins, 2006, p.3).

According to Jenkins (2006), this participatory turn is part of the larger era of convergence, which is characterized by "...the flow of content across multiple media platforms, the cooperation between multiple media industries, and the migratory 
behaviour of media audiences who will go almost anywhere in search of the kind of entertainment experiences they want" (p.2). This process, according to Jenkins (2006), represents more than just a technological shift, but involves a deeper "cultural shift as consumers are encouraged to seek out new information and make connections among dispersed media content" (p.3).

Jenkins (2006) argues that in the era of convergence, the role of consumers is amplified—-they do not merely accept convergence, but play a significant role "actually in driving the process" (Jenkins, 2006, p.8). For them, "[t]he promises of this new media environment raise expectations of a freer flow of ideas and content" and inspire consumers to "bring the flow of media more fully under their control and to interact with other consumers" (p.18). According to Jenkins (2006), 'interaction' is not the same as 'participation'. Interactivity, for him, merely signals designed environments that increase opportunities for consumer feedback. Participation is different, he argues. In contrast to interactive environments where "what you can do is...prestructured by the designer", participatory environments are those which are "more open-ended, less under the control of media producers, and more under the control of consumers" (Jenkins, 2006, p.133). His work focuses on the ways that the web has permitted the development of "...many unauthorized and unanticipated ways of relating to media content" (p.133)—spaces where 'true participation' (and not merely interaction) is possible. 
Although research about the professional production and distribution of scripted, web-first content is limited—a growing body of literature is emerging about one of the most significant platforms for the distribution of this content: YouTube (see Andrejevic, 2009; Bird, 2011; Burgess and Green, 2009; Cunningham, Craig \& Silver, 2016; Ding et al., 2011; Gardner and Lehnert, 2016; Morreale, 2014; Van Dijck, 2013; Vonderau, 2016; Rodriguez, 2016; Salvato, 2009; Strangelove, 2010).

YouTube's now famous (or infamous) slogan, "broadcast yourself", proclaims that for it, participation is a core value. According to the company, "YouTube allows billions of people to discover, watch and share originally-created videos" and "provides a forum for people to connect, inform, and inspire others across the globe..." (YouTube, 2016). Indeed, creators can post videos to YouTube without formal permission, agreements or sales. The popular press has tended to glorify the YouTube platform, often spotlighting its rags-to-riches successes, and focusing on its emancipatory qualities, and apparent role in the democratization of distribution and media more generally, without critically examining its business practices or the lived realities of creators negotiating the platform (see for example Barnard, 2013; Brownell, 2014; Kane, 2014; Oliviera, 2012).

For Burgess and Green (2009), who examine YouTube as primarily a cultural system, the platform appears to be an equalizer in that it is a hybrid space where 
"amateur and entrepreneurial uses... are not separate, but coexistent and coevolving" (p.103). According to them,

[C]ontent is circulated and used in YouTube without much regard to its source - it is valued and engaged with in specific ways according to its genre and its uses within the website as well as its relevance to the everyday lives of other users, rather than according to whether or not it was uploaded by a Hollywood studio, a web TV company, or an amateur videoblogger. (Burgess and Green, 2009, p.54).

Although Burgess and Green (2009) are somewhat careful not to overstate the newness of YouTube, arguing that it does not represent a "radical...break" with history, but rather, a period of "increased turbulence, becoming visible as various established practices, influences, and ideas compete with emerging ones as part of the long history of culture, media, and society" (Burgess and Green, 2009, p.14)—they, like Jenkins (2006), ultimately do not pay enough attention to the economic system undergirding the platform and Web 2.0 more generally, or the various other complex dynamics at work including important political, social, institutional and spatial elements. While the web has certainly enabled new avenues for participation in the media for ordinary users, critical scholars examining these other elements question the quality and extent of this participation on both YouTube and the web more generally (see Andrejevic, 2009; Bird, 2011; Butler, 2016; Couldry, 2011; Fuchs, 2014; Gillespie, 2010; Kelty, 2013; Mosco, 2004; Muller, 2009; Verstraete, 2011; Van Dijck, 2013; Vonderau, 2009, 2016; Schiller, 2000). 
Scholars (Butler, 2016; Kelty, 2013; van Dijck, 2013; Wasko and Erickson) point out that contrary to Jenkins' (2006) interaction vs. participation distinctionparticipation on the web is always limited and prestructured by the platform owner and in important ways is not open-ended. Others argue that contrary to Burgess and Green's (2009) and Jenkins' (2006) assertions regarding the relative equality of content on Web 2.0 platforms-professionally produced, higher-production quality content, associated with established traditional production studios and programs, captures more of YouTube users' attention, and is increasingly privileged in this space (Rodriguez, 2016; Fuchs, 2014; Morreale, 2014).

Van Dijck (2013) summarizes the continuities between YouTube and traditional media well, arguing that despite the fact that the platform does not control content on YouTube in the traditional sense i.e. by picking and choosing, this does not mean that "its content flows unmediated" (p.113). Instead, she argues, it is heavily steered by search engines and ranking algorithms. YouTube's interface design and its underlying algorithms select and filter content, guiding users in finding and watching certain videos out of the millions of uploads, for instance through buttons for "most popular" videos. The site controls video traffic not by means of programming schedules but by means of an information management system that steers user navigation and selects content to promote. Even though users feel they have control over which content to watch, their choices are heavily directed by referral systems, search functions, and ranking mechanisms...(Van Dijck, 2013, p.113)

The undergirding system architecture therefore has real and significant consequences not only on what content users can view, but also on the chances of success of the 
content that is uploaded. Indeed, as scholars have also shown, the notion of ubiquitous participation on YouTube, and Web 2.0 platforms is largely a myth (Butler, 2016; Ding et al., 2011; Fuchs, 2014). Ding et al. (2011), for example, found that just four percent of YouTube users provide nearly three quarters of the content.

Another stream of research has emerged on the professionalization of YouTube as a platform for content creation and its increasing maturation as an economic system (Cunningham, Craig and Silver, 2016; Lobato, 2016; Mann, 2014). This research examines YouTube's journey from its radical beginnings as a rebellious alternative to television (in both values and content) to its current status as a Google-owned institution. As Lobato (2016) writes, "[t]he site has changed profoundly, shedding the youthful exuberance of its early years - the 'broadcast yourself' era - and morphing into a more structurally complex, managed ecosystem designed to monetize both amateur and professional content" (p.348). This growing up has been accompanied by new initiatives by YouTube to encourage the further professionalization of its content creators including new 'YouTube Spaces' that act as hubs for training, collaboration and provide infrastructure to creators with 10000 or more subscribers (including one in Toronto) (YouTube, 2016b), the 'Creator Academy', which includes YouTube's own educational materials aimed at the creator community (YouTube, 2016c), as well as the administration and development of the backend for 'Multichannel Networks' also known as MCNs. There has been increasing scholarly interest in YouTube's coming of 
age, especially with the recent rise of MCNs, since they represent the introduction of an intermediary into a system, which largely began as a horizontal zone (see the work of Cunningham, Craig and Silver, 2016; Gardner and Lenhert, 2016; Lobato, 2016; Vonderau, 2016).

Briefly, MCNs are intermediaries that provide "technical, promotional and advertising services in exchange for a commission" (Lobato, 2016, p.351). They function similarly to Hollywood agents, but their role extends past mere representation. They represent groups of creators who are typically unified by an area of interest or the way they cater to a similar demographic, and house these creators under a single umbrella, increasing opportunities for audience discovery and cross-pollination.

According to those examining MCNs in depth (Cunningham, Craig and Silver, 2016; Gardner and Lenhert, 2016; Lobato, 2016; Vonderau, 2016), MCNs reintroduce scarcity into a system that is over-saturated with the "structural oversupply of video content" in order to "regain control over pricing and contribute to establishing a cultural repertoire across which risks can be spread" (Vonderau, 2016, p.367). It is clear that MCNs do not benefit all users equally (Vonderau, 2016) and their utility and ethics of their practices have been called into question (Gardner and Lehnert, 2016). As Lobato (2016) points out, MCNs are apt to go after creators with content that more easily translates to commercialization and consumer markets, including "beauty, cooking, gamer and fashion videos" (p.358). Lobato (2016) thus theorizes "that 
producers working in these verticals may professionalize at a faster rate than in other parts of YouTube, driven in part by the resources made available by MCNs" leading to "differentiated effects across the YouTube landscape" (p.357-358). He argues that while there is now growing recognition of the increasing commercialization of the YouTube platform, we must now "scrutinize specific logics of commercialization at work within particular parts of that ecosystem, on the understanding that these are not monolithic in their operation or effects" (Lobato, 2016, p.349).

Production of Scripted, Serialized Content for the Web

There is a dearth of scholarly work that focuses on web-first production through the lens of particular streams of content (ex. vlogs vs. scripted web series), even though this deeper focus is more likely to bring to surface the unevenness of using the web as a platform for distribution, and the complex set of economic, social, institutional, cultural and spatial dynamics that affect the performance and shape of the content. Such disaggregated, focussed study is of extreme importance, especially when the goal of the work is to inform policy, as is the case here.

Scholar A.J. Christian has been at the forefront of the inquiry into the production of scripted, serialized content for the web. He (2012a; 2012b; 2012c; 2014) explores why and how independent American creators opted to produce scripted series for the web in what he identifies as the medium's peak years, between 2006 and 2010 . He 
discovers "skilled but disempowered workers" (2012a, p.29) who strove to reinvent the "forms of production, storytelling, marketing and distribution" associated with traditional television (p.2). His findings demonstrate that web series creators often took to the web after feeling marginalized by the mainstream industry in some way (Christian, 2012a). This marginalization stemmed either from identity characteristics such as gender, or race, or area of interest, which was often deemed too niche, or too outsider, to be considered a viable market by mainstream television (Christian, 2012a). According to Christian (2012a; 2012b; 2012c; 2014), the web presented a platform for these individuals to take action and respond to the stratified Hollywood market-a space that they perceived to be unwelcoming to their stories and labour.

Christian (2012a) theorizes that post-2010, scripted web series content "would achieve 'scale" ' and "capture the attention of advertisers who would otherwise market on television" (p.203). He points to the large investments in the hundreds of millions of dollars made by Netflix and Google into original programming by 2012, and the cash infusions into independent web distribution platforms including My Damn Channel and Blip TV (now defunct). The implication appears to be that post-2010, web-based scripted content market would grow exponentially more challenging (particularly for independent creators), and significantly more institutionalized. Putting this into Christian's (2012a) own terminology, "off the line" spaces (i.e. "spaces in which 
industry workers try to forge a...market for independent creators") (p.2) would be subsumed by both Hollywood, and Silicon Valley, and thereby be brought on the line.

The question is, what happens post-2010, for independent creators and firms who wish to find success online but have less capital and power than Hollywood and Google?

Similarly to Christian (2012a), Cunningham and Silver (2013), theorize that the digital marketplace is likely to enter a stage of maturity, with "an emerging oligopoly of dominant firms whose profitability may also begin to stabilize" (location 155).

According to the authors, these new firms are likely to be Silicon Valley giants, rather than current Hollywood incumbents, since they appear to be "remaking the criteria for future TV networking" through their global aspirations, infrastructure and scale (Cunningham and Silver, 2013, location 1107).

These companies, according to the authors, are poised to dominate the future of online entertainment, since they are

Internet 'pure-play' companies that already had or have been able to develop a critical mass of online customers and possess extensive data on their past online search behaviour and purchasing habits. In addition, they have years of experience marketing directly to their customer base targeting those most likely to be interested in a particular genre or program based on web analytics of each individual's past behaviour and any product feedback that they may have provided. (Cunningham, Craig and Silver, 2016, p.4)

What unifies these firms, according to the authors, is an underlying logic, which is fundamentally different from non pure-play internet companies like the Hollywood 
studios (Cunningham, Craig and Silver, 2016). This logic is rooted in their historical origins as 'NoCal' companies born out of Northern California's Silicon Valley. Their logic stands in contrast to Hollywood's 'SoCal' (Southern California) logic. YouTube, in its Google era, can be seen, according to the authors, as "a history of Google seeking to come to terms with the SoCal fundamentals of entertainment, and content and talent development, from its NoCal base as an IT company dedicated to scale, automation, permanent beta, rapid prototyping and iteration" (Cunningham, Craig and Silver, 2016, p.4).

This has created both "continuities and contestations with traditional media models" with results that "have been decidedly mixed" for creators (Cunningham, Craig and Silver, 2016, p.4). The authors (Cunningham, Craig and Silver, 2016) argue that SoCal Hollywood firms place value on "time-honoured mass media and premium content strategies" (p.12) and different skills including "the core Hollywood skills of acting, screenwriting or directing" (p.10).

Google's YouTube, according to the authors (Cunningham and Craig, 2016), is characterized by a certain kind of cultural production they call "communitainment", which, reflects the dynamics of a protoindustry facilitated by networked communications technologies (social media platforms), primary strategies of communication as much as content (intense interactivity), more discursive and communicative content genres and formats (most notably, vlogging), and driven by an ethos of community 
(an ecology where fans, subscribers, and supporters directly constitute the communities that trigger the sustainability of content creator careers)...(p.5413)

This kind of production is common to social media entertainment, including Facebook, SnapChat and Twitch (Cunningham and Craig, 2016). Despite the fact that the authors acknowledge the problematic "structural conditions_huge, globe-spanning online platforms whose leaders are far bigger than the Hollywood majors that have had a dominating influence on global media; a fast-advancing algorithmic culture; and the precariousness of online labor", they also observe that there is increased "cultural progressivity" on these platforms "to a larger extent...than in older, established global media orders" (p.5413-5414). This progressivity, according to the authors, entails "a rapidly professionalizing and monetizing wave of diverse, multicultural, previously amateur content creators from around the world" (p.5412).

The authors argue further that the kind of "hegemonic domination of distribution and IP-controlled content informed by media scarcity, highly capitalized ownership, and captured regulatory regimes" differs greatly from platforms like YouTube which "feature content abundance and near-unlimited access—conditions that generate agency and affordances...and offer greater cultural and language diversity" (Cunningham and Craig, 2016, p.5414).

While agreeing that creators do indeed have more access to distribution platforms than they did previously, the dissertation will delineate the limits of this 
access, and will emphasize the effects of the highly prestructured YouTube architecture on the kind of content that gets made and its chances of success. The YouTube ecosystem as a distribution platform, and its various opaque matrices and modes of commodification, is highly complex, and as Lobato (2016) points out, requires scrutiny as to the unevenness of its effects. Work in this dissertation will demonstrate how and why creators with primarily SoCal sensibilities (i.e. those who produce scripted, serialized content) have been particularly disadvantaged on YouTube as a platform, and how its inherent architecture creates specific challenges for their chances of success, from both an audience reach and economic standpoint.

The analysis of the workforce behind the Canadian, scripted, serialized web-first content created from 2010-2015 (most of which was released on YouTube), narrows the scope of Cunningham and Craig's (2016) observation regarding the increased presence of diversity in the social media entertainment universe. The work here thus emphasizes the importance of examining unique web content streams separately, as each particular form carries with it its own inherent modes and logics of production and engagement. The dissertation will also demonstrate why more narrow examinations are also particularly useful in the context of policy. This is especially true in Canada where the government appears eager to embrace the optimistic, feel-good framing that has been associated with the free-market strategies of the internet, in lieu of regulation-a 
decision that may, in the end, prove to be problematic for the Canadian content creation industry, and its long-term survival, inclusivity and capacity to innovate.

YouTube itself appears to be increasingly adopting more SoCal practices: it recently launched YouTube Red, a subscription service which features not only usergenerated content, but importantly, also original YouTube IP. Importantly, the market for advertising on YouTube is also "becoming less and less viable as a sustaining revenue base for both creatives and intermediaries like MCNs" due to a severe over saturation of content leading to the decreasing value of individual advertising impressions (Cunningham, Craig and Silver, 2016, p.6).

By utilizing the expanded CPE approach detailed in this chapter, this dissertation will be able to capture the way structure and agency interact in the market for web-first content, and how creators of scripted, digital-first series navigate this complex environment. The study will provide additional depth to the examination of this particular stream of production, revealing important insights that can be utilized to inform policy. Christian's (2012a; 2012b; 2012c; 2014) work in this area primarily focuses on what he calls the "peak" period for independent, scripted web series. The work here picks up where Christian leaves off, examining what happens 'post-peak' in the years from 2010 until present.

While Cunningham, Craig and Silver (Cunningham and Craig, 2016; 2017; Cunningham, Craig and Silver, 2016) are also invested and actively working in this 
space through their pioneering conceptualization of the various logics present in the social media entertainment world, their work has just begun to examine disaggregated web content streams (Cunningham and Silver, 2017). Thus, while they now distinguish web series from more "native-to-online...content types" which include "vlogging, gameplay and do-it-yourself (DIY) style and beauty" (p.72), their focus, thus far, has been on providing an in-depth examination of the features that characterize the latter.

Canadian Production of Scripted, Serialized Content for the Web

Finally, the work here will also tell a side of the story that has thus far been largely unexplored —-this is the Canadian side of the story, one that is rich in idiosyncrasies and depth. Production for the web has been an understudied area for Canadian critical scholars. There appears to be no critical scholarly work that explores this nascent market or community, or the experiences of Canadian English-language web creators in the context of an increasingly globalized and consolidated online media space. Two industry led studies have thus far been commissioned by the dominant producers' association, the Canadian Media Producers Association (CMPA), and the recently established online web creators' association, the Independent Web Creators of Canada (IWCC), respectively. The more systematic of the two studies (Nordicity, 2014), examines the Ontario market only, and does not distinguish between scripted web series, and unscripted web shows such as vlogs. The case for examining 
web-based content through a more granular approach, however, has already been established above. The work undertaken here thus aims to begin to fill in this important knowledge gap. The second study (Duopoly, 2015), foregrounds primarily economic concerns, without exploring other significant, non-economic issues in depth.

As both studies demonstrate, there are few domestic buyers for Canadian original online content (Duopoly, 2015; Nordicity, 2014), and top-tier transnational over-the-top firms like Netflix have primarily partnered with established American studios for the biggest-budget, premium online content (Duopoly, 2015). Duopoly (2015) also observes the emergence of a web drama 'class system' in which independently produced, low-budget, and often shorter-form online series coexist and compete with TV-look-alike premium longer-form content, the kind of content that in the terminology of Cunningham, Craig and Silver (2016) most adheres to SoCal Hollywood values.

Nordicity (2014) observes that Canadian web creators are "risk-takers and innovators" who, despite a lack of support infrastructure, and inadequate financing sources, have achieved critical acclaim, winning prestigious international and domestic awards with the content they have managed to produce (Nordicity, 2014, p.37). These creators have been shown to be passionate multitaskers "handling all aspects of business and content" (Nordicity, 2014, p.6), with their main source of financing being personal savings (Nordicity, 2014). This stands in stark opposition to the traditional 
television and film sectors, where $41 \%$ of total financing comes from public sources (a combination of federal and provincial tax credits, subsidies and other funds) (CMPA, 2016). This number is even higher when financing from the public-private Canadian Media Fund (CMF) is taken into account (CMPA, 2016). The entrepreneurial aspect of Canadian web creators' activities and their motives for entering the online space despite the lack of adequate financing sources, stable business models, skills and support infrastructure (Duopoly, 2015; Nordicity, 2014) has yet to be explored. The work conducted here thus aims to fill in this gap by examining the experiences, activities and motives of Canadian creators through a critical lens, and against the myriad of intersecting contextual dimensions that undergird their work. From a policy perspective, understanding creators' activities in the context of what change these creators seek, and what constraints they are working to overcome, as the framing of the entrepreneuring as emancipation approach requires, will reveal important information about the kinds of policy alternatives and priorities that must be embraced in order to empower these creators to achieve the success they envision. Viewing their actions in the context of change also reveals much about the status and health of the wider screen system of which they are a part. The expanded CPE approach developed here will thus reinforce the continued importance of context and territory in the borderless internet video world. 


\section{Chapter 3 - Methodology}

The methodology employed in this study is based in the expanded CPE approach described in the previous chapter. In order to capture both the macro- and micro-level dimensions of the issues, and to more robustly encapsulate the relationship between structure and agency, the study employs a mixed method design that incorporates both qualitative and quantitative components and provides insights at multiple levels. The study's three main empirical methodologies include semistructured in-depth interviews, quantitative analysis of the digital-first scripted workforce, and participant observation. The in-depth interviews conducted here focus on the micro-level dimensions of the issues, as does the participant observation component; the quantitative analysis of the digital-first scripted workforce provides a mid-level view. The findings from these various sources are then placed in context, by examining them against their wider spatial, temporal, institutional, and social elements, as is more typical of CPE approaches. Following a discussion of the study's key methods, the subjectivity of the researcher, and her positioning across intersections of identity are also addressed, and help provide a justification for, and explanation of, the study's particular orientation and framing. 


\section{Qualitative, Semi-Structured In-depth Interviews}

\section{I.a. The Method}

In-depth interviews are one of the most versatile research methods (Cook, 2008) and reveal deeper information than can be derived through other research methods alone (Cook, 2008; Goodman, 2001). Interviewees are assumed to hold "deep understandings" of everyday activities, events or places by virtue of their participation in them (Johnson, 2001, p. 104). By conducting in-depth interviews, the researcher seeks "to uncover what is usually hidden from ordinary view or reflection" so that she may "penetrate to more reflective understandings of the nature of that experience" (Johnson, 2001, p.106).

Since one of the central goals of this study is to capture the process of structuration by examining how agency and structure interact along various key dimensions, it is crucial, as Mosco (2009) observes, for it to move beyond the usual CPE methodologies which center on gathering information "about revenues, organizational structure, employment, as well as submissions to government bodies" (p.187). In-depth interviews are thus an ideal methodology for this purpose and provide the rich, lived experiences of the individuals who are affected by and affect these larger structural elements. They have also been the method of choice of contemporary critical studies of media industries that aim at capturing micro-processes 
and dynamics (see the work of Curtin, Holt and Sanson, 2014; Caldwell, 2009; Mayer, 2011; Christian, 2012; Tinic, 2005; Gitlin, 1983).

The in-depth interviews that were conducted for this study were semi-structured in nature. Semi-structured interviews permit the researcher to maintain "some control over the direction and content to be discussed, yet participants are free to elaborate or take the interview in new but related directions" (Cook, 2008, p.423).

\section{I.b. Sampling}

The selection of participants for in-depth interviews is of vital consequence to the quality of insights revealed, thus "extra care" must be taken to make certain that participants selected for the interviews fit the study's parameters and objectives (Kolb, 2008; p.145). Thus, because in-depth interviews require participants to have specific qualities, sampling is typically purposive and particular to each study, and since it is an intensive methodology, in-depth interviews involve a smaller sampling of participants than is typical of other, more extensive methods, such as surveys (Goodman, 2001; Kolb, 2008). For this study, semi-structured in-depth interviews lasting between one and half and two hours were conducted with 48 individuals active in the production of online scripted content between the fall of 2015 and the summer of 2016. Interviewees were mostly independent creators of scripted online series, including senior executives of established television production firms (i.e. firms that 
have demonstrated a capacity to produce profits from their media properties for several years or more), founders of independent production company startups and individuals making online scripted series who are acting on their own, as well as executives working at legacy broadcasters. Other informants were those who have a specialized understanding of the digital storytelling space including funding program executives, and advocates. Most interviews were conducted in-person, although several were conducted electronically (through Skype) or over the phone.

For recruitment, purposive snowball sampling was used to ensure a wide range of perspectives were incorporated into the sample, including participation from those at diverse career stages (emerging, mid-level and established), backgrounds and production genres (ex. science fiction; youth; comedy; drama; action). A key aim for the researcher was also to ensure participation from traditionally marginalized groups including women, visible minorities, and those who identify as non-heterosexual. To achieve this goal, she would ask participants to personally identify and connect her to individuals who fit these categories. The researcher did not consider participation from these groups to be adequate until data saturation was attained, and repetition could be observed in the kinds of experiences being reported. Of the participants interviewed, $27 \%$ were women, and $15 \%$ were visible minorities; these numbers map onto the levels of representation in the scripted web-first sector, as revealed in the study's workforce analysis, which will be discussed in a later chapter. 
Participants were asked about their professional histories, ex-post reasons for engaging in the web space, their experiences of, and views about, the production and distribution of scripted series for the web, as well as their perspectives on the way their identities, and social and cultural attributes mediate their chances of success, and how these factors might be related to their decision to engage in the web space in the first instance. The interview protocol is attached in Appendix A. The actual names of interview participants are generally used throughout the research findings (with their consent); occupational titles are used for those who wished for their identities to be protected, or in cases in which the author believes protecting the participants' identities is preferable given the sensitivity of matters discussed.

\section{I.c. Coding}

The researcher employed qualitative content analysis methodology to analyze the interview data. Content analysis provides an excellent means of systematically making sense and meaning of data, and of capturing key insights and concepts (Flick, 2014; Given, 2008; Krippendorff, 1980). The goal of content analysis is "to identify consistent patterns and relationships between variables or themes" (Given, 2008, p.120). The interviews were fully transcribed and coded for shared themes, both manifest and latent, using NVivo 11 qualitative data analysis software. As is often the case with the thematic analysis of semi-structured interviews, certain themes were 
expected given that they were explicitly explored through the interview questions and thus acted as starting points for coding categories (Given, 2008). The majority of the coding categories, however, were derived through an inductive process, which is particularly useful in underexplored or new areas of research such as this (Elo \& Kyngas, 2008; Marks \& Yardley, 2004).

Prior to commencing coding, the researcher conducted a deep close reading of the transcripts to gain an appreciation of the breadth and depth of the contents. Categories were refined throughout the coding process. The researcher employed the concept of 'stability' as a measure of reliability of the categories, which has long been a standard of measurement in qualitative content analysis research (Krippendorff, 1980). Stability of categories is attained when the same coder attains the same results by doing another coding pass (Krippendorff, 1980). To ensure reliability then, the researcher conducted two full passes of the data to ensure stability of emergent codes.

As Marks and Yardley (2004) make clear, precise quantitative measurement of codes and themes from small samples can be misleading; instead, as is suggested, when helpful, the researcher provides an indication of the incidence of themes using descriptive qualitative terminology (ex. "some"; "few"; "rarely"). Additionally, if a shared theme or issue was observed unanimously throughout the sample, the researcher indicates this in the description of the findings. Patterns emerging in interview subgroups (emerging vs. established creators, for example) are also noted 
using qualitative frequency indicators, however, quantitative descriptions are provided in exceptional cases that warrant such description. Indeed, the goal of the coding process and thematic analysis was to "stay true to the raw data, and its meaning within a particular context of thoughts, rather than attaching too much importance to the frequency of codes which have been abstracted from their context" (Marks \& Yardley, 2004, p.67). The researcher thus provides both qualitative and quantitative indications of thematic frequency based on the need, applicability and usefulness of such details.

\section{Quantitative Gender and Diversity Analysis of Key Roles in Web Series}

\section{II.a. The Method}

An analysis of gender and racial representation in key creative roles in web series produced between 2010 and 2015 was conducted. To provide richness and depth to the study, the researcher examined two categories of web series: those produced with funding from an institutionalized media funding program, and those produced without such support, herein referred to as "self-funded web series". The reason for the inclusion of these separate categories of web series is to probe whether the funding program apparatus acts as a filtration mechanism, leaving out certain groups of producers. The fund that was chosen for examination was the Independent Production Fund (IPF) as it was, until recently, the sole national fund geared at standalone scripted web series made without interactive elements or broadcaster 
affiliation. While IPF web series do not require a broadcaster affiliate to trigger funding, some nevertheless had such agreements in place. For web series produced without funding from a formal media funding or grant program, another excluding criterion was broadcaster affiliation. The self-funded web series thus are also "independent" in this way. The decision not to conduct a wider examination of scripted web series produced with or by a broadcaster was purposively made because the statistics for the representation of diversity in creative teams in broadcaster affiliated Canadian traditional television content are now known (see WIV, 2015; WIV, 2014; WIV, 2013; WIV, 2012 ).Undertaking an even wider analysis of representation to include broadcaster affiliated web series would therefore have been an inefficient use of the researcher's resources.

To be included in the evaluation in either category, web series had to meet the following criteria:

1. Be in English, scripted, narrative and live-action

2. Be comprised of, at minimum, three episodes

3. Be distributed first over the internet

4. Be distributed over the internet between 2010 and 2015

5. Be aimed at youth or adult audiences (i.e. no content targeted to pre-schoolaged children) 
Gender and visible minority status were examined across producer, director, writer and cinematographer roles. ${ }^{3}$ The foregrounding of these roles is consistent with prior examinations of gender and diversity in screen based industries (see for example Bunche, 2014; WIV, 2014). The researcher also took note of the province of origin of the series under investigation. This allowed for a more meaningful examination of representation levels of visible minority status across the aforementioned categories when compared with regional rather than solely national labour market availability. Initial year of distribution, YouTube view counts of first and last episode, and genre were also recorded, as were notes on the thematic subject matter of the series.

\section{II.b. Sampling \\ Independent Production Fund Web Series}

The IPF produces annual reports revealing series awarded funding in each given year. Because of this, the IPF reports provided a clear, bounded population from which to draw. Web series that did not meet the inclusion criteria noted above were excluded from the study (i.e. those that were French-language, aimed at pre-school-aged children, or were animated). This left a total of 36 individual seasons of series for

\footnotetext{
${ }^{3}$ Only those receiving a "Director of Photography" or "Cinematographer" credit were recorded for the analysis. This means that in cases in which credits included "camera" and "Director of Photography"/ "Cinematographer", only the latter were taken into account. These credits denote that an individual's role is the head of the camera department. For series where there were simply "camera" credits, all names were taken into account in the analysis since there appears to be no traditional hierarchical arrangement.
} 
evaluation. No sampling was thus required and all of the series meeting the aforementioned criteria were included in the analysis.

\section{Self-Funded Web Series}

As this is the first systematic analysis of independently produced English-language web series across Canada, no single, comprehensive inventory of Canadian web series exists. It appears that no formal bodies keep aggregated and up-to-date lists of such series. Since these series have not received formal funding, there are no standardized annual reports from which to draw. Identifying the population was thus a significant challenge in and of itself, and the researcher devoted substantial time to this goal.

The researcher used the following resources to amass the population of web series that fit the outlined parameters:

1) A substantial but incomplete inventory of Canadian web series obtained from the not-for-profit creators' association, the IWCC.

2) Google, YouTube and Vimeo searches preformed using key words.

3) Searches of industry subscription trades magazines (Playback and Cartt.ca).

4) Searches of social media platforms including Facebook and Twitter, including groups aimed at Canadian web creators.

A total of 139 individual seasons of web series distributed between 2010 and 2015 (and fitting with the inclusion criteria) were identified. These were series that remained online and available for viewing. The researcher could not include series that she could 
not find, thus, while the list appears to be comprehensive, it does not include series without a digital footprint that may have been taken down by their owners for any number of reasons.

\section{III.c Data Gathering Procedures}

The researcher gathered data for this component of the study in three steps:

1) First she identified the population of web series to be examined (for both funded and self-funded series)

2) After the identification of the population, she amassed crew lists for each series, recording the names of each individual working in the roles of interest mentioned above.

3) As she recorded the names of key crew members, the researcher would also record the gender and visible minority status of each individual working in these roles.

Each step of the data gathering process was significantly time intensive. Because there is no standardization in the online space, every web series does not, for example, include a credit sequence at the end of the series. The researcher went to considerable effort to amass the names of all members involved in the productions in the roles of interest. This required, at times, sourcing contacts for producers and asking them to provide credits lists and finding the information by tracking down other official texts 
including web sites, official series posters and other data. The researcher crossreferenced the different data sources to ensure accuracy and completion.

Gender and visible minority status were confirmed at the same time. The researcher would conduct an internet search for each name on the crew list. The researcher prioritized official sources to confirm both of these variables, drawing on images primarily from personal Facebook, Linkedln, and other web pages owned by the crew members. In cases where a crew member had a common name, the researcher ensured the accuracy of the images by cross-referencing with other data gathered from trade publications, and journalistic interviews. In cases in which a crew member would have a common name, but did not appear in wider search results, the researcher would ensure accuracy by confirming that the individual in question was linked to at least two other members of the production team on their social media platforms (Facebook or Linkedln).

The determination of visible minority status was more complex-and the challenge of making such determinations in the absence of self-identification has been noted by WIV (2015), an ongoing study that previously examined both gender and race in the traditional television workforce, but opted to only examine gender in its latest release given the challenges.

Following the accepted Statistics Canada (2015) departmental standard, visible minority status was defined as "persons, other than Aboriginal peoples, who are non- 
Caucasian in race or non-white in colour". This category includes individuals who are Chinese, South Asian, black, Filipino, Latin American, Southeast Asian, Arab, West Asian, Korean, Japanese, or a mix of these categories. To make visible minority determinations, the researcher coded anyone who appeared visibly non-white as a visible minority. In ambiguous cases, for instance, in cases of a Hispanic surname, the researcher went to great lengths to determine whether the individual was of Latin American or Spanish (European) descent. This included investigation of familial ties, again through analysis of personal social media networks. There is a possibility that individuals of mixed race who appear white, and have Western or European surnames were miscoded as non-visible minorities.

Despite the rigour with which the researcher carried out this component of the study, given the various overall methodological limitations, the results should be viewed as indicators of participation, rather than definitive, final statistics.

\section{Participant Observation}

Participant observation provides an excellent entry point into understanding a way of life from an insider's viewpoint (Bloor and Wood, 2006; Jorgensen, 1989; Crang and Cook, 2007). It has been described as "deep hanging out" (Wogan, 2004), and its aim is to "uncover, make accessible, and reveal the meanings (realities) people use to make sense out of their daily lives" (Jorgensen, 1989, p.14). This methodology has 
been successfully used in classic studies of production (see Rosten, 1941;

Powdermaker, 1950), as well as more recently (see Christian, 2012; Hesmondhalgh and Baker, 2011; Mayer, 2011; Caldwell, 2008).

Participant observation is particularly useful when the group being studied is "somehow obscured from the view of outsiders", and when "little is known about the phenomenon" under investigation (Jorgensen, 1989, p.12). In the case of this study, the web series industry in Canada is a nascent market about which little is known. Creators participating in this space are members of the traditional television production community, but are also outside of it. These professional circles of practice are often difficult to penetrate without access to the right kinds of networks, and a familiarity with the overall industry structure and an understanding of its operation and functioning.

Familiarity with the phenomena under investigation, including past work in the community under study, or current membership, can provide important advantages when engaging in participant observation (O'Reilly, 2009; Crang and Cook, 2007; Bloor and Wood, 2006). A researcher who is delving into completely unfamiliar terrain might not know enough "to ask the right questions" (Bloor and Wood, 2006, p.73). The researcher has several years of professional practice in the Canadian television industry working in various roles including casting, research, development and production coordinating. She has pitched major production companies and has been involved in well-funded, and low-budget productions. The researcher also remains involved in 
professional practice, and along with three partners, has started a production company that has several projects in various stages of development, including a documentary series aimed at international, over-the-top distribution. This ongoing engagement in professional practice in the Canadian screen industry proved to be immensely useful in building rapport and 'speaking the language' of the study's participants, which amplified the power of the participant observation methodology. The researcher was able to use her understanding of the traditional television industry to more meaningfully engage in the web series community for whom, as will become clear in the findings of the research, the television industry is a clear point of reference.

Participant observation represents a "continuum of activities stretching from pure observation with no participation, to full participation" (Payne and Payne, 2004, p.167). O'Reilly (2009) stresses the fundamental difference between participants and participant observers:

"A participant is simply a member, joining in, gaining access to some interesting discussions, sharing experiences, and witnessing some fascinating and some mundane events... The participant observer, on the other hand, is participating in order to observe, notice, record, and try to make sense of actions and events. This involves an element of standing back intellectually and reflecting on things...". (p.151-152) 
The researcher's deep participation in the web series community began in April 2015 when she volunteered to be an Associate Producer for the second annual "TO WebFest", Canada's largest festival devoted to recognizing accomplishments in Canadian and international web series. The researcher was engaged in this capacity from April to June of 2015. The researcher was, at the outset, open with the festival's organizers about her interest in the web series industry as an object of study. She made clear however, that her motivation for volunteering, and indeed for this study, stems from a deep seated commitment to the success of the Canadian screen sector, which is made clear by her ongoing engagement in the industry and her various policy and advocacy pursuits. During the three days of the festival, the researcher was in charge of coordinating media volunteers, and assisting in field production on the ground. As part of her participation, the researcher gained access to the festival's industry panels which provided her with insider knowledge about Canadian web series creators' overarching concerns, and their inside perspectives on what they believe to be the key issues facing this nascent industry at the individual, independent production firm, and broadcaster levels. Research notes were recorded on an ongoing basis throughout this process. In July 2015, the researcher decided to run for a position on the board of directors of the IWCC, the not-for-profit, parent organization of T.O. Webfest, based on a shared commitment to building a sustainable and inclusive web creator community and industry marketplace. She was appointed to the board, and assumed a 
role as head of the policy committee where she led the organization's participation and efforts in CRTC, and other industry and governmental inquiries, including the recent 2016 Ministry of Heritage consultation on Canadian culture. The researcher has, as of July 2016, been appointed as president of the organization. Although her research is separate from her role as member of the board of directors, her deep engagement and participation in the community facilitated access to research participants for the study. Throughout the years since commencing her work with the IWCC, the researcher has attended and/or organized numerous conferences, industry events, information sessions, panels and informal social gatherings around the topic of web-first content. Her ground-level, first-hand experience of the issues, and the opportunity to work closely and interact with so many key members of the community has pushed forward her conceptual understanding of this nascent area in deep and consequential ways.

\section{Subjectivity of the Researcher}

Although the research undertaken here is objective in its analysis, it is important to discuss, in a project that investigates systemic barriers faced by traditionally marginalized groups, how the researcher's own subjectivity helped shape the project, its framing, and its research questions.

The researcher's status as a woman, an immigrant, and as someone from working class, humble economic means, combined with her professional experiences in 
the Canadian television industry, motivated her interest in the project's area of inquiry and informed its areas of focus.

As an immigrant to Canada with no social, familial or professional links to the creative industries (in Canada or otherwise), the researcher, as a young person striving for a career in such an uncertain, and non-traditional sector, faced many challenges in the initial, 'breaking in' phase. She worked as an unpaid intern for several months at a Toronto production company that specializes in reality television. On top of the 40 (unpaid) hours per week this required, in order to sustain herself financially, she worked an additional 20-30 hours per week in the restaurant service industry. She sustained this pace of work for nearly a year, even after being promoted to a paid position at the company due to the prohibitive costs associated with living in Canada's key production centre. The researcher thus experienced firsthand, the inequitable nature of access to the television industry, and how challenging it is to break in without familial economic support, or existing industry connections. To compensate, the researcher often sacrificed her personal health, family and social life to advance in her career.

The researcher's experience in the reality subsector of the Canadian television industry also sparked her curiosity around the underlying political economy of the production of television in Canada. She became interested in the dearth of scripted production, and observed what she perceived to be a lack of innovation in the kind of 
content being produced. These observations led to an increasing desire to critically examine the business side of television in a sustained way.

The researcher also recalls a particular moment when, while working as a freelance casting researcher on a reality series, an executive at a major broadcaster told the casting team that the participants recruited were "too diverse". This gave rise to a deep interest in the role and power of editorial gatekeepers in the media.

As an individual that spent much of her formative years in a lower income neighbourhood in Toronto, the researcher was surrounded by other newcomers, who were often people of colour. While her family's economic situation was stabilizing, the researcher experienced and grew up with others in similar social and economic circumstances, and witnessed through her relationships and associations, how race, gender, social class and immigration status presented barriers to upward mobility. Together, these experiences, and identities have coalesced to inform her area of interest and her project's sustained focus on equity, inclusion and the removal of systemic barriers.

Thus again, while the research undertaken here is objective in the sense that the researcher is not willingly imposing any artificial limits on the scope and nature of the findings, the fundamental orientation of the project and its areas of focus come from the researcher's past experiences and her positioning across intersections of identity in the ways described here. 


\section{Chapter 4 - The World in Which They Create: The Spatial, Temporal, Institutional and Social Dimensions of Screen Production and Distribution in Canada}

The work here aims at uncovering the process of structuration by analyzing the mutually constitutive relationship between Canadian web creators, and the structures that permeate their world by combining critical political economy with the entrepreneuring as emancipation perspective. The entrepreneuring as emancipation perspective views entrepreneurial agents and their behaviour as fundamentally aimed at change. As was made clear in Chapter 2, this requires a deep understanding of the various constraints that entrepreneuring agents seek to overcome or break up.

Through a synthesis of the critical political economy literature on the institutional, temporal, spatial, and social dimensions of the Canadian screen industry, as well as an analysis of relevant policy in the area-this section provides the reader with an understanding of the world that Canadian web creators inhabit, and the myriad of structures that form the foundation of this world.

Because CPE is a holistic methodology, these various contextual dimensions are examined first in concert. Headings indicate to the reader the various interaction points among these dimensions, although this initial synthesis is meant to be a discursive one. Following this, the various dimensions are then mapped out and visually represented in chart form. Although these contextual dimensions are difficult to capture in stable categories given their mutually constitutive and recursive relationships, when treated as 
"analytical devices" rather than "empirical realities" they can yield insights into the microfoundations of the entrepreneuring process (Spedale \& Watson, 2014, p.762) since they "pervade and influence the micro processes that give entrepreneurial actions their substance and potency" (Zahra et al., 2014, p.480), and are key to "...understanding when, how, and why entrepreneurship happens and who becomes involved" (Welter, 2011, p.166). The goal for this section is to provide a starting point to the question, "what do Canadian web creators seek change from?". Understanding the structures and dimensions, which together form the foundation of their professional worlds, provides a strong base for analyzing how creators choose to use their agency to enact (or attempt to enact) change through their entrepreneurial activities. This will in turn provide a solid ground for generating meaningful insights geared at ameliorating policy in this area.

Institutional

The Canadian communication sector is federally protected, and legislation prohibits the foreign ownership of broadcasting and telecommunications assets. Traditional television in Canada is highly regulated, and governed by the federal Broadcasting Act of 1991 (the "Broadcasting Act" or "the Act"), which contains both cultural and economic goals for broadcasting. The Act explicitly declares that the broadcasting system is "essential to the maintenance and enhancement of national identity and cultural sovereignty" (Part 1, s. 3.1.b) and that through its employment and 
programming, it should "serve the needs and interests, and reflect the circumstances and aspirations, of Canadian men, women and children, including equal rights, the linguistic duality and multicultural and multiracial nature of Canadian society and the special place of aboriginal peoples within that society". (Broadcasting Act, s.3.1.d.iii). The broadcasting system should also "serve to safeguard, enrich and strengthen the cultural, political, social and economic fabric of Canada" (Part 1, s. 3.1.d.i). Traditional broadcasters and distributors are required by the CRTC to achieve these policy goals through a variety of instruments including licensing, reporting requirements about financial, regulatory and equity and diversity issues, scheduling requirements, quotas, and financial contributions, among others. Although online programming services such as Netflix are considered to fall under the remit of the Act according to the Commission, they are, however, exempt from these regulations (Public Notice CRTC 1999-197) for a variety of CRTC-stated reasons, including most recently, that imposing regulations on online services is "not necessary to achieve the broadcasting policy objectives set out in the Act" (CRTC 2015-86).

Due to the restrictions on foreign ownership in communications, the Canadian communications market is controlled by a handful of companies. The level of consolidation in the Canadian system is unprecedented and has been shown to be the highest of all G7 countries (Analysis Group Inc., 2012). On the distribution level, vertically integrated companies hold $81.4 \%$ of the market share (Analysis Group Inc., 
2012, p. 4). Until recently, then, Canadian producers had few alternative partners and distribution options than those supplied by the small number of vertically and horizontally integrated private incumbents (Bell, Rogers, Shaw, Corus and Quebecor), who sell cable, satellite, Internet and mobile services, and own a variety of other media assets including cable and over-the-air channels, newspapers and magazines, or the country's national public broadcaster, the Canadian Broadcasting Corporation (CBC). From here on, the incumbents (including the public broadcaster) will be referred to in the collective as the (Canadian) "majors".

To secure space for independent voices, the Broadcasting Act dictates that the Canadian television system must "include a significant contribution" from independent production companies (i.e. those not affiliated with the majors through ownership) (3.1.h.v); this has traditionally been interpreted through policy by requiring $75 \%$ of all Canadian-produced content broadcast by the majors to have been produced by independent production companies (CRTC 2008-4). Prior to the proliferation of broadband internet and online video services, self-distribution of serialized programming was not an option. Not only were the majors gatekeepers to the distribution of content shown in Canada, they too had, and continue to have, primary control over access to institutionalized funding.

The main funding source for Canadian original television series, the Canadian Media Fund (CMF), which is funded by both the government and required 
contributions from revenues generated by the majors' cable, satellite and IPTV arms, requires what is colloquially known as a broadcast 'trigger licence', or an agreement from a Canadian broadcaster to licence a program for a certain term, to broadcast it in primetime viewing hours, and to commit to it a certain minimum threshold of funds (CMF, 2015). This has created an asymmetrical power relationship between the majors, and the independent production companies and creators that depend on them, for distribution and access to further capital. There is little tradition of venture capital or non-broadcaster private investment in the content industry in Canada. Venture capitalists and other private investors prefer technology-based opportunities, which provide greater chances for high growth, scalability and return on investment (Duopoly, 2016). If the majors do not provide the trigger, securing other financing is challenging. Prior to 2015 , there was only one national funding source, the private Independent Production Fund (IPF), which was open to creators of standalone scripted web series without the need for an agreement from one of the majors. Two new national public funding opportunities have emerged since 2015, through Telefilm and the CMF respectively, although their scope is smaller, and the funding available is significantly less than that which is available to traditional television made in partnership with the majors. The details surrounding access to these new funds, and their implications for the nascent web-first industry, will be discussed in further detail in chapters 7 and 8 . 
Spatial and Institutional

Scholars have characterized the way Canadian majors (excluding the national broadcaster, given its particular cultural mandate) generally treat Canadian original programming, as a burden that must be endured in exchange for the regulatory protections that are received, rather than as an opportunity (Berkowitz, 2016; Grant \& Wood, 2004; Picard, Davis, Papandrea, \& Park, 2016). The economics of the Anglophone Canadian screen industry do not work in favour of original production. The Canadian Anglophone television market is small, consisting of approximately 32 million people, especially in comparison to its same-language contiguous neighbour, and the world's most important production centre, the United States, with a substantively larger market of over 300 million (Grant \& Wood, 2008).

Temporal, Spatial and Institutional

Canada's relationship with its American neighbour is its most significant media relationship, and one that has influenced the course of its media policy from the introduction of mass media. In the early days of broadcast, due to geographical proximity, Canadians were able to tune their antennas to receive American signals (Skinner, 2008). They developed a taste for high-quality, American programming. To then produce a show of similar visual and written quality is much more economically problematic for Canadian producers, than for American ones. The small domestic market means fewer opportunities to amortize the sunk costs of production across 
viewers (Grant \& Wood, 2008). Broadcasters thus prefer to purchase Canadian territory rights to already-produced American primetime programming, which provides the high-budget content viewers prefer without the immense expense required to produce original content of a competing quality (Grant \& Wood, 2008; Hickling Arthurs Low, 2010; Vipond, 2011). This has been the plight of Canadian original production for decades.

Coupled with major production partners that have little incentive to invest in the risky process of original program development, the scheduling of Canadian content on television has also been problematic for similar reasons. The policy of simultaneous substitution, introduced in the 1970s, has acted as incentive for Canadian broadcasters to prioritize the programming schedules of American border over-the-air stations (Armstrong, 2010; Vipond, 2011). The policy was designed to allow Canadian rights holders to repatriate advertising revenues from licensed American content by requiring Canadian cable distributors to replace the signals of border American over-the-air channels with Canadian ones (including Canadian advertisements) when the American networks are broadcasting the same programs as the Canadian ones. In order to prevent the audience fragmentation that would occur if American and Canadian channels were to air the same content at different times, Canadian broadcasters mainly follow the programming schedules of American networks, relegating original Canadian 
content to the periphery of the primetime schedule (Armstrong, 2010; Grant \& Wood, 2008; Vipond, 2011).

Institutional and Social

Broadcaster control over the kind of content that successfully moves through the television production pipeline has engendered a system in which certain kinds of stories and perspectives have effectively been filtered out. Media scholars have long demonstrated the problematic nature of the dual product logic, whereby content companies produce programming for both audiences and advertisers, which in turn skews the content toward the demographics that have been deemed the 'most desirable' (Cooper, 2005; Croteau and Hoynes, 2001; McChesney, 2008; Meehan, 2006; Meehan \& Torre, 2011). Visible and ethnic minorities and their stories have been shown to be underrepresented on the Canadian screen, and when portrayed, they are often either stereotyped or their identities are neutralized so much that they appear rootless (Fleras, 2011; Murray, 2002; Solutions Research Group, 2003).

Women and visible minorities are also underrepresented behind the Canadian screen in all aspects of the field, especially in top creative decision-making roles (Coutanche \& Davis, 2013; Coutanche, Davis \& Zboralska, 2015; Davis, Shtern, Coutanche, \& Godo, 2014; Kaye \& Davis, 2011; WIFT, 2012; WIV, 2015), have been shown to earn less on average than their non-minority counterparts (CMG, 2013b; WIFT, 2012), and their growth in representation in the Canadian screen industries has 
lagged behind their growth in the general workforce (WIFT, 2012). The Canadian television industry has historically been, and continues to be, dominated by white males. The lack of risk-taking in Canadian media firms exacerbates the situation, with those in hiring positions relying on established professional networks, which tend to exclude women and visible minorities (WIFT, 2012; Davis, Shtern, Coutanche, \& Godo, 2014). This perpetuates the problem of underrepresentation, and leads to the maintenance of the status quo.

Barriers to entry in television broadcasting are high: those wishing to start a broadcasting enterprise must obtain a license from the CRTC to operate, which requires immense institutional and legal knowledge, and access to networks and capital. Even after a license is obtained, negotiating the terms of carriage of an independent broadcasting channel on incumbent-owned distribution services is a challenge, since vertically and horizontally integrated incumbents have both the capacity and incentive to prioritize their own slate of channels (IBG, 2016).

Institutional and Spatial

The Internet now presents an alternative and unregulated space for both access to television and television-like content from a viewer's perspective, as well as a new platform for Canadian creators to reach and engage audiences, and to monetize their work. Through distribution over the web, creators can now access audiences directly without having to negotiate the terms of access, sacrifice creative control, or obtain the 
permission of the majors. They also have access to a global playing field, and are, in theory, no longer limited by the small size of the Canadian market. The shifts brought on by the web are also beginning to dislodge longstanding institutionalized practicesincluding the primary Canadian broadcasting business model of importing American content, since American-based companies may now reach Canadian consumers directly by going over-the-top. Nearly half of all English speaking Canadians now subscribe to American-based streaming firm, Netflix (MTM, 2016), which competes with the majors for rights to programming. There are few domestic buyers of Canadian scripted digital series. Canada's sole standalone mainstream streaming service that does not require a pre-existing subscription to traditional cable or satellite services is Bell's CraveTV. Canada's other standalone OTT service, Rogers' two-year-old Shomi, shuttered in November 2016. CraveTV has thus far focused on the acquisition of primarily US-based popular programming (as did Shomi), and appears to have commissioned only two original scripted series to date including "Letterkenny", based on an independent sketch-comedy web series (Bell Media, 2015), and "Russell Peters is the Indian Detective" (Bell Media, 2016). OUTtv, an independent broadcaster aimed at the gay market, has recently launched a beta service. CBC is likely Canada's primary purchaser of original, short-form digital series, but the programs licensed have thus far only been comedies, and the licence fees paid are very low, often in the $\$ 10000$ range. 
Table 1 below thus provides an outline of the major contextual dimensions and elements that undergird the creation of scripted, screen-based content in Canada. While these elements are not exhaustive, they provide a big-picture overview of the source of the major conditions and constraints that Canadian web creators desire to break free from, and/ or to break up. The category of dimension is made clear, followed by the unique, influencing elements contained in that category, as well as the consequences that these elements have on the professional world that Canadian creators inhabit.

Table 1

Contextual Dimensions of Television Production and Distribution

\begin{tabular}{|c|c|c|}
\hline Dimension & Elements & Consequences \\
\hline Spatial & $\begin{array}{l}\text { Geographical proximity and shared language } \\
\text { permitted socialization of Canadians to US } \\
\text { high-production value content through over- } \\
\text { the-air programming. } \\
\text { The size of the Canadian domestic market is } \\
\text { small. }\end{array}$ & $\begin{array}{l}\text { Decreased demand amongst } \\
\text { Canadians for more modest- } \\
\text { budget, domestic content. } \\
\text { Small domestic market discourages } \\
\text { broadcasters from green- } \\
\text { lighting bigger-budget } \\
\text { productions. } \\
\text { Broadcasters prefer to purchase US } \\
\text { already-made product than } \\
\text { make risky investments into } \\
\text { original Canadian production. }\end{array}$ \\
\hline Temporal & $\begin{array}{l}\text { Canada's longstanding relationship with the US } \\
\text { set the stage for the protectionist cultural } \\
\text { policies that exist today. }\end{array}$ & $\begin{array}{l}\text { Policies to protect Canadian } \\
\text { cultural interests led to extreme } \\
\text { levels of consolidation in the } \\
\text { domestic media. system. }\end{array}$ \\
\hline Institutional & Policies are designed to overcome US & Policies create disincentives to \\
\hline
\end{tabular}




\begin{tabular}{|c|c|c|}
\hline & $\begin{array}{l}\text { geographical proximity and to permit } \\
\text { domestic advertising revenue generation on } \\
\text { US-aired content (simultaneous substitution). }\end{array}$ & $\begin{array}{l}\text { product and process innovation. } \\
\text { Canadian content relegated to } \\
\text { peripheries of primetime } \\
\text { broadcast schedules, } \\
\text { decreasing its visibility. }\end{array}$ \\
\hline & $\begin{array}{l}\text { Canadian industry is horizontally and vertically } \\
\text { integrated: a handful of companies control } \\
\text { internet, cable, mobile and broadcasting } \\
\text { assets. }\end{array}$ & $\begin{array}{l}\text { Massive consolidation limits the } \\
\text { number of buyers for Canadian } \\
\text { content. } \\
\text { Broadcasters make assumptions } \\
\text { about their markets; diverse or } \\
\text { alternative programming at } \\
\text { disadvantage. }\end{array}$ \\
\hline & $\begin{array}{l}\text { No culture of non-broadcaster private } \\
\text { investment in the Canadian screen industry. }\end{array}$ & $\begin{array}{l}\text { Industry is reliant on government } \\
\text { funding; producers require } \\
\text { broadcaster agreement to } \\
\text { access government money } \\
\text { (asymmetrical power } \\
\text { relationship). } \\
\text { Guaranteed funding disincentives } \\
\text { risk-taking. }\end{array}$ \\
\hline & Web space is unregulated. & $\begin{array}{l}\text { Canadian creators have new access } \\
\text { to distribution and audiences; } \\
\text { US over-the-top companies gain } \\
\text { direct access to Canadian } \\
\text { consumers, competing with } \\
\text { broadcasters for programming } \\
\text { rights. }\end{array}$ \\
\hline Social & $\begin{array}{l}\text { Over-abundance of qualified screen workers } \\
\text { exists in Canada despite small domestic } \\
\text { market. }\end{array}$ & $\begin{array}{l}\text { There is easy substitution amongst } \\
\text { workers; rates and working } \\
\text { conditions poor; competition for } \\
\text { jobs intense. } \\
\text { Those with connections to elite } \\
\text { networks and the right social, } \\
\text { economic and cultural capital } \\
\text { are privileged. }\end{array}$ \\
\hline
\end{tabular}




\section{Motives}

Although individual circumstances varied, a taxonomy of motives emerged from an inventory of the reasons provided by Canadian creators for their engagement in web-first production. The motives are presented below, and then contextualized against the backdrop of structures introduced in the previous section. The motives reveal deep insights into the kinds of constraints Canadian creators are reacting to, and attempting to both overcome and break up through their entrepreneurial ventures. The motives come from both explicit statements uttered by interviewees, as well as deeper, more covert or implicit ideas unearthed through the many hours of conversation held between the researcher and the participants, and thematic analysis of these conversations.

Web creators cited multiple, intersecting motives for their engagement in digital-first production. Not a single interviewee cited only one reason. The taxonomy below represents the motives of independent creators either working for themselves or for non-broadcaster affiliated production companies. The majors are a fundamental part of the institutional scaffolding that forms the sandbox within which independent Canadian creators work, and are, as will be revealed, a source of many of the constraints, which these creatives strive to overcome and change through their entrepreneuring. The motives are as follows: 


\section{i. To Overcome Canadian Industry Endemic Problems}

In Canada, the networks here don't do enough stuff. Yeah, there's not the same population [as in the United States]. Yeah, there's not the same amount of money, whatever. But still, it's like you can't just rely on the Americans because we're dead. We're gonna be dead. The world is global now, you need something that's gonna stand out. (Production company executive)

The large majority of independent creators interviewed, identified the lack of opportunity in the Canadian system as a reason to engage in digital-first creation. This was true for creators across all career stages. One established web creator expressed the problem with the Canadian industry as an institutionalized and systemic apathy:

There just really is an institutional dysfunction going on... [The broadcasting executives] don't want to get promoted, because they know there's a higher chance of them getting fired. So they're dodging promotions. And [broadcaster] is rotting from the inside out because media's changing and they're not. I don't know who cares in this business. Who's the person whose responsibility it is and who is actually steering the ship? I don't know their name. I don't know their position. I don't know their title. I don't know their phone number. I don't know their email address. I don't know if they exist. (Independent creator)

Another web creator expressed the problem through an analogy:

You go into a convenience store that's running a drug ring at the back, and you're upset that they don't have soup. You're like, 'Why is there no soup? What, you guys aren't even going to have soup here?' And they're looking at you, 'Dude, that's not what we do here'... There's all of these things, in Canadian television that make you really wonder. They don't wanna make it, they don't wanna do it. (Independent creator)

Many creators shared stories of pitching to Canadian broadcasters to no avail.

One production company executive shared his frustrations that despite being 
partnered on a project he was pitching with award-winning comedians, there was

simply no interest from Canadian broadcasters:

[The comedians]...they're friends. They had this idea and right away we responded to it and optioned it, and then initially we made a demo and shopped it around for TV. We didn't get it off the ground. It's certainly not the first and only show that we've had turned down. There are limited opportunities for TV in this country and it's a risk making a show like that with relatively unknown people even though [our partners] had won Geminis a few years earlier with [an earlier series]...which led to... really nothing, which is ridiculous 'cause in the States it'd be completely different. You'd have people coming up to you, being like, 'Oh, my god, what are you doing next?', whereas you talk to those guys and they say at the Geminis, it was just a joke. They win these two big awards and no one talks to them. It's weird. Anyway, so I think it was just like anything else. It was too big of a risk, it was too weird, whatever. (Independent production company executive)

This notion that the Canadian television industry suffers from an extreme aversion to risk was shared by many participants. Creators in the early and mid-stages of their careers were especially critical of what they perceived to be a lack of opportunities to engage meaningfully in professional production. Creators identified the small number of professional, scripted television series in development and production at any given time as a missed opportunity:

[T]he biggest issue is that not enough people get opportunities. For one, there's, how many shows that get made in Canada? And two, they don't give new people opportunities to make shows very often. I am sure it does happen, but it doesn't seem like the door is open. And the other problem is...the thing that I have noticed just trying to sift through the grant process for years, trying to see what was available-there doesn't seem to be any structure to how we develop talent in Canada... How is anyone going to learn how to make a television show? (Independent creator) 
Others, especially those at the very beginnings of their careers expressed a deep sense of discouragement over their prospects in the television industry, and expressed uncertainty about the stability of the industry in the future. Some pursued graduate degrees in the hopes of bettering their career prospects. One such creator commented:

I desperately have been trying [to find a job in television] since I've gotten out of undergrad, but no. So out of undergrad, I took a social media job with a small French language organization that was nearly a year-long contract. After that, six months looking for work, then I got a digital marketing job with a bilingual, national organization...After a while, it was just, 'This isn't really going anywhere. I'm still not in the industry that I wanted to be in'. So I started looking for jobs, and none were coming up, at which point, I was like, 'Maybe I need more education'. So then I went for a Master's... it came to the point where I'm like, 'Well, my undergrad hasn't given me a job in the field that I was supposed to get into. Let's see if the Master's is gonna help'. And frankly, I'm starting to realize, 'Wait, no. The Master's won't really help at all. It's actually being able to show that you've done something'. (Independent creator)

These early career stage participants felt let down by not only the industry, but also the larger television pipeline, noting that they felt that higher education had ill-prepared them for the truly volatile, unstable and problematic ecosystem that is Canadian television.

That the Canadian television industry is risk-averse is consistent with Berkowitz (2016), who found similar perceptions amongst high-ranking development executives working in Hollywood and Toronto. The interesting finding here is that the institutional apathy and aversion to risk, which impedes the development and growth of many 
creators' careers across all stages, leads some individuals to engage in entrepreneurial activities. There was a strong sense amongst many participants that the Canadian web series industry is, to a great extent, populated by many who were simply tired of waiting for opportunities, and decided instead to create them. One creator explained that his decision to engage in the web was linked to a frustration with the complicated nature of sourcing funding for television production in Canada:

So I, probably about two to three years out of university, started doing screenwriting courses, play writing courses... And I was accumulating these scripts, and accumulating these projects and these ideas, and I kind of wanted to see them realized. But the process of funding and the process of pooling together money for, even just a short film in Canada, is incredibly complicated. So it's often unless you're partnered with a producer who knows what they're doing, or if you kind of have some kind of industry connections. And at this point in my career, I did not, and I was very new to everything... But I had a friend who was in the industry, and he was more technically inclined, or is more technically inclined. And he said, 'Well, why don't we just pull this together, and why don't we use the web as just an outlet for us? It's our chance to just do this'. And I thought about it and I said, 'Well, yeah'. (Independent creator)

Another creator explained that the lack of meaningful opportunities to develop key skills in the traditional Canadian television system led him to create online series noting, "[f]or us, that was kind of what we wanted to get out of it actually—to be able to learn how to write and make a show" (Independent creator). A creator in his early 30s explained that he turned to the web after failing to attract interest in a pitch for a television series aimed at his generation: "I just put all my effort into YouTube and the Internet... mostly because there was no opportunity and TV has gone to boomers 
because they don't trust our generation to watch. So it's really hard to pitch a show aimed at millennials because nobody wants to make it..." (Independent creator). His failure to attract a buyer for his series targeted at millennials led to his taking the show online.

As one established creator observed, "...it's that sense of frustration, of not having enough places to take your work. So you end up with this bottleneck of talented people that erupts and all this stuff comes out" (Independent creator). This view of Canada as a place where underutilized and unexpended talent and energy is "bubbling up" and "bursting at the seams" was echoed by many of those interviewed. Another established creator made the connection between the web series phenomenon and Canada's traditional problem of 'brain drain' to Hollywood:

...web series are built off of people that have the creativity but no other outlet to channel it. The same reason that we do so well in comedy and all of our comedians move to Hollywood and become successful there, because that's where they're able to outlet that talent. And so, now that we're able to outlet that talent at home, it's happening through the web. (Independent creator)

Rather than moving to Hollywood to pursue the best opportunities (Berkowitz, 2016), many talented Canadian creators inevitably remain in Canada, and use the web as an outlet for their work. As will be revealed later, there are other constraints that appear to be leading to the brain drain of Canadian web-based talent and business to the United States. The web, therefore, has not stymied the phenomenon completely, but has been perceived by Canadian creators as enough of an opportunity to stay for 
the time being. The idea that the Canadian web series industry produces well-

regarded, high quality work was substantiated by Nordicity (2014), which found strong performance and screening of many Ontario-based web series ${ }^{4}$ at international award shows and festivals. Another established web creator, while also confirming how much 'easier' it is to make television in Los Angeles, suggested that Toronto-based web series creators have a unique opportunity to create strong online content:

I think in Toronto, we have a unique opportunity to do it [engage in web series creation] because the types of stories that people wanna tell, there's no outlet in Canada, there's just not...I don't think that any web series that I know of is a web series where they pitched it to TV, TV said, 'Sure, we'll do it'. And they said, 'No, we're gonna do it as a web series instead'. Most people who do web series either say, 'Why would I wanna go and try to convince this guy to do it', or they've tried to pitch it and got turned down enough times and they're like, 'Fuck it, I'm just gonna do it...'. (Independent creator)

It is apparent then that some web creators, through their entrepreneuring, are striving to overcome constraints in the Canadian system, and to create new pathways to success. For others, the web is seen as a less complicated path to the realization of a creative project or dream. As one established web creator explains,

It's much easier to get a project off the ground on the web through the IPF than it is to get a television series going.... I personally don't like ...the idea that $90 \%$ of what I write will never be produced. I don't like the idea of being in development for two years, writing a bunch of scripts. And then, not even going to pilot...[A]ll of those funds, unlike the IPF, you need a Canadian broadcaster to sign on it, and put in some money... (Independent creator).

\footnotetext{
${ }^{4}$ The category, "web series", in the Nordicity (2014) study was more broadly defined than in the current one, and did not disaggregate scripted web series from non-scripted.
} 
Another creator with an earlier stage production firm that is well known in the digital sphere confirmed this idea, noting that his firm is pragmatic about the situation.

Although his company has become known for its digital series, it's not their "...MO to make digital series", but the medium provides an easier pathway to "get content made", although their preference is to get "some of that TV money before it goes away".

Thus, although there are fewer sources of funding for Canadian digital-first productions, and these funds provide much lower allocations than funds for traditional television—for those more established or industry-savvy creators, it is still easier to compete for digital series funding than to get something produced with the majors. Many of the creators interviewed, therefore, turned to web series because there were fewer barriers to securing funding than in television. They made their web series as a way to create a proof of concept for television, something they would never be able to do otherwise.

Many web creators also saw the web as an opportunity to separate from what they perceive to be a system on the decline. This was especially true of executives at established independent production firms who identified the need to be more global as an issue of extreme importance. One independent production company executive put it this way, "[F]or us, when you're talking about doing stuff on digital...you want to reach as many people as possible and you have that ability" (Independent production 
company executive). Jay Bennett, Senior Vice President, Creative and Innovation of established Canadian independent production firm, Smokebomb commented, "I think you have to be global" and stressed that unlike the majors currently do, Canadian producers must think of their audiences as international as well. Frustrated by the persistent lack of sustainability in the Canadian scripted sector, he sees the web as a potential "way off the highway" that is the usual cycle of dependence built on government grants, subsidies and broadcaster licenses. His company has relied on private sources, notably, partnerships with brands, to fund their scripted online series. Another established production company executive appreciates the absence of regulatory constraints in the online space, insisting "There are no barriers anymore. I don't have to deal with the CRTC. I don't have to ask anybody's permission" (Independent production company executive).

That Canadian creators turn to the web in search of emancipation corroborates previous research on American-based web creators by Christian (2012a), who discovered that many of these creators turn to the web to overcome the constraints and barriers in the Hollywood labour market. Although the particular contextual circumstances vary between both American and Canadian markets, it appears that individuals are turning to new distribution technologies and engaging in entrepreneuring, however uncertain, to carve out new paths for themselves-to both overcome, and break up the status quo. 


\section{ii. Identity, Marginalization and Social Good}

Many people have been asking me what do I want to do with my life. If I could be an actor, just an actor, I would be. But, the roles don't exist. It's like Viola Davis says, 'You can't win an Emmy for a role that doesn't exist'. And so the choice of me just being an actor, unless I want to be store clerk number two, it's actually not an option for me...So now I'm like, 'Alright, let's create some content'...that's what I have to do. (Samantha Wan, Independent creator)

Although the lack of opportunity in traditional television broadcasting was noted by many creators, those from traditionally marginalized groups including visible, ethnic and religious minorities, minority language communities, individuals who identify as women, and LGBTQ, sometimes expressed more personal senses of frustration with the system. Many expressed feeling let down by the traditional industry in some way, and perceived the web to be an enabling platform allowing them to take control. Creators in this category expressed impatience and unease with waiting for opportunities, and opted instead to create them. Many of these individuals had been trying for years to break into the Canadian television system as actors, for example, only to be offered stereotypical roles (ex. the police officer; the geisha; the store clerk). Creators in this category, therefore, see the web as an opportunity to present themselves in a new lens-through the kinds of roles they have rarely, or never, been offered.

Tino Demitro, a creator of mixed descent, and of an unidentifiable visual ethnicity, expressed his frustration this way: 
I would meet with agents and they would sit there and they'd look at me and they'd say, 'I don't know what to do with you'. I don't know where you fit in this country, in this city. You're not East Indian, you're not Western, you're not Asian, you're not black, you're not... What are you? Are you Italian? (Tino Demitro, Independent creator)

After being consistently typecast as prison guard or police officer, Tino saw the web as an opportunity to present himself as a comedian, something he believed he was particularly talented at.

Another creator of mixed descent, Yusuf Zine, reported similar experiences to Tino, being submitted for stereotypical roles by his agent, but rarely selected because he was not ethnic "enough":

[A] lot of the auditions I was being called out for as I got older started to become very stereotypical, like the terrorist roles came in a lot. Because my resume said I was part South Asian, I got the typical Indian guy as a kind of goofy typical guy. And I never got any of those roles cause I was never really 'brown' enough. I was never really like 'terrorist-looking enough', so it was...frustrating.

Yusuf explained that he viewed his web series as a way to challenge the status quo, and to re-educate viewers about stereotypical representations in the mainstream media:

For me it was really just trying to really challenge some of the dominant narratives of minorities, and hopefully it would having this be an opportunity to get the show out there so we can have a conversation about our industry right now and how people are so... Like I always said, whenever I talk about the show, if one person watches this web series and then goes and sees a movie where there are terrorists and stuff, and realizes that's not all Muslims and Arabs...if they kinda have that little realization, then I feel like we've done our job...[T]here's just so many films out that kind of still paint certain ethnicities the same way. So if people can start to realize 
that this is problematic, this is not representative of all minorities, then I think that is a great outcome for the show. (Yusuf Zine, Independent creator and startup independent production company co-founder)

J.P. Larocque, a gay creator, was frustrated by the inadequate, and stereotypical portrayal of gay characters in the mainstream media:

[S]o you have a gay character on a show, and it's like they're only a template of two to three gay characters that you can see. So, for me I was like, 'Well, what about a nerdy gay? What about these characters that we don't necessarily see represented on television yet?'

This frustration formed part of his motivation to create a web series about gay men and women who also happen to be "nerds". When pitching the show to broadcasters initially, he was met with rejection:

It's gay characters as main characters and oftentimes, and especially in the years since launching the web series when I've pitched it to networks, and to broadcasters, and wherever, the reaction in general that l've had is like, 'This is niche story telling, we don't really have a platform for this'. Like, 'Do we really need to tell a gay-specific story?' There's been more resistance. (JP Larocque, Independent creator)

He realized that, "Online didn't do that, there were no gatekeepers. So I kind of was able to just tell the story that I wanted to tell", and subsequently launched the series himself on the web. After finding considerable success with audiences online (although not economic success), the series has recently been picked up by the Canadian gayfocused independent broadcaster, OUTtv. 
Another creator shared similar experiences shopping her project around the majors: "So, one of the main aspects of my show was $[A]$, female leads and $[B]$, they're gay...that did not fly with broadcasters that we were approaching" (Independent creator). She found that with broadcaster interest came limitations on how much she could challenge mainstream representations:

[T] hen as we shopped it around, it was like, 'Well, if we could change this, or if we could change that'. It's like you see all these changes coming, it's like, 'Ah, then that's not actually the show we wanted to do'. (Independent creator)

She preferred to keep the project going on her own than agree to unfavourable terms with the majors. Another young female creator rationalized her decision to pursue an online series the following way:

[T]elevision and film are very particular about how they want people to look. So, if I was just an actress who had a really great web series idea, I think the industry is still very built on, 'Can we sell a woman sexually? Can she be a hot little commodity to put on television?' Which I don't think I fit into that at all. So for someone like myself to be able to get to the level of success on television, I think I would either have to be the funniest woman in Canada or the hottest. (Kate Conway, Independent creator)

For her, the lack of risk-taking on the part of the majors on women who do not fit the typical television mold, and the lack of general opportunities in the Canadian television system, led her to engage in entrepreneuring. For the P.E.I.-based creators of online series "Just Passing Through", the lack of representation of stories about Canadian Maritimers in mainstream Canadian media, as well as the lack of opportunity 
to tell these kinds of stories, formed part of their motivation to engage in online series creation:

And that was part of the show too. We made it about two guys from PEI going to Alberta, so there are personal elements... We've never done that personally, but we know people who have, and so there was a desire to tell an island story, and it features island characters specifically, right? ... it's not shown at all [in the mainstream Canadian media]. And that element of it, I think is another thing that would prevent it from being put onto a major broadcaster... They wouldn't make a show about two guys from PEI. (Jeremy Larter, Independent creator)

For other creators, although perhaps they have not been marginalized

themselves, a cause that they are personally connected with has been. Jewish creator,

Eli Batalion explained that part of his motivation for his web series was to serve as a

corrective to the mainstream media's depiction of Yiddish:

There's also a bit of an altruistic incentive, which was how to preserve [the Yiddish language]. Which, another thing I should just add about my background, is that on my mother's side, they were strongly Yiddish...there's a whole spirit to the use of Yiddish language...everyone who was this left-wing thinker in the Jewish community, they were all Yiddish speakers...Part of the reason why we do this show is because the only Yiddish speakers for the most part today are all ultra Orthodox Jews, that are the complete opposite. And it's also a bit of a demonstration to both the non-Jewish and Jewish community that that's not what Yiddish is....And it's very much a language of the secular... and a language of mixing a variety of cultures. In Israel, in particular, it's associated with weakness, and coming from a lack of selfdetermination. In America...it's a caricature. And that is a large way in how it's portrayed. Part of the motivation was to change that.

Others still explained that the web provided a way to overcome insufficiencies in their own professional networks, noting that they believe nepotism and favouritism is pervasive in Canadian television: 
[T]he reality is I was still an amateur filmmaker...I'd never made anything. I didn't know a single person at a network. I didn't know anything about the studio system... [My series] is not television because I didn't know how to get into television. (Independent creator)

Another creator echoed the same remarks:

No one's gonna give me money or broadcast time on television whereas that was not an issue with the web...I think, really because I didn't know, I wouldn't know who to go to...And maybe they would [give me money]... but I didn't have the contacts to do something broadcast wise that way... whereas, I did know how to make something and upload it. (Independent creator)

The frustration of these creators, and the pursuit to overcome their exclusion, or the exclusion of a cause that they are personally connected to, by the mainstream television media, therefore, formed a significant part of their motivation to engage in entrepreneurial activity. For many, starting a web series was perceived not merely as an opportunity, but rather, as a necessary step to overcoming the discrimination they believed to be at the source of their career stagnation, as well as an opportunity to challenge and change representations and viewpoints.

iii. Capacity to Connect with a Niche Audience

What I have found is so integral about web series, if you are looking for a fan base to support you, is to find a niche. You can't have really general content, because that audience is not going to support you. (Regan Latimer, Independent creator, co-founder, IWCC) 
Many creators and production executives see the web as presenting an efficient and effective way to target and reach niche audiences. A key strategy to finding success in the web space according to one long-standing web-first creator, and the current vice president of the IWCC, Dan Speerin, is "doing something the industry is not giving us". Many observed that genres with more broad appeal have already been 'tapped' by mainstream media broadcasters; these shows typically have larger production and marketing budgets, making it difficult for independent creators to compete in the same categories. Jason Leaver, creator of one of the longest running, and most viewed independent web series, "Out With Dad", explained:

One of the earliest things I remember her [Felicia Day, a US-based web series pioneer] saying back when I was researching—she said, 'If you have this idea for a show and it's about a bunch of white friends that gather in a coffee shop and say catty things at each other, it could be the best show ever made. But don't bother because NBC already made Friends and they've got more money than you so they're gonna do it better than you. Say what you will about the scripts. It's got better production and value and people are gonna watch that instead. So don't bother making that'. But what if that show was a person of colour in a wheelchair...and there's eight of them and they're in a coffee shop, then maybe that's a web series that would take off because there's an audience, they would rather watch that than Friends'. (Jason Leaver, Independent creator)

The web thus provided a viable entry point into the content space in a way that did not place him in direct competition with the majors, or Hollywood.

As independent creator Herman Wang put it, People who like reality shows, they've got a glut of it in the making it, so no one's going and doing that as a thing because well, I can just get it on the channels I already watch. Whereas, yeah, people who really like science fiction about this and 
that, it's like 'I can't ever find it on TV', so if you can figure out a market, a niche market that's underserved...

Speaking about niche web series "YidLife Crisis", IWCC VP Dan Speerin observed:

'YidLife Crisis' doubled down on the narrative of what web series can be because once all these things like Netflix and YouTube become bigger and bigger, where are you going with your show? I think you get buried. So if we're talking about what shows are gonna make sense in moving forward, it's going to be stuff that talks about marginalized communities and/or topics that don't get a lot of attention... niche topics.

These creators and firms recognized a tactical rationale for niche storytelling, strategically tailoring their aesthetics, ways of communication and sensibilities in both their content and in their outreach to groups that have been underserved. This involves, as one senior executive at an established production company put it, "speaking to them in their language" (Jay Bennett, Independent production company executive). According to several of the creators interviewed, the process requires thinking and conceiving of viewers not as audiences, as is the norm in traditional television—but as "communities" first.

Many creators thus recognized a business case for diverse storytelling, seeing the web as a potential platform to connect with audiences that might be hungry for content about their communities. Independent creator, Jamaal Azeez, explained the strategy behind his online series, which explores themes of both overt and covert racism through the journey of a young visible minority actor: 
[W]e recognized an opportunity that wasn't even strictly confined to one culture per se. It was this overarching theme of underrepresentation and misrepresentation which the more we started to talk about we said there's nothing like this out there, otherwise we wouldn't have this sort of frustration and we wouldn't be inclined to make this kind of show. It was very much informed by an absence. (Jamaal Azeez, Co-founder, Independent production company startup)

For Azeez, however, and the others who recognized the strategic benefits of producing for such underserved demographics, the decision to pursue more niche stories was not a business one alone. Expanding the scope of media representations was identified as an issue of vital importance for these creators. Jason Leaver's series is about a young lesbian teenager coming out to her father. He explains his reason for why he chose to tell the particular story:

And part of the motivation too was, given the nature of the story, there's a chance to make a difference in people's lives. 'Cause when I first started researching, a lot of what I was finding was very negative. It was like 'how to cure homosexuality' and you know, it was all of this terrible stuff and I was just... When I was reading, when I was putting myself in the position as a parent, suspecting my kid was gay or I was Googling all that stuff, the results I was getting were very disappointing. And so that really means, 'okay, well then I have to make this show 'cause I want a positive example', and so that's what happened. And so, if I made a feature that nobody sees, and if I pitch a TV show that'll never get green lit, or make a short film that no one's gonna see than what's the point?...At that time, I've actually found, I remember reading the top ten most offensive things that Americans will see on television, the number one thing was lesbians...the reason 'Out with Dad' was lucky, is because it was about a girl coming out and that content wasn't there elsewhere, and so people were seeking it out. (Jason Leaver, Independent creator)

Sarah Shelson, a young female creator with a digital-first start-up, who felt strongly about the lack of representation of certain groups, incorporated characters from 
several diverse and traditionally underrepresented demographics into her series,

including individuals who identify as asexual. She said this led to her building a strong

and passionate following:

I think the reason these young people...are getting into that web sphere, is because they're not seeing their stories anywhere else, right? So then if they're not seeing it anywhere else, they want to see it here. They don't want to come here and just see television, because they have television, they have movies. So the web sphere is very much like, 'Find your niche audience', and part of that are these communities like the asexuals, who were like... a lot of them are like, 'This is the first story that showed it on screen where this is actually legitimately part of the series, an asexual character. And this is super important to us'.

Another established independent creator linked his decision to cast a South Asian female in a lead role in his series with a desire to connect with the Indian market: "I specifically made the protagonist a south-Asian female because I thought that it would be an opportunity to reach that market...I was really hoping to reach India and much broader" (Independent creator).

Recognizing an appreciation for diversity in the web sphere, and a desire to do good, another established creator remarked,

We did [cast a visible minority]. That was somewhat strategic in the fact that we did... And I believed it was important to put on a non-Caucasian face as our lead. And then once we'd done that, the trickle-down started to casting our family, to casting [the rest of the show]. (Jay Bennett, Independent production company executive)

Creators thus revealed a complex mixture of both social and business-oriented goals as part of their motivations to reach and target niche audiences. All creators who pursued 
a niche online market agreed that the topic must be one that they know well, and they are passionate about. Experienced broadcaster, and currently, co-founder of a developing OTT platform and studio, Jeffery Elliot, explained his decision to create a streaming service that targets guitar, motorcycle and aviation enthusiasts because these are audiences that are not only underserved, but topics that he and his partners know well. They were after,

...things that we would be passionate about and things that we would understand and things that we know to be authentic about... [His major concern was] that we're not just doing it 'cause, 'Oh hey! It's a good idea. Let's do motorcycles'. You actually really, I think, have to know the subject and be able to articulate and understand. (Jeffery Elliot, Independent production company executive)

This idea of the necessity of genuinely understanding a niche was a key sentiment shared by creators who pursued niche storytelling.

\section{iv. Creative Autonomy, Experimentation \& Authenticity}

I'm approaching it not so much from a strict business model but as an artist. This is what I do. This is the kind of work that I do and some of it's more experimental, some of it's more straightforward narrative... But, I'm not doing it...for the recognition of it- I just want to do it because I can. I feel like doing it. I don't want anybody telling me no, or you have to change it. (Rob Mills, independent creator and co-founder, IWCC)

Many creators cited the creative autonomy that comes along with producing for the web space as a key motivating factor to engage. Established creators reported seeking respite from the usual process of network notes and rewrites. Scott Albert, a successful 
writer in the traditional television industry identified achieving independence as a long-

term career goal. The web offered that type of creative freedom immediately:

[D]oing stuff with the web was a way to be entirely creatively independent. And to just write what I wanted to write and do the shows I wanted to do, without having to wade through a lot of notes from the broadcasters and production companies. (Scott Albert, Independent creator)

Herman Wang, a computer programmer, uses his web series, "The Spell Tutor", primarily as a creative outlet. The series is based loosely on the world (and intellectual property) of Harry Potter, and therefore can never be a for-profit endeavour: "The web is the ceiling... it's a creative outlet and as long the creativity is still there, and the inspiration is still there, then I'll keep doing it" (Herman Wang, Independent creator), he notes. An established web creator with a popular web series reported engaging in web series production after feeling creatively unfulfilled with his prior job as a corporate videographer, even though it paid well:

They weren't my stories. I was telling third quarter result stories. And so I just thought to myself, is this my lot in life just to do this? I kind of had to decide, I was on the verge of saying, 'Yeah, I'm good with this, I won't get to tell my stories'... it was very much a creative fulfilment endeavour, this project. (Independent creator)

Other established creators reported seeing the web as providing a unique opportunity to experiment with interactivity and to defy usual televisual conventions and norms in terms of both content and style. Accomplished television writer Jill Golick noted that the web allows a creator to establish a unique relationship with the audience: 
[W]hen you are on the web and on YouTube and on Facebook and on Twitter and on Instagram, you know your audience in a very different way because you know, they comment on your work. And then you could go back and find out who they are...you can watch their YouTube channel. You can follow the links to their Tumblr. You can know them in a very personal and intimate way that broadcasters can't possibly know their audience because it's too big and it's so separated by the cable and the distance.

This subversion of the traditional creator-to-audience relationship is a unique aspect of web-based production.

Another creator cited the opportunity to experiment and learn in a lower stakes environment as a motivating factor behind his engagement in web creation, an opportunity not available in television:

I think an important aspect was the experimental opportunity...some people are driven by an opportunity to do something perfectly in a high stakes, high pressured way. But for me...I need to go in in a low stakes, relaxed kind of way to experiment with something, to build up skills and whatnot. And so, yeah, the idea was this would be great, this would be a good learning tool...this is film school. I didn't go to film school. (Eli Batalion, Startup independent production company co-founder)

For him, producing for the web also allowed him to grow the kinds of skills he had never had the opportunity to develop having never gone to film school. Another emerging creator echoed the notion that the web provides an excellent opportunity to develop skills and to tell the story he wanted to tell, in the way he wanted to tell it:

So I realized...I needed to either try to get in on the structure of mainstream TV, get in a room, climb that way, learn production stuff as they hand it to me, as I grow, or alternately go the web series route which is expensive, you make no money, but you are immediately in charge of your own fate. I produced for the first time even though it was terrifying, I ran casting, I was a show runner with nobody to answer 
to...I feel like it was partly just a creative catharsis, and partly I wanted to put something out there that was purely me. (RJ Lackie, Independent creator)

M.H. Murray, the award-winning young creator of the popular web series

"Teenagers", believes the lack of formal censors or ratings bodies in the web space

allows him to convey the experiences of youth in a more authentic way:

[W]ith a network, it's always just going to be cleaned up a bit. And it's kind of just really hard to really see through the glossiness of it. And what I think is good about Teenagers, is that it just... you can still tell it's like a web show, and I think that because it's like almost this dirtier version of Degrassi... It's weird, it's like this imbalance between violence and sex. Violence is allowed on TV, but sex isn't.

His series frequently depicts the kinds of scenes that are still taboo on mainstream television aimed at youth, including on-screen gay sexual encounters and sexual assault.

Creators also expressed satisfaction with not being bound by the conventional television episode length and structure. The episode lengths of the series produced by the creators interviewed greatly ranged in length. Creators generally rejected the need to follow a strict format. "Story determines length", one established creator succinctly stated.

Many of the creators interviewed connected their desire for an unfettered outlet for their creativity to their frustration with the risk-averse Canadian system, which according to these creators, does not encourage experimentation, and often gets in the way of it. As one established production executive observed: 
A broadcaster needs to appeal to these wide demographics. They need to adhere to very strict, 'This is 22 minutes. This is 43 minutes'. The luxury here is we cut when it should end. There are many stakeholders they owe to and the freedom of digital... I love this sweet spot we're in where we have the distribution portal, we can fund it through a brand, and we have the freedom to tell the stories the way we want to tell them. And I'd say that is the biggest difference, is there's so much money behind television series, there is very little opportunity to take risks... Especially in Canada, they're not able to take a risk when they're gonna order one show for the year. (Jay Bennett, Independent production company executive)

Another independent creator reflected on the difference between producing for the web, and then subsequently producing for television:

So first of all, television is a different art form, completely. And we were very free form on the web, right? But for television, we had to learn how to write a television show. So we hired a story editor that basically said, 'Okay, well, this is a three act structure. There's an A and a B story. The stakes have to raise before the commercial break every time and there is no getting around that. That is set in stone. The network will not accept a script that does not have those criteria'. (Independent creator)

Another established independent production company executive similarly observed, "There's certain handcuffs, if you will, working with a broadcaster, and you are really beholden to their schedule on when they wanna release and how they wanna promote it". The desire for experimentation, and creative autonomy were thus significant motivating factors for engagement in web series creation. The web space gives creators the opportunity to tell their story their own way without diluting their vision. This establishment of an undiluted voice is a potentially powerful method of career entry for early stage creators, and offers established creators a space to experiment, 
play and a respite from the industrial hierarchy and conventions they face in their everyday working lives.

iv. Economic Goals

No interviewee cited profit as the sole motive driving their entrance into the web space. When profit was cited as a motive, many creators were seeking economic sustainability rather than to maximize profit. Early-stage creators, in particular, saw the web as providing the capacity to create "calling card" pieces that could lead to future potential profit generating ventures. This motive was closely connected with the desire to overcome the lack of meaningful opportunities for talent development in the Canadian system. These creators hoped that by creating high quality pieces with large audience followings, they could capture the attention of established media entities (both traditional and digital) for the funding of future projects (including potential webto-television adaptations) or opportunities to work in lead roles on other productions.

Creative partners Jeremy Larter and Geoff Read explained,

Jeremy Larter: I think the thing that we realized early on is that the only way to really make money is if you sold a show. If you sold the show or if a broadcaster picked it up, that's the only way that we thought that you'd ever make enough of a profit... To have a revenue source of any size would be to have someone else pick up the show.

Geoff Read: So the main goal was to just make something good enough that someone else would be interested in it, and then come onboard. 
Creator Hannah Cheeseman noted that for her team, profit was never a primary motive:

So, no there was not money in mind. Everybody I think who gets into this is hopeful there's gonna be money or hopeful that it will transform into something that can be creating money, but it was not a thought. It was, 'Let's just try and make something'.

As M.H. Murray reiterated, "We just wanted to make something that could help get our names out there". For most creators, therefore, immediate profit was not an expectation. Many did not expect to generate any economic return from their particular projects, but did expect the projects to lead to spin-off opportunities.

This does not mean, however, that no creator intended to eventually see economic returns on their particular web series. For some creators, revenue generation and profit were sought as part of a longer-term strategy. Emerging independent creator Alex Young explained that he hoped his series would gain enough of an audience that it could sustain itself through crowdfunding past its first season:

So the plan had been, season one is very simple, but we get our feet wet, and we get it off the ground. And we get just enough of an audience that we can justify a second season...Season one was supposed to be: 'We make it for nothing, so we don't go bankrupt when just our mom watches it. Maybe we get just enough of the audience... people on Facebook, whatever, that gives us the push and the drive that we can, like, call on 500 people, and be like, 'Hey, you guys, if you put in 20 bucks, we'll do the next season'. (Alex Young, Independent creator)

As will be made clear in chapters 6 and 7 , the generation of revenue and profit have been extremely challenging for Canadian creators for a variety of reasons including those related to the larger digital marketplace, as well as reasons endemic to Canada. 


\section{Discussion}

Understanding when, how, why and who engages in entrepreneurial activity requires a deep appreciation of the various contextual dimensions that undergird the entrepreneurial behaviour. Rindova et al.'s (2009) framework, which envisions entrepreneuring as fundamentally oriented at change-making, implies an in-depth understanding of the contextual elements entrepreneurial actors aim to break free from, and to break up. In Section 1, work from the critical political economy perspective provided a big-picture understanding of the many structural components that together form the world in which Canadian creators are situated. These elements are in no way stable or fixed, and individual creators use their agency in an attempt to stand up to, overcome, and/ or break up the various structural constraints that they perceive to be problematic in their environments by engaging in entrepreneuring through creation for the web. By examining their motives through the entrepreneuring as emancipation framework, the process of structuration, and its intricacies comes to the forefront. The struggle between structure and agency becomes that much more visible. Table 2 below breaks down the non-monetary motives cited by Canadian creators into the dual aims of 'breaking free from' and 'breaking up', and also highlights the contextual dimensions that are implicated in these aims. 
Table 2

Contextualization of Non-Monetary Motives for Entrepreneurial Engagement of Canadian Web Series Creators

\begin{tabular}{|c|c|c|c|}
\hline Motive & Breaking Free From... & Breaking Up... & $\begin{array}{l}\text { Contextual } \\
\text { Dimension } \\
\text { Implicated }\end{array}$ \\
\hline $\begin{array}{l}\text { To overcome } \\
\text { Canadian industry } \\
\text { endemic problems }\end{array}$ & $\begin{array}{l}\text { Limited opportunities in } \\
\text { Canadian scripted } \\
\text { television system } \\
\text { Canadian system that is } \\
\text { perceived to be on the } \\
\text { decline }\end{array}$ & $\begin{array}{l}\text { System that perpetuates } \\
\text { limited opportunity } \\
\text { through the creation } \\
\text { of new ventures } \\
\text { Canada's limited role on } \\
\text { the global media } \\
\text { stage }\end{array}$ & $\begin{array}{l}\text {-Institutional } \\
\text {-Spatial }\end{array}$ \\
\hline $\begin{array}{l}\text { Identity, } \\
\text { marginalization and } \\
\text { social good }\end{array}$ & $\begin{array}{l}\text { Limited opportunities for } \\
\text { women, visible } \\
\text { minorities, and other } \\
\text { traditionally } \\
\text { marginalized groups } \\
\text { Limited opportunities for } \\
\text { diverse storytelling } \\
\\
\text { Absence of, or inaccurate } \\
\text { representations of, } \\
\text { minorities in/by } \\
\text { mainstream media }\end{array}$ & $\begin{array}{l}\text {-Casting and hiring } \\
\text { conventions through } \\
\text { self-initiated roles and } \\
\text { opportunities } \\
\text { System that perpetuates } \\
\text { underrepresentation } \\
\text { of diversity by putting } \\
\text { missing stories on } \\
\text { screen } \\
\text { Nepotism and elitism } \\
\text { through creation of } \\
\text { new networks and } \\
\text { opportunities }\end{array}$ & $\begin{array}{l}\text {-Institutional } \\
\text {-Social }\end{array}$ \\
\hline $\begin{array}{l}\text { Capacity to connect } \\
\text { with niche audience }\end{array}$ & $\begin{array}{l}\text { Inability to access and tell } \\
\text { stories for diverse or } \\
\text { niche audiences due to } \\
\text { broadcaster market } \\
\text { assumptions } \\
\text { Saturation of mainstream } \\
\text { media in broadly } \\
\text { focussed content }\end{array}$ & $\begin{array}{l}\text { Conventional media } \\
\text { thinking around } \\
\text { desirability of certain } \\
\text { demographics } \\
\text { Conventional media's } \\
\text { hold on access to the } \\
\text { audience }\end{array}$ & $\begin{array}{l}\text {-Institutional } \\
\text {-Social }\end{array}$ \\
\hline $\begin{array}{l}\text { Creative autonomy } \\
\text { and } \\
\text { experimentation }\end{array}$ & $\begin{array}{l}\text { Institutionalized } \\
\text { hierarchy/ gatekeepers }\end{array}$ & $\begin{array}{c}\text { Dominant power } \\
\text { structures and } \\
\text { creators' own }\end{array}$ & $\begin{array}{l}\text {-Institutional } \\
\text {-Social }\end{array}$ \\
\hline
\end{tabular}




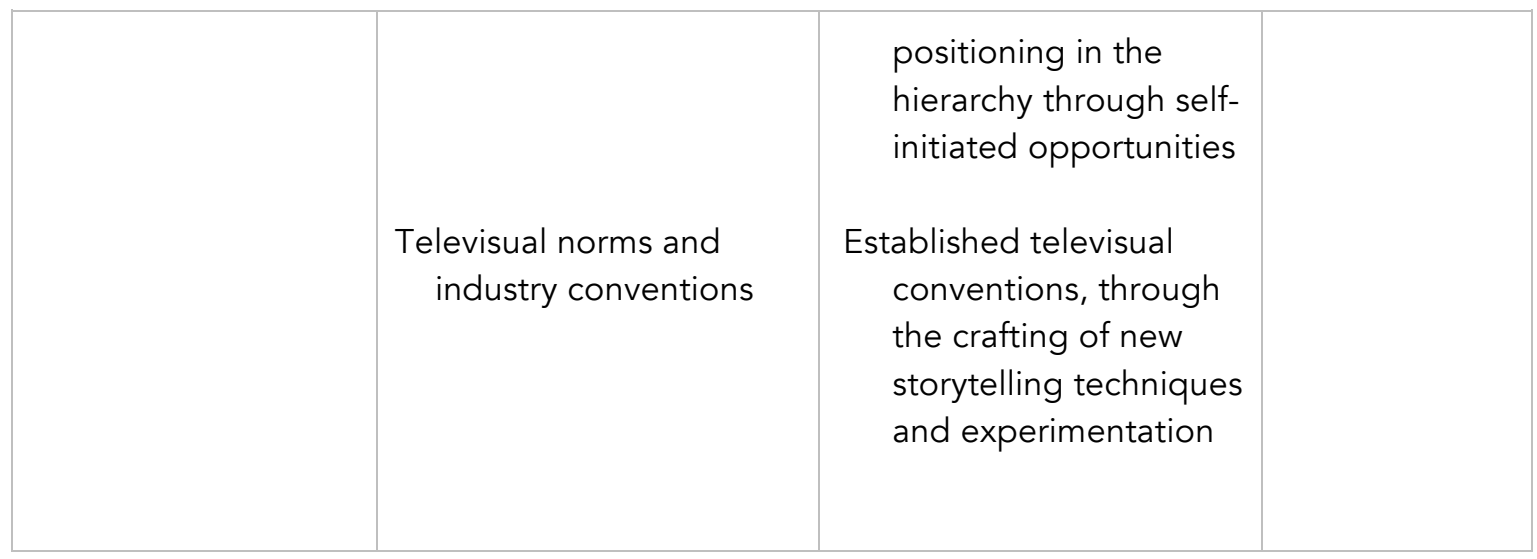

Canadian creators seek to initiate change through the creation of "a new idea, a new thing, a new institution, a new market, a new set of possibilities" (Rindova et al., p.478), for themselves, and in many cases, in the larger system in which they are embedded. This requires breaking free from the multiple, multidimensional, intersecting and shifting constraints that are binding them, and disrupting the status quo by initiating change for themselves and, sometimes, for others. For these creators, multiple structures and forces collide at once to create constraints in their worlds.

The creators who are inspired to engage in entrepreneuring due to the limited opportunities in traditional television, seek liberation from the various spatial and institutional restrictions which have led to these limitations, and hope to shatter these restrictions by overcoming the small Canadian marketplace, for example, by forging links to global audiences through online distribution. They aim to overcome and disrupt the systemic inertia stemming from negative externalities related to a long and storied cultural policy history, and Canada's unique relationship with the United States. 
Those who have felt marginalized, either due to their identities or the kinds of stories they wish to tell, want to break free from the institutional and social constraints that prevent their upward mobility or representation, and to break up these structures by creating the kinds of roles or stories they have not been able to pursue or see. They also attempt to overcome weaknesses in their own professional networks by creating work that they hope will bring them attention and meaningfully expand these networks.

Those engaging in online creation (and distribution) of their stories in a pursuit to connect with niche audiences seek to break free from mainstream media's institutionalized stronghold on the audience commodity, and aim to redefine the demographics that 'count'. The creators seeking creative autonomy through their entrepreneurial activities are looking to break free from and disrupt and dislodge institutionalized systems and industry conventions by building anew. By breaking free from institutional and spatial constraints in the Canadian system, they aim to elevate the system, and their role within it. This means for some, an opportunity to break away from certain televisual boundaries and norms, permitting them to experiment with aesthetic, storytelling and relationships with audiences. All of these motives exist generally prevail over economic reasoning.

The motives discussed here have historical context and were so strong amongst web creators that they coalesced into a movement to start a Canadian association for web creators in 2012. Creators who were tired of the status quo, wanted to share 
educational resources, and resolved to maintain the independence of the web, felt that their efforts might be better served if they organized together. What began as a group of 30 people meeting at a pub bi-monthly, eventually evolved into the legal nonprofit, the IWCC. This is currently Canada's only association for creators of online series. The organization's founding manifesto solidifies and brings together the goals and dreams of its early members. It reads:

We are Independent Web Video Creators, a newly founded group which represents the interests of creative professionals who craft and distribute video content across the world wide web.

We are connected directly to our audience via the screens through which we deliver our content. As creators we retain the rights to our work and govern its reach across the web. As artisans we engage with all disciplines to craft our stories. As storytellers we are in constant conversation with our audience, we establish communities and continue to build upon our creations.

We do not seek nor do we require permission to make or share our work.

The old structures of the media industry fail to serve our needs and we reject any filters or gatekeepers of old media which would stand between us and our audience. As independent web video creators and storytellers we share the vision of our work with our audience, using the democratic tools of the internet to create a richer, collaborative two-way experience.

We acknowledge the hard won protections of all players in traditional media (producers, writers, performers, musicians and more) contained in various agreements which are staunchly defended, and rightly so, by all concerned. We do not seek to unravel, negate or destroy those agreements. Instead, we are media professionals seeking to have the needs of this new generation of digital creators addressed with fresh minds and the clear understanding that creating for the web is a new industry unto itself, not an extension of the status quo, with a need for new working relationships and collaborations amongst all parties. 
We do not intend to be confrontational with any established group. We are neither foe nor adversary to old media. However, we are firm in our resolve that these are changing times in a rapidly changing world where the rules of engagement must also change - and for the better to all concerned.

We seek to govern our own fate. We are committed to real long term growth as a professional industry. We embrace new business models and new ideas.

Independent Web Video Creators are organizing throughout the world to share knowledge, experience, skills and resources. We gather online and in the real world to represent our needs and shape our emerging industry. To those who also embrace new ideas of narrative and distribution, to those who are creating a new relationship between artist and audience, and to those who are explorers and innovators in the growing marketplace of online storytelling - we encourage you to join with us to shape and build our world.

The web is not just another distribution channel for broadcast signals. It is a conversation between everyone and it is an extension of our nervous systems. We seek to create and share and prosper in this new age of screen culture and interconnected thought.

We are Independent Web Video Creators. (Mills, 2012)

As an act of entrepreneuring in and of itself, the creation of the IWCC solidified many web creators' desires to enact meaningful change for themselves and for others, and to construct a space and market for themselves that reflects their values.

Paying attention to the contextual dimensions undergirding entrepreneuring in this sector, and focusing on the processes of breaking free and breaking up, thus reveal significant insights about the 'who' and the 'why' of web creation in Canada. The practical upshot of this is significant, and will be discussed in detail in the final chapter. 
To be brief, in giving context the attention it warrants, all the while paying attention to agent motives, we come to understand the dynamics of this nascent sector, and then can adjust the focus of entrepreneurial and industrial policy so that it better suits, and responds to, who and why individuals become involved. Policymakers must consider the wide breadth of existing spatial, temporal, institutional and social conditions and scaffolding that exist. In viewing entrepreneurship as a social, rather than merely an economic phenomenon, the urgency for policymakers to tend to wider issues, around racial discrimination, for example, comes to the forefront. At the same time, these findings make a case for a sectoral approach to entrepreneurship/ innovation policy, since such an approach is concerned with entrepreneurial activity in a particular 'system'. Larger, cross-sectoral approaches risk blotting out, blurring or missing pertinent contextual specificities in the search for baseline common denominators. This is problematic because without a meaningful understanding of the various contextual dimensions and constraints, and how they create barriers for entrepreneurial activity in a given system, policy cannot have full effect or impact. It must consider the unique set of structures and challenges faced by those engaging in entrepreneuring in the sector of interest.

Finally, as Rindova et al. (2009) point out, those doing the entrepreneuring "may have only a limited understanding of the solidity of the structures they seek to dislodge" (p.479). As will become clear, many creators, especially the emerging ones, 
lack the skills, understanding, resources and capacity to achieve the change they seek. The next chapter begins to evaluate whether the web has lived up to its promise of more inclusive participation (Benkler, 2006; Burgess and Green, 2009; Jenkins, 2006; Shirky, 2008; Tapscott and Williams, 2007) by examining the composition of key creative teams in Canadian web series. As the following chapter will reveal—despite the motive to both break free from, and to break up the existing status quo by many web creators, and the promise of more democratized access in general, the underrepresentation of women and visible minorities has continued to the Canadian online scripted sector, although there is also room for hope, particularly when the right interventions are set in motion. 
Chapter 5 - Identity and Participation and the Intensified Web Series Model of Production

The underrepresentation of women, visible minorities, and other traditionally marginalized groups both on and behind the screen has been demonstrated across developed, Western, English-speaking nations including Canada, the United States, and the United Kingdom (Creative Skillset, 2015; Hunt, Ramon \& Tran, 2016; WIFT, 2012; WIV, 2015). Scholars examining the issues point to multiple sources of exclusion, including both economic and social dimensions (Allen et al., 2012; Antcliff, Saundry and Stuart, 2007; Grugalis and Stoyanova, 2012; Lee, 2011). This is particularly true for those with intersectional identities, including for example, racialized women, who are affected by barriers not only relating to gender, but to race as well (Crenshaw, 1989).

The absence of an editorial gatekeeper, the decreasing costs of production equipment and the increasing ubiquity of broadband are thought to alleviate some of the sources of exclusion that are commonplace in traditional television (Burgess and Green, 2009; Cunningham and Craig, 2016). Cunningham and Craig (2016) focus on the discontinuities between production for the traditional television and film pipelines, and production for online social media platforms like YouTube, Facebook, SnapChat and others. They emphasize the development of a protoindustry, which they observe demonstrates increased "cultural progressivity" and entails "a rapidly professionalizing and monetizing wave of diverse, multicultural, previously amateur content creators 
from around the world" (Cunningham and Craig, 2016, p.5412). And yet, while this observation regarding increased diversity is likely true in the aggregate, it does not appear to apply evenly across all streams of online content, and as will soon be shown, is only partially true in the case of scripted web series.

As was outlined in Chapter 3, gender and race were recorded and analyzed across the categories of writer, director and cinematographer for both IPF and selffunded Canadian web series distributed between 2010 and $2015 .{ }^{5}$ To recapitulate, the inclusion criteria were series that were English-language, narrative, live-action, consisting of three episodes or more, distributed first over the internet and aimed at youth or adult audiences. After removing series from the IPF's funding repertoire that did not fit these criteria, 36 series remained. A total of 139 self-funded web series that met the criteria and were independently produced (without broadcaster affiliation or support from a media grant or fund) were identified. Out of the 139, complete credits were collected for 137 of these series. These two series for which various credits were missing were included in the analysis only when the missing information would not skew outcomes, for instance, in analyses of geographical origin. The researcher hypothesized that web series with IPF funding would display less diversity in terms of gender and race than the web series produced without such funding, but that both

\footnotetext{
${ }^{5}$ One series, "YidLife Crisis", received the "Shaping our Future" grant from a Jewish community organization. Since this was not a grant specific to web series, or even media more broadly, but open to anyone of that faith, the researcher included the series in the analysis.
} 
groups of web series would exhibit more diversity in creative teams than in regular television and film. Application to a funding program takes a certain level of institutionalized knowledge and understanding. The IPF, however, at least at the time that these web series were produced, contained a less onerous application process, when compared with the criteria required for traditional television funding, although it did require the filming of a short 'proof of concept' video. Importantly, however, the IPF did not require broadcaster commitments, unlike funding programs for traditional television. The chapter begins with the presentation of the results of the diversity analysis. These results are then contextualized and explained through an examination of the general creative industries model of production (Eikhoff and Warhurst, 2013), which is subsequently shown to be more intensified in web series. The intensified model is then linked to the persistence and perpetuation of the underrepresentation of women and visible minorities in web series.

\section{i. Gender}

Figure 1 and Figure 2 below represent the gender distribution in writer, director and cinematography categories across IPF and self-funded web series, respectively. 
Figure 1

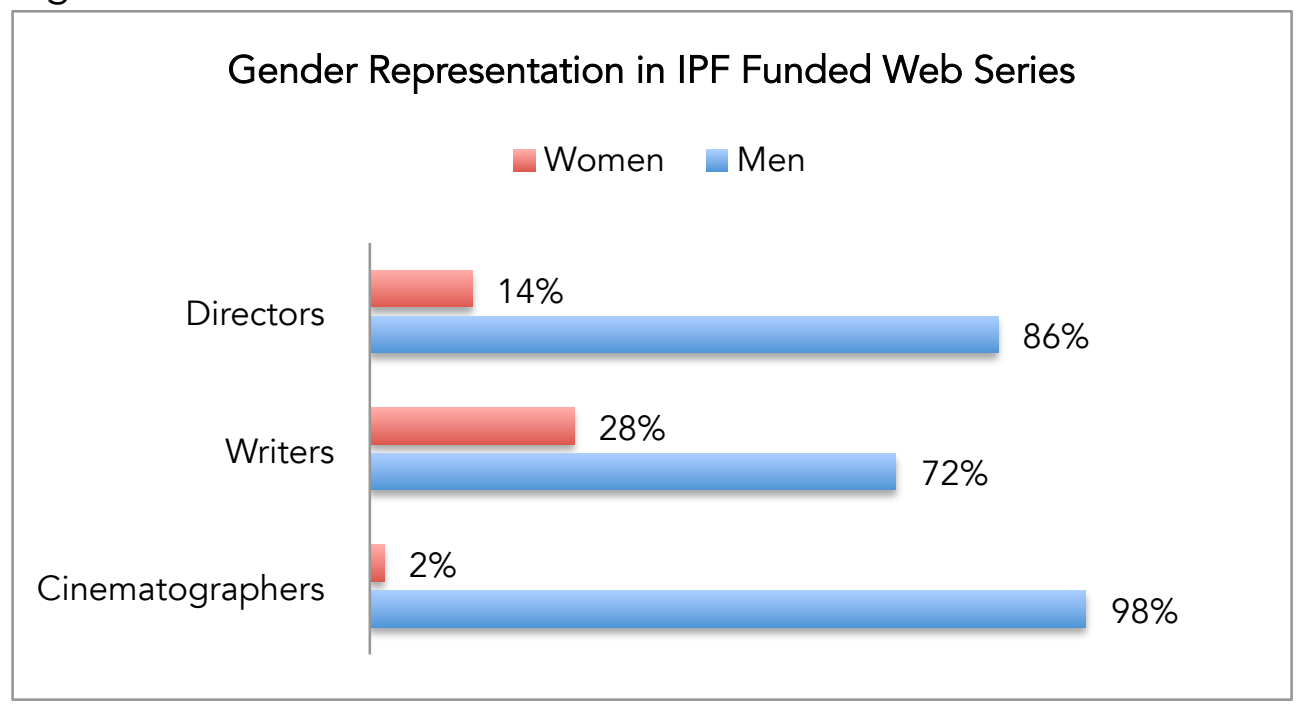

Figure 2

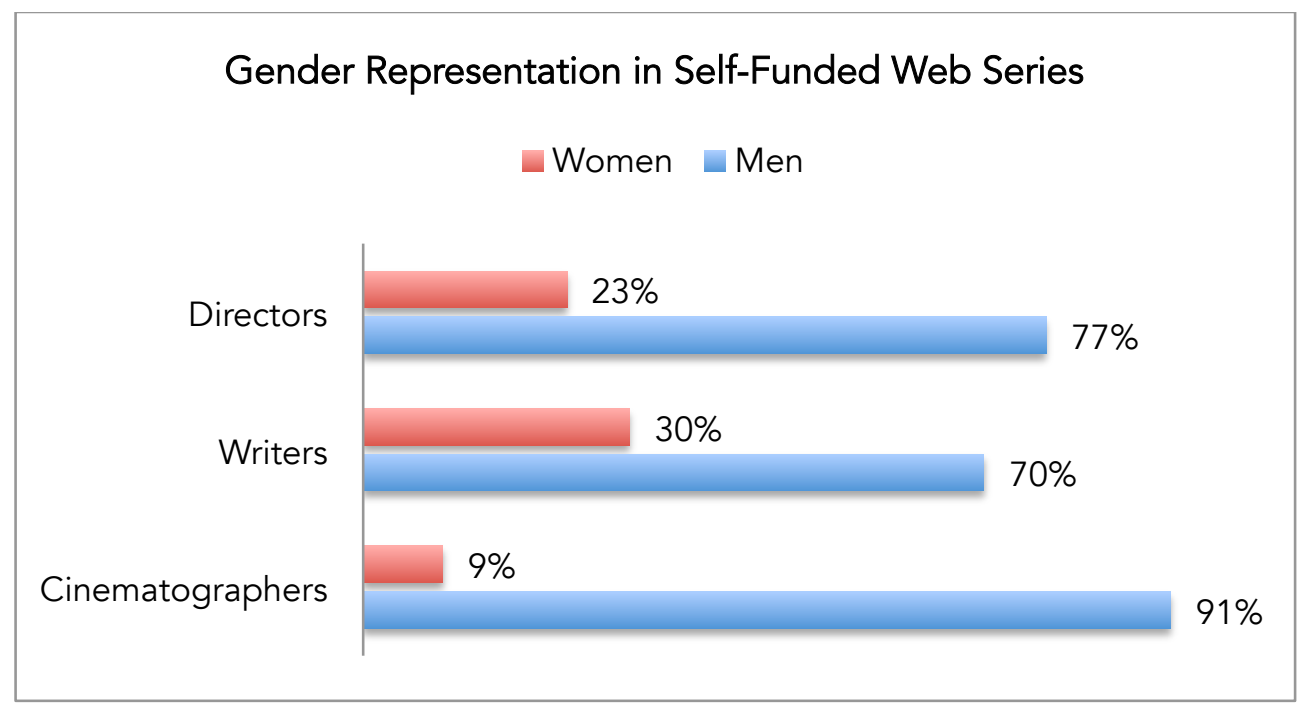

As is clear from both of the above figures, significant underrepresentation of women, who represent half of the general population, and nearly half the general workforce (ESDC, 2015), remains in the online space. In 2014, women reached a Canada-wide workforce representation rate of $41 \%$ in the federally regulated private sector (which 
includes broadcasters) (ESDC, 2015). As was expected, women were better represented among web series produced with no support from formal media funding or grant programs than in the series that received IPF funding. Figure 3 below compares the levels of gender representation in the key roles of director, writer and cinematographer in the web series analyzed when compared to the distribution of gender in television and film. It is difficult to determine which set of data (TV or film) is the more accurate point of comparison. Because of broadcaster involvement in television, there is a more established pipeline in the development of television, than in film. In this sense, films are more 'entrepreneurial' than television productions and are most like independent web series, which are often one-offs, and if they contain multiple seasons, especially in the case of self-funded web series, each season is refinanced again from the start. There are also fewer 'checks and balances' in the film and web series worlds than in television because of television's more institutionalized status. Web series, however, are more like television in the sense that they are serialized. The collected data are thus presented in several ways below. 
Figure 3

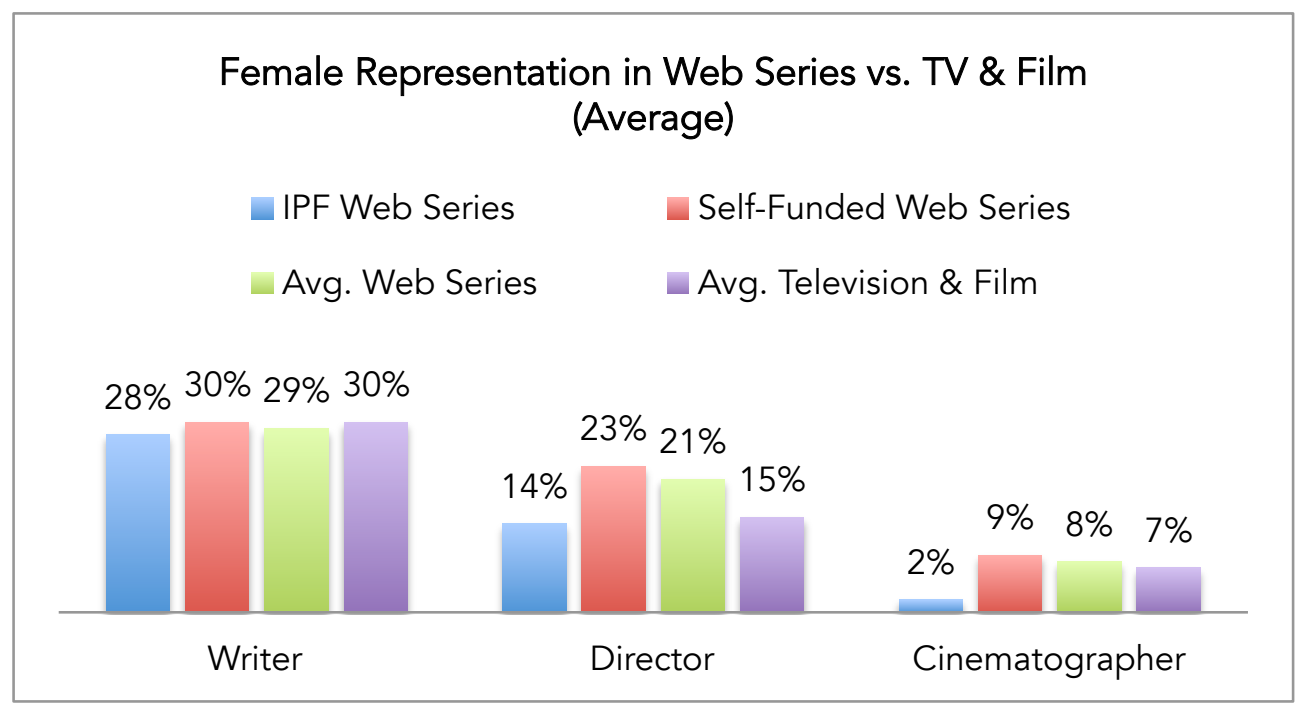

*Television and film statistics averaged from WIV $(2012 ; 2013 ; 2014 ; 2015)$ reports. ${ }^{6}$

From Figure 3, it is clear that self-funded web series appear to provide significant inroads to women in the director category, which is, according to many, the primary creative decision-making role in production. There are typically also fewer director positions than writer positions in screen productions, making an increase in this category worthy of note. In self-funded web series, women held nearly $23 \%$ of directing roles, compared with just $15 \%$ in television and film. The representation of women directors in IPF web series is nearly identical, at $14 \%$, to the traditional industry (15\%). It is important to note that both the film and television figures stem from projects receiving government funding, and requiring significant experience and

\footnotetext{
${ }^{6}$ These reports together represent film and television projects tracked between and including 2010, up to and including the fiscal 2013-2014 year.
} 
credentials, as well as partnerships with distributors and broadcasters. Figure 3 shows nearly no difference in the writer role between web series receiving IPF funding, those made without media program grants or funds, or the average representation levels in film and television. In the cinematography role, again, when averaged, web series showed only a $1 \%$ overall improvement in participation rates of women over film and television. When disaggregated, it becomes clear that the greatest gain was seen in the self-funded web series category. There was a significant difference between the low representation of women in IPF funded web series in the cinematography role (at $2 \%$ ), and the rest of the categories, which ranged from 7\% (TV and film) to 9\% (selffunded web series).

When the television and film figures are disaggregated into separate categories, an interesting pattern emerges in the cinematography role. There are, however, no significant or interesting patterns in the director and writer roles that are not already demonstrated in Figure 3. 
Figure 4

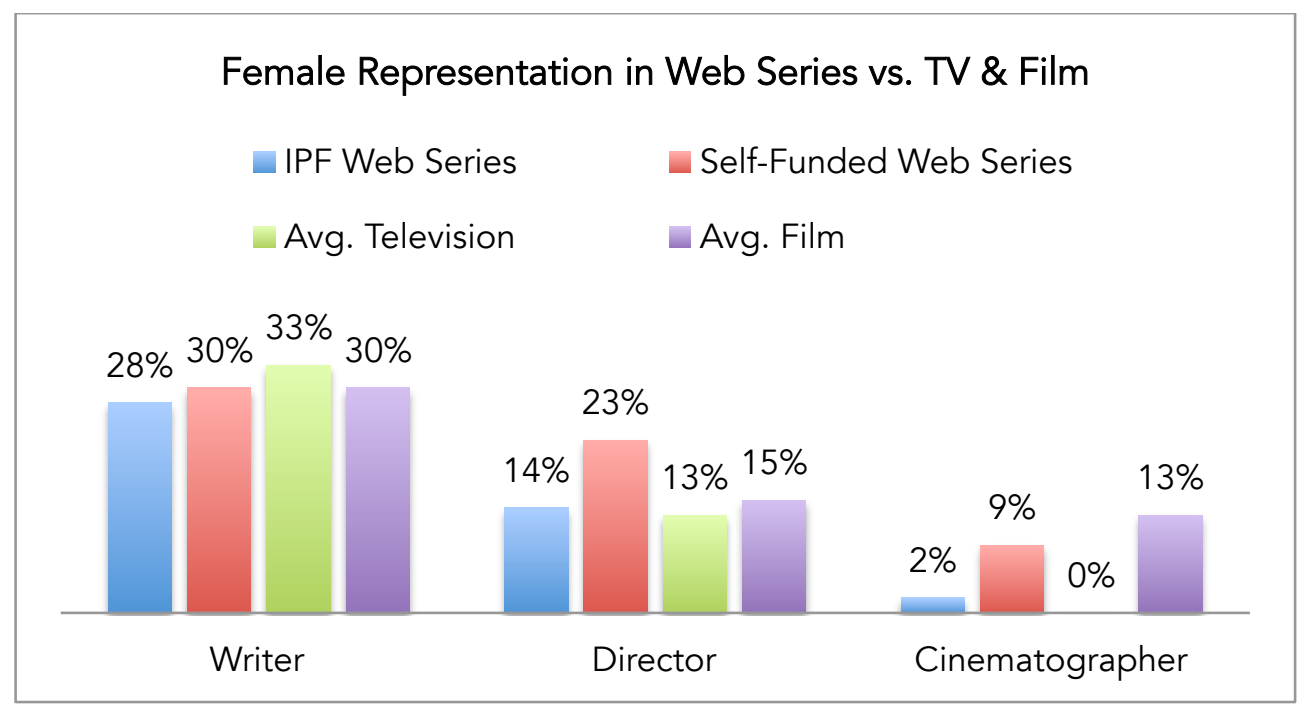

*Television and film statistics averaged from WIV $(2012 ; 2013 ; 2014 ; 2015)$ reports. $^{7}$

From Figure 4, it becomes clear that the representation of women in writing positions holds nearly the same (between $28 \%$ and $30 \%$ ) across funded and self-funded web series, and the separate television (33\%) and film (30\%) categories. In the director role, again the only significant variation in the representation of women occurs in the self-funded web series category where women hold $23 \%$ of the director credits. Representation of women in directing roles is nearly the same across IPF web series (14\%), and the average television (13\%) and film (15\%) figures. In the cinematography category, women were the least represented in IPF funded web series at $2 \%$ and in

\footnotetext{
7 These reports together represent film and television projects tracked between and including 2010, up to and including the fiscal 2013-2014 year.
} 
television at $0 \%$ (there were no women across 75 aggregated cinematography credits in television). ${ }^{8}$ Film demonstrates the highest share of female cinematographers (13\%), followed by self-funded web series (9\%). It appears that the more entrepreneurial and less structured pathways presented by self-funded web series, and feature films offer the most opportunity for women to move into cinematography positions.

A deeper look at the cinematography category for both categories of web series is revealing. In 20 out of 22 instances (91\%) where women are cinematographers in self-funded web series, a woman is also part of the directing or producing team. Of 61 self-funded web series that had an all-male producer and director team, 97\% did not engage a female cinematographer, while only $3 \%$ did. Of the 62 self-funded web series that had an all male producing team, only $3 \%$ engaged a female cinematographer. In $90 \%$ (19 of 21 ) of self-funded web series in which a woman is a cinematographer, a woman is on the producing team as well. Again, these numbers do not suggest that women are more likely to hire women cinematographers in general over men, but only that when a woman is a cinematographer, it is more likely that there are also women on the producer and directing team. It appears however, that all-male producer and/or director teams are not as likely to hire a female cinematographer than teams in which genders are mixed.

${ }^{8}($ WIV, 2015) 
When examining the producer category, $46 \%$ (63/138) of self-funded web series, did not engage a woman in a producer capacity. Of the series that did not have a woman engaged as a producer, $98 \%$ also did not engage a woman in a writer or director role. Of the self-funded web series in which there was a woman as part of the producing team, $73 \%$ engaged a woman in a writer or director role. This suggests a gendered relationship between the producer and writer and director roles. It appears that when a woman is part of the core producer team, she is more likely to engage women in other key creative roles than when there is an all-male producer team. The same pattern emerged in IPF funded web series where $50 \%$ of series did not engage a single woman as writer or director. Of the $50 \%$ that did, only six percent contained an all-male producing team. When there was a woman present as part of the producing team, a woman was engaged in a writer or director category $47 \%$ of the time.

\section{ii. Race}

In 2014, visible minorities reached a Canada-wide workforce representation rate of $20 \%$, which is higher than their labour market availability (LMA) of just under $18 \%$ (ESDC, 2015). This suggests that overall, there has been progress in the representation of visible minorities in the Canadian workforce. In fact, visible minorities were the only designated group (women, visible minorities, persons with a disability, and 
Aboriginals') to surpass their LMA (ESDC, 2015). Figure 5 below demonstrates the rates of visible minority participation in both IPF funded and self-funded web series.

Figure 5

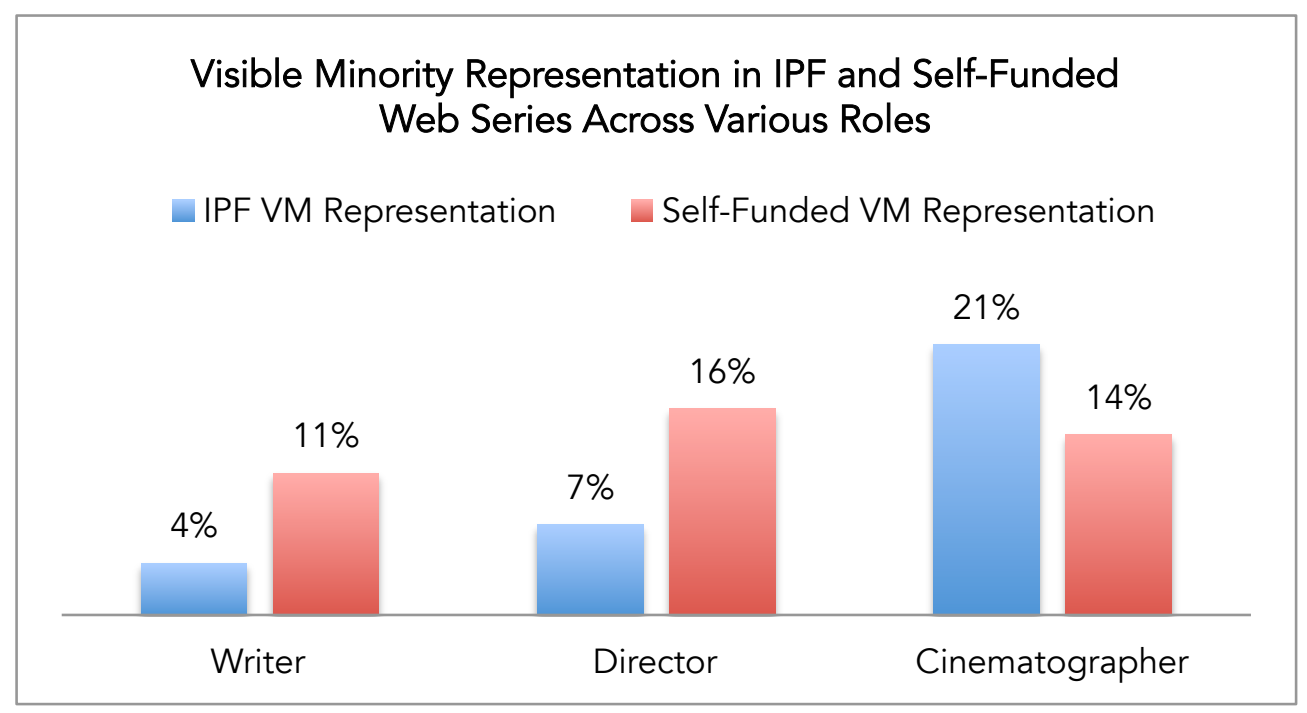

Just as is the case in traditional film and television (Coutanche \& Davis, 2013; Davis, Shtern, Coutanche, \& Godo, 2014; Kaye \& Davis, 2011; WIFT, 2012), visible minorities continue to be underrepresented in the key roles of writer and director, although again, self-funded web series appear to provide significant inroads toward their participation in these categories. According to a firm-level survey of the Canadian

\footnotetext{
9 The term "Aboriginal" is utilized throughout this dissertation to remain consistent with the terminology employed by Statistics Canada (2015), the Broadcasting Act (1991), and the Employment Equity Act (1995). It is acknowledged, however, that "Indigenous" is the more commonly utilized and preferred terminology today.
} 
broadcasting industry conducted in 2012, which represented nearly 27000 workers, WIFT (2012) found the representation rate of visible minorities to be seven percent. Visible minorities were most represented in roles that required specialized skills including business affairs, legal, finance, accounting, IT and technical engineering (WIFT, 2012). They were most poorly represented in key creative decision making roles including original production and development (WIFT, 2012). In their study of Canadian screenwriters, Davis, Shtern, Coutanche and Godo (2014) found that just four percent of Canadian screenwriters are visible minorities.

Although there has not been any systematic or longitudinal tracking of visible minorities in television or film director roles in Canada, their general representation in the broadcasting workforce, and their underrepresentation in original production and development as determined by WIFT (2012), in addition to Canadian anecdotal evidence, as well as quantitative evidence demonstrating marked underrepresentation from the United States (Hunt, Ramon \& Tran, 2016), suggest that their underrepresentation in this role would be pronounced.

Figure 5 thus demonstrates that in the key creative decision making categories of director and writer, visible minorities were significantly better represented in selffunded web series, than in IPF web series, suggesting that the funding mechanism presents a barrier to their participation. Another way to interpret this data, which adds to, rather than contradicts the previous observation, is that visible minorities decidedly 
fare worse in more 'professionalized' or institutionalized systems where financing, network connections and greater accountability are required and involved. While not all self-funded web series can be said to be 'amateur' or 'semi-amateur' (some are decidedly 'professional') with respect to financing, quality and production organization-many are. As was revealed in the interviews, many web series creators engage to create a first calling card piece that they hope could ameliorate their status and chances of success.

The trend of increased visible minority participation in self-funded web series is reversed in the cinematographer role, where IPF web series contain a larger share of visible minority cinematographers than do self-funded web series. This is an interesting finding. The proportion of participation of visible minorities in IPF series at $21 \%$ is higher than their participation in any other category for both self-funded and IPF series, and the only category in which visible minorities surpass their LMA of $18 \%$. It would appear then that at the more professionalized level—visible minorities fare worse in the non (strictly) creative roles. The role of a cinematographer is a hybrid role, requiring not only creative vision, but highly specialized technical skills—a trained craft. This appears to be consistent with the findings of WIFT (2012) which, again, found visible minorities to be the least represented in key creative roles such as original program development, and most represented in roles that require specialized education (ex. law; accounting) or technical training and understanding (IT and technical engineering). Neither the 
American Society of Cinematographers (ASC), nor the Canadian Society of

Cinematographers (CSC) publicly disclose information on the diversity of their membership, so it is not possible to definitively say whether visible minorities are better represented in web series than they are in the traditional screen industries. The more interesting observation to be made here is the difference in representation when moving from a less professionalized arena (self-funded web series) to the more professionalized IPF series.

An interesting insight comes from examining the distribution of visible minorities across the three key categories of interest (writer, director and cinematographer). A total of 20 IPF web series, or $56 \%$, had no visible minority person in any of these categories. Of the 16 or $44 \%$ that did, 10 or $63 \%$ had a person of colour in a cinematographer position only. At the self-funded web series level, this trend was reversed: Of the self-funded web series which contained a visible minority person in a writer, director, or cinematography role, $76 \%$ had persons of colour in a writer or director position. This means that visible minorities were present in the cinematography position only in just $24 \%$ of these series. These numbers add further strength to the picture that emerges in Figure 5 above. At this less professionalized level, more visible minorities are able to engage in the key creative roles of writer and director than at the IPF level. 
Examining the relationships between the various categories is also revealing. Of the 36 IPF funded web series, 31 or $86 \%$, did not engage a single visible minority person in a writer or director role. Of the five that did, $60 \%$ had a producer who was also a visible minority. Objectively, it is important to keep in mind that the population of IPF series is small. The larger population of self-funded web series, however, confirms this pattern: Out of 138 series for which writer and director credits are known, 101 or $73 \%$, engaged no visible minority in a writing or directing role. Of the 37 series in which visible minorities are engaged as writers or directors, $70 \%$, also have a visible minority person engaged in a producer capacity. Examined another way, 95 of 138 self-funded web series, or $69 \%$, did not have a visible minority as part of the producer team. Of these 95 series that did not engage a visible minority in a producer capacity, $90 \%$ also did not engage a single visible minority person as a director or writer. There does, therefore, appear to be a relationship between visible minority presence in the producer team and the engagement of visible minorities in the key creative writing and directing roles.

iii. Gender and Race Another important set of insights are revealed when examining the cross section of gender and race. Figure 6 and 7 below demonstrate the proportion of visible and nonvisible minority women, and visible and non-visible minority men in IPF funded and self-funded web series. 
Figure 6

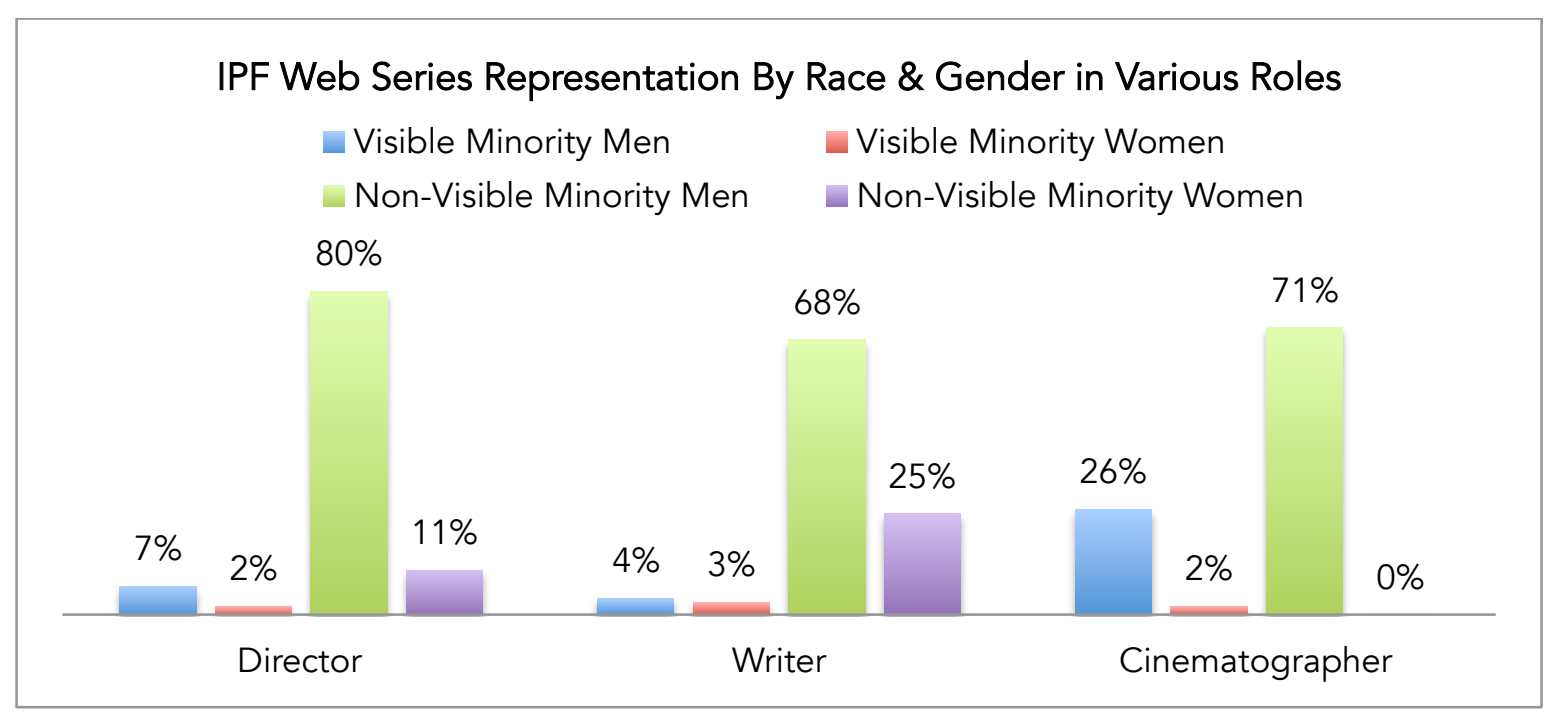

Figure 7

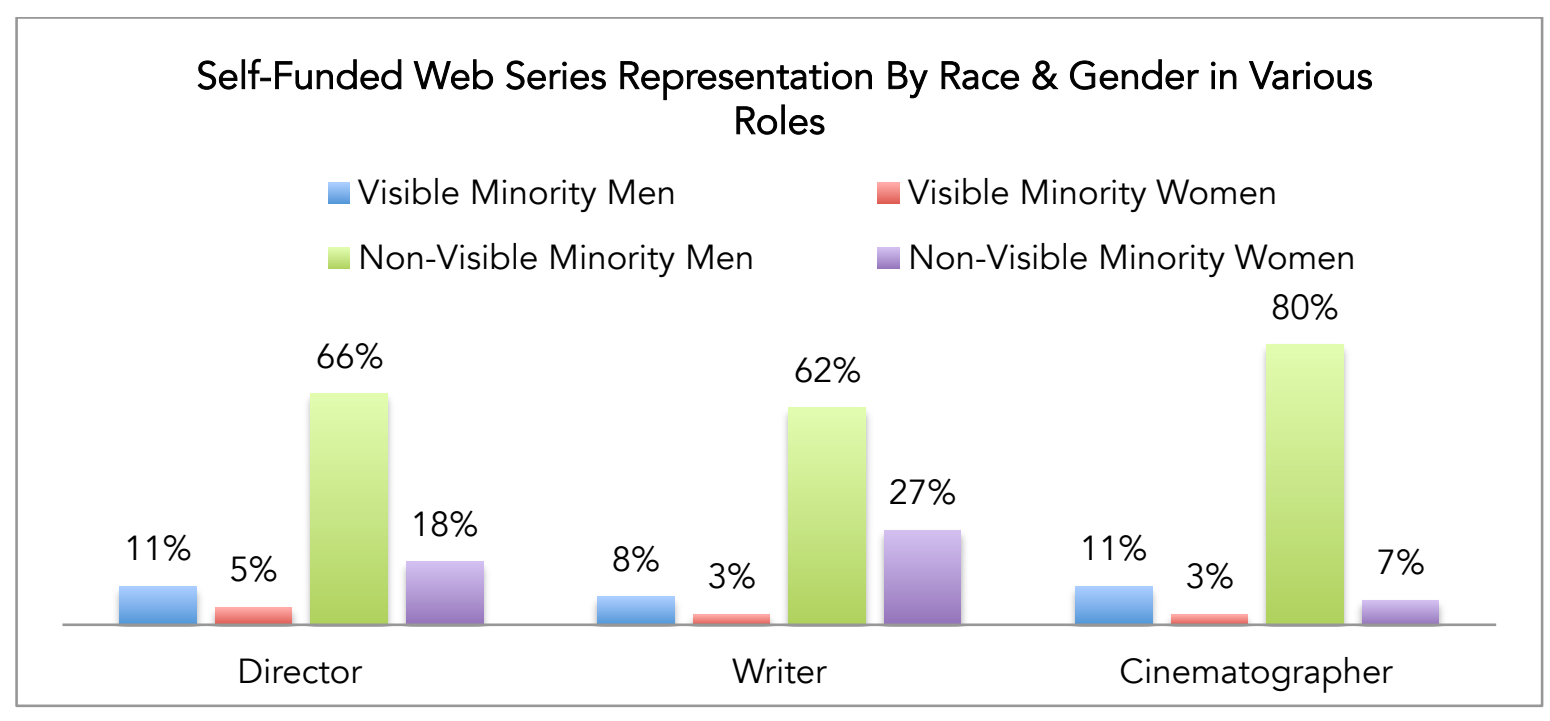

Non-visible minority men are the most highly represented group across all roles. Visible minority women are consistently the least represented segments in key creative roles in both sets of web series. Although no studies consistently follow the intersection of race and gender in television in a robust way, WIV $(2012 ; 2013)$ did find that racialized and 
Aboriginal women were the least represented cohort in Canadian television in each category with representation rates ranging from 0-1.5\%. Figures 8 to 10 demonstrate the share of credits in IPF web series that are held by visible minorities in each category when broken down by gender.

Figure 8

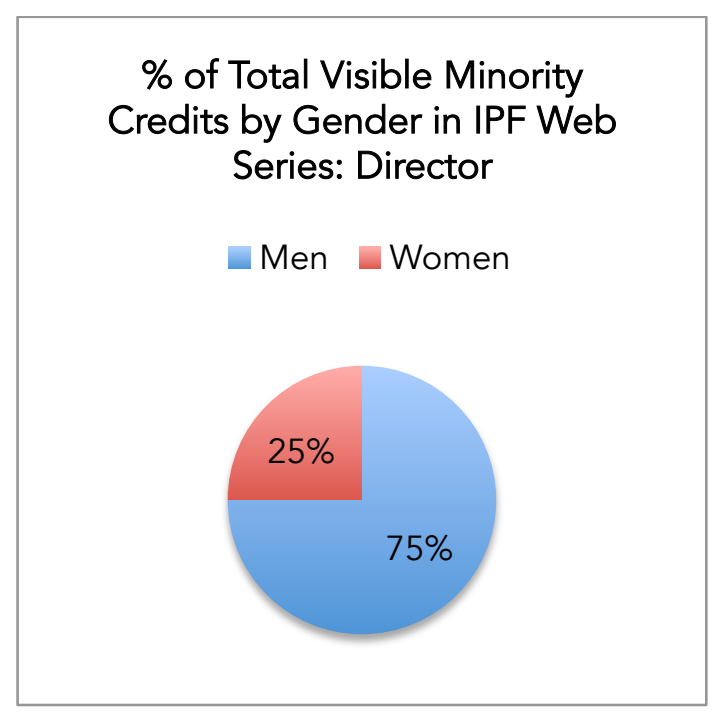

Figure 9

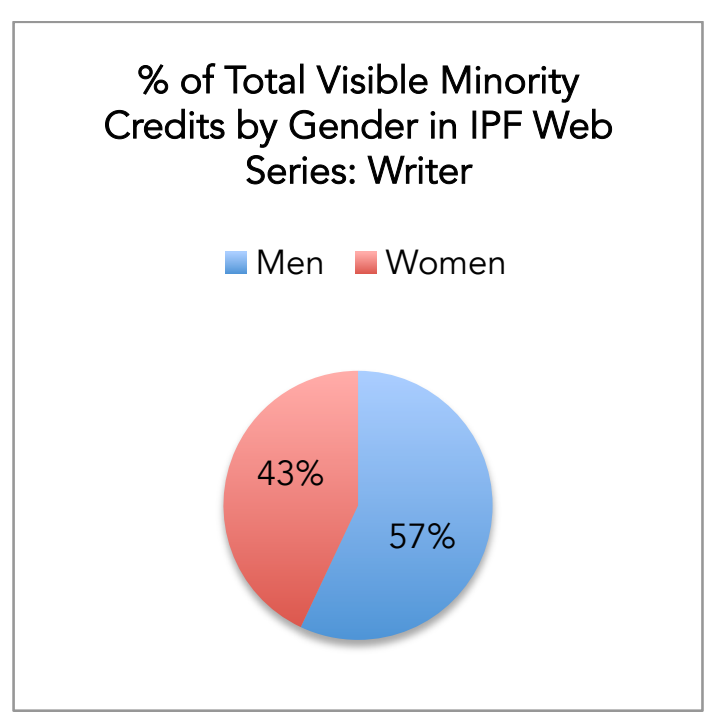


Figure 10

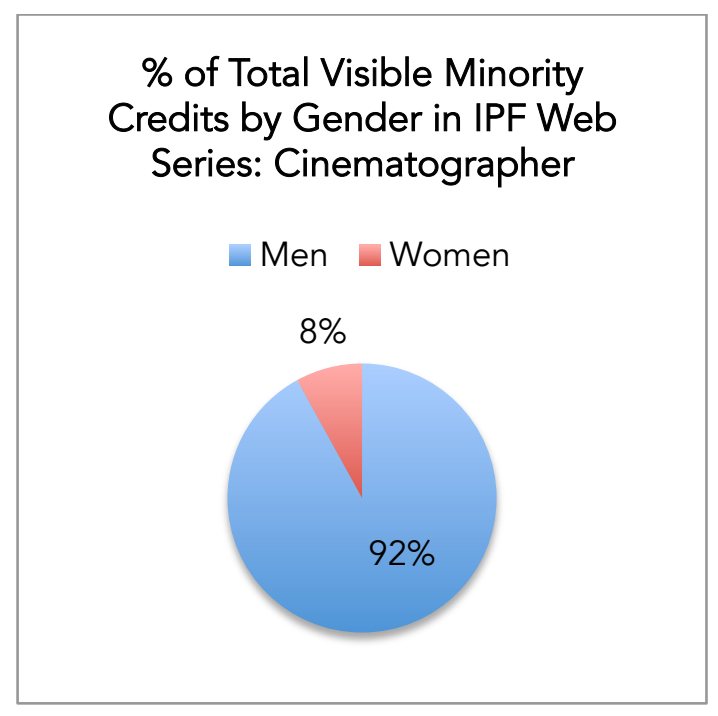

Even though there is underrepresentation of visible minorities across director and writer categories in IPF series, in an ideal (all things being equal) scenario, these credits would be split evenly between visible minority women and men. The division is not equal and the gap is the most marked in the cinematography category, which is consistent with television and film statistics as demonstrated in Figures 3 and 4 previously. For every one female visible minority director, there are three male visible minority directors. This is a significant disparity. Visible minority women fared better as a share of the total visible minority credits in the writing category, holding $43 \%$ of the credits. Although still underrepresented, as was shown in Figures 3 and 4, women fare better as writers in film and television than as directors. 
Figures 11 to 13 demonstrate the share of credits in self-funded web series held by all visible minorities in the three categories of interest when broken down by gender.

Figure 11

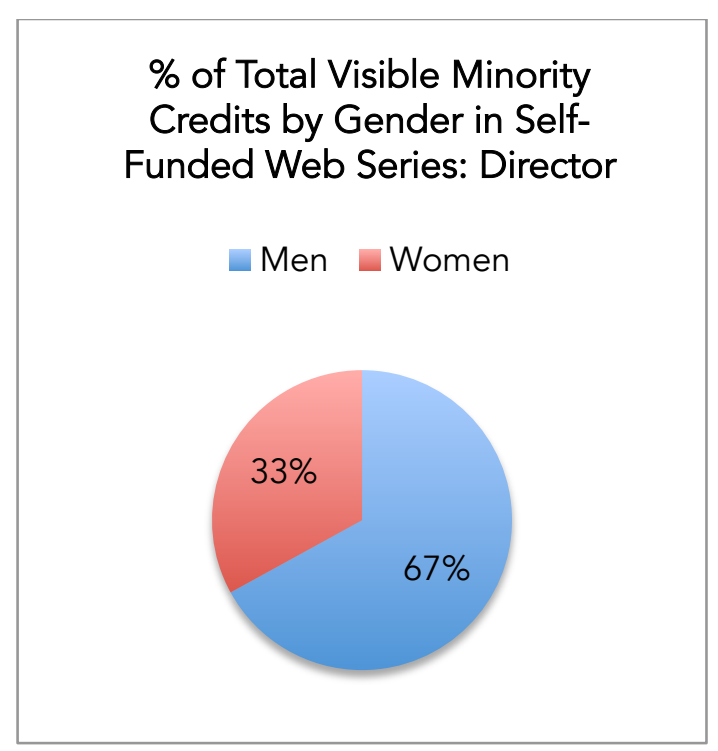

Figure 12

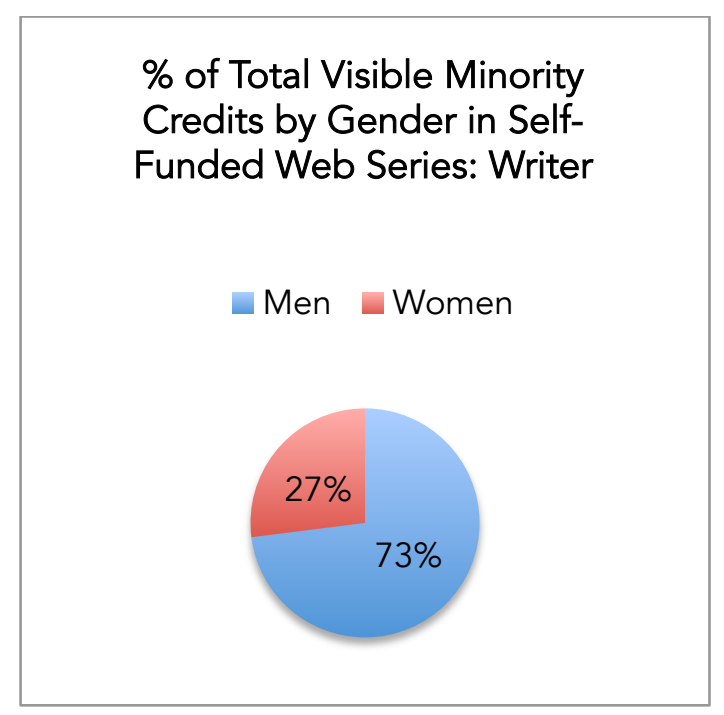


Figure 13

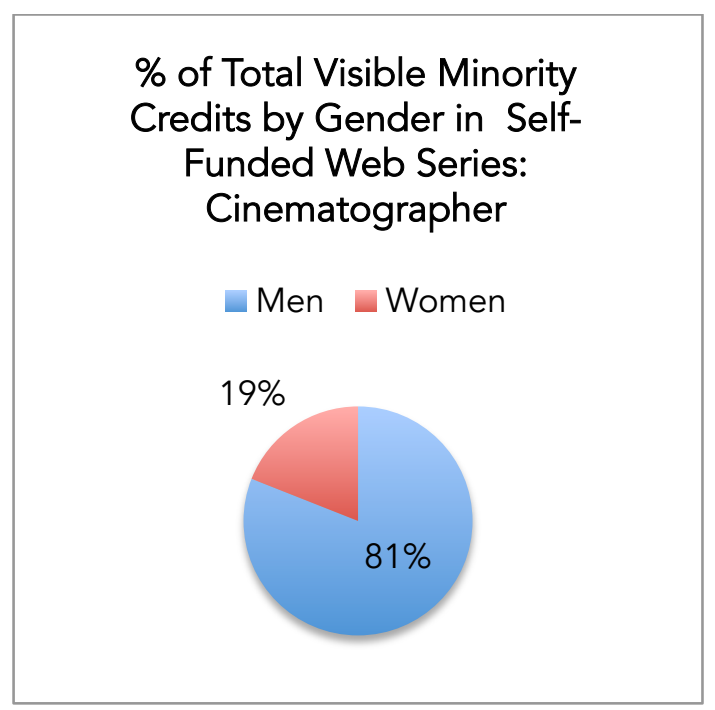

The proportions of total visible minority credits held by women across the various categories are different here than in the IPF web series. Here, a larger proportion (33\% compared to $25 \%$ ) of total visible minority credits in the director category are held by women. In the writing category, there is a significantly smaller proportion of credits held by visible minority women than in the IPF web series. A much larger share of visible minority women hold credits in the cinematography category as well (19\% compared to $8 \%$ ). It appears then that the proportion of visible minority women fluctuates according to the representation of women in these categories in general. For all women in general, more hold the higher control position of director in self-funded web series than IPF series; fewer, in contrast, are writers; a greater number of women are cinematographers in self-funded web series. The more open, less institutionalized/ 
professionalized web series thus provide visible minority women with an opportunity to more meaningfully participate in roles that provide more 'control' (director), or are more 'unconventional' (cinematography).

iv. Regional Representation

Figures 14 and 15 demonstrate the provincial affiliation of both IPF web series, and self-funded web series, respectively.

Figure 14

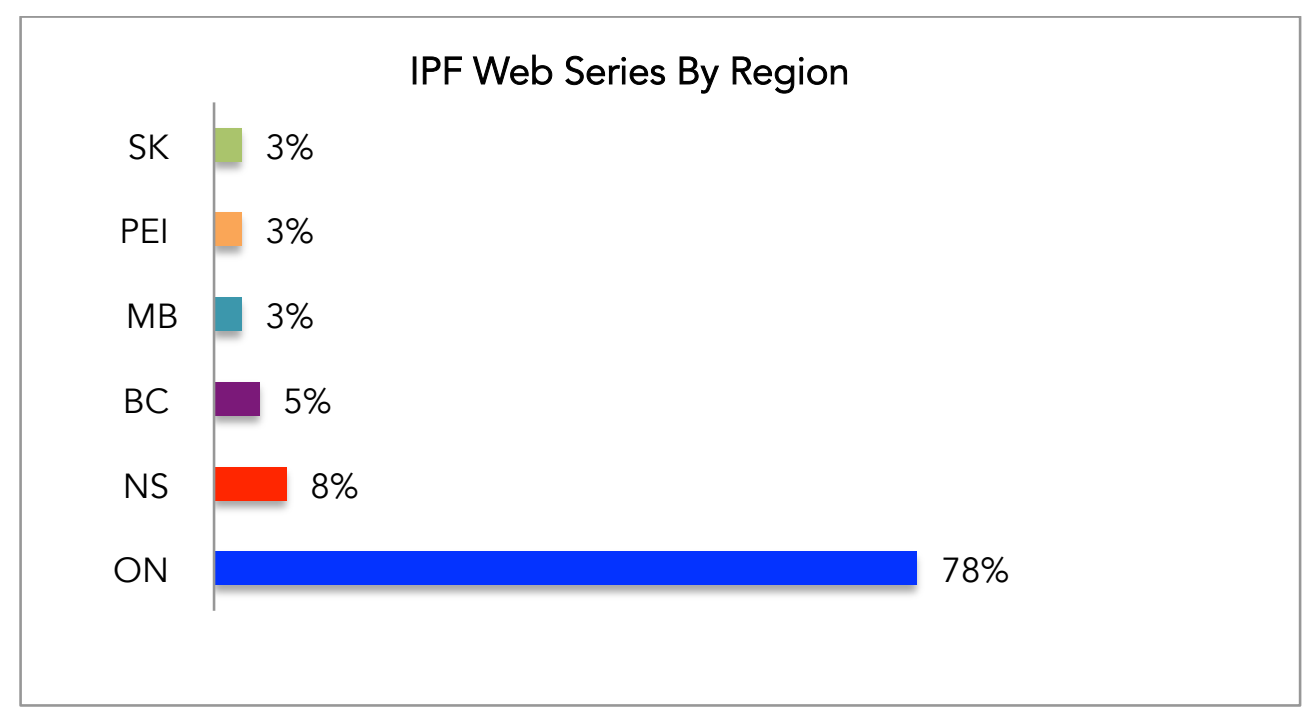


Figure 15

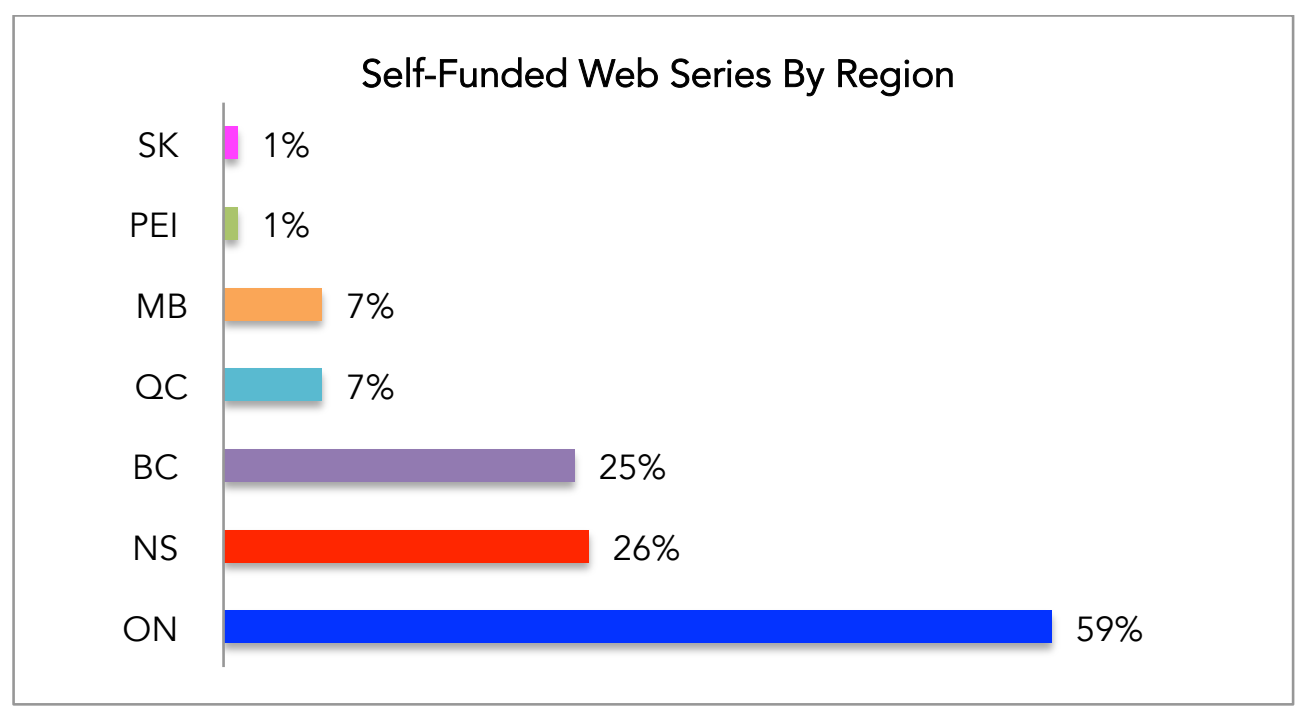

The largest share of both IPF and self-funded web series are Ontario-based. And while this is true, the share of web series from Ontario is higher in IPF series (at 78\%) than in self-funded web series (at 59\%). Ontario is therefore home to a greater proportion of professionalized web series activity, while self-funded web series provide an avenue for increased participation and experimentation from creators in non-Ontario provinces. Interestingly, although the proportion of participation changes from the IPF web series to the self-funded web series significantly, the provinces generally follow the same overall order..$^{10}$ Ontario is the most represented province, followed by Nova Scotia, then BC, with PEI and Saskatchewan tied for last place. In the case of IPF web series, Manitoba is in last place with PEI and Saskatchewan. In self-funded web series, Manitoba is tied with Quebec for fourth place. The greatest variation between the two

\footnotetext{
10 There were no English-language web series based in Quebec in the 2010-2015 IPF web series population.
} 
types of web series occurs in relation to the share of web series from Nova Scotia and British Columbia. These provinces possess a much greater share of web series in the self-funded population (Nova Scotia at 26\%, and BC at 25\%) than in the IPF population (Nova Scotia at $8 \%$, and British Columbia at $5 \%$ ).

Interestingly, the ordering is significantly different from the distribution of traditional television and film production across Canada. According to the CMPA (2016), Ontario held the greatest share of total production at 43\%, followed by Quebec at 33\%, British Columbia at 17\%, Alberta at 3\%, Manitoba and Nova Scotia and Newfoundland each at 1\%, New Brunswick and Saskatchewan at less than 1\%, and PEI at $0 \%$. While in traditional film and television Nova Scotia is a marginal player, in the world of web series it is the second most represented province in both IPF and selffunded web series.

\section{v. Regional Representation and Race: Ontario}

Another important layer of information is revealed when examining race in relation to regional representation levels. The Canadian LMA of $18 \%$ is an estimate of labour market availability for the country, but examining representation rates regionally provides an even more attuned measuring stick. Figure 16 demonstrates visible minority participation levels in both IPF and self-funded web series across writer, director and cinematography roles when compared to the population-wide Ontario 
and Toronto visible minority representation levels for these regions. Ontario-based web series were chosen to be examined more deeply in this way, over the other provinces, due to the high level of visible minority representation in the province, and since the majority of web series are based in Ontario.

Figure 16

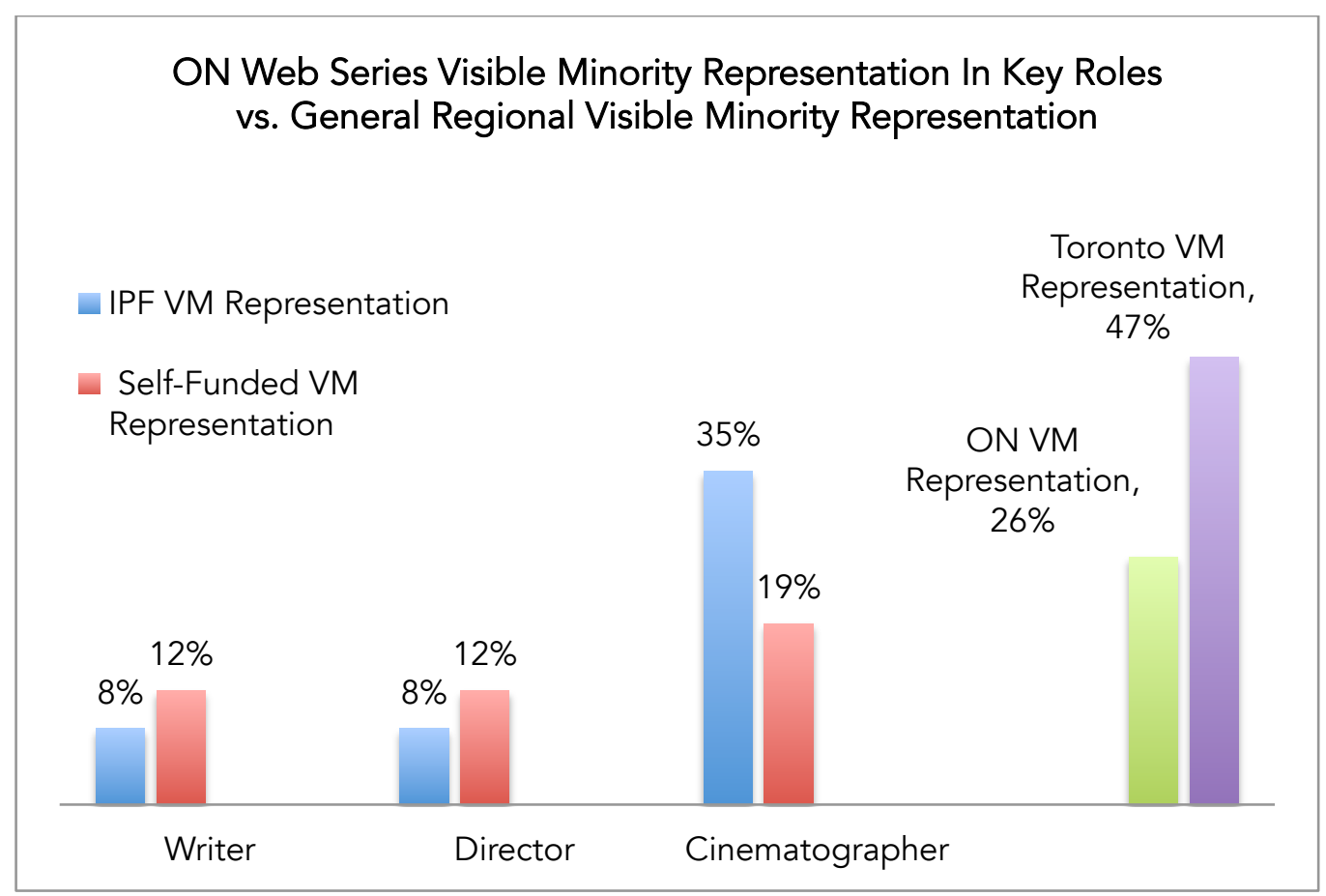

${ }^{*} \mathrm{ON}$ representation rate from Statistics Canada (2013)

*Toronto representation rate from Toronto (2017)

When examining the visible minority representation rates in both IPF and self-funded web series across the three categories, against the provincial representation rate of $26 \%$, it is clear that there is significant underrepresentation except in the IPF web series cinematography category. 
Of the 28 Ontario based IPF web series, 27 or $96 \%$ can be confirmed to be Toronto-based, with the remaining web series being from the Greater Toronto Area (GTA). The more accurate comparator for these web series then is the figure for Toronto-based visible minority representation, which is $47 \%$. With this as a measure, the underrepresentation of visible minorities in IPF web series is even more pronounced. When compared to the Toronto figure, visible minorities are even underrepresented in the cinematography category. Visible minorities comprise $38 \%$ of GTA residents. This means that even when compared to this figure the rates of participation of visible minorities in IPF web series is low.

The same holds true for self-funded web series. Of 83 Ontario-based selffunded web series, 75 or $90 \%$ are from Toronto. This means that the more relevant point of comparison for these series is the Toronto-based representation figure of $47 \%$. Self-funded web series do not come close to this level of representation.

\section{Discussion and Analysis}

Although self-funded web series do appear to provide significant inroads to the participation of women and visible minorities in certain roles when compared to film and television, both groups are still underrepresented. This is especially true in web series receiving funding. All of the web series examined in this portion of the study (including the IPF series), aside from a nominal few, were released on YouTube. The 
findings thus importantly refine the scope of the observation surrounding the increased presence of diversity on social media platforms such as YouTube (Cunningham and Craig, 2016), and emphasize the importance of examining unique web content streams separately.

To reiterate, Cunningham, Craig and Silver (2016) make the important distinction that the new pure-play internet companies like YouTube and Facebook operate according to a very different logic than do Hollywood-based firms. According to the authors, the pure-play internet companies operate according to a shared NoCal (Northern California, Silicon Valley) logic that values "scale, automation, permanent beta, rapid prototyping and iteration" (Cunningham, Craig and Silver, 2016, p.4). The Hollywood firms, in contrast, operate according to a shared SoCal (Southern California) logic, which holds as important the time-honoured, "core Hollywood skills of acting, screenwriting or directing", among other things (Cunningham, Craig and Silver, 2016, p.10).

While Cunningham, Craig and Silver (2016) recognize that YouTube's history since its purchase by Google is "a history of Google seeking to come to terms with the SoCal fundamentals of entertainment, and content and talent development, from its NoCal base as an IT company..." (Cunningham, Craig and Silver, 2016, p.4), their observation of these duelling logics can be further extended to the micro and mid levels to add to the conceptualization and understanding of unique web content 
streams and their operations. It is thus not only the platforms, but also the various creators and firms that populate them with their work that contain both NoCal and SoCal mindsets and logics. The adoption of one logic over another, or indeed, the hybridization of these logics, affects creators' chances of success on platforms such as YouTube.

Cunningham and Craig (2016), for example, argue that, Across these on-demand, ephemeral, and live broadcasting platforms, creator entrepreneurs engage in content innovation in contrast to traditional entertainment, without little interest in developing original intellectual property or building content libraries. This new screen ecology is driven by intrinsically interactive, viewer- and audience-centricity. (p.5413)

While this might be true for those who embrace the NoCal logic, scripted web series creators, active users of the same platforms, pursue a SoCal logic that is more closely connected with Hollywood and holds as important the core Hollywood skills. As was made clear in the previous section-many web series creators engage in online production because it is seen as an entry point into the traditional system, a system, which for many, based on the findings of the interviews, is still the epitome of professionalism and mastery. These creators aim to produce TV-like content for the web. One creator reflected on how he crafted his web series to resemble television, and how he hoped others would see it that way: "The big thing was for somebody to watch it and put it among other shows in their list of things that they like-not to 
consider it its own category, but to see it as a [TV] show" (Jeremy Larter, Independent creator).

While the mismatch between the SoCal web series format, and the largely NoCal YouTube platform, and its effects on web series' chances of success will be explored in more depth in Chapter 6, the observation that the segment of creators of central concern in this study-creators of scripted, narrative web series, often adhere to, and indeed strive toward, certain key SoCal values and aesthetics, helps explain why there is not greater diversity amongst the population.

Because scripted web series typically contain similar 'building blocks' to television and film (script; production team most often involving several members including producer, writer, director, cinematographer, editor; cast often involving at least greater than one actor; sets and props) the model of production is also similar. Scholars Eikhof and Warhurst (2013) argue that it is the project-based model of production typical of most creative industries (which include architecture and design; film, television, video, radio and publishing; fine arts; music and the performing arts; software and computer gaming; advertising and crafts) that contains systemic and persistent social inequalities. Synthesizing previous research on work in the creative industries, the authors argue that the model of production affects four major aspects of work and employment in this sector (Eikhof and Warhurst, 2013):

1. The organization of work and employment 
2. Training and entry into the field

3. Recruitment practices

4. Working patterns

In the section that follows, Eikhof and Warhurst (2013)'s breakdown of how the production model affects the four areas of work in the creative industries outlined above will be filled in with other relevant and supporting literature. Wherever possible, research specifically on the impact of the model of production on work in film and television will be cited.

General Creative Industries Model of Production

To begin, according to Eikhof and Warhurst (2013), the creative industries model of production affects the organization of work as it induces an environment that is intensely insecure. Work that is based around a life-of-project model results in shortterm contracts where individuals with diverse skill sets come together for the duration of a project, and typically disband once it is complete (Caves, 2000; Eikhof and Warhurst, 2013; Gill and Pratt, 2008). Under these temporary conditions, workers often must bear the cost of all social and economic risks including unemployment, childcare, and health (Eikhof and Warhurst, 2013). A recent study of Canadian television workers revealed that $66 \%$ of the study's respondents identified as freelancers and only $30 \%$ were employees (CMG, 2013b). In their evaluation of Canadian labour policy, Gollmitzer and Murray (2009) found that although recent changes to Canadian 
employment law permit self-employed workers to access limited aspects of the Employment Insurance program, including the possibility of receiving parental, compassionate care and sickness benefits, many creative workers are ineligible to enrol due to "the draconian hours of work requirements" (p.431). Under the current legislation, no self-employed person is eligible to receive benefits during periods of unemployment.

Training and entry are also significantly affected by the model of production, since firms are unlikely to see the value in financing training, apprenticeships, skills upgrading and development for workers who are only temporarily engaged (Eikhof and Warhurst, 2013). In film and television, production firms have increased their reliance on low or unpaid internships, and young workers often work for months without guarantee of future employment (Apitzsch, 2010; Holgate and Mckay, 2007; Perlin, 2012). Internships have become accepted as a primary way of attaining career entry in the field.

The project-based model of production also has an impact on the way individuals are recruited for work in the creative industries. Uncompromising budgets combined with short turnaround times leave "no room for error in recruitment", and those hiring, therefore, most often turn to "tried and tested" associates or those coming with recommendations from trusted networks (Eikhof and Warhurst, 2013, p.498). Hiring in film and television follows this pattern and is achieved primarily 
through informal networks of connected professionals who compete for, and recommend each other for jobs (Apitzsch, 2010; Holgate and McKay, 2011; WIFT, 2012; Wing-Fai, Gill and Randle, 2015; Wreyford, 2015). Connection to the 'right' networks in film and television has been shown to be one of the most important factors of success and has a significant impact on access to elite jobs (Antcliff, Saundry \& Stuart, 2007; Christopherson, 2008; Christopherson, 2009; Grugalis and Stoyanova, 2012; Lee, 2011; Skilton, 2008). In addition to this, homophily, or the tendency to prefer affiliation with those who most resemble ourselves, has found to be a pervasive feature of human social networking, including in hiring (McPherson, Smith-Lovin \& Cook, 2001). The tendency toward homophily is thought to be even stronger in situations of high ambiguity and uncertainty, which are both commonplace in the creative industries (Jones, 2002). Affiliation with the "in-group" in the creative industries has been shown to be affected by both ascriptive characteristics as well as shared cultural and social capital (McDonald and Day, 2010). The preference for shared cultural and social capital in the recruitment of workers therefore, acts as a barrier to the acquisition of economic capital since it restricts who gets in and advances. This phenomenon therefore perpetuates advantage (and disadvantage) for some groups and not others.

Working patterns in the creative industries are also affected by the model of production. Working hours are long, and often unsociable or in varied and/or remote 
geographical locations (Eikhof and Warhurst, 2013). One study of Canadian television workers revealed that $60 \%$ of respondents work at least 45 hours per week, 8.4 hours greater than the national average (CMG, 2013a). Approximately $30 \%$ of respondents reported having fewer than 12 hours off between the end of one workday and the start of next, and one-fifth indicated that they work more than 10 hours per day (CMG, 2013a). Half of respondents also reported being made to work on evenings and weekends (CMG, 2013a).

These inherent consequences, according to Eikhof and Warhurst (2013) have effects on social equity in the creative industries, and differentially impact some individuals more than others. For one, the organization of work as well as recruitment and training in a project-based model of employment privileges those with stronger economic means. Those who can afford to work without security, benefits and low or no pay, are better positioned for careers in the creative industries (Eikhof and Warhurst, 2013). Importantly, those with limited economic means are disadvantaged right at career entry, and this significantly impacts the composition of the workforce in the creative sector (Eikhof and Warhurst, 2013). In television and film for example, without access to maternity benefits, female freelancers who choose to have families must carry the full scope of child-rearing risks themselves (WIFT, 2012). The fast pace at which technological changes occur in the film and television industry mean that upon return to work, women often face increased difficulty in catching up (WIFT, 2012). Women are 
thus less likely to be offered promotions due to the lost time, and the catch-up phase that follows their return to work. If they choose to have another child, the loss and catch-up cycle repeats again. These disadvantages accumulate over the career lifecycle and are thought to lead to the underrepresentation of women in higher roles, and discrepancies in income (WIFT, 2012).

According to Eikhof and Warhurst (2013), the production model's impact on hiring practices disadvantages women and visible minorities. Women and visible minorities have been shown to have less access to the elite networks that lead to the best jobs across the creative industries (Christopherson, 2008; 2009; Allen, Quinn, Hollingworth and Rose, 2012; Lee, 2011; Grugalis and Stoyanova, 2012). In film and television, visible minorities in particular, have been shown to be lacking in both the strong and weak ties that lead to top work (Lee, 2011; Grugalis and Stoyanova, 2012). Visible minority students in the creative industries have been found to be more likely to choose internships based not on quality, but on duration and even proximity to the home, opting for shorter internships, as well as those that allow them to meet other commitments such as part-time employment (Allen, Quinn, Hollingworth and Rose, 2012). This perpetuates deficiencies in their networks and narrows opportunities for success. Being on the outside of key networks is significantly disadvantageous throughout the entire career lifecycle since these networks structure access to various opportunities, including promotions. Women and visible minorities are also more likely 
to be unable to participate in the networking culture, or be able to work unsociable working hours or in remote locations due to other non-work related commitments including family or secondary jobs that provide economic stability (Allen, Quinn, Hollingworth and Rose, 2012; Grugalis and Stoyanova, 2012; Lee, 2011; Skilton, 2008). Intensified Model of Production in Web Series

In web series production, the rigidities associated with the creative industries model of production, and the film and television one specifically, are intensified. Figure 19 below demonstrates how the model of production is intensified in web series over film and television.

Table 3

TV/Film Model of Production vs. Web Series Model of Production

\begin{tabular}{|c|c|c|}
\hline $\begin{array}{l}\text { Aspect of Work and } \\
\text { Employment }\end{array}$ & Television and Film & Web Series \\
\hline $\begin{array}{l}\text { Organization of work } \\
\text { \& employment }\end{array}$ & $\begin{array}{l}\text {-insecure } \\
\text {-contract-based; contracts } \\
\text { legally binding } \\
\text {-limited access to social } \\
\text { safety net in Canada (no } \\
\text { unemployment benefits; } \\
\text { some access to parental, } \\
\text { sick and compassionate } \\
\text { leave depending on } \\
\text { eligibility and enrollment) }\end{array}$ & $\begin{array}{l}\text {-extremely informal work arrangements, } \\
\text { especially in self-funded web series; often } \\
\text { times workers do not have formal } \\
\text { contracts; level of risk exceptionally high } \\
\text {-in self-funded web series, arrangements } \\
\text { often haphazard, 'whoever can show up' } \\
\text {-in self-funded web series in which there is } \\
\text { no pay, there is no possibility to utilize } \\
\text { hours worked for employment insurance } \\
\text { purposes; due to low budgets web series } \\
\text { typically require ample "sweat equity" } \\
\text { even in paying situations, and this work is } \\
\text { not compensated, and often unreported } \\
\text {-in self-funded web series especially, } \\
\text { individuals typically take on many and } \\
\text { often disparate roles (ex. } \\
\text { cinematography, writing, directing and }\end{array}$ \\
\hline
\end{tabular}




\begin{tabular}{|c|c|c|}
\hline & & $\begin{array}{l}\text { craft services) } \\
\text {-due to low budgets, pay is often below } \\
\text { television/ film industry standards }\end{array}$ \\
\hline $\begin{array}{l}\text { Training and entry } \\
\text { into the field }\end{array}$ & $\begin{array}{l}\text {-internships common but } \\
\text { ultimately temporary } \\
\text { (typically have formal } \\
\text { commencement and } \\
\text { ending dates) } \\
\text {-internships governed by } \\
\text { provincial legislation } \\
\text {-workers must carry the cost } \\
\text { of training and } \\
\text { development } \\
\text { opportunities }\end{array}$ & $\begin{array}{l}\text {-participation in web series is voluntary and } \\
\text { there is no official end to the duration of } \\
\text { unpaid work; often projects are } \\
\text { economically unsuccessful and payment } \\
\text { is not feasible } \\
\text {-self-funded web series are entrepreneurial } \\
\text { endeavours and there is no legislation } \\
\text { which limits unpaid participation on such } \\
\text { series since such participation is } \\
\text { unclassified and operates in the informal } \\
\text { economy under the shadow of legislation } \\
\text {-decision to participate in another's web } \\
\text { series typically based on desire for } \\
\text { training; contributors pay for their } \\
\text { participation with their time/ skills }\end{array}$ \\
\hline Recruitment Practices & $\begin{array}{l}\text {-hiring based predominantly } \\
\text { on informal mechanisms } \\
\text { including 'tried and } \\
\text { tested' past hires and } \\
\text { recommendations }\end{array}$ & $\begin{array}{l}\text {-because web series are entrepreneurial } \\
\text { endeavours, often individuals work with } \\
\text { their friends and no formal hiring takes } \\
\text { place } \\
\text {-web series community is small; individuals } \\
\text { often work according to a barter/ favour- } \\
\text { economy } \\
\text {-on self-funded web series, free time and } \\
\text { access to capital and other resources } \\
\text { determine who is able to engage in web } \\
\text { series creation } \\
\text {-how much time (i.e. unpaid time) an } \\
\text { individual is able to contribute is also } \\
\text { significant deciding factor in hiring } \\
\text { decisions }\end{array}$ \\
\hline Working Patterns & $\begin{array}{l}\text {-long working hours, often } \\
\text { unsociable } \\
\text {-sometimes work is required } \\
\text { in remote geographical } \\
\text { locations } \\
\text {-sometimes work is required } \\
\text { on evenings and } \\
\text { weekends } \\
\text {-unions often provide }\end{array}$ & $\begin{array}{l}\text {-extremely long working hours, especially } \\
\text { given desire to produce TV-like content } \\
\text { for low or no-budgets } \\
\text {-in self-funded web series, work frequently } \\
\text { takes place on evenings and weekends } \\
\text { since many creators have full time jobs } \\
\text { outside of web series creation } \\
\text {-very small number of projects fall under } \\
\text { purview of existing unions }\end{array}$ \\
\hline
\end{tabular}




\begin{tabular}{|l|l|l|}
\hline $\begin{array}{l}\text { safeguards for their } \\
\text { membership in scripted } \\
\text { production }\end{array}$ & $\begin{array}{l}\text {-work is never done on a web series since } \\
\text { web series are a living entity where } \\
\text { constant and consistent social media } \\
\text { engagement is required for success }\end{array}$ \\
& \\
\hline
\end{tabular}

In what follows below, excerpts from the interviews are utilized to provide context, depth and understanding to the information summarized in Table 3, and to demonstrate how the intensified production model in web series leads to continued inequitable participation of traditionally marginalized groups.

Firstly, because web series are a purely entrepreneurial endeavour most often produced with no outside oversight, or accountability or feedback mechanisms, insecure working conditions are greatly intensified. The project-based production model is exacerbated in web series production since ambitions are high and budgets are low or non-existent. Because for so many creators, web series are viewed as potentially career catalyzing ventures and entry points into the established industry, labour conditions on these series are worse than in traditional film or television. As independent creator RJ Lackie observes,

I think the issue is... where you're a scrappy independent web series, where you are getting it done by the skin of your teeth...you're investing more time, because you're not being paid, so it has to be as good as humanly possible to make it all worth the work. 
This desire to make the series "as good as humanly possible" thus results in exacerbated labour conditions. As successful web series creator Samantha Wan notes, "We're trying to make TV on the web with no funding and saying, 'We can do it.'...we can't. Not for free... it's not sustainable".

In order to make things work, web series creators often take on multiple roles in traditionally disparate departments to overcome the shortcomings of low or nonexistent budgets. Figure 17, below, demonstrates the prevalence of taking on multiple roles in the key categories of writer, director and cinematographer. Shared roles are more prevalent in self-funded web series where funding sources are typically even more limited.

Figure 17

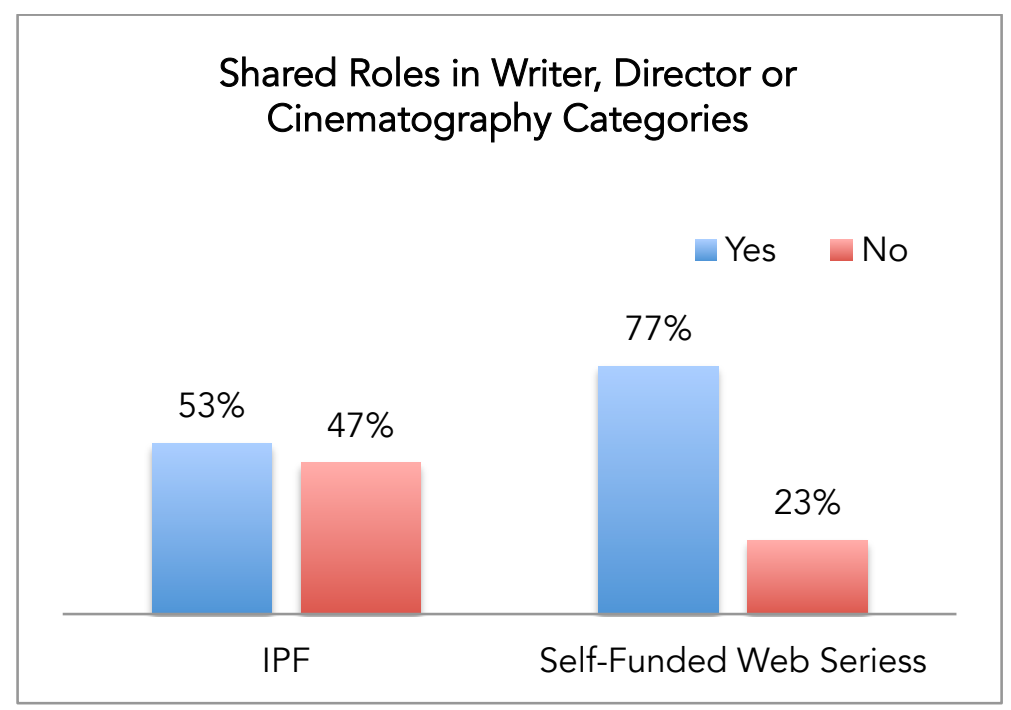


Even with IPF funding, however, achieving the scope and scale of the vision many web creators have is challenging. Jeremy Larter, creator of IPF-funded web series "Just Passing Through" recalled his difficult experience, which required his core creative team to take on multiple simultaneous roles:

There's four of us who wrote the first season, and then Geoff and I and my brother, Jason, produced season one. And I directed the show and edited the show, and then this time around, it means basically the same. Geoff and Jason had to do a lot of producing in season two...So, doing it on the scale that we are doing it on, I don't think we could ever do it again like that. Just because the amount of time and effort and the lack of money makes it hard. The time I had with the show... doing a show on this scale will break you.

Not only does the gruelling nature of the work often lead to exhaustion and burnout, when there is a production budget, creators often forgo their own wages in order to be able to pay hired crew members. Another creator on an IPF-funded web series revealed she did not pay herself any producer or development fees, or any other wages related to the extra work she took on:

I got paid a script fee. [My partner] didn't take a director fee. I think actually, of the above the line people, I think I was the only one who got paid. And I was the only one that got paid because it went so bad for me, because l'd been slaving away for all these years... So they paid me the Writer's Guild scale... But I was the only one I think who got paid that was above the line. I did garbage at the end of the night, and craft services. [My partner] directed and helped to write, of course. He did the website and... I did the website as well...While we were making those web series, we starved.

Another IPF-funded web series creator shared a similar experience: 
We paid our cast and our crew more than we paid ourselves. We primarily paid ourselves for the production. So, when we were on set, we paid ourselves...I got paid for the writing... [My partners] got paid for certain roles that they did. But largely, sitting in the office, typing, uploading, releasing, planning-essentially was volunteer...it was our priority to pay people.

Wages for crew members, when paid, are also limited, and as one creator revealed, are typically "nowhere close" to industry standard. Even when the rates for crew appear fair, once overtime and long hours are factored in, they are not sufficient:

I think the crew rates were all pretty good. The only thing that made them shit is that we didn't pay overtime. So, on certain days, we'd shoot 14-15 hour days, and now, all of a sudden, you have a rate that was okay become kinda shitty. (Independent creator, IPF funded web series)

On self-funded series, crews frequently work on a volunteer or favour-basis. They are often 'paid' in food and transportation, and promises for similar volunteer labour to be offered on their own productions. As one creator noted, "Usually, our crew would be from seven to 12 , depending on the size of the episode...we never paid anybody. And the only money that ever went into it was for lunches and then to buy some props and stuff".

Another creator recalls the amounts he paid his crew on his IPF-funded web series.

The rates were lower than industry standard:

I think for the writing room we paid people $\$ 100$ a day. I think we paid our cast somewhere in the neighbourhood of $\$ 100$ a day...[The hours]...they were insane. 'Cause we were shooting on average 15 episodes in two or three days. (Independent creator)

For many creators, the financial pressure was too much: 
I'm not making another series the same way I did [name of series]. It was so much work, not only on my part, but on everyone involved for no remuneration. It's just not viable...And of course, we covered their transportation, we covered their food. So it didn't cost them anything to do it. (Independent creator)

Participation in web-series, like all entrepreneurial ventures, is self-limited. Individuals engage to the extent that they feel they can. The organization of work in web series is likely most similar to the production of independently produced television pilot episodes or feature films. Creator-driven web series are also mediated by the same factors that are germane to entrepreneurial activity in general. These factors include age, gender, race, social class, social mobility, and family support, and importantlythe intersection of these factors (Beggs, Doolittle \& Garsombke, 1994; Farlie, 2005; Farlie and Meyer, 1996; Fielden and Dawe, 2004; Harvey, 2005; Smith and Air, 2012; Walker, 2009).

Thus, while complete freedom over access to distribution through the removal of formal gatekeepers (the broadcasters), in theory, permits increased opportunities for participation, the same identity-based factors, which act as barriers to entrepreneurial activity in general, also act as barriers to web series creation and participation. While these factors mediate participation in the creative industries generally and are directly affected by the creative industries' model of production (Eikhof and Warhurst, 2013), the high levels of uncertainty and risk involved in web series creation (i.e. self-initiated, self-funded creation, versus work on a firm's funded television or film production where 
financial risk is not carried by the worker, but rather by the firm) increase the relevance of these social factors. All of the independent creators under 30 who were interviewed for the study indicated that they grew up in middle class homes and/ or had direct support from their families while making their series including rental reprieves, living at home with the family, miscellaneous production support (ex. transportation and craft services) and direct financial contributions toward the production of their series. These creators understood that receiving such help alleviated significant financial pressures.

Sarah Shelson, a web series creator and digital production company co-founder in her twenties noted,

I did have support, and I was working like a sort of a 'Joe job'. I am super lucky and privileged that my parents live in Toronto, and I can live with my parents and not have to worry about rent. That definitely helped.

Yusuf Zine, another creator in his early twenties echoed these observations, noting he and his partners were "all really fortunate to be at home", and able to produce their series while not "working or making any substantial income".

Another creator in his early twenties, described his upper middle class family background and the direct contributions his parents provided throughout the filming of his highly-viewed and award-winning web series:

[M]y family is like upper middle class 'cause my dad has one of those jobs like Chandler in Friends where no one really knows what he does, but he works high-up at this bank... And then my mom runs her own business... didn't wanna go to film school initially... But then, they kind of said like, 'If you go, we'll help you out with 
your film projects.'...And it costs a lot of money to buy film stock and just to make a film, it's expensive... They've helped with that and then with [my web series], they helped me fund it... I always made it a point to give everyone transportation everywhere, and a lot of the times my parents would drive actors all around, so they were really helpful with that...And my mom's a great cook... and she helped a lot with that [on set].

Many of the other creators over the age of 30 indicated having full time employment (often in another facet of the entertainment industry) to provide the needed capital to fund their web series endeavours.

Herman Wang, creator of the web series, The Spell Tutor, noted that it is his day job as a computer programmer that makes his web series possible: "I mean, my day job is very good. I happen to be lucky enough to be good at something that pays well. So, I mean, that is actually where most of the funding for this stuff comes from".

For many creators over the age of 30, past experience in film and television has been integral to the production of their web series, not only by providing income, but also by providing key resources. As JP Larocque, creator of the award-winning web series Gay Nerds observed,

So my day job is casting in reality television...And that's been great, because that's offered me the ability to make money and pay rent, but also to do my own creative projects on the side... on my show in particular, we were lucky because the group of us all had careers within the industry, all had complimenting talents. But again, as I mentioned to you, we were working on a Red Scarlett [a high-end cinema camera], we had editing, we had equipment that was available to us because the director of photography owned it, and he had it. Obviously, one has to have a certain amount of money to be able to afford those things. So, I think that it's the reality of the world we live in. 
Independent creator and experienced film producer, Ryan Keller, also

recognized how his work and experience in film was key to the creation of his web series Microwave Porn:

I had the equipment, and it was all professional equipment. I had lighting, I had microphones, I had the cameras, I had the editing systems. I was doing visual effects myself. So, all of that production infrastructure was there. And then we used our connections in the industry to bring in a fairly small, but honed group of people to work with us.

Others were fortunate enough to have spouses with more standard employment that provided much needed stability. One creator noted, "I'm married to a high school teacher...So I can still go to the dentist, I can do that kind of stuff".

Free time, as a mediator of who can participate in web series was also repeatedly brought up by the interviewees. As Ryan Keller explained,

The thing that precludes [participation] in a lot of ways is it takes time. There's no creative geniuses in this industry. It's not painting. You can't sit in your basement. It requires a lot of people and a lot of skills to do it well, and that takes time to develop those skills. What precludes great web content in a lot of cases is that people can't devote enough time to this 'cause they're not making any money.

Several creators explicitly spoke about the difference between vlogging and the creation of scripted web series. Although both are time intensive, scripted web series creation, which as discussed above, adheres to a SoCal logic, and is generally more resource intensive when done well. The privilege of having enough free time to engage was seen as key to being able to fully participate in the genre.

IWCC Vice President Dan Speerin linked the notion of free time to social class: 
Yeah, there's a huge class barrier that's coming with this kind of creation... If you're gonna feed the [YouTube] algorithm, you have to be home. So it's the kids who still live at home with their parents, or people who have the extra money that they don't need to work a job [that are most likely to succeed].

According to many of the creators interviewed, YouTube's algorithm has a built-in bias toward volume of production and consistency. The impact of this algorithm on the chances of success of scripted web series will be discussed further in the next chapter. What is important to note here, however, is the connection between this requirement for volume of production and consistency and free time as a key resource. Those without adequate time are clearly disadvantaged. As creator Jason Leaver noted, Time is incredibly undervalued...So, then the question becomes, well who has time? And the answer to that is probably affluent white men, of which I am that... In terms of [outside] barriers that the industries have, those I think are gone. In terms of barriers of just being able to get it done, those are still there... They might be at the job they hate and they have to do that and so that's that. You're not gonna make a web series if you have to spend $80 \%$ of your waking time doing something else.

As Leaver and other creators observed - the barriers to participation in the web series space are no longer dictated from the outside; there are no editorial gatekeepers that prevent access-the barriers are internal, and a key one is free time. "Time poverty" or the absence of time to pursue opportunities (Lister, 2004) is a concept that is just beginning to emerge in studies of entrepreneurship. Scholars note the demands of unpaid domestic work, for example, on top of expectations to also earn incomes and contribute economically, results in a greater degree of time poverty for women than 
men, and restricts their capacity to pursue entrepreneurial ventures (Pozarny, 2016;

Warnecke, 2013a, 2013b). As sociologist Lister (2004) observes, "Time is a resource that interacts with financial resources" (p.59). Better-off individuals "substitute money for time through purchase of labour-saving goods or the services of others", while those who cannot afford to do so "do the opposite —expending time in order to save money" (p.59). Although time poverty can exist without material poverty, "where the two coexist it represents an additional dimension of poverty and in some cases it may be that material poverty is avoided only at the expense of time poverty" (p. 59-60). Web series creators without sufficient funding expend their time in lieu of finances to complete their projects, often to the point of psychological burnout.

Describing her experience on her IPF-funded web series, one creator lamented about her difficult relationship with the resource of time, noting that the most heartbreaking part of making her series was the realization that despite creating a world that audiences connected with, the "financial implications of time" prevented her from being able to actualize its potential:

It's terrifying [Making a web series]. I hate it. I hate it, I love it. I'm inspired by it. It makes me cry, it makes me laugh and it makes me sad and happy all at once. Because I think we did something that really resonates with [the audience] and they love it. They react to it, they engage with it and I have content ready to go and I have a whole world that I love and that they love and you think that the web makes it all that much easier, it doesn't. Because you know what's valuable? What is valuable is time and you can't get people's time, you can't get people, you can't get the time of an editor or a coder or... You can't get that time...the financial implications of time [are the biggest barrier in web series], let's put it that way. 
Another established creator and founder of a successful digital media company detailed his experiences on his IPF-funded web series, connecting his decision to no longer continue with the project to the value he places on his company's time:

I just think that ...very smart web series have realized that the only way that they can afford to get to a self-sustaining business model is to reduce costs to almost nothing. Now, there are significant costs in time that they contribute, but they rarely are paying externally. I think that there's an interesting position of privilege that comes from that, if you happen to be able to contribute hundreds of hours to the creation of content so that you get to the point where you are self-sustaining, then that's something that not everybody has the opportunity to do... On [series name] the problem was that once we had spent the budget to make something great then it was a question of how much money are we willing to invest in this and we didn't see time as being valueless...And so at a certain point it's like, 'if I put in', and I can calculate because I know my hourly burn rate at the company, and if I say, 'if we put one hour in, we're only going to get a return of $0.1 \%$ '. As the business person, there's no justifiable method by which I can continue that project. And whereas I think that a lot of the projects that have made that realization also realize they can turn it around with just enormous investments of sweat equity...I think there is a version of our path where [series name] could have been what you would think of as a stand off success, if everyone involved had said, 'We love this project so much that we're gonna forego our pay cheques and we're all going to work on it for free until it makes it.'

Another young creator observed a connection between free time, and who was able to participate in the writer's room he created for his web series:

When I did my writer's room, like the fake writers room, practice room, we only had a couple [visible minorities] because of scheduling. 'Cause we had people in the room and then we did the freelance model for people who couldn't be physically in the room. It's just how the scheduling landed and I felt awful about this-all of our non-white writers were in the freelancer zone...And, it was just the whims of numbers, because it was basically during the week or weekend. A vast majority of people can meet on the weekend, but it just so happened that all of our non-white writers couldn't. (Independent creator) 
Due to other commitments, the non-white writers simply could not devote the time required to be "physically" in the room and were relegated to freelancer status.

Tellingly, other creators noted a similar pattern when it came to who could make it out to web series auditions. As one creator observed,

I mean, the leads are all white... That's just the way it worked out, and I mean this, in the end, I would have preferred a more diverse lead cast but we were restricted by the people who auditioned... I would say that the response to our casting call was something like maybe $70 \%$ to $80 \%$ white, and then the rest were like Asian, MiddleEastern, things like that. (Independent creator)

Another creator similarly explained, "We definitively wanted [the show] to be diverse.

Turns out, when we ran auditions, we didn't get a lot of [diverse talent]...And the show that we produced is not the show that we envisioned" (Independent creator). While on-screen diversity in web series was not a metric examined in this study due to limited resources, it is not difficult to see how time and other socio-economic factors act as filters to more inclusive on-screen participation even in the web series space. Free time, therefore, and the implications of who has it (and who does not), act as key barriers to creation on the web. Only those with sufficient free time can fully participate.

Closely linked with time was the factor of age. One creator in her mid-30s reflected on age and its connection with free time:

I literally do not have the time to invest in something that is not making me money, and a lot of people don't, which is understandable. Honestly, I think there's a big 
part of it that has to do with age. When you're younger, you can invest your time and sweat into these projects and it's less important than when you're older and at a different stage in life and need it to actually be a viable source of income. (Independent creator)

Another creator also linked his decision to stop creating for the web with age, noting that he simply could no longer afford the sweat equity and sacrifices involved with maintaining his series, "We're talking about age, that's why I bailed out, because I can't do six clips a day. I would like to have relationships still" (Independent creator).

Younger creator, Sarah Shelson, affirmed the benefit of age when it comes to web:

[T]he nice thing about where we are at, is we are so young that we can take those risks and be like, 'This is fine. We can live with whatever, we can eat ramen for a year and then make this...'

Other younger creators, however, noted that while youth and the ability to take risks

have their advantages, experience also matters:

I think, sometimes, in some senses, if you have some experience in the space, things will go easier for you if you're young because you're the youth...But on the other hand, because so many of these projects come by because of connections, young people typically, don't have the kinds of connections, and a lot of people who can make series happen at the budget levels we need are still in that very middle-aged, [established] system. (RJ Lackie, Independent creator).

Several younger creators even expressed feeling left out of the emerging Toronto-

based web series community on account of their age. One creator noted:

We were very young at the time. And I love the people who are part of the Toronto web series community, they're great people and they really have given us a lot of great advice, but we have never really felt a part of that community, because everyone else in there is a little bit older...It comes across as sort of an elitist, exclusive club. Again, there are individuals in there who are awesome and who have 
been great, but we've never really felt a part of that community because...there are people who are more established, who have the big name web series, they're older...they have funding... and they're on the boards of these sort of committees. (Independent creator)

Despite the challenges, many of the young creators interviewed felt that, all things considered, in the risk-averse climate of traditional television production in Canada, producing a web series is almost an "expectation" to break into the industry. Breaking into the established system could therefore become even more exclusive given the intensified model of production in web series, and its ensuing impacts on access.

Based on the discussion above, the reason for the continued underrepresentation of women and visible minorities across the key creative categories of writer and director in funded and self-funded web series becomes more apparent. Access to web series funding is mediated by a culmination of the various socioeconomic and cultural factors explored above.

The fact that the situation is worse in web series receiving funding is not surprising either. Scott Albert an established writer in the television industry, and a serial web creator in his 40s, explained the issue well:

I think it's a little bit like the old IO test where it's come to light that it's kind of socially and economically biased. I think, 'who are the people who are sitting down and filling those forms?' And also the funding agencies rely a lot on track records, right? And so who are the people who have track records who meet the funding requirements. And more and more, the funding requirements especially for the IPF require some form of distribution partner or some form of production partner. And 
so you have to look at the companies who are starting to smell this money and are coming in...And now it's the same people who are making TV who are starting to get the money to co-finance these web series...it's a vanity to tell stories on a certain level. It's an entitlement, right? There's a sense of entitlement... Because even when you get the funding... The funding money-it's not enough to really make a living doing it....And so, who are the people who are able to invest the nonfinancial resources while not making enough to cover the mortgage year after year who are able to do this? I think there's a lot of social and economic funnels that keep out people who aren't middle class, firmly established with a big support network... (Scott Albert, Independent creator)

This observation that individuals with the right kind of cultural, social and economic capital are more likely and able to access funding helps explain why certain people fare better than others in competition-based funding, especially since the evaluators (metaphorically) "speak the language" of those most likely to succeed. This kind of cultural, social and economic understanding privileges those with what it considers to be 'recognizable' achievements including awards, critical acclaim, credits, existing business relationships and already established deals, and the like. Anyone who cannot meet these expectations is left out.

It is clear how the intensified production model of So-Cal style web series creation presents barriers to the participation of certain groups of creators and not others. While in theory, "anyone can make a web series", not everyone has the capacity to do so. Because scripted web series follow a So-Cal logic, they are resource intensive ventures. The same barriers that act as impediments to entrepreneurship more broadly (age, gender, race, social class, social mobility, and family support) are 
especially germane in the scripted web series space given its high-risk nature. Creators with more than one source of disadvantage experience even greater challenges with respect to being able to participate in scripted web series creation. The high importance of socioeconomic filters in this space, due to the intensified model of production, therefore, mitigates some of the effects of the freedom provided by the nearly unfettered access to online distribution. All web series creation also implies a certain level of web literacy that not all possess. The notion that social media platforms such as YouTube are beacons of participatory culture is therefore, far from universally true, particularly when it comes to establishing a career in scripted entertainment.

Although, as revealed in Chapter 4, the rejection of the status quo with respect to the representation of women, people of colour and other marginalized subjectivities both on and behind the screen spurred the entrepreneuring of many of the creators interviewed, a deeper structural analysis of emergent inclusion patterns demonstrates the persistence and "solidity of the structures [web creators] seek to dislodge" (Rindova et al., p.479). Thus, although some individuals from the traditionally marginalized groups choose to challenge the status quo through the creation of web series, the systemic inequality inherent in the intensified web series model of production acts as an inhibitor to the participation of many others from these groups, and indeed has an impact on the participation of those who engage in the entreprenuring as well. 
By incorporating first, an examination of the subjective goals behind their entrepreneuring, followed by a structural level analysis of the role of social identity characteristics, including gender, race and class, in web series creation, the process of structuration, a central focus of this dissertation, is brought to the surface. From the marriage of CPE with Rindova et al.'s (2009) particular conceptualization of entrepreneuring as an active process fundamentally aimed at change, it is clear how it is possible to "address goal-oriented, reflexive human action, without giving up on understanding the 'sutures' of power that mutually constitute social action" (Mosco, 2009, p.186). The expanded CPE approach therefore makes clear how web creators both utilize their agency, but are at the same time, affected by the structures that exist. The contextualization of subjective goals through the explanatory power of CPE will permit, in the final chapter, the emergence of meaningful policy and program recommendations that address both the ideals and realities that exist.

While this chapter examined internal barriers to participation, the next chapters explore external barriers to success for Canadian scripted series that come from both within, and outside of, the Canadian industry. 


\section{Chapter 6 - Barriers and Challenges from Outside of the Canadian System}

The previous chapters focussed on the motivations of those who decide to engage in entreprenenuring on the web, and also the barriers to participation related to their identity characteristics. Web creators' motivations relate directly to the realities of the world they occupy, and through their work they set out to break free from and to break up a number of rigidities and negative externalities associated with the traditional Canadian screen industry. While it is true that anyone can become a web creator, the creative industries project-based model of production (Eikhof and Warhurst, 2013) is amplified in the web series space due to its unique features, and the freedom to participate is therefore mitigated by the heightened centrality of socioeconomic filters that are germane to the field of entrepreneurship more broadly.

As previously mentioned, despite their grand motivations —-those engaged in entrepreneuring "may have only a limited understanding of the solidity of the structures they seek to dislodge" (Rindova et al, 2009, p.479). This is particularly true in the web series space, where the structures that creators aim to disrupt in the traditional television space not only extend over into the world of the web, but are compounded by a litany of newly emerging (or emerged) structures native to this new world. This chapter is organized as follows: After a discussion of how Canadian web creators' fared with respect to the typical metrics of success, economics (profit) and audiences, the chapter then proceeds to explore the various structural challenges that cannot be 
attributed to the Canadian system alone including larger cultural and social trends, and extra-national forces and institutions.

\section{Achieving Key Success Metrics: Audiences and Economics}

Making money on the web directly from their web series projects is an extremely challenging prospect for web series producers regardless of their status and broader screen industry experience, or their projects' originality, visual quality (some series were filmed by experienced production crews using cinema-quality equipment), critical acclaim (press attention; positive reviews; awards success), or audience size (some with total views in the multimillion range). With respect to economics alone, many of the creators interviewed for this project largely deemed their efforts to be financial failures. A few of the creators interviewed had secured or been offered licensing or commissioning fees from domestic and/ or foreign traditional and digital broadcasters, for as little as one thousand American dollars per season for non-exclusive rights, to over four hundred thousand American dollars per season for exclusive rights to the US market, for example. For the most part, however, the sums received were not enough to adequately cover the production related expenses required to achieve the producers' overall visions, and (unpaid) sweat equity, and low wages, were central to filling in budget gaps. Many creators also admitted to going into significant debt in order to complete their projects. 
For those creators without external funding, revenues generated from their various monetization efforts were generally not enough to cover the costs of production, particularly if all production team members were to be paid full and fair wages, as well as overtime. This was the case regardless of whether there were audiences in the millions or in the few thousands (or less). Producers had to be extremely creative in carving out international rights for sale, and the amount of work required to secure such sales was considerable. As one producer noted, "We more or less broke even on selling the shows to short film broadcasters. We sold it to...Canada. We sold to Russia, we sold to the US, we sold to just a bunch of different broadcasters" (Independent creator). Even in this case, the producer admits that without a substantial amount of unpaid sweat equity, the production could not have been completed. While the production then may have technically broken even when taking into consideration only how much was officially spent, as the producer admitted, a true break even would have required the proper valuation of each team member's contributions and time. Sustainability thus remained a dream, rather than reality for these creators. One notable Canadian exception resulted in over a million dollars worth of merchandise sales in the early years of web video; in this case, the series was not in and of itself profitable, however. For the projects with external financiers (including crowd-funded projects), no projects had broken even in the sense that the revenues generated from the projects returned more than the initial investments made by the various financiers 
after all costs were considered, although a notable (very) small few had made significant progress in this area and were on their way to achieving this important milestone. One creator noted that between his many varying efforts including speaking engagements, taking his producer fees from grants and other funds, and revenues from live events built around his web series, "there's a way to survive... There's just not a way to thrive" (Digital production company startup founder). Branded content, or scripted web series financed in part or in whole by brands are another potential source of financing, but this method is not widespread by any means, and the value of such a venture is difficult to prove for brands (Shields, 2016). Not all series are amenable to being financed in this way as well due to subject matter that might not be desirable to brands seeking a particular association or that may wish to avoid certain "controversial" subjects.

Finding and building audiences was also challenging for many creators, although some did achieve substantial audiences resulting in greater than twenty million total views. Despite having prestigious domestic and international awards, known actors, press, critical acclaim and in many cases, high quality visual elements, no single episode of a web series achieved the viewership of a typical video that has "gone viral". The popular "David after Dentist" YouTube video (Booba1234, 2009) for example, currently has in excess of 134 million views. The web series examined for this project also have not been able to attain, with the same consistency, audiences in 
excess of one million views per episode, as achieved by the most popular makeup and beauty, do-it-yourself, or videogame vloggers (ex. Michelle Phan; Bethany Mota; PewDiePie), or online sketch comedians (ex. Superwoman; Jus Reign; Jenna Marbles). This is the case not just for Canadian web series, but for web series that are selfdistributed in general. The most watched independently distributed Canadian web series have view counts that are competitive with web series from the United States.

Notably, in the 175 web series examined, there is also almost always a significant drop in viewers from the first episode to the last.

In the section that follows, the extra-Canadian challenges that prevent web creators from achieving the kinds of outcomes they seek in the areas of audiences and economics will be explored in depth.

\section{The Challenges}

\section{i. Audience expectations}

Web series creators consistently noted throughout the interviews that one of the greatest challenges they face in their efforts to monetize their series is the unique nature of online audience expectations. As one serial web series creator observed, "I would say the number one barrier is the mentality, the paradigm of our culture to consume entertainment for free, and the expectation that it will be free". He described 
his experiences attempting to monetize his web series by providing 'perks' to those who were willing to pay:

So, four million people watched [our show], 4,000 of them went to JTS [a paid platform] to pay five bucks a month to watch it. Even though we gave them a superior viewing experience-JTS is ad-free, and you get the nice 1080p [viewing quality], and we had bonus content that was exclusive to JTS. So, you'd think, if paying for content was just something you do, that's where you would wanna go first. But, what? $0.1 \%$ of our viewers did that, so...4,000 people from JTS and four million on free platforms.

Despite the added benefits he built into the paid experience, audiences were contented to watch the series with advertising, and in a lower visual quality (not in high definition). Independent creator Regan Latimer agreed, noting that web series creators face a major challenge when attempting to convert online audiences into paid viewers. According to Latimer, this is because "viewers are used to everything being up there for free...so when it comes to paying and supporting it, you have to really freaking love something and really be a huge massive fan". It is precisely because of the abundance of free content that the value proposition for supporting online content (monetarily) must be incredibly high. Many web series producers thus pushed for the creation of fandoms and strove to speak "in the language" of their online communities by communicating through gifs, memes and other non-traditional methods that were native to those viewers.

Jay Bennett, Senior Vice President, Creative and Innovation at Smokebomb agreed that one of the greatest challenges to web series monetization efforts is the 
audience's expectation for online content to be free. Like creator Regan Latimer, he believes that the key to monetizing online viewers is to establish fan loyalty and passion. For his firm, establishing passion and loyalty is most effectively achieved by identifying an audience niche:

Because, again, the rule of the internet is, 'I'll watch it. Okay, that was great. Oh, you're out of stuff? Okay, I'll go to the next button'. You need lots of stuff. ... This mantra, which we have... developed-this is talking about 'millennials' and I'm putting the air quotations around that-the reason I think piracy exists in feature films is because you're asking a group of people to just go and pay for something, out of the gate. And I think there's very little interest in just putting your money down when you have no loyalty to it. I think the new rules of content is niche love for something, and if you love something, it is sort of the music industry model of, 'If you love it, you'll buy the concert ticket, you'll get the T shirt, you'll go on the road trip with them, you'll watch the music videos'....So once you put a paywall up, everyone's gotten so used to the system... 'Oh you want me to pay for it? Yeah, I'll just go and wait 'til someone uploads it'... There's no loyalty. But if there's loyalty, people will say, 'No, no, no. I'm not gonna steal this. I'm gonna go because this means something. I know if I pay, I can get more of it'. (Jay Bennett, Independent production company executive)

Bennett and his team have been able to achieve substantive views in the multimillions for their web series "Carmilla" through this niche strategy. Other creators, however, stressed the abundance of free online content makes it very easy for viewers to substitute their viewing, amplifying the challenge facing those who attempt to monetize their content:

[The internet] creates a very easy substitution. Because if you have a cat video you wanna charge money for, 'well, that's fine but I'm gonna watch [something else], and it's almost as good as yours'. And so there's the substitution effect where there's lots of other stuff and since I don't wanna pay, I can just keep finding things 
that interest me because my starting point is no money. (Brad Danks, Independent broadcasting executive)

Creator Herman Wang noted that not only are online audiences willing (and likely) to substitute paid content with free content, they are also willing to accept "lower quality" content because it is free:

I feel like there's kind of a lowering of the bar too. The audience, they want stuff very cheap or free. And then, I find that, as far as taste goes-they're willing to accept stuff that is very low quality. Low quality stuff does very well, which is frightening because then it's kind of a discouragement for people to try for high quality. (Independent creator, Herman Wang)

Here Herman uses "lower quality" as a catchall term that encompasses content that does not conform to traditional SoCal values. As Herman observes, this acceptance (and success) of content that does not strive for traditional technical and craft excellence, as is the case with most vlogs, cat videos, gaming, makeup and beauty tutorials and the like, disincentivizes the production of independent online content that is more painstakingly crafted utilizing the various time-honoured SoCal components (acting; writing; directing; cinematography etc.). The relationship between the SoCal logic adopted by most scripted web series, and their dissemination on NoCal platforms like YouTube will be discussed later in the chapter. For now, it is important to note that not only are audiences willing to accept substitutions, they are willing to accept substitutions that do not measure up to traditional notions of quality. 
Creator of "Out With Dad", Jason Leaver, decided to try the crowd-funding platform, Patreon, in an attempt to make his popular series sustainable. Patreon's funding philosophy is built around attempting to provide consistent revenue streams for creators from patrons who pledge to support them indefinitely (the platform is built for ongoing, automatic withdrawals based on accepted terms) (Patreon, 2017a). Leaver noted he was apprehensive in switching to a model such as Patreon because, ...it is a huge statement, but it also means changing the mindset of society. Because I'm asking the audience, 'Here's a show I'm giving to you for free, could you please chip in as much as you can?'. It's not just me saying thank you. It's everyone in the world who watches the show who can't do it, they're thanking you [the patrons] as well. So, we are talking about a patronage system which is a hundred years old, thousands of years old, but about a hundred years ago it stopped happening. And then I say to the audience, 'Invest in us, and we'll give you more, Out with Dad', and they say, 'What the hell? Why aren't you [consistently releasing content] Thursdays and Tuesdays like Carmilla [the branded web series by established digital production company, Smokebomb]?' Because we can't afford that.

Leaver's monthly income from Patreon was $\$ 2681$ at the time of writing, $\$ 2319$ below his goal of $\$ 5000$ which would permit him to "pay our cast and crew closer to industry standard wages" (Patreon, 2017b). Despite the failure of most web series to generate the kind of sustainable revenue required to fully fund content, one web series producer noted that creators are perpetuating audience expectations for free content out of desperation to disseminate their work:

We expect everything online to be free, and then the thing that is following that is that we are catering to that now because we're so desperate to get our work out. We're desperate to get work. So I agree with the people who are being like, 'Web 
series could kill this industry', because honestly, I'm in a situation where I'm like, 'You want me to make more content, you have to pay me'. But there's gonna be another guy who's like, 'I'll do it for free', because they just want their stuff out...So, you can get a lot of free content. Not only do we expect it, we're perpetuating it. (Samantha Wan, Independent web series creator)

Audience expectations that online entertainment should be free significantly inhibit creators' monetization efforts. However, as Samantha Wan points out, these expectations are then perpetuated and indeed legitimated in a vicious cycle, when creators, desperate for screen industry opportunities, disseminate their work free of charge in the online space hoping to overcome the odds and also to generate admiration, attention and other rewards.

\section{ii. Form}

Restricted by audiences' expectations for free-to-access content, and determined to reach as many potential viewers as possible, most of the web series creators interviewed turned to YouTube, the world's preeminent free-to-access online video service. According to the company, it has over a billion users, is accessible in 76 different languages, and "reaches more 18-34 and 18-49 year-olds than any cable network in the U.S" (YouTube, 2017a). The scripted web series format, however, which generally prioritizes the time-honoured SoCal crafts of writing, directing, cinematography and acting, faces immense monetization challenges on the platform, which will now be explored. 
As the YouTube guide to generating revenue states, creators should strive to make "consistent videos" to engage audiences (YouTube, 2017b). Many of the web series creators interviewed realized through their efforts that the complex and resource intensive nature of scripted web series production places them at a critical disadvantage with respect to their capacity to consistently and frequently release content.

As one creator, established in the traditional screen industry, observed,

YouTube isn't a particularly great place for scripted. ...YouTube is a volume place. The people who become YouTube stars put out several videos a week on a schedule for years to build that audience. And in scripted, you don't produce enough content to do that. (Jill Golick, Independent creator).

The high cost of producing scripted, narrative programming thus prohibits the production of a large volume of content that can be released both constantly and consistently. Scripted web series creators thus realized that 'organic' YouTube forms such as vlogs are more aligned with monetization on the platform.

As one creator argued:

If you want to make money off YouTube, don't make full scripted series with multiple locations and many actors-just talk to the camera and videotape yourself playing video games and making funny comments. That's the way to make money... Or give make-up tips...there's really no upfront cost, no capital investment to making vlogging stuff. You can have a constant stream of new content. (Independent creator) 
Many creators understood that volume of production is inextricably linked to

monetization capacity on the platform because the greater the number of videos, the greater the number of vehicles for potential ads to be served. As one noted:

I mean, you've got the fact that if you're getting one ad at the top of every episode, and you've got a whole bunch of short videos, obviously the ad share is gonna go up...But I can't take my episodes and cut them any shorter without damaging them. So, that just doesn't work. (Jonathan Robbins, Independent creator)

Another explained:

If you're Jenna Marbles and you're just a genius comedian, then you can set up your little webcam, bring your puppy on, say stuff that's got really good writing that you created yourself, that you shot within an hour and put it online, and you can do an episode a week. Now you have more content because YouTube pays per view. It doesn't pay per how much people like it. If you are releasing eight episodes for the year, 'cause that's how much it takes to do [our show]...well, at most, you're gonna earn over the course the year what Jenna Marbles earned over the course of a month, and she produced it for actually $\$ 0$ of her pay versus you had to actually spend 300 grand upfront. (Independent creator)

While the creator above has likely understated the amount of work that goes into the production of a vlog, there is no question that direct-to-camera, single person YouTube series generally require fewer resources than scripted, narrative content.

The resource intensive scripted web series format also presents challenges to building audiences on the platform. Even before her web series was released on YouTube, one independent creator already knew her five-episode series would face challenges in growing its audience:

So web series need about 30 episodes... Because on the internet, we just eat things so quickly and if you're not releasing a bunch of content, you lose engagement with 
your audience. So the fact that we only have five episodes doesn't make for a great YouTube series. Because, what? We engage people for five days, and then it's over. We get lost, right?.. [We're] 100\%, at a huge disadvantage [compared to vloggers]. (Independent creator)

According to another creator, "you have to be making a video a week...or more to build your fan base and to keep it going" (Jason Leaver, Independent creator). An additional issue that likely acts as an impediment to audience growth is the ongoing nature of most narratives in scripted web series. These programs are typically not made in a procedural style and require considerable investment from the viewer in order to get the full impact of the story. This means that viewers cannot jump into a later episode without watching prior ones, unlike most vlogs, which do not require a specific entry point, and commitment to every episode. As one established web creator commented:

There's a phenomenon that I see in almost every scripted web series...you will have monotonically decreasing views throughout a season. So, we have our season opener... and then it goes down. Then in season two down, and then season three. But our season openers are also monotonically decreasing if you chart that. And that is because the best kind of content for YouTube has an entry point at any episode. You don't wanna have episode seven, and it's like, 'Oh, do I have to know the first six? Too much time investment, I don't know if I'm interested in this.' It's that kind of attitude. So of course, more topical content, vlogs... it doesn't matter where you start. (Independent creator)

This creator's observation that web series viewership declines over the course of the season was, as mentioned in the previous section, confirmed by this study's findings and was true for each of the 175 web series examined. Despite the general challenges, 
however, some web series have been able to successfully grow considerable audiences on YouTube. Web series "Teenagers" by M.H. Murray, has managed to build a substantial audience, with nearly 6.5 million total views for the series' YouTube channel (Teenagers, 2017). Murray credits timing, and also the incorporation of established and emerging talent with existing online followings into the series, for his success:

I really do believe if Teenagers was made with non [union] actors or just a bunch of actors that nobody really knew, I don't think it would have been as successful. But yeah, two of them are from Degrassi, one of them, Chloe Rose has a rather large following. She definitely was a big part of people watching the show, but a lot of the other actors are really big for Canada....up and coming in Toronto. (M.H. Murray, Independent creator)

Murray says he was especially set on securing an actor from the globally popular television series "Degrassi" from the outset, which targets a similar demographic as his own show. Thus, although in general, audience building on YouTube is particularly difficult for scripted web series given that less content provides creators with fewer opportunities to grow audiences over time, exceptions are possible under the right conditions.

Another factor that likely worked to Murray's advantage was the youth-oriented nature of the series. Many of the creators interviewed noted how YouTube has a younger skewing audience, and this presents additional challenges for web series that do not speak to these younger audiences. One established creator observed:

There's a certain expectation of the type of YouTube show and the YouTube audience is very young...I don't think the scripted narrative web series is suitable in 
2015 for the YouTube platform... an eight year old isn't going to watch scripted web series. An eight year old's gonna watch someone playing a game with a funny voice or whatever...or they'll watch fail videos... On YouTube, 'cause the audience is so young, you've gotta be Disney, you've gotta have appeal to everybody. You've gotta have that weird balance between entertaining the adults that are taking their kids to the movie theatre and primarily entertaining the kids, right? There are whole genres of videos that have evolved and been sort of naturally selected by the YouTube numbers. (Independent creator)

Another creator commented,

So our niche is getting buried alive and it's going towards youth culture. It's going towards that MTV culture because they are the ones who A, have the time to make videos, $B$, have the time to consume videos, and $C$, that's where marketers will spend money on, and you can live off that. (Independent creator)

YouTube has been shown to be more popular amongst 13-24 year olds than paid television services (Defy Media, 2015). The business case for catering content to this demographic is therefore clear, and it is not surprising then that many creators reported perceiving a youth oriented focus amongst the top YouTube channels.

Although according to the creators interviewed, YouTube audiences tend to respond to more episodes, which for scripted web series often means more shorter episodes due to economic limitations, high destination buyers appear to be attracted to the opposite form. One established creator whose web series garnered many prestigious awards, including an International Digital Emmy Award commented, [W]e live in that world, where it seems to me that the formats that are valued are longer formats-which is the television half-hour or the television one-hour...I'm starting to think that this idea of sitting down and investing emotionally in characters and a story 10 minutes at a time is not how humans function somehow, 
like I'm starting to think that you need more. You think that all the

millennials...have...short attention spans... but the fact is, is that they're all watching television series, half-hour and one-hour television series online and traditional, however they're getting it. The fact is, is that this is a format they're watching... And so, in my mind, I keep thinking, delivery platform aside, I feel like for drama, for scripted, the half-hour format or one-hour format seems to be where people are at right now. So I just feel like the short-form thing is kind of like almost a non-starter now...We've had all sorts of attempts to take this content out and sell it to all over the world, and I just don't think anybody wants short form. I just don't think short form works... Netflix isn't putting short-form content up... Those guys know where it's at, right? They're doing the half-hour and 60-minute shows. That's what they do. That's what HBO does. That's what they're all doing because that's where their audiences are. (Independent creator)

Many creators reported challenges when attempting to sell their short-form web series.

According to these creators, high destination buyers such as Netflix are simply not interested in the format. Several creators re-edited their series into fewer, but longer episodes or into-feature length films to attract potential purchasers. As several explained, this method does not work for all web series since some have been designed with shorter story arcs in mind.

iii. Digital Dimes, Analog Dollars and Digital Efficiency

According to many of the creators interviewed, both advertiser perceptions about the value of online audiences, as well as a presumption of the efficiency of web-based content have a negative impact on the digital production economy. 
One creator who produces commercials for both traditional television and the

web says the difference in the valuation of the television audience and the online

audience is striking:

McDonald's will pay hundreds of thousands, millions of dollars to reach [an] audience through a television show, yet they could take those same dollars and reach people online at a fraction of the cost and get 50 times the audience, yet that isn't valued monetarily the same...[A] couple of years ago if you got a million views on YouTube and you had a revenue share with YouTube, then that would pocket you [around] $\$ 2000 \ldots$...that's for a million eyeballs. If you get a million eyeballs on television, you have one of the most successful shows. People are paying hundreds of thousands of dollars to advertise on it, and yet online, you can literally reach hundreds of millions of people. Now, I know it's more scattered and whatever [the audience], but the fact is, is that the trackability is insane. I think... we need the salespeople to be out there saying, 'Look-your million views on television are equal to a million views online. And in fact, it's even better.' (Independent creator)

Many interviewees stressed their belief that advertisers are being short-sighted when it comes to their assessment of the value of online audiences. They were of the view that advertising on the web has the potential to be much more effective and efficient than in the television space. One broadcasting executive explained:

In terms of CPM [cost per thousand] what you get is for sure much lower on digital. But that being said, just the reach itself in building audience is way more important, right? You look at any of those digital publications, like you look at Vice, or you look at BuzzFeed, they're able to go an inch wide, a mile deep with one age group. They're able to very, very, very quickly monetize. So whereas in broadcast, it's about scaling as many groups as you can, the larger the range and very, very shallow. At what point is it gonna switch? I think because advertising is getting smarter, they're gonna prefer people that are closer to what they identify and need that they actually have. I would spend way more to know that if I'm gonna reach the audience that's going to buy my bed frames or whatever... (Broadcasting executive) 
Another digital production company executive agreed, noting another potential efficiency made possible though web-based advertising: "The industry hasn't figured out that digital advertising is actually insanely valuable, because you can get people to convert on the same device" (Evan Jones, Independent digital media production company executive). According to the executive, this is because the path from ad view to purchase is vastly shortened, given that viewers can more easily purchase products online.

While television audiences are still valued more than digital ones, the production company executives interviewed acknowledged that the value of advertising on traditional television is declining - "every trend line that you see, is that television and traditional advertising is becoming less and less valuable" (Independent digital production company executive). This is a problem, according to many of the interviewees, because the perception of the value of digital content is not increasing at a fast enough pace to make up for the shortfalls from the declines in television advertising.

One independent production company executive who also works in producing commercials commented:

I know TV is becoming less and less of a thing and people are cutting cords, but we're kinda cutting off our own arms. We're lowering television when we should be raising digital and saying, 'You guys, if you wanna sustain your giant advertising firm, you gotta tell people.' It's like a sales job. Whether it's real or not, I don't know, but the fact is, is that if you wanna have budgets where people actually get 
paid a living wage, then you've gotta tell people, 'This is what it's gonna cost.' Anyway. So to me, that's like a primary of importance. That's huge. That has to shift...And until we start saying that, until we start saying collectively that it's gonna cost money to make something for online. I mean I get it all the time in the advertising world. If you're doing a 30-second TV spot, the budget is this. And then they'll go, 'Okay, but we essentially wanted to do the exact same thing for online and the budget is this'. I've gotta bring the same crew, I've gotta get the same cameras, I've gotta do all the same stuff essentially...Well, okay we'll do it without maybe quite as many people, but it's... I don't know. It's just that there's this weird disconnect. (Independent production company executive)

The perception that online audiences are not as valuable, therefore, is contributing to the decreased budgets allotted to digital content. Another production company executive worried, "I think the danger within this is when you give more for less, why would anyone go back?" (Jay Bennett, Independent production company executive).

Compounding the issues, is the fact that while advertising is still largely local, web series audiences, especially on YouTube, span the globe. According to many creators, this is a key contributing factor to the devaluation of large online audience size. Local advertisers are most often interested in local markets and local viewers, while viewers of Canadian web series often come from many different jurisdictions.

In addition to the problems around the perceived value of online audiences, many creators noted that there is a perception among program buyers that web-based content is somehow innately more efficient and cost-effective than content made for television. One production company executive expressed his frustration about the situation this way: 
[l]t's funny. I get the sense that there's this change going on where everybody always says, 'Digital series. Oh, you can make them so much cheaper.'... There's no difference. The only thing is that we're eliminating is we're trimming all the fat. It's like there are no more fat cats sitting in their trailers. That's the change, really. It's not like there's a new science here. Rent all the equipment, you hire all the people. You pay for all the bullshit you have to pay for and you film the shit. (Independent production company executive)

Another noted, "Until we smarten up and start saying, 'Okay, we're gonna charge equally on other side,' then the fact is... digital will always be stuck in this quagmire of no money" (Independent production company executive).

\section{iv. Online Saturation and Discoverability}

The saturation of online self-distribution platforms also has definitive impacts on the capacity for creators to reach and build audiences. IWCC Vice President Dan Speerin, has observed a marked change in the state of play since his career as a YouTube creator began in the early years of the platform. He says that YouTube has been

"flooded" and that the "signal-to-noise" ratio works against independent creators:

Because there's so much noise, you have to be making content all the time. So I'll give you an example. Somebody in The Young Turks (TYT) Network who's trying to keep up, is waking up at nine in the morning and going to bed at 10 at night, and trying to churn out about seven clips a day, because that's the only way you're gonna make enough money. So that's the downside. If you have a day job, it's really hard now unless you make brilliant content, or you're really gorgeous and somehow have a hook, or something, it's really hard to make one video a week, and be a superstar. Whereas, when I started on YouTube, you could do almost anything you wanted and there weren't enough people there, so you could get away with it. I was one of the first YouTube partners, and was going on television talking about 
what YouTube was. We were making like a video, a couple videos a month, maybe. Now, it's like Monday, Wednesday, Friday, or...

The requirement to keep above the "noise" is difficult for all web creators, but especially so for those who produce scripted, narrative web series because of the vast resources required to do so. Film producer and web creator Ryan Keller noted that in the early years of web-based self-distribution there was a sense of immense optimism, of "the future being wide open", of "new audiences" and a "new world". Now, according to Keller, and indeed many of the creators interviewed,

[T]here's not a lot of success stories to come in and say, 'Oh, these guys did a web series and they got big.' Early on, there were the guys who would do these digital shorts, these digital series, re-purposing video game assets into a-live action short. 'Oh, my God, they've got a deal'... and it drove a naïve ambition, and it just became completely over-saturated. And now, we're like mould growing on an orange. (Independent creator, Ryan Keller)

Some of the interviewees who participated in the early years of web video attributed their success to an early mover advantage, a lack of competition:

[The terrain] is vastly different than 2004, because in 2004 you had the inherent novelty of having a web series... There was a real lack of competition, and especially the kind of content that [our show] was. It was real... It wasn't sort of the Hollywood version... So that really bolstered our numbers, but today there's a lot of noise out there. There's a lot of competition. And you will always be competing with dogs on skateboards and slinkies on treadmills, which will always be out there, which you can't compete with when you're dealing with tens of thousands of dollars per episode of production. (Independent creator)

In addition to being faced with a saturated online market in which content can easily be substituted, the sheer volume of content presents fundamental challenges to getting 
content seen by audiences. The getting seen component (i.e. the 'discoverability' of their content) was a major challenge for web creators. Creator Yusuf Zine observed, "The hardest thing about making a web series is not the production stuff, it's getting people to watch it. There's so many great web series on YouTube that...that are amazing, that have 200, 300 views, and nobody watches".

Reflecting on his Shakespearean inspired web series "The Soliloquies of Santiago", rising web series writer and creator RJ Lackie commented, "we know who our demo is, we just haven't found a way to communicate with them...I don't think the market rejected us; I just don't think they know we exist yet". Others described the process of self-distribution of a web series as similar to self-publishing a book. Many creators said that shows produced by major Canadian broadcasters would face the same troubling fate if suddenly dropped on YouTube:

Basically it's kind of like this; if you took a show that's on a major broadcaster... and just put it on YouTube, how would people find it? And do people like it enough that they're willing to then show other people that will then like it enough that they're gonna watch it and then also show other people? (Jeremy Larter, Independent creator)

Established television writer and web creator Scott Albert believes that independently released scripted series are challenged in the area of discoverability because they still do not have a well-known and straightforward path for mainstream audiences to find them: 
I think the problem with making money off of web series is that it's still missing that connection with an audience who isn't driven to seek them out. So, many people that I talk to that are just in whatever walk of life say, 'How do I find a web series?' And having been in web series since 2006, I still have to say, 'I don't know.' How does the average person come across a web series? I don't know. How do you get your web series seen by an average person who is happy to spend money on going out to movie, who's happy to spend money on buying a DVD, who's happy to pay for cable subscription. How do you get your web series in front of that person? I think until a web series is paying to have posters in the subway, you're not gonna reach the audience who looks at posters in the subway.

Platforms that specialize in the (self) distribution of scripted, narrative web series do exist (ex. weberieschannel.com). These sites, however, do not have broad reach, and their audience base is simply not comparable to that of YouTube. On YouTube, there is no option to browse through only scripted, narrative web series. To provide context to the problem of discoverability on the platform, running a query on "web series" in the YouTube search field yields 33500000 results. A more narrow search, "web series LGBTQ", yields 133 000. The odds of being discovered through search are therefore not high. Emerging platform for video content, Facebook, currently has even more limited search functionality than YouTube. One twenty-something creator described the situation in which web creators find themselves as oxymoronic:

It's like simultaneously the best and the worst thing that's happened because there's so much content which is incredible. Everybody wants to talk about the democratization of media. But then you realize that so much is being lost because of that same abundance. It's such a weird terrain to navigate. (Jamaal Azeez, Cofounder, Independent production company startup) 
Web creators are thus faced with an unusual state of affairs: on the one hand, the democratization of media has allowed them to participate in culture in a way they have never been able to before by enabling the distribution of their products; on the other hand, it has also led to the current state of saturation in which it becomes increasingly difficult, with each new video uploaded, to build audiences, compete, and therefore, generate revenue.

As all interviewees noted, "getting above the noise" requires considerable financial resources for marketing, and the time and skillset required to carry out intensive, digitally savvy campaigns.

v. YouTube

As the main platform through which web series creators distribute and often (attempt to) monetize their work, YouTube, and the complex ecosystem and politics it has engendered for creators, was cited as a challenge by almost all interviewees.

From a monetization perspective, web series creators unanimously reported unstable and low revenues from YouTube's ad revenue sharing program. Currently, YouTube pays creators $55 \%$ of the net revenue generated from ads served on their content on the platform (YouTube, 2017c). If a user skips an ad, the view does not count, and is not monetized. YouTube's terms of service prohibit the public disclosure of CPM rates on YouTube, however, industry analysts as well as creators have provided 
a good picture of average rates (YouTube, 2017c). What is clear is the CPM rate on the platform has decreased year-over-year, and the trend is not expected to reverse (Baron, 2013; Green, 2015). The sheer volume of content, as well as competition in platforms (ex. Facebook; Twitter; Twitch etc.) is thought to have created a buyer's market for advertising (Cunningham, Craig \& Silver, 2016; Green, 2015). According to the interviewees, one can expect to earn approximately two thousand dollars per one million views (before Google takes its share). As the interviewees mentioned, this figure is highly variable and is affected by CPM as well as the status of the YouTube channel (i.e. the number of subscribers; how engaged the channel's users are, etc.) This average figure has been corroborated by other industry observers (ex. Edwards, 2014; Saha, n.d.).

Creators expressed their frustrations with the monetization capacity of the platform:

We've had a very bad experience with it, we enabled [ads] before our [celebrity] episode which is our most viewed episode, in the hopes that would help pay back for the cost of an episode, and yeah we're at 1.3 million views. I think we have made about $\$ 1000$ dollars. It's really weak, and it's to the point where it's not worth it. (Founder, Independent production company startup)

Many creators report receiving "dollars for each video". On top of this, frequent and unceremonious changes to the platform's algorithms create a volatile and unpredictable playing field for creators and their capacity to generate revenue. As "Out with Dad" creator Jason Leaver recalled, "There was a time when we made as 
much as $\$ 800$ in a month, and that was pretty sweet, but then...they changed their algorithms, and now we're making 50 bucks a month".

Reflecting on his long history with the platform, Dan Speerin commented, When I started out on YouTube, it was all about wanting to be on the front page of YouTube. Then it was all about how many views you had. Then it was all about how many subscribers you had. Now, it's all based on time watched. That's what you want, you want people to watch your whole playlist... (Dan Speerin, Vice President, Independent Web Creators of Canada)

A broadcasting executive agreed:

So YouTube has this new model where they push to consistent engagement versus around tent pole program framing. So instead of pushing to one big spike, they want you to constantly have content and maybe occasionally have a larger piece to champion. So they favour that in their algorithm, as well as the more engagement you have around your channel on a daily basis-that favours your surge as well. (Broadcasting executive)

As Van Dijck (2013) notes, it is a myth that YouTube's "content flows unmediated", and is instead, "heavily steered by search engines and ranking algorithms" (p.113). The impact of these systems on web series creators is significant. According to the creators interviewed, content that fits a paradigm of constant engagement is preferred by YouTube's algorithmic ranking systems and is more likely to be recommended and given top billing in search results. The "natural selection" process that occurs on YouTube is highly mediated, and its programming favours certain methods of distribution and engagement over others. Interviewees were deeply concerned by the asymmetrical nature of the power relationship between themselves and YouTube, the 
corporation. Carly McGowan, an executive at the Independent Production Fund,

commented on the challenges presented by this situation:

It's a rocky relationship. You need YouTube; your audience is on YouTube. So you're competing against it... They have all the power and control, and if they decided to change the way they structure things tomorrow they can, and everybody has to just adapt. And that's a challenge for us, 'cause the content isn't a natural fit. But it's also a challenge for the vloggers who are making their primary living on YouTube, because they are risking, basically their livelihood on a corporation that could do whatever they want. (Carly McGowan, Manager, Independent Production Fund)

As Dan Speerin noted, "The money is in keeping people on the site... and this is where I have real problems with YouTube, because you start to tailor what you do-you're chasing the algorithm's tail all the time". Established creators, and those who can adapt their content in the preferred way are at a significant advantage:

It's like we're powerless in a way, against algorithmic thinking and the platforms... I struggle to find the way to express the sort of frustration with not being able to get your content out there because it has sort of been algorithmically sorted as less important than the content these guys have put out because they're always gonna be featured, and if we come out with something new, we're just gonna be so far down. (Jamaal Azeez, Co-founder, Independent production company startup)

For those who choose to build their businesses on the volatile platform, the risk is significant. Others, however, do not even have the capacity to monetize because of YouTube's rules on "advertiser friendly" content. It's guidelines state the following:

Advertiser-friendly content is content that's appropriate for all audiences. It has little to no inappropriate or mature content in the video stream, thumbnail, or metadata (such as in the video title). If the video does contain inappropriate content, the context is usually newsworthy or comedic and the creator's intent is to inform or entertain (not offend or shock). (YouTube, 2017d) 
According to the guidelines, "non advertiser friendly" content includes:

- Sexually suggestive content, including partial nudity and sexual humor

- Violence, including display of serious injury and events related to violent extremism

- Inappropriate language, including harassment, profanity and vulgar language

- Promotion of drugs and regulated substances, including selling, use and abuse of such items

- Controversial or sensitive subjects and events, including subjects related to war, political conflicts, natural disasters and tragedies, even if graphic imagery is not shown (YouTube, 2017d)

Despite YouTube's stated exception regarding allowing the monetization of

"inappropriate or mature content" so long as the "creator's intent is to inform or

entertain" (YouTube, 2017), many get caught in the crosshairs of the policy. Emerging

creator M.H. Murray's popular dramatic web series "Teenagers" often depicts explicit

scenes meant to inform his audiences. Murray recalled a specific instance when an

episode in which two women were discussing the difference between "sex and

fucking" was taken down:

I had to do an appeal to Google. I was like, "Please don't... Please reinstate my video because it's not pornography.".. For me, it was awful because the amount of time we put into it. That episode was 17 minutes long. The amount of time we put into it, when it got taken down, it was such a letdown.

Since then, Murray says he's careful about what he chooses to monetize:

And sometimes I choose not to put ads if I know it's kinda risqué... Google, I've had them message me. Now they actually keep tabs on me, I think, 'cause they message me when I put out episodes, and they're like, 'This could violate, if you choose to promote it.' Like, 'This is too sexual, we can't promote it.' And sometimes it works if you just put it in North America and France, or something. Because Middle Eastern 
countries and stuff like that, they don't tolerate any sex at all, on ads. And I get so frustrated sometimes when... We put so much time into this content, and the fact that there's other videos on YouTube that are really questionable with their content, and they make money...I guess it comes down to why people say you shouldn't make these big dramatic productions, is because the web is so fickle. You never know what could happen to your video. And the more time you put into it, the more is at stake.

Thus, for some creators, the choice is to pre-emptively censor their content by producing only what is sure to be deemed advertiser friendly, or to forgo the chance of monetization completely, but to keep their message and vision intact. Dan Speerin worried that with its prioritization of advertiser interests, YouTube, has in effect, "turned a generation of creatives into a generation of marketers" that only "sometimes... accidentally, luckily fall into creativity". RJ Lackie, creator of the LGBTQ literary web series, "The Soliloquies of Santiago", took a strong stance on YouTube's advertising restrictions, and noted that for him, "In order to have the no shackles Santiago has, we've had to forego [monetization] entirely".

YouTube also came under public scrutiny for its treatment of LGBTQ content in early 2017 when it surfaced that with its "Restricted Mode" filter enabled, many LGBTQ-oriented videos were being removed (and not displayed) in search results (Watson, 2017). According to YouTube,

Restricted Mode is an optional setting that you can use to help screen out potentially mature content that you may prefer not to see or don't want others in your family to see. We use many signals-such as video title, description, metadata, Community Guidelines reviews, and age-restrictions-to identify and filter out potentially mature content. (YouTube, 2017e) 
YouTube creators from around the world began tweeting their experiences, and the hashtag "YouTubePartylsOver" began trending on Twitter (McBride, 2017). In a response, YouTube commented that Restricted Mode is used only by "a very small subset of users who want to have a more limited YouTube experience", and that "some videos that cover subjects like health, politics and sexuality may not appear for users and institutions that choose to use this feature" (YouTube as quoted in Watson, 2017). Once the public uproar reached its height, YouTube issued another statement in which it concluded that the "bottom line is that this feature isn't working the way it should" and that the issue would be "fixed" (YouTube as quoted in Watson, 2017). Whether by intention or by accident, the situation vividly illustrates the substantive and unmatched power YouTube has to structure both distribution and discovery, and to make changes that have material impacts on creators and their work.

Earlier in 2016, YouTube also came under fire for what appeared to be a sudden shift in its monetization policies, which resulted a large number of creators unable to monetize the kind of content they were able to in the past (Robertson, 2016; Romano, 2016; Spangler, 2016). Apparent changes to its algorithm altered how the system categorized "advertiser unfriendly" content (Robertson, 2016; Romano, 2016; Spangler, 2016). In March of 2017, YouTube broadened its demonetization policy again after an investigation by The Times unearthed information that, "Advertisements 
for hundreds of large companies, universities and charities, including Mercedes-Benz, Waitrose and Marie Curie, appear on...YouTube videos created by supporters of terrorist groups such as Islamic State and Combat 18, a violent pro-Nazi faction" (Mostrous, 2017).

The investigative report sparked an unprecedented advertising boycott by more than 250 brands including McDonalds, L'Oreal, Pepsico, Nestle, Starbucks, Walmart, AT\&T, Heinz, Toyota, Audi and many others (Rath, 2017a) and, according to analysts, will cost the company 750 million dollars in revenue (Rath, 2017b). Since the stricter policies have come into effect, some of YouTube's top earning creators, and many others have reported having large numbers of their videos suddenly demonetized and prohibited from earning income (Solon, 2017). In YouTube's heightened attempt to rid the platform of the bad actors, it has affected many apparently policy-abiding creators' livelihoods. This recent state of affairs illustrates the tense relationship between corporate, and creator and creative interests. In a blog post to its creator community, the company noted that although,

[s]ince our founding, free expression has been one of our core values, allowing creators to share their ideas with over a billion fans from around the world...there's a difference between the free expression that lives on YouTube and the content that brands have told us they want to advertise against. (YouTube, 2017f).

How YouTube handles this recent situation will no doubt have major impacts on the shape and form of content that will be produced. 


\section{vi. The Cooling of the 'Wild West'}

The increasing dominance of certain platforms and the general and widespread deepening corporate grip on the structure of, and content on, the internet worried many of the creators interviewed. Established creator and IWCC co-founder, Rob Mills, expressed his frustration over the increasing commodification and commercialization of the internet, and the disappearing dream and promise of the "open web":

Well, the fact that the only reason we're able to do what we can do is because of the open web. And all these other things that are coming down the pipe or any kind of so-called, well-intentioned government efforts to control, or... 'To civilize the internet'. Oh really? Oh, you wanna make it just like the real world? That's not civilized. Those efforts to constrain what's going on, or the effort to turn the net into a mall, whether it's an AOL or a Facebook controlled, or a Google thing, a place where this is all that you're allowed to see is absurd. It's just setting up a different group of gatekeepers. So it's not just the ability to make your show, it's the ability for your show to be seen. It's not just your freedom as a creator, it's the freedom of your audience to find you. So now you see this consolidation, you've had all these players...All these different portals that you used to be able to go to just as an independent, they're gradually winnowing away and you're gonna end up with three key players, be back to the old days of television again with three networks.

Dan Speerin commented on the contradictory set of consequences that, in practice,

emerge when the web is open:

When there is so much choice, there is no choice. You have to have these things, these hubs. You look at Internet traffic, a Venn diagram of Internet traffic, it's going through the same...sources. There's so much power in consolidation right now on the web and what you see that you almost just have to go through those portals...So I mean, that's the real problem with it all, is that there's five places to go. (Dan Speerin, IWCC Vice President) 
Thus, according to Speerin, and many others-infinite choice naturally lends itself to oligopoly since users looking for content will seek out the path of least resistance.

Creator Jamal Azeez worries that the increasing commercialization of the web will take away the unique culture of expression that has developed:

I'm worried that people will gravitate to it [web series] for the wrong reasons...[Television] companies are trying to jump ship now and basically dominate their own little space in the digital media sphere, and it's not the same thing. It has its own rules, its own audiences, its own expectations, and I'm worried that people are just gonna try and turn the digital media web sphere into what it's been for the past 50 years and that's disconcerting because when YouTube was created it was like this haven, this outlet for very personal, intimate expressions of self, and let's look at YouTube right now; it's a pain in the ass to use....big corporations and studios are just gonna look to take control of [the digital media sphere].

The increasing requirements for creators to make deals with platforms, influencers and brands in order to be noticed are, according many creators, signs of the replication of the status quo on the web:

It used to be like this, the film and television industry was like so difficult to jump into. That was like the big thing, and if you go into web series, you have much more access and much more opportunity, both as like even as a minority actor but also just like as an independent creator. Now it's sort of seeming like they're starting to become both inaccessible, or at least to a certain degree... It's gonna sorta replicate what TV did which is like less opportunity and much more having to have partnerships and having to have networks with certain people. (Independent creator, Yusuf Zine)

Many creators recalled the difficulty of attracting partnerships with online influencers,

as well as MCNs, to increase the reach of their content. Because of the fragmented nature of the web, online channels with dedicated audiences are able to be 
increasingly discriminating, making it more and more difficult for new creators to break through, even on the web.

\section{Discussion and Analysis}

Although Canadian web creators' motives for their entrepreneuring reveal a deep desire to break free from, and to break up the status quo, numerous global systemic and institutional forces beyond their direct control have an impact on their capacity to reach and connect with audiences, tell their stories, and monetize their work. Audiences' expectations for content to be free and ever-flowing, and their willingness to accept substitutions restricts creators' capacities to monetize their work. The particular form of web series- resource intensive, scripted video that emphasizes team work and the traditional Hollywood SoCal crafts of writing, directing, editing and acting, is not a 'natural' fit for platforms like YouTube, that predominantly reward, and indeed have been built around, the NoCal logic of rapid iteration, automation and scale.

Perceptions around the value of online audiences, and assumptions about the supposed 'automatic efficiency' of content made for the web limits budgets and advertising spending. The volatility and opacity of YouTube as a platform and economic ecosystem, and the growing tensions between corporate and creator interests coalesce into an unstable environment in which to tell stories, grow 
audiences, and build businesses. The increasing power of a decreasing number of online platforms centralizes audiences, but also the number of pathways for success. As the respective strength of the surviving hubs continues to grow, the gap between the power retained by the various corporate entities and the creators who populate their platforms continues to widen. This considerable disparity in power makes creators vulnerable to the whims of the hub owners, and the non-transparent nature of their operations.

The irony of a more democratized distribution system is, as this section has demonstrated, that as more individuals contribute to the media, the more difficult it becomes for professional creators to make a living. This is the Participatory Culture Paradox, or shorthand to describe the contradictory set of relations that enables creators' activities in the online space, and at the same time, constrains their capacity to find audiences and monetize their work. As more and more content is uploaded to the web and offered for free, the expectation for free content grows. The Participatory Culture Paradox is a vivid example of the complex process of structuration at work. Agency and structure collide, and constitute each other. Such a view into this process has only been enabled through the combination of the big picture CPE approach, with the more micro-level, focus of entrepreneurship studies. On the one hand, creators and general users employ their agency to contribute to, and post their content to the web. The increasing saturation of online content, however, then leads to the rise of the 
various new structures that permeate the online world and affect creators' capacities to succeed in the ways they hope. This process sets in motion ricocheting effects: because of the sheer volume of content that is available, viewers can and often do opt to substitute rather than to pay for content. Then, as more content is uploaded, the power of platforms that centralize and aggregate viewing and 'make it easy' for audiences grows.

In the past, scarcity existed in the number of spaces available to be viewed. Now, although shelf space is endless, attention is still limited. This restores the kinds of relationships from which creators were seeking refuge: power is, as was previously the case, in the hands of the distributors. The expanded CPE approach developed here lays bare, the effects of the increasing power of media convergence in the online space on creators' agency. Today, a disproportionate level of power rests with the platform owners, who can and do make changes to their underlying systems that have material impacts on the creators whose work populates their platforms with little consideration. These distribution platforms, which on the surface appear open, are designed with specific pre-built logics that favour certain kinds of participation over others. Content is still programmed but the critical and initial part of this process is done at a level that is hidden from view, known only to the platform owners and their developers; the secondary part comes at the level of audience tastes, but this "natural" selection is only possible in the confines of a system that has been highly pre-structured at every 
turn. The unique nature of these platforms is that they develop, with direction and oversight from their corporate owners, micro-economies and hierarchies that install new structures, including new gatekeepers and institutions. On YouTube, these are MCNs, advertisers and brands, and competition for their attention amongst creators is intense and increasing. Thus, although many Canadian web creators' motives for engaging in entrepreneuring derive from their desire for some kind of emancipationoften times to break free from and break up rigidities embedded in the domestic system, the conduit through which they aim to be free (the web), is dominated by extra-national firms that present barriers which are potentially as challenging to their success. By entering the online space, they enter another battle for autonomy and control. In addition, though they seek freedom, by populating online platforms with their work, web creators also add to their growing power. The process of structuration is made visible again, as creators use their agency to escape certain structural dynamics in their domestic environments, and, through their entrepreneuring, add the bricks that contribute to the construction of the edifices of new media. It is clear then, how, "structures are constituted out of human agency, even as they provide the very 'medium' of that constitution" (Mosco, 2009, p.17).

Ironically, as the tension between creativity and commerce deepens with creators being caught in the crosshairs of the corporate policies of the preeminent platform for online video, YouTube, the platform is in danger of losing the inclusivity 
that allowed more authentic, and often 'by-us-for-us' content from the fringes to flourish and find audiences.

While this section described the way global economic and social and cultural forces are shaping the structure of opportunity open to Canadian web creators, the subsequent one shines a light on the peculiarities of the Canadian domestic system, and how creators navigate and are affected by the converged state of affairs. 


\section{Chapter 7 - Barriers and Challenges from Within the Canadian System}

Although web creators have grand motivations to break free from, and disrupt traditional television industry standards of practice and norms, as well as their own positions within the larger, overall system, they face considerable challenges to their agency from a litany of corporate and institutionalized structures along the way. The previous chapter explored extra-Canadian barriers. These various barriers extend from larger, cultural movements and shifts (for example, the Participatory Culture Paradox, or audience expectations of the online space and its content), as well as those emanating from the trans-national, but primarily American-owned corporations that structure the online space that Canadian web creators populate. This chapter explores barriers, both macro and micro, individual and systemic, coming from within the domestic system, including deficiencies amongst creators and their firms.

\section{The Challenges}

\section{i. Skills and Resources}

At the more micro level, many creators felt they lack the kinds of skills and resources needed to position their projects for the best chances of success. Many, in fact, discovered what these key missing skills and resources were in the process of producing their web series. The typology of skills interviewees felt were missing in the 
web creator community ranged from web-specific skills, to more generalized ones related to business development.

One broadcasting executive with ample experience in the online space reflected on what she perceived to be a gap in the knowledge and skillset of Canadian web creators in relation to online audience development:

Another big challenge is that you not only have to think about the content itself, you have to think about the marketing plan as well. And I think there's a major gap in just knowledge. People know how to make a series, but they just don't know how to get it in front of people. They're always just like, 'Well, we posted it on YouTube.' And that's the beginning and the end of the sentence. And you're like, 'But there's no content plan to support it editorially across different social media, or how do you engage or respond to audiences when they do watch your series?'...[T]hey don't know how to create that momentum. So that's a major problem. Just a knowledge gap...understanding... (Broadcasting executive)

Jay Bennett, Senior Vice President, Creative and Innovation at Smokebomb, agreed with this assessment, noting also that growing online audiences requires a skillset that is different from growing audiences for traditional formats:

It involves speaking to them in their language...Don't just take your poster for your show and start dropping it in groups. Go and understand how they talk and communicate and what they love, and actually reinvent your poster and your messaging and your language to fit that. And if you have to do that five different ways, do it five different ways... You have to bring them back one at a time. There is no such thing as, 'If you build it, they will come'. There isn't. (Jay Bennett, Independent production company executive)

More experienced creators often acknowledged the need to use social media platforms to create ongoing points of engagement for audiences: 
YouTube's a very crowded space, and if you don't have...the social media engine behind it [that's a problem] ...you have to continue it [your story] through and you have to plan that as part of your marketing strategy, and part of your release strategy, and part of your next production-cycle strategy...I think what happens is... unfortunately, we're often busy making it and not really thinking about longterm or long-tail promotion of it, the content...And you do need quite a while to get the attention to move up, to really get the prestige, get new eyeballs, all these things, they take long-term, but you need to have extra content to grease the wheels of social...You have to have a real engine driving and building that audience... (Independent production company executive)

While creators across career stages acknowledged the importance of social media skills for audience development, many admitted to not having the particular set of skills required to do this work. One early stage creator commented:

I think maybe at the time we were very new to making web series. There's so much more research and so much more you have to know about that industry to really go into it, and I think we just weren't fully literate on all of that... how do you create a fan base, how do you reach that audience? (Yusuf Zine, Independent creator)

Another early stage creator reflected,

I tried, but what I found was the biggest stumbling block was reaching out to audiences and getting traction, or even just getting [the show] out there... just didn't know, and I still don't know where to begin. I did what I thought you would do, and I assumed... I had people post on Reddit and that kind of stuff, I'm like 'Oh, you put it on Reddit and somebody finds it, and then that's it. That's all you do. That's how things work.'... No idea. (Alex Young, Independent creator)

A more established creator believes his lack of online audience development skills was

a chief barrier to his series' further penetration and success:

I was making it for me. I loved this stuff. I figured if I love it, there must be other people that love it too and I just kind of made the story that I wanted to make, figuring it would find its own audience and that was maybe part of my downfall, which I knew why it didn't become more successful...[T]hat's not something I'm 
really good at-identifying the audience and how to reach them and target them. (Independent creator)

The frustration, for many web creators is that because of the lack of supports and resources available, they are required to be experts in a wide range of disparate skills. Digital creators must know "everything about tax credits, both digital and traditional...everything about production...distribution...Financing, social, casting, script, story, production values, special effects, post-production, camera..." (Independent production company executive).

Another area that creators identified as a weakness, but one which has been recognized as an opportunity by leading firms, is the utilization of big data, and data analytics in the process of program planning and development. Netflix, for example, is known to utilize big data, and data analytics in the crafting of its programming. Its first original series, "House of Cards" was strategically developed this way (Carr, 2013). Although independent web creators do not have access to the kind of big data Netflix does, they often have access to some data analytics from their distribution platforms regarding demographics, audience comments, time watched, and viewing patterns (when those watching tune out, for example). One creator admitted that he simply "didn't understand how to implement or analyze analytics enough to utilize that as a tool" to inform series development. Others that showed an interest in utilizing data analytics acknowledged that while it could have been useful to use data to inform 
production, web series are often fully scripted and fully filmed before the first episode is distributed. Making changes based on audience data would thus be costly and would require rewrites or re-shoots. Such changes, therefore, are challenging to accommodate with limited budgets.

Another creator who is a founder of an emerging production company with a history of success in selling short-form web series said that even with more resources available to him than a sole proprietor, he was unable to exploit some of the affordances of the digital space, including his direct connection to, and insights about, his audience "as much as we should" despite knowing that some have found "great success by just looking at who their audience is, where their audience is, and targeting them" (Independent production company executive). For his firm, the need to "do everything" prevented his team from being able to harness the benefits of digital distribution in ways that could have potentially amplified the success of his series in the area of audience engagement. Other digital skills creators identified lacking is optimization of content for the web (search engine optimization, for example), coding, and website design.

Many of the creators interviewed also expressed a belief in their lack of general business acumen, commenting on perceived tensions between being a "businessman" and an "artist", or an "artist" and an "entrepreneur".

One creator reflected, 
To be completely frank, ultimately, if I had to say, "Am I a businessman or am I an artist?" I'm an artist. I wanna find business reasons that I'm allowed to make the art, rather than find art that will allow me to make a business move. That is ultimately how my brain works, for better or worse...(Independent Creator)

Another creator expressed his desire to focus on the creative elements of his work, rather than the business ones. He admitted that although he knew untapped business opportunities such as merchandising exist given his series' large audience size, he did not view himself as qualified to exploit them, and, in fact, preferred not to:

I am [an entrepreneur], but I'm not a very good one. I would rather someone with the business training do that for me. 'Cause I don't wanna divide my time with it. It doesn't interest me. Making money interests me, but I don't wanna do the work. I wanna do the work that means doing the creative, 'cause that I can do and I am skilled at that. My skillset isn't in T-shirt distribution. (Independent creator)

One former broadcasting executive, and the current founder of Tablerock Media, a company about to launch an over-the-top service with programming catering to the niche interests of aviation, motorcycles and guitars, expressed his belief that many Canadian producers do not possess the entrepreneurial skills necessary to thrive in a rapidly changing media landscape:

The problem that we've got right now, it's happening everywhere, I see with producers all over this country-there is no work. And so... most of these people are...entrepreneurs, but they're not entrepreneurs in the sense of, 'Well, we're gonna go out, we're gonna get money, we're gonna fund this. We're gonna build a new platform or new infrastructure to do this.'... They're really good storytellers...(Jeffrey Elliot, Co-founder, Digital production and distribution company) 
Carly McGowan, an executive at the IPF, noted that Canada's media production firms tend to think on a per project basis, rather than on a firm basis, and are not used to taking complete ownership of their intellectual property:

The way this whole system is built, even with digital media companies, you build something and you hand it off, and then you move on to your next project and you build it and then you hand it off. But they're not really... 'This is our baby.'

According to Andra Sheffer, the CEO of the fund, it is also the lack of financial resources to hire individuals with the right skills that poses a significant impediment to the success of many web series, particularly in the area of audience development:

The problem is you launch, you put a lot of energy into promoting your launch. You stick with it during the run of the first season and then it all starts to fade away, and no one has the money to pay for a community manager or a social media person... They don't have the skills...And they don't have money to pay for the labor to keep it all up...[The ideal digital creator]... is the modern producer who is also a publicist in a way; they have to be actively involved in the promotion, and they're not used to that... (Andra Sheffer, CEO, Independent Production Fund)

One established web series creator noted that on many occasions, he has found that social media and digital audience building skills are assumed by distribution partners, which often expect creators to promote their own work:

We would get all these platforms and say, 'Hey, we want you to put [our show] on your platform.' And they'd be like, 'Great.' And we'd say, 'So what are you gonna do?' They'll be like, 'Oh no, that's up to you.' It's like, 'Well, we don't have the resources or the skills or the time...You're the distribution partner. You're the one who's supposed to push it'. And nobody wants to be that person anymore... (Independent creator) 
Recognizing his limitations, one creator earmarked some of his scarce resources for the hiring of a social media expert. The expert, however, had never worked in the promotion of online independent series, and "did a horrible job" since he "was doing things that were for different mediums", and despite being "successful at what he does [in his industry]...it didn't help us at all..." (Independent creator). According to the IPF's Andra Sheffer, a significant challenge even for the web series creators who receive funding is that although they have some money to spend to hire for audience development, "there aren't enough good people out there who even specialize in doing this".

The deficiencies in skills, then, are germane at both the individual and firm levels. Without the resources to hire out, and an apparent dearth of existing professionals with the specific skills required even when the resources to hire are available, web series are not best positioned for success.

\section{ii. Working with Broadcasters}

As independent web series creators look for distribution partners and extra capital to fund their productions — they often approach Canadian broadcasters in search of deals. Throughout the interviews, web creators who have managed to secure deals with the majors reported challenging experiences due largely in part to what they 
believe is the majors' lack of understanding of the internet as a distribution channel for programming.

Several creators reported their series being used to anchor various broadcasters' new online efforts (on proprietary websites or on YouTube), only for the channels or proprietary online portals to be neglected, or, as the creators perceive it, abandoned.

As an executive at one production firm recalled,

We thought that they had a plan to launch other content and make it into a little something more than what it was, but they never followed up. Had they launched another series, maybe people who went for that series, would then watch ours and it would've helped keep that longevity going of eyeballs going... If we launched on an established channel, we might have had a little bit more success in terms of how many people watched. (Independent production company executive)

Another creator described his experience:

They launched us on [the broadcaster's website], and then now they're gonna put us on YouTube without taking it off [their site]. Plus who's gonna watch it on YouTube when you could've watched it on [the broadcaster's site]?... They didn't give a shit about us. They didn't even know. We totally created something in a hole and then we were like, 'Well, we made it.' Why? Because honestly, I don't think they cared about the content. I think they just wanted to have an online series. They literally were like, 'We want an online series. I don't really care what it is.' ... I think they don't know that they're killing it, because they're so unaware of how web works. They're like, 'No, no, no, this will be great! We'll put the pilot on every single thing.' And then I'm like, 'Oh, good. So no one will know where to see it'... They don't understand that putting two commercials in front of your thing [is not a good strategy], and you're like, 'Great, no one's gonna watch it. Awesome.' I don't think they understand how detrimental it is...(Independent creator)

Another creator who was hired to support the promotional efforts on a web series that was being made in conjunction with a broadcaster was frustrated that the 
broadcaster appeared to have made little progress in its understanding of the web

since he had last worked with it a decade ago on his own series:

[l]t's disgusting that we haven't moved in 10 years... They want to still put it on their website. They don't know what to do with YouTube. They're not doing YouTube correctly. They're not doing Twitter correctly, the Twitter account is dead, it's not moving. All of that stuff. Now, you could argue that's 'cause [that broadcaster] is going down the tubes and the rest of Canadian television is going down the tubes too. But whatever the excuse is, we have not gained anywhere... They don't get it. Or they don't want to get it because things are so damn good for them right now. (Independent creator)

One established digital creator compared the online efforts of the American-based

Comedy Central channel to those of the Canadian Comedy Network:

So, Comedy Central in the States is pretty much the equivalent of Comedy Network in Canada, I would say, in terms of what their brand is. They're the comedy channel. And the US has 10 times our population, roughly. You would expect our viewership tends to be around one-tenth on TV of what the viewership is in the States. There's roughly a one-tenth market size difference here. So, I would expect most numbers, unless there's a compelling reason otherwise, to be one-tenth. Now, when I first started talking about this two years ago, Comedy Central was going crazy building their YouTube platform....and they were just blowing past one million subscribers... They were building up their platform. They were putting promos out... And I would ask people, 'What do you think Comedy Network's YouTube channel is like? How many subscribers?' One-tenth maybe, maybe 100,000 subscribers... And at the time the answer was $32 \ldots$ (Independent creator)

Currently, the Canadian Comedy Network has 944 subscribers on YouTube. Like many others interviewed, this creator believed the Canadian network displayed an immense lack of care with respect to its online efforts:

So they have a bunch of videos where the audio was in the left channel only, right?... That's how much they care about it. But they tweet to it...There's really no traffic... And the people working there know there's no traffic and they don't care. It 
really blows my mind...So they haven't really built anything online. (Independent creator)

According to many interviewees, Canadian broadcasters' generally lacklustre online channels are especially problematic when it comes to seeking partners for distribution. Applicants to the IPF, for example, are encouraged to secure commitments with strong online channels [IPF, n.d.] to increase their odds of securing funding since platforms with existing audiences in the same target demographic strengthen a web series' chances of finding audiences. One established digital creator characterized the situation as a missed opportunity:

Working with a broadcaster is like putting your web series in the witness relocation program... No Canadian broadcaster is taking the online spirit seriously...[T]here's a bunch of IPF web series out there, every year that are looking and shopping around and trying to get a YouTube channel to distribute them as part of their strategy for the application. So they're looking for million-plus subscription channels...There's no one in Canada that has that. [l]t's a real shame that we have to go outside of our borders. (Independent creator)

Several creators interviewed admitted to seeking distribution through US-based online channels, over Canadian ones, for this reason.

\section{iii. Broadcaster Digital Leadership}

Many creators, and indeed, some of the broadcasting executives who were interviewed, suggested that the country's broadcasters lack the kind of leadership and forward-thinking, at the highest organizational levels, required to exploit the new opportunities enabled by internet-based distribution, and have too many conflicting 
assets, including cable, to effectively do so. Former broadcasting executive, and current co-founder of a digital startup, Jeffrey Elliot, argued that the constant need to appease shareholders impedes long-term thinking: "[T]hey're public companies, so they're focused on the next quarter and making their year... It has nothing to do with where the business is gonna be five years down the road. It's very short-term thinking...". Another broadcasting executive commented that investments into the online space should be seen as investments into the future, as they are in the US:

Our people aren't making decisions.... don't know if there is any profit [in online content distribution]...I think Americans are thinking about this as an investment to the future of where the audience is. So, I think whether or not it's a loss or not, I think it's a necessity...it's just like you need to play in that space. And you look at Amazon, case and point. I guarantee you they're making no money and even, they publish multiple reports that they're debt financed... (Broadcasting executive)

Many creators also felt that broadcasters are generally reluctant to take on digital

projects and do not take them seriously:

When the bigger production companies and the broadcasters get into it, they don't take the people who've been out there, experimenting and learning and building that reputation, they go for kids who have no experience, they go outside the country, they take people out of other jobs and put them into it, they give it the lowest possible budget... Over and over, they've said, 'I don't wanna do it. It's stupid. It's like a waste of money. I put my youngest people on it. I don't pay attention to it,' instead of taking the leg up that would put them ahead of the rest of the industry. And so, this is really our chance. This is the first time a little country can take a little bit of money and hit a worldwide audience and make an impact and the window is closing on that. (Independent producer) 
Another executive working at the majors noted that there is often wastage of talent that occurs within broadcasters due to rigid hierarchies, which are sometimes dictated by union rules:

I know a lot of talented digital producers that have worked in broadcasters, and they're either the first to be let go...or they're not senior enough to get the next promotion...So, you know you're bottlenecked by the person that might not have the experience, but might not have gotten surplussed from another team...You don't have the right leadership. (Broadcasting executive)

Another major problem, according to the executive, is a lack of interest on the part of leadership in learning new skills and understanding the distinct peculiarities of the web as a distribution method:

I really do think that part of it is the skill gap. Risk-aversion is one thing, but risk aversion, I think, is most the time a substitute to say that they're not capable of understanding it... It's like, 'We don't wanna learn it, so I'm scared of it.' I think that's the reason we're not moving forward, is that people making decisions are not just scared, but they aren't eager to learn it. Huge problem. Huge problem. (Broadcasting executive)

Other interviewees lamented about what they viewed as confusion within broadcasters' online strategies. One established digital producer could not understand how one broadcaster's three online portals were intended to work together: "They've got Crave, they've got CTV Go, and they have Extend, so what exactly are they doing? ... You bury CTV Extend...it's impossible to find. What are they doing here?" (Independent production company executive). Another commented, 
I think CTV Extend...shot themselves in the foot. They went and built this whole platform, and they launched two shows. What they should have done is they should have launched two shows every two weeks for three years. If you actually wanna do it, actually do it. It's not rocket science. It sounds like a big number, it's not. Take 10 million bucks....Three-year plan, you'll actually build something. (Independent production company executive)

Bell Media has shuttered the CTV Extend online channel since the interviews took place. Thus, in addition to an apparent insufficient understanding of the web space, many creators also believed broadcasters' online efforts demonstrated a lack of genuine commitment to growing strong online businesses and brands. One established digital creator compared what the US YouTube channel Geek and Sundry was able to accomplish with the million dollars in seed funding it was given by Google:

Geek and Sundry...so they got a million dollars, and they turned that into a million subscribers...[T]hey did it. A team... They took a brand from scratch and within a year, turned a million dollars into a million subscribers. No broadcaster in Canada can do that, even if they allocated a million dollars. (Independent creator)

Geek and Sundry currently has greater than 1.5 million subscribers.

Many interviewees were also generally confused by the strategy behind Canada's broadcaster-owned OTT platforms, Crave (Bell) and Shomi (Rogers and Shaw; now defunct), which only operate in the Canadian market. On the topic, a former broadcasting executive commented, "If the estimates are true that Rogers and those guys each spent $\$ 150$ million...I don't know what the end game is 'cause they're gonna get just smoked by international competition...the second $\mathrm{HBO}$ is available in Canada". According to him, "as an industry", we are still "thinking very much like a 
colony. And we'll just look after Canada, like that's all we have to look after". He

believes the majors should be thinking globally.

Several creators were also frustrated by reportedly being asked to provide their web series for no payment to the CBC's Punchline platform, an online comedy channel, now also defunct:

We talked to them [CBC Punchline] when they first happened, and they basically asked every IPF web series to have their show for free for good faith and good will and maybe working together in the future, and to get more eyeballs. [At first] we sat down with them. They're like, 'We love [your show]! Love your baby, love your baby.' (Independent creator)

According to the creator, the broadcaster indicated it had only a nominal budget for content, and could not pay for his series, which he thought was highly problematic:

I remember leaving that meeting and thinking, 'Well, if you guys know how to get a million subscribers and build up a significant online presence without paying for content, then that's a magic trick I'm not familiar with, and I'll watch and see how you do it.' (Independent producer)

None of the creators interviewed whose series appeared on the Punchline platform were paid for their content. According to those interviewed, the CBC's practice was to remunerate only CBC Punchline originals. Such a practice is commonplace on the web, and creators often provide their content (for free) in exchange for access to larger audiences. Prior to it being shut down, the Punchline platform did not appear to be well received by the Canadian public. One media observer described the platform as a "strange mix of original content and a sprinkling of clips from 'This Hour Has 22 
Minutes' and other CBC comedy shows" that "seems to lack any clear purpose or audience" with a website that is "utterly incoherent" (Daro, 2014). CBC executive Sally Catto reportedly called the platform a poorly researched "branding mistake" at a recent industry conference (Ashton, 2017; Playback, 2017). CBC appears to currently be commissioning more original digital series for its new online portal, $C B C$ Comedy.

iv. Funding

The funding system, both public and private, was also found to be a source of frustration for many creators. Prior to 2017, the CMF, the country's primary source of funding for television series, did not fund any linear (non-interactive) series without a broadcast trigger license. At the time the interviews took place, the private IPF was the key production fund for independently produced online scripted series across Canada. Web series were also ineligible for federal tax credits at the time. There have been some advancements to public funding at the national level, as well as to the federal tax credit program. These various funding sources will be discussed in more depth in the subsequent chapter on policy and program recommendations.

At the national level, the Canada Media Fund requires all broadcasters receiving funding for television to include a "Digital Media Component" that is directly related to the television production for which funding has been allocated (CMF, 2017a). It is in this sense that the CMF requires television projects to be "convergent" (CMF, 2017a); 
a television project thus falls under the CMF's "Convergent Stream". The Digital

Component can take a variety of forms and can be a website, application, or linear and interactive content that is connected to the television property. Depending on whether the Digital Component is considered "value added" or "rich and substantive", it must have a budget of up to, or over $\$ 100000$ respectively, for English language projects (CMF, 2017a). In some cases, the Digital Component has taken the form of a scripted web series based on the intellectual property of the funded project. For example, the Canadian television series "Murdoch Mysteries" has a web series entitled "Beyond Time" that fulfilled this requirement and follows the stories of supporting characters in short, two-minute episodes.

Many of the creators interviewed felt that the money devoted to the required Digital Component of CMF funded television projects could be better spent. One broadcasting executive at the majors believes the Convergent Stream disincentives broadcasters from thinking more deeply about the role and purpose of online content:

Digital is a second partner... It's a second window or it's a second tag... It's a convergent stream, it's that extra piece that you have to do, versus, is it part of the thinking in the ground level of what you're building? So is there consideration of what digital means? How is that social or digital piece or pieces going to go into ' $x$ ' show that you're commissioning? (Broadcasting executive)

The executive argued that thinking about digital content must "start on that ground level", but rarely does. 
One established creator was extremely frustrated by what he viewed as a

significant waste of funds through the Convergent Stream:

So the CMF convergent fund... for television series... to make an online component. Now, I can tell you, anybody that has a television show does not [care] about you. It's an afterthought. It's like, 'Oh, I can grab some producer fees from this. Let's just make whatever videos, I don't care'. When you have a television series, you don't [care] about making webisodes. Right? So why is all this CMF money going to that? I can tell you, when you're making a web series and nothing else, you...[care] about your online content. So, that is a failure in the system, I think. And it's a failure because there is no online broadcast infrastructure, a complete lack of monetization of online content. (Independent creator)

If concerted efforts had been made to build online broadcasting infrastructure that would allow for the monetization of online content, the creator felt broadcasters would take their Digital Components more seriously. Another broadcasting executive working at the majors observed that although "a lot of money has been spent over the last decade on digital extensions to shows, web series..." under the Convergent Stream, there are few "best case scenarios". One established creator suggested that the priority should be "...having more shows out there" rather than "having an app that goes along with that show that you're never gonna access" (Independent creator). Currently, all of the substantive production funding programs for web series in Canada have been modelled after traditional television funding programs, which were built around large infusions of capital for projects that are fully developed. The funding programs for which web series are eligible accept applications at a maximum of twice 
per year. IWCC Vice President Dan Speerin believes the protracted funding cycle does not work for the amplified pace of the web:

[T]he funding model doesn't work for the Internet. Because if you give me 20 grand, it's amazing, except I don't have the ability to wait. I gotta put content up tomorrow. I'm on the Internet....[Y]ou make a show, so you make six episodes...10 episodes. That's two months of content and now you're in a funding cycle, waiting for IPF 2, which isn't until a year away. So it's great if you're making a calling card. If you're trying to be sustainable on the web you better be really creative with your accounting, otherwise it doesn't really work. Because after six weeks, 'What are you doing now for me?' It's the web culture....the Internet moves way faster... it doesn't work the same way. (Dan Speerin, IWCC Vice President)

Interviewees had issues not only with the length of funding cycles, but also with the way funding is allocated:

I think there needs to be more of a thinking of instead of again, tent-poling content, so it's not just like, 'Okay. Here is enough money to create five episodes.' And it takes you two years to close the financing on it, versus thinking of longer term, incubator-type processes that allow you to create...that iterative, like lean startup thinking? Like, create your minimum viable product, get feedback. That's what YouTube is, right? ... 'Okay. Do they like this tone? Okay. No, next. Let's try this format. Let's try that'. We don't have that type of thinking in our funding process. It needs to be fully baked and by then, by the time it's out the door, you've already invested 200 grand in there or something. And it's too late to change and/or if they like it, then you're also too late to make more. So I think we just have to stop throwing big chunks of pizza at the wall. (Broadcasting executive)

Another creator also agreed that to increase odds of success, funders should invest in more content, even if this means investing "less in each show" (Independent creator).

Prior to the introduction of the CMF's new pilot funding program for web series in 2017, digital-first creators wanting to make any series without a broadcast trigger 
licence, could only apply to a fund aimed at "interactive" content. This was challenging because, as one established creator observed,

[S]cripted [online content] doesn't fit in reinventing the wheel in technology pieces. We've definitely done our best to insert it whenever possible...And not all stories lend themselves to a digital experience. Sometimes, it's a square peg in a round hole... (Independent production company executive)

Creators admitted to tailoring their content to meet the "interactive" requirements, even if such additions were not necessarily best for their projects.

In addition to the lack of appropriate funding models designed for web-based content, creators also found it troubling that web series were ineligible for federal tax programs. Creators could not understand "why, if you're employing people and you're paying them in this country, why you wouldn't be eligible for a tax credit" as is the case with film and television (Independent production company executive).

As much as most creators believed that improvements in funding programs were required, many also expressed a worry that web creators would become overly reliant on the funding system, as is the case with traditional television. As one established digital creator explained,

[G]etting this fund, that fund, this fund... makes the thing that matters engaging with the funding agencies, but my theory, my idea is the reason why Canadian movies and TV shows don't connect with a wider audience is because the content itself is irrelevant to the process. Nobody gets paid depending on whether or not anyone likes it...I think taking the web series spirit and trying to connect to the traditional Canadian funding models, I think there's a danger there in chasing the same dragon. Being on that treadmill of like, 'Oh, we made it.' Get it online and it 
doesn't really matter in the long run if anyone sees it or anyone likes it... (Independent creator)

The concern for many interviewed was that the reliance on funding could lead to the same lack of innovation and competitiveness that has been observed in the traditional television sector. Others also worried about the introduction of more gatekeepers to a world that was previously more open. Many reported observing a clear increase in the expectations of web creators applying to the IPF, for example. One creator commented,

And it's becoming a situation where it's very gatekeepered, because then also the people who are applying for these IPF funds, a lot of times are established production people. And so, all of a sudden, it's like you have someone who's an independent web creator who has been making content for let's say 10 years, but they don't have the big guns. They don't have...someone big that's gonna be on their show. Which, again, creates some difficulty in terms of being able to acquire funding. (Independent creator)

Interviewees were therefore worried that heightened expectations could lead to the exclusion of new voices to the Canadian screen system, as well as act as an impediment to diversity in both content and creators. The executives interviewed from the IPF, in response, indicated that there has been an observable increase in the quality of submissions the fund has received from its inception to the current time, and that the fund also adjusts its expectations as they learn more about what makes online series more likely to succeed. 
Finally, many creators also believed that the various web series funds that are available have "financial expectations...not in line with the realities" (Independent web creator). The CMF's new pilot program for web series, for example, for which the researcher acted as an inaugural expert judge, expects $100 \%$ recoupment as well as profit sharing (CMF, 2017b). Web creators are expected to report until recoupment is complete, and several felt the expectations were too onerous for "people in a garage" (Independent web series creator).

\section{v. Hiring Canadian Actors}

Throughout the interviews, creators also frequently identified challenges related to the hiring of union performers i.e. members of the Alliance of Canadian Cinema, Television and Radio Artists (ACTRA). Prior to 2016, ACTRA did not have any formal agreement for internet-based productions, and required negotiation on a case-by-case basis. The typical deal, according to most creators interviewed, was a 35\% discount on standard actor rates in place at the time, which would amount to $\$ 434.69$ per performer, per day (ACTRA, 2013). This rate did not include overtime or residual payments. At the time of the interviews, many creators were already aware of the terms of the new agreement, which went into effect in 2016, and expires in 2018. The $35 \%$ discount on standard rates was formalized in the new agreement for productions with budgets of up to $\$ 7500$ per minute (ACTRA, 2016). 
By far, the most significant issue creators reported in relation to working with ACTRA was the cost of hiring unionized actors, which they found to be prohibitive. This concern was widespread and had impacts on both first and subsequent season productions.

Many creators reported that using union actors amounted to $60 \%$ of their total production budgets or more. This, according to the creators, had material impacts on the rest of the production departments, often resulting in reduced production quality, reduced pay for other crew positions, inability to pay overtime, and producers deciding to forgo their own pay.

Many indicated having a strong interest in hiring union actors due to their perceived value. According to one production company executive, actors "are maybe the most important, apart from your creative, and their face carries the project" (Independent production company executive). However, after not being able to negotiate feasible performer payment rates, many reported having to change their casting strategies. One established production company executive was intensely frustrated by his experience: "My cast budget now is $75 \%$ and I can't make it now. I have to go non-union" (Principal, Digital production company). An emerging creator commented, "[W]e'd love to, we weren't against the idea of working with ACTRA and we still aren't, but it just didn't...make sense" (Independent creator). 
Many interviewees believed that ACTRA's policies were out of line with the realities of the digital world. They believed the union's rigid approach comes from its fundamental misunderstanding of the digital production economy. One funding executive remarked,

The problem I think with ACTRA is, they don't understand how this actually works. And they think that producers are running off making millions of dollars... It's a big, big issue... Is Joe Blow [who is] doing his first thing, and trying to make a track record [making millions]? (Funding executive)

Many creators emphasized that they are not attempting to exploit actors, but are simply dealing with the realities of small budgets:

[A]s a web series creator, you're working with no money...And I understand the need for performers to certainly protect their rights and to protect their abilities to make money. But I think that there comes a point in time where those performers want to chase exciting opportunities for promotion, and those creators are not looking to take advantage of those performers. They themselves are merely working with the tools that they have. (Independent creator)

For many creators, the situation often became acute when one of their principal actors became union in between seasons. In order to avoid the extra production costs, many creators would find ways to write these characters off. One creator explained the difficult predicament he was in when this occurred:

[W]e had troubles midway through our production, which was that one of our actors, thankfully, became more successful... And so as a result, this person joined ACTRA. The problem is, we had to write that person out of our script. And the actor didn't wanna do it, the actor didn't wanna stop working on it, and we didn't want to get rid of them. (Independent production company executive) 
Another creator commented on his recent experience with the same issue, "I've just decided... that character died" and replaced him with another non-union performer. Others recalled having to turn away more established actors because of their union status:

It's been kind of challenging because we've received some interested enquiries from union actors, and I've had to kind of say, 'Well, we're not union.' It's way more than our budget will generally allow for us. (Independent creator)

One established digital creator described the situation as a "game of whack-a-mole where you either have to graduate to ACTRA rates, or your show dies" (Independent creator).

Creators also emphasized what they viewed as the danger of pricing union actors outside the reach of emerging creators:

They're hindering a lot of creative things right now. Like we're trying to work with other people in different capacities. As soon as they say they're ACTRA, it's like a non-starter. Conversation ends...[T]hat's a major, major problem...that's stifling a lot of digital growth, in my opinion, right now. (Independent production company executive)

According to creators, the premature ending of the conversation has a three-fold impact on the Canadian digital-first industry. Firstly, creators noted that emerging and promising Canadian performers, who have very little opportunity in the Canadian market, are prohibited from participating in more meaningful work:

[ACTRA] also have to be cognizant of the fact that there's not a lot of work in this city for [actors]...especially for TV and film roles for Canadians in Toronto... You're looking at going on these auditions where they see hundreds of people and it's for 
basically two lines in one scene, whereas in web series actors are getting the opportunity to be the lead and have something actual to do. (Independent creator)

Creators felt that actors are thus missing out on opportunities to showcase themselves in roles they have never been offered. It should be noted that ACTRA does have provisions for performers who choose to work with other performers in a "co-op deal" in which the rights and profits of a production are shared equally between team members and two of three of the writer, producer, and director must be ACTRA members (ACTRA, n.d.). This kind of agreement will, therefore, not work in most situations.

Secondly, creators emphasized the limiting impact pricing union actors has on the scope and scale of Canadian web series production:

I'm always gonna go ACTRA. I'll just suck it up. I won't get paid. I won't get paid and I'll use ACTRA actors because I understand the value of them and that's a sacrifice I'm gonna make as a producer... I'll do it for free, but the fact is that if I was able to get paid to do this, I'd probably be developing more shows, and I'd be putting more actors to work. (Independent creators)

Since some creators opt to hire union actors by pushing past financially responsible limits to enhance their chances of securing the best performances possible, they are unable to grow their firms and take on other projects in competitive timeframes, resulting in longer periods in between productions, and therefore, less ongoing industry work, for actors and those working in other roles. 
According to interviewees, the third impact that pricing union performers out of the reach of digital creators has on the digital production ecosystem is that it impedes the development of centres of excellence, as well as advanced skills:

My biggest fear is that by putting the best actors in our country... out of reach of young filmmakers or creators, then how are we training our young filmmakers to become better if they're working with less than stellar talent? It's a huge difference as a director when you are working with professionals who know what they're doing just purely from the perspective [that] one film does not make a filmmaker. A director is developed over years and years and decades possibly...So if you're giving them terrible resources, then they're never gonna get better and they're never gonna catch the eye, and they're never gonna grow and help develop more content and so I think that it seems like everybody in the business gets that for the most part, and is trying their best to support these content creators, who are mostly writer/directors in terms of helping them along...Everyone wants to protect themselves, and I get it. But if you wanna make the industry flourish... Those opportunities, when you get a chance to practice your craft, and work with people and build a community, we shouldn't be denying people that. (Independent production company executive)

In addition to impeding industry advancement in the ways described, one creator also noted that although she was passionately committed to casting diverse principal talent, ACTRA's diversity policies often place diverse talent beyond the capacity of web series creators:

One of the big difficulties about casting diversely in independent web series is ACTRA's rules for diverse candidates are more relaxed to get into ACTRA. So, diverse actors tend to get into ACTRA quicker...[S]o you basically have to get them and finish with them before you lose them. (Independent creator)

According to the creator, the union's policy on diverse talent has the unintended effect of making diverse casting risky from a strategic perspective for web creators. 
Creators also reported frustration with what they viewed as an opaque system of negotiation with the union:

We spoke to ACTRA at length about it, and we weren't able to find a way to work together...Some of my earliest projects, the largest challenges I faced was working with ACTRA on them...[W]e heard about many, many side deals that have happened, and that all of them were under wraps and we were not able to look at the terms of those deals...[T]he problem is that I don't have a really strong connection with the ACTRA executive and so I don't know what they would be willing to do... (Independent production company executive)

Several also reported attempting to negotiate with the union, only to later find out that other productions managed to secure more desirable rates. Many felt that those who had existing relationships with the union were generally able to secure better agreement terms than those that did not. The new standardized agreement will put an end to such situations.

Of the main Canadian guilds and unions, creators felt that ACTRA had the most rigid agreement terms. One established production company executive compared his experience working with the Writers Guild of Canada (WGC):

[W]e also use the WGC...[T]he WGC is much more flexible, and they're willing to negotiate, and they understand the realities of producing for digital, and that there are certain circumstances where you just don't have the money.

The WGC has no set rates for writers working on new media productions (WGC, 2015).

Several creators noted that the issue of being unable to hire union performers is so critical from their standpoint that they have shifted plans from filming in Canada, to filming in the United States (Los Angeles). The US-based performer union, the Screen 
Actor's Guild - American Federation of Television and Radio Artists (SAG-AFTRA), has

more flexible new media agreement terms. Until a production reaches a budget of 50

000 USD per episode, performer rates are fully negotiable between the performers and

producers (SAG-AFTRA, personal communication, email, May 16, 2017). Very few

independently produced Canadian web series have even come close to this budget

threshold.

One established web series creator explained why he believes the SAG-AFTRA

agreement is superior to that of ACTRA:

It's freedom, it's power to the actor. It's kind of what I think a guild or a union should be for-to protect the actor when they need protection, but not protect them in that sense of, 'We'll tell you when you need protection.' And the SAG actors on the new media productions, are allowed if they wish, to negotiate their own salary and choose to work for free. So on a SAG new media production, we could pay our celebrity a couple of thousand bucks and get actors who are like, 'I would love to get that more real material with this celebrity, and yes, I will work for free.'... You can set a model that works for your project... (Independent web series creator)

For his own project, he was unable to pay both the Canadian union principal performer rates and also secure a celebrity actor so he decided to move filming to Los Angeles. He remarked, "[Y]ou can't afford to do this under the union here. I'm gonna have to go to LA and do it there, and Canadian crew lose jobs. And Canadian actors are losing acting opportunities" (Independent creator). Another creator remarked,

I'm a big freedom-of-choice proponent. I always say 'If an actor wants to be able to do this, why are you telling them that they can't?' I honestly think the quality of web 
series would go up significantly if ACTRA performers were able to act in small web series voluntarily without...getting [the standard] day rate.

Interviewees across career stages and affiliations firmly believed that ACTRA's policies require adjustment, and that as currently formulated, present a barrier to web series industry advancement.

vi. Accessing US online buyers

Canadian creators also reported challenges attracting high-destination US-based streaming services as buyers. Interviewees working as independents, and as part of established and emerging firms indicated that Netflix, Amazon and Hulu were difficult to access.

One established creator commented that to connect with one of the top USbased digital streamers,

You have to actually have their personal e-mails...[T]his is why one would go to a ...media festival ... because you have to meet them in person in order to get their emails in order to contact them in order to pitch. If you do not know them in person, it's very hard. (Independent creator)

For most independent creators, or even independent production firms, the requirement to physically travel to the major screen markets and festivals is cost prohibitive. As one production company executive noted, in a small firm, "you have very little money to travel, and to attend events, and to attend markets, and to get through to a place to bump into them" (Independent production company executive). 
The reason for the increased distance between producers and the streamers, is, according to one established creator, two-fold. Firstly, such distance acts as a tactic to curb and manage an oversupply of content and interest, and secondly, it stems from a specific operational culture and context that is fundamentally different from traditional broadcasters:

It's very hard to set up a pitch at Netflix, Amazon, or Hulu. It's much easier to set up a pitch at a traditional network. You'll get rejected, but they are much more open to creators than these over-the-top networks. I think the over-the-top networks just get inundated with more stuff. And also they come from a different culture. They don't come from a creative culture, they come from an analytical culture. So, if you're dealing with Amazon creatively, they are not in their corporate culture, allowed to say, and I'm not making this up, this is from personal experience. They're not allowed to say, 'I like this. I feel...' They have to back up everything they say with analytics... Decisions in traditional networks are still based largely on gut and on focus groups and on random choice. With the OTTs, they have the ability to look at their algorithms, to look at what people are watching, and they can in theory, pick series that are geared more towards that. (Independent creator)

Another established production company executive also commented that the culture at these streamers is more opaque than what most Canadian producers are used to in the domestic system:

I had meetings with Netflix and Amazon and they have both been kind of first contact meetings which usually is like they are at a conference or something and I catch their ear and it's very, very difficult to get past that gatekeeper to a point where you can actually have a conversation of value... I am stunned by how [opaque it is]...but it's culture shock and the only reason that we're shocked it's so opaque, is that all of us live in an environment where ....all of the major sources of funding for production in Canada are built on systems that are egalitarian, and what you learn is that there's nothing about those private companies that means they receive any bonus to be fair. (Independent digital production company executive) 
According to the creator, there is no incentive for these firms to be more transparent.

For Canadian companies, the difficulty to capture the attention of the US-based streamers also comes from an inability to compete with established Hollywood competition:

Netflix doesn't need us, Netflix has David Fincher to bring in to direct. They're at the highest peak of Hollywood...I think that going and pitching and doing an original series for Netflix is about as realistic as pitching and creating a new series for NBC. It's like, 'Okay, it's you or Tina Fey.' It's like, 'Well, that just ain't gonna happen.' (Independent production company executive)

Another production executive commented,

It's very difficult, because you are competing with Steven Soderbergh...All these kinds of major Hollywood players. So you need to have something that they're gonna go, 'Yeah, okay, l'd like to work with that person.' So just the original content...is very difficult. (Independent production company executive)

Another jokingly commented on the weakness of his position, "My pitch to Netflix is, 'I can produce a video for you that will give you a $0.1 \%$ return on your money'" (Independent production company executive). In addition then to the challenge of competing with the world's preeminent cultural producer for original content deals, most digital-first Canadian series, made by independent creators and firms alike, have been short-form in nature. According to creators, as mentioned previously, this does not appear to be the type of content the high-end digital streaming firms are interested in:

[O]urs are very short pieces, 10 minutes... is the longest [episode]...[T]hey're not interested in running that, Netflix, and Hulu, and Amazon. They want TV series, if 
they do want it at all. That's the other thing. Amazon's a little bit more flexible, but Netflix, they're curating, they don't want just everything under the sun, they want stuff that they feel is worthy.

The ability to package high-end talent together on projects was also reported as a weakness for Canadian firms by an executive at an established production company:

[W]e are falling behind in our ability to package. Packaging is huge...And we wanna get better at it... Packaging in the US is run by essentially the agencies...The director and talents and the writer have all come from the same agency, and so it's them going out doing the deals ... Not in every case, but you see that a lot. So that is a barrier to entry... having access to the resources to package... (Independent production company executive)

With the limited resources at their disposal, most creators felt that getting an original

Netflix deal without a pre-existing audience, or pre-existing (successful) intellectual property was unlikely. This was especially the case for independent creators who were not affiliated with firms:

I don't find it to be very realistic [securing a Netflix deal], 'cause there's so much involved with a successful show that I think there are several web series out there that, yeah, you know, they're good, but it's the combination you need to make a successful show marketable to the audience base of... Netflix. There's so many pieces that need to come together. And I'm not sure that the indie environment necessarily has those. Especially with web series, you often have one or two people doing a plethora of roles that if you had a whole team of people, which people can't afford to spare, you would really have a more developed concept and idea. You can certainly try to pitch to Netflix, but when you see the sea of content that is out there, to get Netflix on board, thinking it will find an audience, if you can't even find an audience online? (Independent creator)

Thus far, Canadian Netflix originals have been series created with established intellectual property (Trailer Park Boys; Degrassi; Lost \& Found Music Studios), original 
series produced in partnership with Canadian broadcasters (Between; Travelers;

Frontier) or both (Anne with an E; Alias Grace).

\section{Discussion and Analysis}

Although their motives for challenging and re-writing the status quo are grand, Canadian web series creators face considerable challenges from both outside, as the previous chapter revealed, and inside the Canadian system. The extra-national institutions and forces largely dictate the shape of the playing field in which they operate, carving out and delimiting its outer boundaries, while the Canadian ones present impediments to their capacity to fully engage in, and exploit, the opportunities within that playing field. These two external and internal elements are not entirely separate, often overlap, and constantly interact. They coalesce into a unique set of factors that are different from those experienced by web creators based in other jurisdictions. By viewing web series creators as entrepreneurial agents who fundamentally aim at change, and examining their decisions against the backdrop of the structural forces they interact with, the process of structuration again becomes more visible. Indeed, although many Canadian web creators aim to use the web to escape or tear down the rigidities associated with the domestic broadcasting system, they are still, on many levels, subject to its influence even when engaging in this supposedly borderless space. Indeed, the importance of geography is reinforced as 
the various domestic elements examined here have demonstrated impacts on Canadian web creators' entrepreneurial journeys.

At the domestic level, Canadian independent creators and firms often do not have the skillsets required to fully exploit web-based opportunities, and do not have the resources to hire skilled talent. Even when they do, there appears to be a dearth of talent with the required skillset to maximize chances for success. Canadian broadcasters also appear to be less than optimal partners in the exploration and exploitation of the online space, due to either a lack of commitment, or a lack of understanding of the web, or both. The lack of commitment and eagerness to change is also not uniform, and several broadcasting executives working at the majors expressed a desire for transformation, but ultimately felt restricted by existing policies, hierarchies and systems outside of their direct control. This push and pull between micro-level, human agency and the more macro-level forces illustrates the complex process of change.

The general absence of clear, digital strategies on the part of Canadian broadcasters, has, in many cases, resulted in web series being 'buried' in the digital ether, with little hope of being discovered by audiences on platforms that have been inconsistently supported or abandoned. The current funding programs that do exist are also built around models designed for television, and do not fully take into account the unique elements of the web, or its realities. The funding system reinforces and 
perpetuates the issues as well, given that the most significant financial allotments are still earmarked for Canadian broadcasters. The Canadian performers' union also appears to have positioned Canada's more established talent outside of the scope of many web creators and their projects. The worthy and important goal to protect actors may have the unintended effect of hindering the advancement of the nascent web screen industry along a number of fronts, as well as limiting opportunities for unionized actors to be seen in meaningful roles and to practice their crafts. Finally, because of limited resources, limited relationships, and a lack of history of directly competing with Hollywood for the same 'shelf space', for most Canadian creators, capturing the attention of high-end US-based streaming firms is a challenge. The high-end digital streaming firms are interesting from the standpoint that they are firms with primarily NoCal origins, but value SoCal Hollywood crafts and have production strategies informed by NoCal sensibilities. While Canadian independent web series also demonstrate commitment to SoCal time-honoured Hollywood crafts, they are generally not developed enough, for all of the reasons explored, to appeal to this calibre of buyer in their original forms. Web series, in general, are thus stuck between the proverbial 'rock and a hard place', not fitting the type of content ideally suited for YouTube, and not advanced enough for high-end digital streamers. The physical distance from Hollywood also places Canadian creators at a disadvantage in the sense 
that opportunities for face-to-face networking with executives from the streamers, and other high-end partners, are also greatly reduced.

The potential for brain drain appears to be emerging on two-fronts: the first in a digital sense, wherein Canadian creators seeking online distributors with considerable subscribers in the millions look to partner with US-based online YouTube channels since no Canadian broadcasters with subscribers at these levels currently exist; the second in a physical sense, wherein some Canadian creators have admitted to shifting or showing interest in shifting production to the United States where the performer union's new media agreement is more flexible.

The next and final chapter begins by bringing the focus back to agency, and explores the kind of change that web creators have been able to achieve through their entrepreneuring despite the many systemic and structural forces emanating from both within and outside of Canada that challenge them. The next chapter will also reiterate the merits of the hybrid political economy-entrepreneuring approach, and will synthesize the findings of the work developed here to provide meaningful program and policy recommendations. 


\section{Chapter 8 - Conclusion: Encouraging Canadian Screen Industry Change by Empowering Web Creators}

The last three chapters of this dissertation have detailed the challenges faced by Canadian web creators in the pursuit of their goals. Along the path of their entrepreneurial journeys, they collide with an intricate and intersecting nexus of obstacles that emanate from various sources including their own identities and positioning in the wider socio-economic hierarchy, US-based new media virtual infrastructure, wider, global cultural and social trends, as well as the Canadian screen system and its various institutions and unique features. Despite all of this, Canadian web creators, through their entrepreneuring, have, in many cases, been able to ameliorate their own or others' positions in the system they reject, or as is often the case, the one that has rejected them as they work to build a new order that reflects their values. The first part of this chapter will be devoted to examining changes creators have been able to create through their agency and entrepreneuring. A discussion of the major findings of the dissertation follows. The final part of the chapter will then provide recommendations for policies and programs that aim at encouraging, amplifying and extending the change creators have been able to bring about, and that which they seek. 


\section{Making Change: Beyond Economics}

Although web creators often had trouble reaching the goal of achieving economic success through their series, they were able to create important change for themselves, and sometimes for others through their acts of entrepreneuring. These outcomes will now be discussed below.

\section{i. Learning \& Skills Development}

Many creators, including established production company executives, emphasized that through the production of web series and by being involved in many or all aspects of production (something that would not normally be the case in traditional television), they were able to gain a more complete understanding of the entire production chain, and strengthen their grasp of the production process which they can then apply to subsequent projects:

On the personal side for the creatives who work on it, it builds a tremendous amount of scale. It's an incredible learning experience about the production as a whole because as soon as you get to the web, all the lines between these traditional silos blur. You learn much more about what everyone else does on a production so you become a much more well-rounded artist. (Jill Golick, Independent creator)

Another creator agreed, noting that the freedom "to just do anything and then see it on screen" provides a type of learning experience that no other medium, because of how it has been institutionalized, can provide (Independent creator). The creator recalled that his web series was permitted "to get weird" and then return "to a 
more composed thing", as he and his production partner developed a deeper understanding of the series, the audience, and production in general, noting he doesn't believe he and his partner "could have learned those skills" otherwise (Independent creator).

The wide scale of the creative skills developed through online series also prepares web series creators for potential future careers as showrunners. The showrunner is a top creative overseeing role that is commonplace in Hollywoodproduced television, and is thought to be a key contributor to (American) television's current "golden age" (Berkowitz, 2017). The showrunner is "in charge of preproduction, production, and post-production. In other words, everything" (Jean et al., 2012 as quoted in Berkowitz, 2017). The model of production that embraces the showrunner, however, has yet to be embraced in the Canadian industry (Berkowitz, 2017; Blinning, 2010), and there appears to be a dearth of showrunner talent due to brain drain to the United States (Berkowitz, 2017). If allowed and encouraged to develop, Canadian web series creators are excellent candidates to fill in this important gap.

Importantly, as well, through their work, creators also learned about the skills that they were lacking (as discussed in the previous chapter), and often felt more prepared for subsequent projects because of their newfound awareness. As one digital production company executive noted, 
[The series] showed us that we are not prepared to be in linear online video production. And so some people might see that as not being a success because [the series], we're not putting further resources into it, and we're not pitching it as a future viable business plan. But everything that I've just told you for the past 90 minutes was learned through the production of [the series]. (Independent digital production company executive)

And though the series itself, for this creator, was not economically successful, his firm

has been able to apply the skills learned throughout the process to future projects that have been profitable in an area outside of, but related to, scripted web series.

\section{ii. Career Acceleration}

Closely connected to skills development was the way creators were able to harness their experiences to propel themselves forward in the screen industry. Web series, for early stage creators, often served as career accelerators. As one creator reflected:

It's gotten me work. I've had people hire me for other projects 'cause they saw Pete Winning. It developed my acting career. I wasn't an actor before Pete Winning and now I am one. I've had the lead role in four feature films since Pete Winning and yet, three years ago, I wasn't acting at all... But a lot the actors have gotten work because of Pete Winning, or we've had many of our cast members nominated for acting awards and stuff at festivals and whatnot...This was one of the greatest things in my career that I feel I've accomplished from a personal level... (Mike Donis, Independent creator)

Another commented,

To date, between season one and season two, we've been nominated for, or won, 77 major awards...Insanely successful. It was a moment that I look back on and think 
like, 'Wow, three years of just awards piling in...that will never be repeated'. (Independent production company executive)

Many creators also noted how web series have helped them move from volunteer to paid work:

I've known people that have volunteered to be doing sound on sets and now they work professionally as sound operators. Our sound operator for [our show], is now a professional sound operator. He's been working all over the world as a sound technician. (Independent creator)

One creator of a popular web series noted how his show has given him the capacity to develop the kind of track record needed to more fully participate in the Canadian screen system:

We have this [other] show, and people are interested in it and people that have seen our stuff are willing to accept pitches, and they probably would have never done that, outside of that. So it's been very critical. In terms of the general profile of myself as an artist and also this company that I have created and I'm trying to build, it's been quite important to be able to have the awards and nominations, and reviews and the views, and all the bullet points that are needed to make the necessary introductions for things... It's been above all a major, major step for professional development. (Independent startup production company executive)

For early stage creators, therefore, developing a record of awards and other successes opens the door to other competitions and resources, and allows them to accumulate the right kind of "points" needed to succeed in pitching and funding applications. This significantly ameliorates their position within the wider system, and increases their chances of success. 
Several creators stressed that one of the most important outcomes of their web series experience has been the development of their brand. Jeremy Larter, creator of Prince Edward Island web series, "Just Passing Through", commented,

I think it's a good way to build a career...or to get your name out there... They wrote a Toronto Star article and they featured it in their article. That was just a minor little success online. So I think if you're able to get your name out there and get your face out there and build... I hate the term 'brand', but that's what it is...Or basically, if you find your fan base who likes your stuff, then you can maybe springboard that into something else... (Jeremy Larter, Independent creator)

Since the interview, Larter and his team have been able to leverage the brand they created, citing "the same stunned arseholes who brought you the comedy series 'Just Passing Through'", in a successful crowdfunding campaign that raised over $\$ 55000$ for another Prince Edward Island-centric project, only this time, a feature film (Pogey Beach, 2016).

Many creators have also used their web series as pitching tools. Creator Hannah Cheeseman, who was interviewed for this project, currently has a television show in development based on her web series, "Whatever Linda", with The Mark Gordon Company, an American-based studio headed by Mark Gordon (Andreeva, 2016), producer of hit American series "Criminal Minds", "Grey's Anatomy", "Private Practice", and many others. 
Other creators stressed that the relationships they have been able to develop

because of their work have opened up new opportunities for them. As one executive at a digital production firm recalled,

...The relationships that were formed during [the series] have generated hundreds of thousands of dollars of future production work...[The series] lost substantial amounts of money...but the spin-off benefits have been enormous. (Independent production company executive)

Another young creator recalled how, after seeing his web series online, an established producer reached out to him:

He tweeted about the show, 'Oh I love this show... Check it out.' And then he messaged me...Literally a cold message from him, 'Are you interested in developing [your series] for a network?' And we wrote...scripts together... And we just have been pitching it around to people. (Independent creator)

Web series therefore, have allowed some creators to break through and to utilize the attention, relationships, brands, audiences, rewards and skills to move their careers to the next level.

iii. Social Good

Several creators with larger social missions felt that their series were able to make an impact in their communities of interest. Creator Jason Leaver recalls receiving emotional messages from fans from around the world regarding their struggles with being gay, and how his series played a profound role in their journeys:

We've had audience members tell us, literally... One of them... I remember saying, 'It was a year ago, today, had I not discovered Out With Dad, I probably would 
have died, because I was suicidal, but I found your show, and I decided to keep watching', ...And that's not a unique message; we have lots of messages. The cast, they get messages privately too, on their Facebook pages, and telling stories like, 'No, no, no, you saved me', or, 'I'm proud of who I am now', like, 'I thought I was alone...'. Talking about a global audience, we got it pretty good in Toronto if you're coming out, and the show certainly reflects that, what it's like in Toronto, but then you got people around the world where, it's like, 'Oh, there are other people like me?' ...We've saved lives, that's success. (Jason Leaver, Independent creator)

Creator Yusuf Zine described how his series, about the plight of a visible minority actor

in Toronto (based on his own experience), is now being used as an educational tool.

For him, this outcome was significant:

[B]eing a minority, it was very liberating... because one unexpected outcome that came out of this was university professors got a hold of 'Fame and Fidelity' and they wanted to show it in their classes and I still get messages from professors saying, 'Will you do a talk about this?'...So in terms of that educational aspect it's been, I think a huge success, because it has been used as a learning tool to show how creative art can be used to resist dominant narratives...

By being able to authentically reflect his own struggles in his web series, Yusuf believes his project has brought attention to issues of discrimination in the Canadian screen industry.

One creator felt that the collective success of web series creators in achieving awards in the international community has helped to elevate the reputation of the Canadian screen industry at large:

I think that there is great value in what l've learned... not just for me, but for the industry as a whole. I think I brought a lot of critical acclaim to the industry from the show...It is the first time I think that in the history of these industries Canada has been able to say that they have been the world leader in any medium... (Independent creator, Jill Golick) 
Many creators felt that Canadians were leaders in the production of independent web series, and felt inspired by what they viewed as the overall positive momentum produced by the Canadian web series community, despite often not achieving the financial success they originally envisioned.

Discussion

This section has demonstrated that through their entrepreneuring, despite the challenges, and in the absence of many of the traditional television industry supports, Canadian web series creators have been able to enact change for themselves and for others. They have developed new and tangible production skills where opportunities to do so were few. They have developed better and well-rounded understandings of the production process, and gained an intimate knowledge of, and relationships with, their audiences and key demographics. They have also developed an appreciation of the unique resources required to maximize chances of success in the digital-first space, as well as their own limitations in the area. Through their work, they have been able to shine a light on issues meaningful to them, told the kinds of stories that have been systemically excluded and have reached communities that have been overlooked. Canadian web creators have secured gigs, licensed and sold their series internationally and here at home, won prestigious domestic and international awards, grown viewership in the millions, succeeded in advancing in the Canadian storytelling system 
by winning competitive grants or funding, run successful crowdfunding campaigns based on the brands they have painstakingly grown, and have used their web series as proofs of concept to successfully pitch television studios both here and in the US.

These successes are a testament to their perseverance, creativity and entrepreneurial spirit, and have occurred in spite of the many challenges that act as barriers along their paths.

\section{Reflection and Overview}

This research project sought to examine the emerging Canadian digital-first industry, and its associated cultural practices and texts, and the actors, politics, policies, and social, economic and institutional forces that have shaped and are shaping its development, including its emergent patterns of inclusion and equity. By developing and employing a novel expanded critical political economy approach that combines the critical political economy of communication with critical entrepreneurship studies, and views web series as acts of entrepreneuring that aim at emancipation (Rindova et al., 2009), the work developed here has been able to mediate effectively between structure and agency to reveal how Canadian web series creators are interpreting, internalizing and resisting larger institutional and structural dynamics and discourses in their cultural practices and texts. 
The hybrid, critical political economy-entrepreneuring as emancipation approach has made visible the motives and goals of Canadian web creators while situating them within their wider social, political and economic context. More specifically, the entrepreneuring as emancipation framework's focus on processes rather than entities, when combined with the critical political economy approach to structural analysis, has permitted a deeper grasp of the genesis of web creators' desires to break free from, and disrupt the existing screen system, and has allowed for a complex and nuanced understanding of the various systemic and institutional challenges faced by web creators in the pursuit of their goals to emerge. The study's research questions around web creators' motives for engaging in the web series space, and the perceived opportunities which lead them to engage, have thus been answered in a robust way, and in a way that, as will soon be shown, can be used to meaningfully inform policy in this area. The resulting picture is a thus complex one in which it has become clear that Canadian web creators have been both inspired to act in a rejection of the larger systems in which they are embedded, but their successes, and indeed the potential of their agency, have also been tempered by the many forces and institutions that emanate from Canada and abroad. The dissertation's sustained focus on balancing agency and structure, through the examination of agency always within its wider political-economic context, has allowed for a dynamic understanding of actual (versus perceived) opportunities to also emerge: 
On the route toward the pursuit of their goals, Canadian web creators have been shown to be greatly affected by larger shifts in audience expectations around the value of online content, the complex nature of the Participatory Culture Paradox, which, while enabling their work, erodes its worth, the apparent dearth of successful economic models for Canadian independently produced web series that value SoCal time-honoured crafts, the volatility and opacity of global platforms that dictate the terms of their participation, the crystallization and centralization of these platforms and the increasingly asymmetrical power relationship between the platforms and the creators who populate them with their work. The study has also revealed how the tension between art and commerce has material impacts on the livelihoods of creators. The recent treatment of LGBTQ content by Google's YouTube demonstrates the precarious status of the creator on these platforms.

Furthermore, on the Canadian front, the study has shown how creators lacked certain key skills and resources, and were generally apprehensive about the future of the Canadian media system, and the possibility of furnishing successful screen careers in their home country in the future. They were challenged by Canada's broadcasting leadership, its lack of apparent cohesive and consistent strategies and commitment to those strategies, and found Canadian broadcasters to often be less than optimal partners. Creators were also restricted by existing industry norms that they believe have not been well adapted to the realities of the online space, including the new 
media agreement for union actors, as well as the structure of funding programs which are still designed largely around traditional televisual practices. Creators, at all levels, also found it difficult to attract the attention of digital streamers both here and abroad.

The hybrid critical political economy-entrepreneuring approach has thus provided a novel method of unravelling the complex and multilayered process of structuration, which traces the interaction, and mutual constitution of individual agency and structure. As Mosco (2009) contends, structure is the platform from which human actions emanate. It is clear, therefore, how Canadian web creators have reacted to the various structures in their worlds through their entrepreneuring in an effort to create a new order for themselves and for others. Dissatisfaction with certain structures in their worlds thus motivates them to act, and they use their individual agency, while navigating these structures and forces, to create a different kind of system, as best they can. Each individual act of entrepreneuring coalesces together to form the Canadian web series movement and joins what is likely a global movement of people who engage in online creation in an effort to challenge existing screen industry norms in their own jurisdictions, and to establish new norms and practices that reflect their values.

This is precisely what Christian (2012a) found in his examination of the US-based web series production ecosystem. There, he discovered disenfranchised creators who engaged in entrepreneuring to challenge the stratified Hollywood system that was 
unwelcoming to their stories and their labour (Christian, 2012a). While the sources of dissatisfaction are specific to each jurisdiction, and are of immense import when weighing public policy options-the global web series movement demonstrates the universality of struggles for power over communicative resources. Furthermore, the work developed here has also picked up where Christian's (2012a) left off. Christian (2012a) focused on what he views as the web series medium's peak years between 2006 and 2010, a period in which there was ample optimism that an independent market for creators could exist. The work here has thus focused on, to borrow Christian's (2012a) terminology, the medium's post-peak years, 2010-2015. As Christian (2012a) anticipated, there has been increasing institutionalization and crystallization in the web space, as evidenced by the wide array of challenges experienced by the web creators examined in this work.

As for the promise of the participatory culture era (Benkler, 2006; Burgess and Green, 2009; Jenkins, 2006; Shirky, 2008; Tapscott and Williams, 2007)—while certainly individuals have more access to media than ever before, as others have pointed out, this participation is highly determined and pre-structured by corporate platform owners that have complete power to dictate the terms as they see fit (Butler, 2016; Kelty, 2013; van Dijck, 2013; Wasko and Erickson). For users who are not in the business of professional content creation (or trying to be), when a platform changes its terms of trade or one of its algorithms, the results might be inconvenient, perhaps even 
annoying. The stakes are much higher, however, when one is a professional creator, and as this dissertation has shown, the consequences of such changes can have real and material impacts on creators' businesses, livelihoods and most fundamentally, on their capacity to even have their work seen. A distinction must be made therefore, when making claims regarding the scope of possibilities that participatory platforms offer for regular users versus those who aim to, through their participation, develop professional careers and professional, alternative media products. There is ample room for future research that examines this important topic.

Importantly as well, it is crucial to note the uneven effects that democratized access has for both regular and professionally oriented users. While the participation enabled by Web 2.0 platforms has generally been a positive force for normal users, and has enabled the path for web creators to share their work, it simultaneously reduces the value of their work, as audiences see fewer reasons to pay for content in a world of infinite abundance and substitutability.

Related to this and in direct response to its research question surrounding the genre and orientation of content that is produced for the online space, the dissertation has also demonstrated the importance of examining different online content forms separately. While the web has certainly opened up new avenues for business in the area of storytelling, content that adopts the lean and rapid NoCal logic that is inherent in more web-organic forms such as vlogs, has a greater chance of finding economic 
success and sustainability on platforms like YouTube than scripted web series which adopt the SoCal approach to developing time-honoured Hollywood crafts such as acting, writing, directing, editing, composing and cinematography. Content is not treated equally by Web 2.0 platforms, and the way participation and access are structured at the level of code has significant impacts on whether and how creators can get their work seen, and make a living.

Through its mixed-methods approach, the study has also been able to answer its research questions surrounding diversity and the various barriers to access and participation that exist in this space. The work here has found that in the Canadian sphere, self-funded web series, in which there is no external, formal gatekeeping apparatus, appear to provide important inroads for the participation of women and visible minorities in roles in which they are most underrepresented in traditional film and television; for women, this is particularly true in the category of director, and for visible minorities this is true in the categories of both director and writer. Although women and visible minorities continue to be underrepresented across all categories examined in self-funded series, especially when compared to their representation in the general workforce, and, for visible minorities, their regional representation, the situation is much worse in more professionalized, funded web series, where the outcomes closely resemble those in the traditional television and film space. 
The dissertation has thus revealed that a great danger then appears to exist in extending the usual model of funding found in traditional television to the web series space without modification. The replication of the kind of system that is unwelcoming to women, visible minorities and other new voices coming from outside of established networks is a potential outcome of such an extension.

Numerically speaking then, while Cunningham and Craig's (2016) observation that there is increased diversity on social entertainment platforms is likely true in the aggregate, certain content streams, due to their fundamental features, tend to repeat patterns present in the traditional cultural forms upon which they are based. Scripted, narrative web content is more resource and skills intensive than vlogging. Its intensified model of production, like the general creative industries model of production (Eikhof and Warhurst, 2013), confers advantages on some and disadvantages on others. As explained in detail in Chapter 5, the importance of socioeconomic filters (gender, race, age, social class) in the intensified production model of web series diminishes some of the effects of the freedom provided by the nearly unfettered access to online distribution provided by Web 2.0 platforms.

\section{Harnessing the Potential: Policy and Program Recommendations}

A key area of priority for the study was the identification of policy

recommendations based on the study's findings. The unique, expanded CPE approach 
developed in this dissertation has several benefits for the development of policy in this area. To begin, expanding the scope of entrepreneurial activity to include web-first creation connects two policy domains—cultural industries and entrepreneurship/innovation respectfully, which have historically been siloed in Canada. This has erected artificial barriers between art and commerce which may have impeded the development of potentially fruitful interactions that could have opened up possibilities for alternative, commercial media systems that work better (more inclusively, more openly, more transparently).

Secondly, the study's attention to the contextual dimensions undergirding entrepreneuring in this sector, combined with a focus on the processes of breaking free and breaking up, as shown, reveal significant details about the "who" and the "why" of web creation in Canada. By giving context the attention it warrants through the expanded CPE approach, the dynamics of the sector become clear, and policy can be adapted so that it better responds to "who" becomes involved, and "why".

Thirdly, as Rindova et al. (2009) point out, those doing the entrepreneuring "may have only a limited understanding of the solidity of the structures they seek to dislodge" (p. 479). These shortcomings need to be considered through not solely an entrepreneurial policy lens, but also through wider social and cultural mechanisms so that these individuals may be empowered to succeed. Policy that is tuned into contextual dimensions can better attend to the needs of entrepreneurial actors and aim 
at removing barriers and constraints extending from wider socio-economic factors and forces.

As many scholars have painstakingly shown, western creative industries policy has tended to depict work in this sector as highly desirable, flexible, socially inclusive and meritocratic, and a panacea of economic growth and good jobs (Eikhof and Warhurst, 2013; McRobbie, 2004; Oakley, 2006, 2013; Ross, 2006). These depictions belie the harsh realities of work in this space. As Oakley (2006) rightfully points out, there is a danger when creating policy around creative entrepreneurship to overstate its capacity to transform work and restore social cohesion. It is imperative, therefore, to keep in mind the wider range of socio-economic factors which act as mediators to participation.

Canadian web creators' entrepreneurial ventures were often direct responses to the lack of opportunity to find meaningful work in the traditional television sector. Many of the creators interviewed were reluctant entrepreneurs. If meaningful work in the scripted television industry in a non-entrepreneurial capacity were available to them, they would have preferred that arrangement. Only a small group of those interviewed could be said to genuinely be seeking to build businesses in this new space. This means that screen sector policy must acknowledge and tend to these two, differing orientations. It must empower and embolden would-be entrepreneurs 
regardless of background, but also focus on the creation of good jobs and ensure equitable access to them.

Because of the wide breadth of discoveries made through this dissertation, the recommendations offered here will primarily be aimed at setting larger priorities for the Canadian digital series space, rather than at providing comprehensive and granular program or policy guidelines. The recommendations provided consider the digital series space not in isolation, but in the context of the wider Canadian screen industry and its operations, particularly given the dissertation's findings around the motives behind Canadian web entrepreneuring. Before the recommendations can be presented, however, more policy context must be provided because as web creators have been working to elevate themselves through their entrepreneurial efforts, wider domestic policy changes are having significant impacts on the stability and shape of the established Canadian system that so many of them are looking to break into. These wider issues will now be explored, and the blueprint for policy and program recommendations aimed at harnessing the potential of web series, and applying the lessons learned through this dissertation, will follow.

To begin, as viewing of linear television and cable revenues continues to decline in Canada, the majors' contributions to the country's central production fund, the CMF, will decline as well. For the 2016/2017 year, the program budget for the CMF reflected 
a decrease from previous years, in anticipation of reduced contributions from the majors (Cummins, 2016).

The CRTC recently reduced the amount Canadian broadcasters must spend on 'Programs of National Interest' (PNI), or Canadian dramas, documentaries and award shows (CRTC 2017-148). The change, according to certain industry groups, is expected to result in a 200 million dollar decrease in funding available to Canadian production over a five-year timespan (Reid, 2017). In August 2017, after pressure from creative groups, the Governor in Council issued an order for the CRTC to review this decision (Jackson, 2017). It is unclear what the result of this directive will be. In addition to this, the CRTC also recently loosened requirements around the number of key production roles that need to be occupied by Canadians in order for productions to be certified as Canadian and therefore be eligible to receive funding from CRTC-certified independent production funds (CRTC 2016-343).

The change has sparked concern across Canada's creator community that there will be even fewer opportunities available to Canadians in an already strained and declining system in which there is a dearth of original scripted production (Reid, 2016). Several of the funds affected are open not only to traditional television projects, but web projects as well. In its major policy review of the state of the television sector in Canada initiated in 2013 through its "Let's Talk TV" hearings, the CRTC opted to maintain the longstanding exemption order on new media. This means that no online 
services (both domestic and foreign), are required to contribute to the funding of Canadian content, or to carry or commission any Canadian content at all.

In these hearings, the Commission actually expanded the exemption order by creating a new "hybrid" category of service (CRTC 2015-86) that exempts previously regulated video-on-demand platforms based in traditional delivery systems (cable, satellite, IPTV) from all Canadian content requirements, provided that broadcasters make the same pay service available online to all Canadians on an OTT video-ondemand platform. Canada's sole mainstream OTT service, Crave TV, falls under this category, and thus has no Canadian content requirements. These decisions were made, according to the Commission, to provide the traditional television system with the "flexibility necessary to operate in an increasingly multi-platform environment" (CRTC 2016-343).

The problem, however, is that while, as was made clear in Chapter 4, it is certain that negative externalities have emerged from Canadian broadcasting policy, the key reason a Canadian market exists at all, is the policy system. While the online world is in many senses borderless, place is still important for Canada's creators, and they need opportunities in their own country to participate in creative work that provides both an income, and the chance to meaningfully develop and grow their talent and skills. While the goal of developing a more competitive, innovative and entrepreneurial screen system is valid and necessary, the policies implemented thus far fail to acknowledge 
the harsh realities of the economics of the digital ecosystem, as detailed throughout this dissertation.

Since April 2016, however, the Trudeau government has been leading a consultation on "Canadian Content in a Digital World" (Canada, 2016). The goal of the consultation was to be a policy framework "on how to strengthen the creation, discovery and export of Canadian content in a digital world" (Canada, 2016. p.2).

Because the new policy strategy, "Creative Canada" was only recently announced, few details are available at present. In the section that follows, the details that are known, however, will be discussed alongside the study's recommendation. This will begin to provide a sense of how well the new strategy addresses the real-world needs of Canadian creators.

i. New Sources of Funding for Canadian Content

To start, it is clear that despite new economic opportunities enabled by the web, making a living as a Canadian scripted web series creator is a challenging proposition. Because of the realities of the economics of the digital space, the need for public funding of Canadian content remains, despite CRTC Chairman Jean Pierre Blais' assertion that the web has enabled "unprecedented opportunity", and "extraordinary possibilities" and opened "doors to niche markets unimaginable even a decade ago" 
(Blais, 2013). This means that updating sources of funding to support Canadian storytelling is vital.

Sources of funding for the CMF should thus be expanded from revenues from the majors' cable, satellite and IPTV services. The internet has become an important, and increasingly central access point for audiovisual cultural content. The funding infrastructure for the CMF should therefore be updated to reflect this. In its new cultural policy, the Canadian government has announced that it will increase its contribution to the CMF to restore the decrease that has occurred on account of declining distribution revenues of the majors (Canadian Heritage, 2017). While this might be an appropriate stopgap measure, it is unclear what the government's strategy will be should the majors' contributions decrease significantly.

As suggested by many (see for example, Grant, 2008), including a recent report from the Heritage Standing Committee (Standing Committee on Heritage, 2017), one potential new funding stream for Canadian cultural content could be revenues generated through the majors' internet services. Under current legislation, which separates broadcasting and telecommunications, and places the former under the purview of Canadian Heritage, and the latter under Innovation, Science and Economic Development Canada, this might be a difficult undertaking. The Broadcasting Act (1991) contains both a cultural and economic mandate, while the Telecommunications Act (1993) contains only an economic one (Skinner, 2008). Complicating matters is the 
fact that in 2012, the Supreme Court of Canada ruled that internet service providers are not broadcasters because they have no "control over programming" and thus should not have to contribute to the broadcasting system (2012 SCC 4).

Significant change to funding sources might, therefore, require deeper and more complex legislative change, including a potential merger of both legislations, as has been suggested even by the CRTC (2008). The political will to expand CMF funding to include revenues from the majors' internet services does not appear to exist, and recently the Trudeau government publicly announced it would not support such a strategy (Blatchford, 2017). The newly announced cultural policy strategy does set a course for reviewing the Broadcasting and Telecommunications Acts (Canadian Heritage, 2017). The timeline for these reviews is unclear.

The new strategy also takes a clear stance on one potential funding source that has received considerable coverage in the press: the possible redistribution of revenues generated by domestic and foreign streaming services in the Canadian market. This proposition received ample public scrutiny in recent years, and became a political issue (the "Netflix Tax") in Prime Minister, Stephen Harper's, campaign for the 2015 Canadian federal election (Zboralska and Davis, 2017). The government's new strategy clearly states that it will not impose any tax (not even sales tax) on online services as such taxes would serve to "increase the cost of these services to Canadians" (Canadian Heritage, 2017, p.26). 
Instead, to increase demand for Canadian content, the government, in its new strategy says that it will be seeking "commitments from, and pursue agreements with, global Internet companies that provide services to Canadians" and that the "expectation is that these companies will be partners in, and contribute to our objectives for, Creative Canada, helping grow our creative industries with investments in production and distribution" (Canadian Heritage, 2017, p.26). The first of these deals is a commitment from Netflix to spend $\$ 500$ million over five years, and to establish "Netflix Canada - a permanent film and television production presence here in Canada" (Joly, 2017). Since the initial announcement, the government has clarified that this spending will not be on American-based productions filming in Canada (Parker, 2017). The reactions to this aspect of the announcement have been polarized. Some creator groups have embraced the deal, noting that the global reach of Netflix's service can have profound impacts on the discoverability of Canadian content in the context of the digital world (Parker, 2017; Reid, n.d.). Other stakeholders are greatly concerned about what this deal might mean for Canada's long-term cultural sovereignty. Karl Peladeau, president and CEO of Quebec communications giant Quebecor remarked,

[T] he government's approach will lead, logically and inevitably, to domination of Canada's broadcasting ecosystem by U.S. giants. The public broadcaster will be the only remaining domestic player, as the private broadcasters' manoeuvring room will have been wiped out. Ultimately, local producers, artists, cultural workers and audiences will be the losers. (Peladeau, as quoted in Reid, n.d.). 
In the context of the findings of this dissertation regarding the increasing consolidation of attention in the online ecosystem to a few platforms and services, Peladeau's concerns appear valid. On the other hand, with domestic broadcasters proving to be less than optimal partners in the production and distribution of scripted stories (on television and over the web), the creation of more meaningful opportunities is a welcome element. Many important questions remain regarding the nature of content that Netflix will be looking to pursue in Canada. It is also unclear whether the government will be able to continue to secure Netflix-like deals with other streamers, or whether such a deal will be a one-off. More deals of this kind have the capacity to substantially increase opportunities for Canadian creators in a way that will get their content seen.

As has been shown, however, many Canadian web creators are at the early stages of their careers. It is unclear whether the new Netflix deal will offer opportunities for these early stage artists to get involved. Thus far, Canadian Netflix originals have been series created with established intellectual property, original series produced in partnership with Canadian broadcasters, or both. Netflix has therefore, primarily entered into partnerships with established firms, rather than emerging, independent creators and companies in Canada. In the absence of additional strategies to encourage new voices into the Canadian screen system, a very real danger of the 
Netflix deal (and others like it), is thus the perpetuation and emboldening of networks of exclusion.

\section{ii. Designing Funding Programs that Encourage New Voices}

A clear finding from this dissertation is that current project funding models, and the way funding is allocated, need to be adapted to encourage the entry and participation of new voices, including women and visible minorities. This is especially the case now, in light of the new Netflix deal, given the company's historical pattern of partnership, as discussed above. As Chapter 5 has demonstrated, the participation of women and visible minorities is reduced in the key creative roles of writer and director in series receiving IPF funding.

In what follows next, an analysis of existing funding programs will reveal that an even more entry-level program, designed with the aim of encouraging a wider array of new voices, should be considered.

Both regional and national funding programs exist for Canadian web creators. At the national level, Canadian independent web creators can apply to several funds including the CMF Web Series Pilot Program (public fund), the IPF (private fund), and the Telefilm Micro-Budget Program (administered by the public Telefilm, funded in part by Telefilm, and private donations). While each province has its own funds as well, the example of Ontario will be used below to illustrate the issues in a concise manner. 
Thus, in Ontario, web creators based here can apply for the Ontario Media

Development Corporation (OMDC) Interactive Digital Media Fund (a public regional fund).

Only one of the programs mentioned, the Telefilm Micro-Budget Program, is geared specifically at creators at "emerging" stages of their careers. The remaining programs are either open to creators at all career stages (IPF) or are geared at more established creators (CMF and OMDC), and in the case of the CMF specifically, at web series past their first seasons that can demonstrate clear market traction (CMF, 2017b). For each of these programs (other than the Telefilm Micro-Budget Program), the track record of the creative and production team are key considerations in assessments for funding. The CMF and IPF also expect recoupment from projects (CMF, 2017b; IPF, 2017).

While the Telefilm Micro-Budget Program (open to both feature film and narrative web series projects) is specifically aimed at "emerging" creators, certain particularities of the program might, inadvertently, act as barriers to the entry of new talent. For one, the "key members" (writers, directors, producers), of all projects submitted for funding consideration must fall under the program's definition of "emerging talent" in that "they must already have produced, directed and/or written at least one short film (i.e. 30 minutes or less) but must not have produced, directed or written a feature length film (i.e. 75 minutes or more)" (Telefilm, 2016a), and if applying 
for web series funding, they must never have "produced, directed or written a web series" (Telefilm, 2016b). These guidelines would appear to exclude those who have engaged in these roles on web series in even student-based web projects, and in selffunded web projects that have never received funding. This is strange given that the guidelines permit and indeed require those applying for funding to have worked on at least one short film of 30 minutes or less. It is unclear why an individual who has worked on a web series of the same total length should be restricted from applying.

Secondly, the program is open only to recent graduates of select, "designated" training institutions or "active members" of film cooperatives who must receive a nomination to compete (Telefilm, 2016a). These partners are only permitted to nominate two projects, one web series project, and one feature film (Telefilm, 2016a). In Ontario, for example, only Ryerson University, York University and Sheridan College are designated training institutions (Telefilm, 2016c). It is unclear why other institutions, for example, Humber College, are not also designated partners. According to the program guidelines, Telefilm does say that it will permit "flexibility" for partners to recommend projects from producers with whom they are not affiliated (Telefilm, 2016a), but it is unclear why a designated partner would be incentivized to do this, over forwarding individuals from their own communities. Thus, barriers exist already at the level of affiliation with the select industry partners. It is important to note also that while the Telefilm Micro-Budget Program is open to web series projects, it appears to 
prioritize films. In the last funding round, 14 feature film projects were selected for funding, while only four web series projects were successful (Telefilm, 2017).

All of the key funding programs open to web creators provide greater than $\$ 100$ 000 of maximum potential funding per project (including the Telefilm Micro-Budget Program). Throughout the interviews, many creators were adamant that a different approach should be taken in an effort to provide more opportunities to a greater number of potential creators. Interviewees thus felt that programs aimed at encouraging the entrance of new talent should provide smaller funding allocations to a greater number of projects and producers. Creators believed allocations of $\$ 10000$ would serve this purpose well, and would encourage more new voices to participate in a low-risk environment. Application requirements for allocations of this size should also be revised to reflect the lower level of risk involved on the part of the financier. With allocations this size, program-funding budgets could therefore be stretched much further to provide access points to more creators.

As mentioned previously, creators were also worried that as the funding system for web series becomes more institutionalized, it could contribute to the replication of patterns seen in television and film in the area of diversity and participation. As demonstrated by the results of the diversity study, this is a real concern. Furthermore, upon analysis, it appears that five of six of the inaugural batch of successful CMF 2017 Web Series Pilot Program projects were either directly supported by IPF for their prior 
season(s), or the applicant firm's prior projects were. This interconnected system, therefore, runs the risk of recreating a situation in which a small group of 'the usual suspects' will dominate the funding space. This possibility is why programs geared at encouraging and developing early-stage talent are so crucial, and why the establishment of a program which provides lower barrier, smaller-risk allocations to a greater number is a worthy consideration.

The envisioned program should decidedly be grant-based, and there should be no expectation for the recoupment of investment. It would be first and foremost a social strategy designed to develop new, diverse talent. Such a program could therefore allow more diverse, early stage creators to develop skills, test out ideas, build brands and grow audiences, and start on the path of developing the kinds of track records required to compete in existing and potential future funding programs that are aimed at achieving economic goals. An early career program such as this could also help creators become more attractive collaborators for larger scale, privately funded projects.

This kind of low-barrier, low-risk program would prevent web series from becoming homogenized, or as one creator was concerned, "a rich kid's medium", especially as having something to show increasingly becomes an expectation for new talent aiming at scripted screen careers in general. Such a program could therefore help to alleviate the homogeneity present in the wider scripted screen sector, as these 
early stage creators grow and develop their talents and enter the more professionalized space.

It must be noted as well that all of the funding programs, no matter what career stage they are targeting, should follow the IPF's lead in its new requirement for "knowledge-sharing" where projects led by those without web series experience must enlist the help of a mentor, while projects led by experienced web producers must install "new talent... with no more than one previous web series credit...in a key creative position" (IPF, 2017). For programs targeting the more advanced stage, installing a new talent in a key creative role might not be optimal, but incorporating them meaningfully as part of the team should be required nevertheless. It will be important to track whether the IPF's new requirement changes the outcomes discovered in this dissertation regarding the participation of women and visible minorities in key creative roles.

An early-stage program such as the one suggested here might also be strategically tailored to support creators specifically from traditionally marginalized groups, including women, visible minorities, Aboriginals, those with disabilities, those with limited economic means, immigrants, and individuals from the LGBTQ community. Furthermore, the goal of achieving parity and adequate representation levels should be extended to all Canadian public programs. While several Canadian public institutions, including Telefilm and the CMF have recently committed to gender parity 
in funding decisions, they have yet to commit to requirements related to the participation of other marginalized groups in programming (Zboralska, Davis, Shtern \& Ciccone, 2017).

iii. Growing Up the Talent

A comprehensive screen strategy that utilizes independent web series as an entry point for the incubation of early stage, diverse talent involves first encouraging new and diverse talent to enter the system by providing more equitable access points that acknowledge the continued centrality of socio-economic filters in the online world. The next component ensures that those who have been "trained up", either on their own, or through the existing or envisioned programs described above, have an industry and a market in which they can practice, and profit from, their talents and hard earned skills.

This is a seriously complex issue, and one that cannot be solved here. This is no doubt an area for future research and study. A comprehensive reimagining of the cultural support infrastructure in Canada will be necessary. Canada's federal programs aimed at the screen sector, will need to be realigned, ideally in concert with regional programs, to ensure that they are working to achieve the same goals. The role of each player in the system will need to be re-examined. As has been made clear in this work, and in numerous examinations of the issues in the past, broadcasters are often 
reluctant partners for Canadian creators, and over decades, have not been able to produce the kinds of consistent outcomes that are desired in a screen industry (audiences; critical acclaim; economic success).

The case for modifying the broadcast trigger system in CMF funding is thus strong. Broadcasters could be one trigger among many potential others (including foreign-owned firms) for funding. The successes of Canadian web series producers, which have been achieved without the budgets allotted to traditional television, and without traditional industry supports (including tax credits), are a testament to their passion and ingenuity. These creators should not be restricted from achieving further success. They should be eligible to collaborate with partners and distributors that seek excellence and innovation, rather than be restricted to those that have been found to treat Canadian content as a burden. Currently, web creators in the mid-tier whose project budgets are in the $\$ 200000-\$ 400000$ range are struggling to fit the scope of their visions into the budgets available to them. For many of them, their goal is not web series specifically, but for now, access to web series funding is more possible to attain than funding for convergent television projects with Canadian broadcasters. These creators and their firms are currently situated in a cruel purgatory in which they cannot graduate to the next level of production because the opportunities in scripted content in the Canadian screen industry are low. There is a great danger of losing the momentum that has been generated by these creators and their firms to brain drain to 
the United States. Several of executives at the higher-profile rising firms interviewed, admitted to having considered moving their operations to the United States. This is not something they desire, but they feel they may have no choice. In addition, funding at the advanced, professional tier should also be platform agnostic. Unlike the envisioned funding/ training program aimed at early stage talent, this tier of funding would primarily be concerned with the achievement of economic goals and the demonstration of market traction.

A reimagining of the system could begin with the diversion of funds spent on 'rich and substantive' digital media components for the CMF's Convergent stream to projects led by independent producers, as several of the web creators interviewed suggested. Other elements of Canada's funding system that will need to be evaluated include the federal and provincial tax credit system. While web series are now eligible for federal tax credits, they are, for example, not eligible at the provincial level in Ontario (Pinto, 2017). As demonstrated by the findings in this dissertation, there is also a need for funding programs aimed at web series specifically to move toward a shorter lifecycle to better reflect the fast-moving web space and the demands of online audiences. The IPF, CMF and Telefilm programs are all administered once per year. It should be noted that Creative Canada promises to reduce some of the bureaucratic hurdles associated with the provision of tax credits in the screen sector (Canadian Heritage, 2017). What the new programs will look like, remains to be seen. 
From the interviews, there also appears to be an urgent need for both more seed funding and operational support for cultural firms at the startup and early stage firm phase. Many creators either hoping to launch production companies aimed at digital content, or those with existing early stage firms, noted a lack of operational funding for their companies since existing supports are primarily project-based. Given the realities of the digital economy, and producers choosing their visions over taking their full fees, funding the 'company' side of their operations was an ongoing struggle for many. Seed and operational funding for startups and early firms could help to build more stable companies that can accomplish work more rapidly, and potentially, on a larger scale. A deep and comprehensive analysis of existing supports must be undertaken to evaluate redundancies, misalignments and areas of missed opportunity.

As part of the new cultural strategy, the government has opened up access to the Strategic Innovation Fund to firms in the creative industries (Canadian Heritage, 2017). This fund "is designed to spur innovation and aims, among other things, to encourage R\&D, facilitate the growth and expansion of firms, and advance development through collaboration between academia, non-profit organizations and the private sector" (Canadian Heritage, 2017, p.17). While this is a positive and welcome move, it is still unclear what effect this fund will have on creative firms wishing to expand, or whether emerging firms will be competitive in the program. 
Government also has a strong role to play in encouraging private investment in this area by facilitating and growing a culture of venture capital and angel investment in the Canadian creative industries. This would no doubt be a challenging endeavour, since, as is clear, investors prefer technology-oriented firms to purely cultural ones given the heightened chances for return on investment if a technology firm can scale (Duopoly, 2016). Cultural firms built for the future, however, require firm-level capabilities to use technology and data enhanced processes to develop, market, and distribute their content. There is potential for return on investment when looking in the longer term. New access to the Strategic Innovation Fund could potentially provide some of the capital required to build such future-oriented capacities in the absence of venture capital and angel investment in the cultural sector. If access to the fund enables firms to begin to develop deeper, data-driven capacities, perhaps this will have the additional effect of making Canadian cultural firms more attractive to private capital investment. Access to the fund might also encourage the development of new Canadian-owned distribution platforms and services, something this dissertation has shown to be greatly needed in Canada, particularly given the inconsistent interest in the development of online platforms and infrastructure by Canadian broadcasters.

To get at the issue of improving Canadian web producers' chances of accessing international streaming services, as one recent report suggests—Canada needs a national, concerted export strategy and "more funding to support promotion of 
Canadian television programs at major international markets and to encourage more Canadian companies to attend and build essential international relationships" (De Rosa \& Burgess, 2017). A concerted strategy, and increased funding to permit the development of international relationships could address some of the shortcomings identified throughout the interviews. The new cultural policy for Canada confirmed that the government will be moving precisely in this direction and will invest $\$ 125$ million over five years to "help Canadian creators achieve their international business objectives" (Canadian Heritage, 2017, p.28). Although the details are not yet known, this is a positive development for creative firms that struggle to connect with international buyers.

It is unclear whether Netflix's physical presence in Canada, as announced in the new cultural policy (Canadian Heritage, 2017), will increase its accessibility to independent Canadian creators. It is unknown whether Netflix will be interested in partnering with independent Canadian creators, who through their web series have shown immense potential (to grow and captivate audiences; to generate critical acclaim), or whether their partnerships will continue to be with broadcasters and firms that own established intellectual property. Whether Netflix will contribute to the "growing up" of Canadian talent, therefore, remains to be seen.

iv. Monitoring Diversity in Canadian Original Production 
If a central goal is to have more diversity in the Canadian screen system, how will we know we have achieved this goal unless diversity is consistently tracked and measured? Current monitoring of diversity by the CRTC and the federal government extends only to broadcasters, bypasses the entire independent production sector, and does not require reporting on key creative roles specifically (Zboralska, Davis, Shtern \& Ciccone, 2017). Academic researchers and industry interest groups fill in some gaps, but their efforts are mired by a lack of financial resources and time (Zboralska, Davis, Shtern \& Ciccone, 2017). Government resources should thus be devoted to such an initiative to ensure continuity, and the initiative should track participation across projects made for all screens.

Additionally, the new measures should aim at alleviating some of the challenges of tracking diversity in independent web series, as encountered in this research project, including the lack of a comprehensive, centralized database of web series, as well as the lack of access to data about the identity characteristics of key creative team members. A crowd-sourcing platform could be developed in which producers are incentivized in some way to provide the information whenever they produce a web series. Providing an avenue for individuals to self-identify their diversity characteristics (ex. gender, race, sexual preference, gender identity) would allow for a more firm and comprehensive picture of the status of diversity amongst web creators to emerge. 
v. Investing in Physical Infrastructure

The need for access to physical centres also exists, and this need is not being

served with the current media infrastructure. As one creator argued:

There needs to be a facility, why not have...essentially a YouTube creators' studio that's publicly funded? And everyone has access, so that not having access to equipment and resources and studio space [are no longer] your inhibitor. (Independent creator, James Ross)

As mentioned previously, YouTube spaces, which provide access to studio space, equipment and training are only open to YouTube partners with 10000 subscribers or more (YouTube, 2016b). At a recent CRTC hearing on the status of community television, there was avid support from various groups (CACTUS, 2015; IWCC, 2015; Making Media Public and the Communications Policy Working Group, 2015; The Community Media Policy Working Group, 2015) for the reimagining of the Canadian community media system so that it could encourage and cater to the kind of usage envisaged by creators throughout these interviews. In its submission, the IWCC argued:

Physical centres provide a centralized location for training, mentoring and the production and trade of ideas. Physical production centres also lead to higher production values in programming as they facilitate necessary equipment such as video, lighting, sound mixing, broadcast, and internet resources... While the cost of digital equipment is dramatically lower than it was even ten years ago, it is still out of reach of a significant portion of the Canadian population. Having physical facilities provides a means of including communities that have traditionally been underrepresented in the Canadian broadcasting system. It also ensures consistent access to resources that is not dependent on an individual community member or creator's own personal financial situation, which may fluctuate. (IWCC, 2015) 
Despite a clear desire by many for an updated community media system that could encourage participation by easing barriers, the CRTC decided instead to allow the majors, which largely control the community media system, to shutter community media studios in major markets in order to divert funds to subsidize struggling local news stations (CRTC 2016-224). The decision should never have been framed as a dichotomy, and many hearing participants were frustrated by the way the issue was presented and structured. Since the decision has been made, community media studios across many Canadian cities have been shuttered (Hunter, 2017). And while YouTube clearly recognizes the value in physical spaces for its own creators, the CRTC, in its decision, overlooked their importance. As part of the government's new cultural strategy, it is investing $\$ 300$ million over 10 years to develop new physical, cultural spaces including incubators and co-working spaces (Canadian Heritage, 2017). It is unclear whether any of these new "creative hubs" (Canadian Heritage, 2017) will contain the kind of production spaces and equipment independent web creators could use.

vi. Business and Skills Training

There is also a strong need, and indeed desire for, business, entrepreneurial and digital training amongst the creators interviewed, or connection to experts who could fulfill the roles required. The newly announced creative hubs could become access 
points for such training, and must be designed to provide this training at an affordable cost, and at times amenable to the creator community, whose schedules often prohibit extracurricular activities at certain points throughout the year. Making such affordable training available could immensely strengthen Canadian creators' chances for success. Indeed, creators need to be exposed to new ideas and services. For example, growth hacking, or using affordances enabled by digital technologies like automation to grow user bases exponentially despite small marketing budgets (Holiday, 2014), could be immeasurably useful to the creator community. As Canada's traditional jobs continue to be disrupted by technology, new ones, such as 'Audience Growth Hacker' could replace them. The digital-first screen sector could help create demand for new jobs, and in turn, increase odds for its success.

As the dissertation revealed, web series are creating demand for news kinds of expertise, including online community builders, social media experts, crowdfunding strategists, design-thinkers and more. Funding programs could thus require successful projects to earmark a certain proportion of their funding to the hiring of such experts. The Telefilm Micro-Budget Program already requires successful applicants to hire someone to provide digital marketing and/or social media expertise (Telefilm, 2016a). The issue is, however, there appears to be a dearth of individuals who possess these skills. Universities, colleges and other training institutions should review existing programs to ensure they are producing graduates with the kinds of skills required. A 
single web series production involves the collaboration of a wide array of competencies. Educational institutions could thus create collaborative courses designed around the creation, launch and marketing of a single web series, that would be open to, and indeed require, the participation of students from a disparate range of disciplines. This would not only ensure the development of contemporary, in-demand skills, but, with all roles being filled, and under the guidance of an expert, could lead to potentially innovative and impactful online content.

\section{vi. Evaluating Existing Production Agreements}

Another area for re-evaluation is the current ACTRA new media agreement. While it is without a doubt that the union has the best of intentions, the findings from the interviews demonstrate that the current rate structures have placed union actors out of reach of many independently produced Canadian web series. As previously discussed, the current pricing structures may be impeding the advancement of the nascent web series space, encouraging the migration of web series production to the United States where web series performer rates are negotiable under SAG-AFTRA rules, and limiting opportunities for performers to practice their craft in meaningful roles which are rarely available.

In 2017, for web series with budgets of $\$ 7500$ per minute and below (which covers virtually all independently produced Canadian web series at the moment), a 
single principal actor rate is $\$ 470.76$ per day (ACTRA, 2016). In the US, under SAGAFTRA, the rates for principal performers working on web series with budgets below $\$ 66112.50$ CAD per episode (\$50 000 USD) (virtually all Canadian independently produced web series would fall under this), are completely negotiable (SAG-AFTRA, personal communication, email, May 16, 2017). A rate of \$165.31 CAD (\$125 USD) applies for productions with budgets between $\$ 66112.50$ CAD per episode ( $\$ 50000$ USD) and $\$ 330612.50$ CAD (\$250 000 USD) (SAG-AFTRA, personal communication, email, May 16, 2017). Thus, even at this comparably high budget level, the SAGAFTRA daily rate is significantly lower than ACTRA's lowest budget tier.

ACTRA should thus consider the creation of two (at least) new budget tiers so that it better reflects the realities of the economics of the independent digital content ecosystem. The first new tier should be an ultra low to no budget tier that would permit performers to be able to negotiate their rates, and to accept no payment, in web series under a certain budget threshold as is the case in the US; the second new tier should be for web productions receiving funding from programs like the IPF, CMF or Telefilm, with budgets (from all sources) up to approximately $\$ 400000$. The new tier should allow for a performer rate that is at minimum, half of the current rate for the existing lowest tier (i.e. reduce, at minimum, from $\$ 470.76$ per day to $\$ 235.38$ ). This would leave producers with these modest budgets with some additional runway for hiring more crew members, experts and marketing their series, which would not only enhance 
their projects' chances for success, but also, increase the profile of the performers hired. As it is now, Canadian web series producers are stretched far too thin and chances for success are not being maximized.

vii. An Expanded Role for the Public Broadcaster

Finally, there is an increasing role for Canada's public broadcaster to play in the commissioning and dissemination of Canadian content. There is clearly a need for a distribution platform that champions Canadian content, centralizes it, and makes it easily accessible to audiences. The $\mathrm{CBC}$ has recently announced that it will soon be launching a new OTT product (Maloney, 2017). Similar to the Britain's main public broadcaster, the $B B C$, the $C B C$ could sell this service to international audiences for added revenue. The envisioned CBC international OTT product could be a Netflix-like service that sells a newly revamped, competitive Canadian content brand globally. Such a product would require considerable resources, a commitment to stay the course, an understanding that return on investment will take time, and of course, bold leadership.

\section{Future Work}

This section has provided a blueprint of priority areas for policy and industry stakeholders to address the issues identified in this dissertation. The recommendations identified here aim at encouraging the positive outcomes Canadian web creators have 
been able to achieve in a bid to strengthen the Canadian scripted screen sector at large. If strategically designed and implemented along the lines described, policy and programs for independent web series can provide an antidote to some of the negative externalities that have emerged from cultural policy in traditional television.

There is ample work left to be done in this area. In addition to the future possibilities for research outlined throughout this chapter, further avenues that simply could not be explored here due to limitations related to both time and resources include a wider examination of diversity in key roles in web series receiving funding from programs beyond the IPF; an examination of on-screen diversity in Canadian web series to explore whether stories and characters are qualitatively and quantitatively more diverse than those seen in traditional television; an examination of the state of Canadian unscripted web series creation conducted in a similarly systematic way; an examination of the diversity of creators working in Canada's interactive content sector including gaming and VR; an examination of the commissioning practices of the major transnational streamers (who do they hire, and what do they fund?); a study with a longitudinal component that would examine the progress of Canadian web creators' careers over time; and the development and execution of a pilot collaborative course that would bring together students from disparate disciplines in the way described. 


\section{Appendices}

\section{0 - Interview Protocol (Creators)}

TELLING OUR STORIES ON THE WEB: CANADIAN ENGLISH-LANGUAGE WEB SERIES AND THE PRODUCTION OF CULTURE ONLINE

[DATE]

[NAME OF PARTICIPANT]

A. Background Information:

- Age:

- Gender:

- Education: (Formal education- college diploma; undergraduate degree; graduate school)

- Training: (Informal and formal mentorship; vocational training)

- Visible Minority: (Yes/ No)

- Ethno-cultural background:

- First Generation: (Yes/ No)

- Mother tongue:

- Disability status: (Any diagnosed disabilities)

- Family background: low-income, middle-class, upper-class (Household annual income: Below $\$ 25$ 000; $\$ 25000$ - \$50 000; $\$ 50000$ - \$75 000; \$75 000 - \$100 000; Above $\$ 100000$ )

B. Career:

- Describe your career history. Do you have a day job? Have you worked in traditional television before?

C. Identity \& Motivation:

- Provide a brief plot summary of your most recent web series.

- What is your motivation for making scripted stories for the web rather than traditional TV?

- What was the inspiration for your web series and its particular subject matter? Why do you tell the stories that you do? 
- Do you feel that you, or the kinds of stories that you want to tell, have been marginalized by the legacy system, its structure, and its main actors? Why and how?

- What goals do you have for your web series? Economic? Societal? Other? To be picked up by a mainstream broadcaster? To be picked up by online distributor?

- How much autonomy do you have over the kinds of stories that you are able to tell and in the way you tell them? How do you determine your limits for pushing the envelope?

- Traditional industry barriers: Do you feel that any of the following attributes/ issues present challenges or barriers that affect your ability to engage in web series production?

Age

Gender

Race

Sexuality

Class

Education

Culture

Skills/ training/ talent development

Networking/ mentorship

Ability/ disability

Other

- Do you believe that your web series is different in story from the web series being produced by legacy broadcasters, and content produced for legacy media? Why or why not?

- Have you ever applied for funding? If yes, have you ever changed your story, or tailored it to be eligible for funding? Have you ever compromised your artistic vision to be eligible for funding? Have you ever received funding?

- Do you identify as an entrepreneur (i.e. a person who creates, defines, discovers, and exploits opportunities)? Why or why not?

- Do you want to establish a production company? Where do you want to be in 5 years?

D. Audience:

- When producing for the web, what is the target market for your products (niche global audience; niche Canadian audience; global mainstream audience; mainstream Canadian audience; other)? 
- How easy or difficult is it to find niche audiences on the web? Is niche content or traditionally under-produced content amenable to distribution on the web from an economic perspective?

- Would you, or have you, ever included identifiable Canadian-specific elements into a web series, or told a Canadian-specific story on the web? Why or why not?

G. Financing, Revenue \& Success:

- What is the per-season budget of your web series?

- How do you finance your web series?

- Have you monetized your web series? If so, how?

- How much total revenue has your web series generated to date? What are your average monthly revenues? Broken even? Average views per episode?

- Do you consider your web series to be successful? What barriers stood in the way of its (further) success?

- Has your web series won any awards?

F. Production:

- What package of skills is needed to succeed in creating successful web series? Are these skills different from the skills needed to create for traditional television?

- What are major trends in the web series industry in relation to content, form, financing and monetization? How do you become aware of trends in this space or, how do you create new trends?

- How does the medium of the web change the form of your content in comparison to content made for television? How about the genre or orientation?

- How do you choose your distribution platform (ex. sell to online video service such as Netflix vs. create a discrete website vs. upload to video hosting site such as YouTube or Vimeo)?

- Do you use data or other digital audience engagement metrics/ ratings in the production of your content? If so, which ones? Have you ever changed/adjusted storylines or characters based on this data?

- How do you approach casting for web-first productions in light of the potentially global audience? How important are considerations of gender, age and race when casting for a potentially global market? Is this the same or different than when casting for traditional television?

H. Labour:

- When working on a web series, what are working conditions like? Rates of pay? Roles and duties? Did you pay your cast and crew on your last web series? 
- Are there any new roles that have emerged due to the nature of producing content for the web rather than traditional television? Have any roles become redundant? Have any roles converged?

I. Politics, Power and Policy:

- What are the top 5 factors that can make or break a web series?

- What does the term, "web series", mean to you? What kind of connotation does it have?

- Describe the web series community. Who makes web series?

- What's your favourite scripted web series (from any country)?

- Are there any gatekeepers in this industry, and if so, who are they?

- What's the future of television?

- Is there a rationale for the public funding of web series? If yes, what is it?

- What gaps do you see with respect to the way online content is funded in Canada, and the way web talent is developed? What policies would improve Canadian creators' opportunities for success in the online space in relation to any of the following areas: research and development, production and financing, training and skills development, promotion and discoverability, distribution and monetization?

- What lessons does Canada's lack of diversity both on and behind the screen in legacy media (ex. few women, few visible minorities etc.) teach us about the industry and how do you think these lessons should be applied to the new media audiovisual industry? Do you see any potential solutions (ex. ideas for programs, policies etc.)?

- What lessons does Canada's lukewarm success in the development of scripted series for legacy media teach us about the industry and how do you think these lessons should be applied to development for the web?

- Are you part of any industry guilds, or trades or labor organizations? Why or why not? Do you believe that collective bargaining is important for web creators?

Would you like to receive updates by email about study outcomes? 


\section{1 - Interview Protocol (Majors)}

TELLING OUR STORIES ON THE WEB: CANADIAN ENGLISH-LANGUAGE WEB SERIES AND THE PRODUCTION OF CULTURE ONLINE

[DATE]

[NAME OF PARTICIPANT]

[COMPANY]

A. Internal Company Division and Change Management:

- What are the current opportunities and challenges of pursuing a web-first strategy in Canada for someone in your position? What is the current status of the market for Canadian web series in Canada and globally?

- What is the division of duties in your company with respect to web-shows/ digital content (other than audiovisual - ex. games and apps) vs. traditional content? Are there separate departments/ teams? Could you describe the division of labour in the company in relation to digital content?

- How would describe your company's allocation of resources directed at episodic web-shows, non-episodic audiovisual web-content (value added content), digital content other than web-shows (ex. games and apps), and traditional television?

- What do you believe television will look like in 10 years? Will it be delivered via broadband or cable/ satellite?

- What is your company's 5-year plan with respect to the way it allocates resources to audiovisual content creation for the web, and for traditional television?

- What is your company's strategy for change management?

- Are there tensions within the company's various departments/ assets with respect to how to approach change brought on by digital convergence?

- How does revenue from your online ventures (web-shows only, and all digital ventures combined) compare to revenue from traditional television?

- With respect to original content, how does your company decide which stories will be for the web, and which will be for traditional television?

- Do you have more autonomy when developing or overseeing the development of a web property, than when developing properties for television?

- How do you rate your company's Entrepreneurial Orientation along the following key dimensions in relation to its approach to digital: 1) autonomy 2) innovativeness 3) risk-taking 4) pro-activeness and 5) competitive aggressiveness? 
- In your view, can and will, Canadian broadcasters survive in the new media landscape by relying on the historical model of acquiring hit foreign-American programming? Why or why not?

\section{B. Production}

- What are major trends in the web series industry in relation to content, form, financing and monetization? How do you become aware of trends in this space or, how do you create new trends?

- Are most scripted and unscripted episodic web-shows that your company produces produced in-house, acquired, or produced in conjunction with individual creators or independent production companies? How do you acquire/ develop/ web-shows?

- Is there anything unique/ different about your web-shows vs. your traditional shows? Do you have more freedom in this space to tell the kinds of stories that you want to tell?

- For partnerships-how much oversight do you have over the development/ production process? How much autonomy do you give the independent producers/ production companies when in development/ production on a web series, versus a property being developed/ produced for traditional television? Why?

- Are the skills needed to succeed in the development and production of a webshow different from the skills needed to produce for traditional television?

- How does the medium of the web change the form of your content in comparison to content made for television?

- Do you use data or other digital audience engagement metrics/ ratings in the production of your content? If so, which ones? Have you ever changed/adjusted storylines or characters based on this data?

- How does casting for web-first productions differ from casting for traditional projects in light of the potentially global audience? How important are considerations of gender, age and race when casting for a potentially global market?

C. Audience:

- When developing scripted audiovisual content for the web, what is the target market for your content (niche global audience; niche Canadian audience; global mainstream audience; mainstream Canadian audience; other)?

- How easy or difficult is it to find niche audiences on the web? Is niche content or traditionally under-produced content amenable to distribution on the web from an economic perspective? 
- How does producing content specifically for the online space affect the genre, quality and orientation of content that is produced?

- Would you, or have you, ever included identifiable Canadian-specific elements into a scripted web series, or told a Canadian-specific story on the web? Why or why not?

- Is Canada's small market problem solved by distribution over the web?

D. Financing and Revenue:

- What is the per-season budget of a typical episodic, scripted web-show produced by your company? Or, how much has your company spent on scripted web series in the last year?

- How do you finance your company's web-shows?

- Have you ever applied for funding? If so, what kind of funding? Were you successful?

- Have you monetized your web-shows? If so, how?

- How do you gauge that your web-show has been a success? Economic return? Critical acclaim? Ratings? Other?

E. Labour:

- How does the division of work and labour in a web production compare to the division of labour on a traditional production aimed for television? Rates of pay? Roles?

- Are there any new roles that have emerged due to the nature of producing content for the web rather than traditional television? Have any roles become redundant? Have any roles converged?

F. Politics, Power and Policy:

- What lessons does Canada's lukewarm success in the development of scripted series for legacy media teach us about the industry and how do you think these lessons should be applied to production for the web?

- Does the vertical integration of most Canadian broadcasters present a challenge to innovation, and their ability to fully enter and engage in the online space?

- Is there a role for cultural policy (ex. the Broadcasting Act) in the online space? Why or why not?

- What lessons does Canada's lack of diversity both on and behind the screen in legacy media (ex. few women, visible minorities etc.) teach us about the industry and how do you think these lessons should be applied to the new media audiovisual industry? 
- Should production of Canadian scripted audiovisual content online be publicly funded? Why or why not? Should there be separate funding for individual vs. production company produced, or major broadcaster produced, online content, or should all the money go into one pool? Why?

- Do you view the public funding of cultural production as detrimental to, or beneficial for, entrepreneurship and innovation?

- Currently, there are rules in place that require a certain portion of Canadian content to be independently made (i.e. not made in house by major broadcasters such as Rogers, Bell, Shaw and etc.)—should this be applied to the online space? Why or why not?

- Do you believe there is cultural or societal value in ensuring that there is Canadian-produced scripted content aimed at domestic Canadian audiences in the online space (i.e. content prone to market failure)?

- Do you believe that there should be any policy mechanisms or programs put in place to foster the growth of an economically sustainable, inclusive and innovative web series industry (example_funding mechanisms; professional mentorships etc.)?

Would you like to receive updates by email about study outcomes? 


\subsection{Interview Protocol (Policymakers/ Specialists)}

TELLING OUR STORIES ON THE WEB: CANADIAN ENGLISH-LANGUAGE WEB SERIES AND THE PRODUCTION OF CULTURE ONLINE

[DATE]

[NAME OF PARTICIPANT]

[ORGANIZATION]

A. For Funding and Development Agencies

- Describe your current funding or development programs for episodic, standalone web-shows (both scripted and unscripted). Do these programs require broadcaster commitments?

- Do you believe there are any gaps in the current funding schemes/ development programs that exist?

B. For Guilds \& Trades Organizations

- What new opportunities have been created for your constituents because of the web? What are the challenges associated with this area?

- What systematic challenges or barriers exist for your constituents who produce for the web or want to produce in this space in relation to any of the following?

Age

Gender

Race

Sexuality

Class

Religion

Education

Culture

Skills/ training/ talent development

Networking/ mentorship

Personality traits

Changing technologies

Ability/ disability

Other 
- Do you believe collective bargaining is important for web creators?

\section{All Policy Actors}

- What do you believe television will look like in 10 years? Will it be delivered via broadband or cable/ satellite?

- What lessons does Canada's lukewarm success in the development of scripted series for legacy media teach us about the industry and how do you think these lessons should be applied to production for the web (in relation to the way the industry is structured, and/ or how content is funded/ developed, for example)?

- What lessons does Canada's lack of diversity both on and behind the screen in legacy media (ex. few women, few visible minorities etc.) teach us about the industry and how do you think these lessons should be applied to the new media audiovisual industry?

- What policies would improve Canadian creators' opportunities for success in the online space in relation to any of the following areas: research and development, production and financing, training and skills development, promotion and discoverability, distribution and monetization?

- What policies are needed to ensure equal access to opportunities in the online space?

- Do you have any suggestions for policies that would ensure the long-term development and success of new media entrepreneurs who have showed promise in the online space? Currently, no such policies exist. Would such policies be useful?

- What policies are required to ensure the diversity of Canadian professional web content, including uniquely Canadian stories, and those with more universal appeal in the online space?

- Do any of the historical justifications for the regulation/ subsidization of the broadcasting system (small market, market failure for niche content, lack of funding) apply to audiovisual entertainment in the online space?

- Is a thriving Canadian production industry likely if nothing is changed in the policy framework and funding mechanisms available?

- Does the legislative division between broadcasting and telecommunications make sense in the current era?

- Do you believe the vertical integration of most Canadian broadcasters present a challenge to innovation, and their ability to fully enter and engage in the online space? What can/ should be done about this?

- Should production of Canadian scripted audiovisual content online be publicly funded? Why or why not? Should there be separate funding for individual vs. 
production company produced, or major broadcaster produced, online content, or should all the money go into one pool? Why?

- Do you view the public funding of cultural production as detrimental to, or beneficial for, entrepreneurship and innovation?

- Currently, there are rules in place that require a certain portion of Canadian content to be independently made (i.e. not made in house by major broadcasters such as Rogers, Bell, Shaw and etc.)—should this be applied to the online space? Why or why not?

- Do you believe there is cultural or societal value in ensuring that there is Canadian produced scripted content aimed at domestic Canadian audiences in the online space (i.e. content prone to market failure)? Do you believe such content is economically feasible in the online world?

Would you like to receive updates by email about study outcomes? 


\subsection{List of Web Series Included in Quantitative Diversity Analysis}

\begin{tabular}{|c|c|}
\hline 1. & 13 Witches \\
\hline 2. & 1800 Dirty Talk \\
\hline 3. & 2012 the Webseries S. 1 \\
\hline 4. & 2012 the Webseries S.2 \\
\hline 5. & A Day in the Life of Death \\
\hline 6. & A Full Rotation \\
\hline 7. & All Fair's Play \\
\hline 8. & Almost a Turkish Soap Opera \\
\hline 9. & Anarkali \\
\hline 10. & Ariel Erisian \\
\hline 11. & Asset \\
\hline 12. & Axe Lords \\
\hline 13. & Bad Seeds \\
\hline 14. & Ben Johnson Vlog \\
\hline 15. & Best Before Show \\
\hline 16. & Bike Cop: The Series \\
\hline 17. & Bill \& Sons Towing \\
\hline 18. & Bill and Sons Towing \\
\hline 19. & BJ Fletcher: Private Eye S.1 \\
\hline 20. & BJ Fletcher: Private Eye S.2 \\
\hline 21. & Blank Verse \\
\hline 22. & Bob and Andrew S.1 \\
\hline 23. & Bob and Andrew S.2 \\
\hline 24. & But I'm Chris Jericho \\
\hline 25. & Carmilla the Series Prequel \\
\hline 26. & Carmilla the Series S.1 \\
\hline 27. & Carmilla the Series S.2 \\
\hline 28. & Chad's Angels \\
\hline 29. & Claddagh The Series S.1 \\
\hline 30. & Claddagh The Series S.2 \\
\hline 31. & Clutch: The Series S.1 \\
\hline 32. & Clutch: The Series S.2 \\
\hline 33. & Coffee With Pterodactyls \\
\hline 34. & Couple-ish \\
\hline 35. & Cut to the Chase \\
\hline 36. & Davey's Diary \\
\hline 37. & Dhaliwal '15 \\
\hline
\end{tabular}


38. Divine the Series

39. Everyone's Famous

40. Fame and Fidelity

41. Fools For Hire S.1

42. Fools For Hire S.2

43. Found Viral

44. Gay Nerds S.1

45. Gay Nerds S.2

46. Golden Futures

47. Goodbye Sara Henessey

48. Guidestones S.1

49. Guidestones S.2

50. Haphead

51. Heroes of the North

52. Hitman 101

53. How to be an Actor

54. In Counseling

55. In the Rough

56. Insayshable

57. Jigsaw the Series

58. Job Review with a Vampire

59. Just Passing Through

60. Kate Conway is a Jerk

61. Kids Town

62. Kirby in Therapy

63. Kits these days

64. LARPS the Series S.1

65. LARPS the Series S.2

66. Last Chance Casting

67. Last in Space

68. LESlieVILLE

69. Less than Satisfactory

70. Libelle

71. Long Story, Short

72. Machiavelli's The Prince

73. March Family Letters

74. Masculathon

75. Microwave Porn

76. Millions the Series 
77. Minds Eye the Series

78. Model Minority

79. Moderation Town S.1

80. Moderation Town S.2

81. Ninjas

82. On the Heated Floor

83. Out with Dad S.1

84. Out with Dad S.2

85. Out with Dad S.3

86. Out with Dad S.4

87. Papillon

88. Parked

89. Pay Up

90. Pete Winning and the Pirates S.1

91. Pete Winning and the Pirates S.2

92. Please Don't Fire Me

93. Poopsie Dries Out

94. Preflight Launch S.1

95. Preflight Launch S.2

96. Pretty In Geek

97. Prison Dancer

98. Riftwood Chronicles

$99 . \quad$ Roomies

100. Royal Bitch

101. Ruby Skye P.I. :Spam Scam

102. Ruby Skye P.I.: The Haunted Library

103. Ruby Skye P.I.: The Maltese Puppy

104. S.O.S. Save Our Skins

105. Season of the Plague

106. Secret Diary of a Call Centre Girl

107. Sexy Nerd Girl

108. Single and Dating in BC S.1

109. Single and Dating in BC S.2

110. Solilioquies of Santiago

111. Someone Not There

112. Space Janitors S.1

113. Space Janitors S.2

114. Space Janitors S.3

115. Space Riders 
116. Spell Fury S.1

117. Spell Fury S.2

118. Squared and Uncocked

119. Standard of Action S.1

120. Standard of Action S.2

121. Standard of Action S.3

122. Stink

123. Straight Man

124. Sweet Fever

125. Sweet Tarts Takeaway

126. Talking Up

127. Team Leader

128. Teenagers S.1

129. Teenagers S.2

130. The Actress Diaries S.1

131. The Actress Diaries S.2

132. The Amazing Gayl Pile

133. The Autobiography of Jane Eyre

134. The Burden of Genius

135. The Casting Room S.1

136. The Casting Room S.2

137. The Casting Room S.3

138. The Casting Room S.4

139. The Court Supreme

140. The Crapsville

141. The Duchamps School of Acting

142. The Fall

143. The Gate Series

144. The Giant Comes Out

145. The Last Fall of Ashes

146. The People That Touch Your Food S.1

147. The People That Touch Your Food S.2

148. The Plateaus

149. The Raiders Session

150. The Runner

151. The Spell Tutor

152. The True Heroines

153. The Young and Undead

154. Through the Copy Glass 
155. Tights and Fights: Ashes

156. Time Barristas

157. To Punchy

158. Unlikely Heroes

159. V Morgan is Dead

160. Venus Spa S.1

161. Venus Spa S.2

162. Venus Spa S.3

163. Verdict

164. Versus Valerie

165. Vita Bella: The Dogumentary

166. Wasted Time S.1

167. Wasted Time S.2

168. Whatever, Linda

169. White Collar Poet

170. Who The F\#\#k Is Nancy?! S.1

171. Who The F\#\#k Is Nancy?! S.2

172. Yid Life Crisis S.1

173. Yid Life Crisis S.2

174. Yoga Town

175. Your Lupine Life 


\section{References}

ACTRA (2013). Independent Production Agreement January 1, 2013, to December 31, 2015. Retrieved http://cmpa.ca/sites/default/files/documents/IR/2013\%20\%2015\%20ACTRA\%20IPA.pdf

ACTRA (2016). Independent Production Agreement January 1, 2016, to December 31, 2018.

ACTRA (n.d.) Resources - Co-op. Retrieved on 15 May 2017 http://www.actratoronto.com/performers/my-union/committees/resources-co-op/

Allen, K., Quinn, J., Hollingworth, S., \& Rose, A. (2013). Becoming employable students and 'ideal' creative workers: exclusion and inequality in higher education work placements. British Journal of Sociology of Education, 34(3), 431-452.

Amit, R. \& Muller, E. (1995). "Push and Pull" entrepreneurship. Journal of Small Business and Entrepreneurship, 12(4), 64-80.

Amit, R., MacCrimmon, K., Zietsma, C. and Oesch, J.M. (2001), Does money matter? Wealth attainment as the motive for initiating growth-orientated technology businesses. Journal of Business Venturing, 16(2), 119-43.

Analysis Group. (2012). Vertical Integration in TV Broadcasting and Distribution in G8 Countries and Certain Other Countries. (Rep.). Analysis Group.

Anand, N. and BC. Jones. (2008). Tournament rituals, category dynamics, and field configuration: The case of the Booker Prize." Journal of Management Studies 45, 1036-1060.

Andreeva, N. (2016 April 20). Whatever, Linda' web series to get TV adaptation by 'Orphan Black' co-creator \& Mark Gordon Co. Retrieved http://deadline.com/2016/04/whatever-linda-web-series-tv-series-mark-gordoncompany-hannah-cheesman-1201741078/

Andrejevic M (2009) Exploiting YouTube: Contradictions of user-generated labor. In: Snickars P and Vonderau P (Eds.), The YouTube reader. Stockholm: National Library of Sweden, pp. 406-423. 
Antcliff, V., Saundry, R., \& Stuart, M. (2007). Networks and social capital in the UK television industry: The weakness of weak ties. Human Relations, 60(2), 371-393.

Apitzsch, B. (2010, January). Informal networks and risk coping strategies in temporary organizations: the case of media production in Germany. In Forum Qualitative Sozialforschung/Forum: Qualitative Social Research (Vol. 11, No. 1).

Arias, A. \& Pena, I. (2010). The effect of entrepreneurs' motivation and the local economic environment on young venture performance. International Journal of Business Environment, 3(1), 38-56.

Armstrong, R. (2010). Broadcasting policy in Canada. University of Toronto Press.

Ashton, K.L. (2017). Tweet. 11:45 AM - 2 February, https://twitter.com/klashton27/status/827181100619423746

Babe, R. E. 1990. Telecommunications in Canada. Toronto: U of T Press.

Barnard, L. (November 9, 2013 Saturday ). Going beyond viral; YouTube comes of age with Buffer Festival, showcasing site's content, online stars. The Toronto Star, Retrieved from www.lexisnexis.com/hottopics/Inacademic

Baron, A. (2013). The death, destruction, \& rebirth of the online video ad market. Retrieved from http://www.tubefilter.com/2013/10/21/the-death-destructionrebirth-of-the-online-video-ad-market/

Baudrillard, J., \& Foss, P. (1983). Simulations (p. 1). New York: Semiotext (e).

Beggs, J., Doolittle, D., \& Garsombke, D. (1994). Entrepreneurship Interface: Linkages to Race, Sex, and Class. Race, Sex \& Class, 1(2), 35-51. Retrieved from http://www.jstor.org/stable/41680220

Bell Media (2015, November 9). Massive friggin' news, bud: CraveTV's first-ever original series Letterkenny to launch Super Bowl Sunday, February 7 Retrieved http://www.bellmedia.ca/pr/press/cravetvs-first-ever-original-series-letterkennylaunch-super-bowl-sunday-february-7/

Bell Media (2016, June 7). CraveTV taps comic megastar Russell Peters for next original series, Russell Peters is the Indian Detective. Retrieved here 
http://www.bellmedia.ca/pr/press/cravetv-taps-comic-megastar-russell-peters-fornext-original-series-russell-peters-is-the-indian-detective/

Benkler, Y. (2006). The wealth of networks. New Haven, CT: Yale University Press.

Bergmann, H. \& Sternberg, R. (2007). The changing face of entrepreneurship in Germany. Small Business Economics, 28(2-3), 205-221.

Berkowitz, I. (2016). Domestic supply to global demand: Reframing the challenge of Canadian English-language television drama. Dissertation. Ryerson University.

Bhola, R., Verheul, I., Thurik, R. \& Grilo, I. (2006). Explaining engagement levels of opportunity and necessity entrepreneurs. EIM Business and Policy Research, H200610.

Bird, S. E. (2011). Are we all produsers now? Convergence and media audience practices. Cultural Studies, 25(4-5), 502-516.

Blais, J.P., (2013, June 12). Speech by Jean Pierre Blais at Banff World Media Festival. Speech presented at Banff World Media Festival, Banff. Retrieved from http://www.crtc.gc.ca/eng/com200/2013/s130612.htm

Blinning, C. (2010). The showrunner model of production. Canadian Screenwriter. Summer 2010. Retrieved: http://cdnscreenwriter.dgtlpub.com/2010/2010-0531/home.php?page_view $=10$

Block, J. \& Sandner, P. (2009). Necessity and opportunity entrepreneurs and their duration in self-employment: Evidence from German micro data. Journal of Industry, Competition and Trade, 9(2), 117-137.

Block, J. H., \& Wagner, M. (2010). Necessity and opportunity entrepreneurs in Germany: Characteristics and earnings differentials. Schmalenbach Business Review, 62, 154-174.

Bloor, M., \& Wood, F. (2006). Ethnography. In Keywords in Qualitative Methods. (pp. 70-76). London, England: SAGE Publications Ltd. doi: http://dx.doi.org/10.4135/9781849209403.n21

Bock, A., Isermann, H., \& Knieper, T. (2011). 14 Quantitative Content Analysis of the Visual. In E. Margolis, \& L. Pauwels (Eds.), The SAGE Handbook Visual Research 
Methods. (pp. 265-283). London: SAGE Publications Ltd. doi:

http://dx.doi.org/10.4135/9781446268278.n14

Booba1234. (2009 January 30). David after Dentist. [Video file]. Retrieved

https://www.youtube.com/watch?v=txqiwrbYGrs

Bourdieu, P. (1993). The field of cultural production: Essays on art and literature. New York: Columbia University Press

Brettel, M., Engelen, A., \& Heinemann, F. (2009). New entrepreneurial ventures in a globalized world: The role of market orientation. Journal of International Entrepreneurship, 7(2), 88-110.

Brownell, C. (October 18, 2014). Monetizing YouTube; Content providers blaze golden trail to prosperity. National Post, Retrieved from www.lexisnexis.com/hottopics/Inacademic

Bunche. (2014). 2014 Hollywood diversity report: Making sense of the disconnect. (Rep.). Retrieved http://www.bunchecenter.ucla.edu/wpcontent/uploads/2014/02/2014-Hollywood-Diversity-Report-2-12-14.pdf

Butler, M. (2016). Net-works: Collaborative modes of cultural production in Web 2.0 contexts. In Butler, M., Hausmann, A., and Kirchhofer, A. (eds.), Precarious alliances: Cultures of participation in print and other Media. Verlag, Bielefeld: Transcript.

Buttner, H.E. \& Moore, D.P. (1997). Women's organizational exodus to entrepreneurship: Self-reported motivations and correlates with Success. Journal of Small Business Management, 35 (1), 34-46.

Caldwell, J.T. (2008). Production culture: Industrial reflexivity and critical practice in film and television. Duke University Press.

Caldwell, J.T. (2009). Cultures of production: Studying industry's deep texts, reflexive rituals, and managed self-disclosures. In Holt, J., \& Perren, A. (Eds.) Media industries: History, theory, and method. West Sussex, United Kingdom: WileyBlackwell.

Canada, Department of Justice. (1991, February 1). Broadcasting Act 1991, C. 11. Retrieved from http://laws.justice.gc.ca 
Canada, (2016). Focusing the conversation: Consultation paper. Retrieved

https://www.canadiancontentconsultations.ca/system/documents/attachments/e32 8d01aaa5d8b25b5b2e769f0f3ccb59f63893e/000/004/022/original/PCH-

DigiCanCon-Consultation_Paper.pdf_

Canada Media Fund (CMF). (2017a). Performance Envelope Program Guidelines. 20172018. Retrieved http://www.cmf-fmc.ca/getattachment/6f543af0-769e-46ca-a9a99722658125e9/Performance-Envelope-Program-Guidelines.aspx

Canada Media Fund (CMF). (2017b). Experimental Stream- Web Series Pilot Program guidelines. Retrieved http://www.cmf-fmc.ca/getattachment/60be2081-32df-4147afe3-1fda3d54abac/Web-Series-Pilot-Program-Guidelines.aspx

Canada Media Fund (CMF). (2016a). 2015-2016 annual report. Retrieved https://arra15-16.cmf-fmc.ca/audience/audience_results

Canada Media Fund (CMF). (2016b). Profile 2015: Economic report on the screenbased media production industry in Canada. Retrieved http://www.cmpa.ca/sites/default/files/Profile\%202015\%20\%28English\%29.pdf

Canada Media Fund (CMF). (2015). Performance Envelope Program Guidelines 20152016. Retrieved from http://www.cmf-fmc.ca/documents/files/programs/201516/guidelines/2015-16_perf_env_guidelines.pdf

Canadian Association of Community Television Users and Stations (CACTUS). (2015). CRTC 2015-421: A review of the policy framework for local and community television programming. Intervention \#1472.

Canadian Media Guild (CMG) (2013a) Workers in Canadian factual TV are not 'living the dream' (Rep.).

Canadian Media Guild (CMG) (2013b) Summary of key results of CMG's factual survey 2013 (Rep.).

Canadian Heritage, (2017). Creative Canada policy framework. Catalogue No. CH4185/2017E-PDF. Retrieved https://www.canada.ca/en/canadianheritage/campaigns/creative-canada/framework.html

Canadian Radio-television and Communications Commission (CRTC). (1999, 17 May). 
Broadcasting Public Notice CRTC 1999-84: New media.

Canadian Radio-television and Communications Commission (CRTC). (2008, January

11). A Competitive Balance for the communications industry: Submission of the

Canadian Radio-Television and Telecommunications Commission to the

Competition Policy Review Panel. CRTC. Retrieved from

http://www.crtc.gc.ca/eng/publications/reports/cprp.htm

Canadian Radio-television and Communications Commission (CRTC). (2009, June 4).

News Release: CRTC extends exemption for new media and calls for a national digital strategy. Retrieved from

http://www.crtc.gc.ca/eng/com100/2009/r090604.htm

Canadian Radio-television and Communications Commission (CRTC). (2015 March 12).

Broadcasting Regulatory Policy CRTC 2015-86. The way forward : Creating compelling and diverse Canadian programming.

Canadian Radio-television and Communications Commission (CRTC). (2016 June 15). Broadcasting Regulatory Policy CRTC 2016-224: Policy framework for local and community television.

Canadian Radio-television and Communications Commission (CRTC). (2016 August 25). Broadcasting Regulatory Policy CRTC 2016-343: Policy framework for Certified Independent Production Funds.

Canadian Radio-television and Communications Commission (CRTC). (2017 May 15). Broadcasting Decision CRTC 2017-148: Renewal of licences for the television services of large English-language ownership groups - Introductory decision.

Caro, I.N. (2014 August 14). What is CBC Punchline and why is it so terrible? Retrieved http://o.canada.com/entertainment/cbc-punchline-what-the-hell-is-this-thing499651

Carr, D. (2013, February 24). For "House of Cards," Using Big Data to Guarantee Its Popularity. The New York Times. Retrieved from http://www.nytimes.com/2013/02/25/business/media/for-house-of-cards-using-bigdata-to-guarantee-its-popularity.html

Carsrud A, Brännback M (2011) Entrepreneurial motivations: what do we still need to know? Journal of Small Business Management, 49, 9-26. 
Carsrud, A., Brännback, M., Elfving, J., and Brandt, K. (2009). Motivations: The entrepreneurial mind and behavior, in A. Carsrud and M. Brännback (Eds.). Understanding the entrepreneurial mind: Opening the black box. Heidelberg: Springer, 141-166.

Cassar, G. (2007), Money, money, money? A longitudinal investigation of entrepreneurial career reasons, growth preferences and achieved growth. Entrepreneurship and Regional Development, 19(1), 89-107.

Christian, A. (2012a). Off the line: Independent television and the pitch to reinvent Hollywood (Doctoral dissertation). University of Pennsylvania.

Christian, A. J. (2012b). Beyond big video: The instability of independent networks in a new media market. Continuum, 26(1), 73-87.

Christian, A. J. (2012c). The web as television reimagined? Online networks and the pursuit of legacy media. Journal of Communication Inquiry, 36(4), 340-356.

Christian, A. J. (2014). Indie TV: Innovations in series development. In Bennett, J., \& Strange, N. Ed. Media Independence: Working with Freedom Or Working for Free? (Vol. 69). Routledge.

Christopherson, S. (2008). Beyond the Self-expressive Creative Worker An Industry Perspective on Entertainment Media. Theory, Culture \& Society, 25(7-8), 73-95.

Christopherson, S. (2009). Working in the creative economy: Risk, adaptation, and the persistence of exclusionary networks. In A. McKinlay \& C. Smith (Eds.), Creative labour: Working in the creative industries (pp. 73-95). Palgrave Macmillan.

Conor, B. (2014). Screenwriting: Creative Labor and Professional Practice. Routledge.

Cook, K. (2008). In-Depth Interview. In Lisa M. Given (Ed.), The Sage Encyclopedia of Qualitative Research Methods. (pp. 423-424). Thousand Oaks, CA: SAGE Publications, Inc. doi: http://dx.doi.org/10.4135/9781412963909.n209

Cooper, M. (2005). Hyper-commercialism and the media: The threat to journalism and democratic discourse. In Skinner, D., Compton, J. R., \& Gasher, M. (Eds.). (2005). Converging media, diverging politics: A political economy of news media in the United States and Canada. Lexington Books.

Coutanche, M., and Davis, C. (2013). The 2012 report on Canadian English-language 
screenwriters. Toronto: RTA School of Media.

Coutanche, M., Davis, C. H., \& Zboralska, E. (2015). Telling Our Stories: Screenwriters and the Production of Screen-Based Culture in English-Speaking Canada. Canadian Journal of Communication, 40(2).

Crang, M. \& Cook, I. (2007) Participant Observation. In Crang, M. \& Cook, I. (Eds.), Doing Ethnographies. (pp. 36-60). London, England: SAGE Publications Ltd. doi: http://dx.doi.org/10.4135/9781849208949.n4

Creative Skillset (2015). The creative media workforce survey 2014 summary report. Creative Skillset. Retrieved: https://creativeskillset.org/assets/0001/0465/Creative_Skillset_Creative_Media_Wo rkforce_Survey_2014.pdf

Crenshaw, K. (1989). Demarginalizing the intersection of race and sex: A black feminist critique of antidiscrimination doctrine, feminist theory and antiracist politics. U. Chi. Legal F., 139.

Croteau, D., \& Hoynes, W. (2001). The business of media: Corporate media and the public interest. Pine Forge Press.

Chazan, M., Helps, L., Stanley, A., \& Thakkar, S. (Eds.). (2011). Home and native land: Unsettling multiculturalism in Canada. Between the Lines.

Couldry, N. (2011). More sociology, more culture, more politics: or, a modest proposal for 'convergence' studies. Cultural Studies, 25(4-5), 487-501.

Cummins, J. (2016 April 1). CMF sets program budget for 2016/17 at 371.2 million. Retrieved http://playbackonline.ca/2016/04/01/cmf-sets-program-budget-for201617-at-371-2m/

Cunningham, S., \& Silver, J. (2013). Screen distribution and the new King Kongs of the online world. Palgrave Macmillan.

Cunningham, S., \& Craig, D. (2016). Online entertainment: A new wave of media globalization?. International Journal of Communication, 10, 5409-5425.

Cunningham, S., \& Craig, D. (2017). Being 'really real' on YouTube: Authenticity, community and brand culture in social media entertainment. Media International 
Australia, $1329878 \times 17709098$.

Cunningham, S., Craig, D., \& Silver, J. (2016). YouTube, multichannel networks and the accelerated evolution of the new screen ecology. Convergence, 22(4), 376-391.

Curran, J., \& Blackburn, R. (2000). Researching the small enterprise. London: Sage.

Curtin, M., Holt, J., \& Sanson, K. (Eds.) (2014). Distribution revolution: Conversations about the digital future of film and television. University of California Press.

Davis, C., Creutzberg, T., \& Arthurs, D. (2009). Applying an innovation cluster framework to a creative industry: The case of screen-based media in Ontario. Innovation, 11(2), 201-214.

Davis, C., Shtern, J., Coutanche, M and Godo, E. (2014). Screenwriters in Toronto: Centre, Periphery, and Exclusionary Networks in Canadian Screen Storytelling, pp. 77-98 in J. Grant (ed.), Seeking Talent for Creative Cities: the Social Dynamics of Innovation. Toronto: University of Toronto Press.

Davis, H., and Scase, R. (2001). Managing creativity. Brunner-Routledge.

Dawson, C., \& Henley, A. (2012). "Push" versus "pull" entrepreneurship: an ambiguous distinction?. International Journal of Entrepreneurial Behavior \& Research, 18(6), 697-719.

Defy Media (2015). Acumen report: Youth video diet. Retrieved from http://cdn.defymedia.com/wp-content/uploads/2016/04/DEFY_MEDIA-AcumenYouth_Video_Diet.pdf

De Rosa, M., \& Burgess, M. (2017). Exporting Canadian television globally: Trends, opportunities and future Directions. Canadian Media Producers Association. (Rep.).

Dess, G.G., Lumpkin, G.T., \& Covin, J.G. (1997). Entrepreneurial strategy making and firm performance: Tests of contingency and configurational models. Strategic Management Journal, 18: 677-695.

Ding Y, Du Y, Hu Y, et al. (2011) Broadcast yourself: Understanding YouTube uploaders. In: ACM SIGCOMM internet measurement conference (IMC Digital Library), pp.361-370.

Douglas, E.J. and Shepherd, D.A. (2002). Self-employment as a career choice: 
attitudes, entrepreneurial intentions, and utility maximization. Entrepreneurship Theory and Practice, 26(3), 81-90.

Duopoly (2015). Content everywhere (2): Securing Canada's place in the digital future (Rep.). Retrieved https://gallery.mailchimp.com/26f1366ec639a5eb1954790e1/files/ContentEverywh ere2_2015_03_02.pdf

Duopoly. (2016). Rep. Strengthening the Business: Capitalizing Canada's Content Business. Retrieved http://trends.cmffmc.ca/media/uploads/reports/Strengthening-the-Business-_Capitalizing-CanadaContent-Business.pdf

Earle, J.S. \& Sakova, Z. (2000). Business start-ups or disguised unemployment? Evidence on the character of self-employment from transition economies. Labour Economics, 7(5), 575-601.

Edelman, L., Brush, C., Manolova, T., \& Greene P. (2010). Start-up motivations and growth intentions of minority nascent entrepreneurs. Journal of Small Business Management. 48,174-196.

Edwards, J. (2014). Yes, you can make six figures as a YouTube star ... and still end up poor. Retrieved from http://www.businessinsider.com/how-much-money-youtubestars-actually-make-2014-2

Eikhof, D. R., \& Warhurst, C. (2013). The Promised Land? Why social inequalities are systemic in the creative industries. Employee Relations, 35(5), 3-3.

Elfving, J., Brannback, M., \& Carsrud, A. (2009). Toward a contextual model of entrepreneurial intentions. In Carsrud, A., and Brannback, M. (Eds.). Understanding the entrepreneurial mind: Opening the black box. Heidelberg: Springer, 23-34.

Elo, S., \& Kyngas, H. (2008). The qualitative content analysis process. Journal of Advanced Nursing, 62(1), 107-115.

Employment and Social Development Canada (ESDC) (2015). Employment Equity Act Annual Report. (Rep.). Retrieved. https://www.canada.ca/en/employment-socialdevelopment/services/labour-standards/reports/employment-equity-2015.html\#a9

Evans, G. (2009). Creative cities, creative spaces and urban policy. Urban studies, 46(5- 

6), $1003-1040$.

Fairlie, R. W. (2005). Entrepreneurship among disadvantaged groups: An analysis of the dynamics of self-employment by gender, race and education. In Handbook of entrepreneurship, 2, 437-478.

Fairlie, R. W., \& Meyer, B. D. (1996). Ethnic and racial self-employment differences and possible explanations. Journal of Human Resources, 757-793.

Fielden, S. L., \& Dawe, A. (2004). Entrepreneurship and social inclusion. Women in Management Review, 19(3), 139-142.

Fiske, J. (2002). Television culture. Routledge.

Fleras, A. (2011). The Media Gaze: Representations of Diversities in Canada. UBC Press.

Flick, U. (2014). Qualitative content analysis. In Flick, U. The SAGE handbook of qualitative data analysis (pp. 170-183). London: SAGE Publications, Ltd doi: 10.4135/9781446282243.n

Franzosi, R. (2004). Content Analysis. In M. Hardy, \& A. Bryman (Eds.), Handbook of Data Analysis. (pp. 548-566). London, England: SAGE Publications, Ltd. doi: http://dx.doi.org/10.4135/9781848608184.n24

Frau-Meigs, D. (2013). Unesco/ WSIS Report 2013: Exploring the evolving mediascape: Towards updating strategies to face challenges and seize opportunities. Unesco/ WSIS.

Freidson, E. (1990). Labors of love: A prospectus. In: Erikson, K., Vallas, S.P. (Eds.), The nature of work: Sociological perspectives. Yale University Press.

Fuchs, C. (2017). Social media: A critical introduction. London: SAGE Publications, Ltd.

Garnham, N. (1995). Political economy and cultural studies: Reconciliation or divorce?. Critical Studies in Mass Communication, 12, 62-62.

Garnham, N. (2011). The political economy of communication revisited. In Wasko, J., Murdock, G., \& Sousa, H. (Eds.). (2011). The handbook of political economy of communications. Wiley. 
Geist, M. (2007). Canadian broadcasting policy for a world of abundance. Canadian Issues. Winter.

Giacomin, O., Janssen, F., Guyot, J. L., \& Lohest, O. (2011). Opportunity and/or necessity entrepreneurship? The impact of the socio-economic characteristics of entrepreneurs. MPRA Paper, 29506, 1-42.

Gill, R. (2011). Life is a pitch: Managing the self in new media work. In: M. Deuze, (Eds.). Managing media work (pp. 249-263). London: Sage.

Gillespie, T. (2010). The politics of 'platforms'. New Media \& Society, 12(3), 347-364.

Gitlin, T. (1983). Inside prime time. University of California Press.

Given, L. M. (2008). The SAGE encyclopedia of qualitative research methods Thousand Oaks, CA: SAGE Publications Ltd.

Golding, P. \& Murdock, G. (1996) Culture, communications, and political economy. In Mass media and society. James Curran and Michael Gurevitch (eds.) London: Arnold. pp. 60-83.

Goodman, H. (2001). In-Depth Interviews. In Bruce A. Thyer (Ed.), The handbook of Social Work Research Methods. (pp. 308-320). Thousand Oaks, CA: SAGE Publications, Inc. doi: http://dx.doi.org/10.4135/9781412986182.n17

Grant, P.S., \& Wood, C. (2004). Blockbusters and trade wars: Popular culture in a globalized world. Vancouver: Douglas \& McIntyre.

Grant, P. S. (2008, February 22). Reinventing the cultural tool kit: Canadian content on new media. In CCFTPA "Prime Time in Ottawa" Conference. Retrieved from http://www.mccarthy.ca/presentation_detail.aspx?id=3957

Green, H. (2015). The $\$ 1000$ CPM. Retrieved from https://medium.com/@hankgreen/the-1-000-cpm-f92717506a4b

Grossberg, L. (1995). Cultural studies vs. political economy: Is anybody else bored with this debate?.

Grugulis, I., \& Stoyanova, D. (2012). Social capital and networks in film and TV: Jobs for the boys?. Organization Studies, 33(10), 1311-1331. 
Hallinan, B., \& Striphas, T. (2014). Recommended for you: The Netflix Prize and the production of algorithmic culture. New Media \& Society. 1461444814538646.

Hardy, J. (2014). Critical political economy of the media: an introduction. Routledge.

Harper, S. (2015). Tweet, 7:19 PM - 5 August, https://twitter.com/pmharper/status/629054056678363136.

Harvey, A. M. (2005). Becoming entrepreneurs: Intersections of race, class, and gender at the black beauty salon. Gender \& Society, 19(6), 789-808.

Havens, T., Lotz, A. D., \& Tinic, S. (2009). Critical media industry studies: A research approach. Communication, Culture \& Critique, 2(2), 234-253.

Herman, E. S. \& McChesney, R.W., (1997). The global media: The new missionaries of corporate capitalism. London: Cassell.

Herman, E. S., \& Chomsky, N. (2008). Manufacturing consent: The political economy of the mass media. Random House.

Hesmondhalgh, D. (2010). Media industry studies, media production studies. In Curran, J. ed., Media and society. Bloomsbury Publishing.

Hesmondhalgh, D., and Baker, S. (2011). Creative labor: Media work in three cultural industries. Routledge.

Hickling, Arthurs, Low (2010). Rep. Ontario's Creative Cluster Study - Music, Film and TV - Magazines - Books - Interactive Digital Media. Retrieved http://www.mtc.gov.on.ca/en/publications/Creative_Cluster_Study.pdf

Hisrich, R.D. and Brush, C.G. (1986). The woman entrepreneur: Starting, managing, and financing a successful new business. Lexington Books, Lexington, MA.

Hoag, A. \& Compaine, B. (2006) Media entrepreneurship in the era of big media:

Prospects for new entrants. Unpublished manuscript, University of Pennsylvania.

Holgate, J., \& Mckay, S. (2007). Institutional barriers to recruitment and employment in the audio-visual industries. London: Working Lives Research Institute.

Holiday, R. (2014). Growth hacker marketing: a primer on the future of PR, marketing, 
and advertising. Portfolio.

Holt, J., \& Perren, A. (2009). Introduction: Does the world really need one more field of study? In Holt, J., \& Perren, A. (Eds.) Media industries: History, theory, and method. West Sussex, United Kingdom: Wiley-Blackwell.

Hughes, K. (2003). Pushed or pulled? Women's entry into self-employment and small business ownership. Gender, Work and Organization 10 (4), 433-454.

Hunt, D., Ramon, A. \& Tran, M. (2016). 2016 Hollywood diversity report: Busine $\$ \$$ as usual?. (Rep.). Ralph J. Bunche Center for African American Studies at UCLA.

Hunter, L. (2017 May 3). Rogers Community TV shutters more stations. Retrieved: https://cartt.ca/article/rogers-community-tv-shutters-more-stations

Independent Broadcast Group (IBG) (2016 November 15). Canadian content in a digital world: The role of independent broadcasters as content creators and providers. Retrieved http://www.canadiancontentconsultations.ca/system/file_answers/files/63d30c48a1 b2b38fc0032ce25f06c3d7fdab39f8/001/420/953/original/IBGGDI_Submission_re_Canadian_Content_in_a_Digital_World.pdf?1480110034

Independent Production Fund (IPF). 2013. Independent Production Fund annual report 2013. (Rep.) Retrieved http://ipf.ca/AR2013/

Independent Production Fund (IPF). (2017). Web Drama Series Program guidelines. Retrieved https://ipf.ca/guidelines/

Independent Production Fund (IPF). (n.d.). Frequently Asked Questions. Retrieved 17 May 2017 from https://ipf.ca/resources/faqs/

Independent Web Creators of Canada (IWCC). (2015). CRTC 2015-421: A review of the policy framework for local and community television programming. Intervention \#1415

Jackson, E. (2017 August 14). Cabinet orders CRTC to review decision to decrease spending on Canadian TV programs. Retrieved http://business.financialpost.com/telecom/cabinet-orders-crtc-to-review-decisionto-decrease-spending-on-canadian-tv-programs 
Jennings, J. E., Jennings, P. D., \& Sharifian, M. (2014). Living the dream? Assessing the "Entrepreneurship as Emancipation" perspective in a developed region.

Entrepreneurship Theory and Practice.

Johnson, J. (2001). 5 In-Depth Interviewing. In Jaber F. Gubrium, \& James A. Holstein (Eds.), Handbook of Interview Research. (pp. 103-120). Thousand Oaks, CA: SAGE Publications, Inc. doi: http://dx.doi.org/10.4135/9781412973588.d8

Joly, M. (2017, September 28). Launch of Creative Canada speech by Honourable Melanie Joly. Speech presented in Ottawa. Retrieved https://www.canada.ca/en/canadian-heritage/news/2017/09/creative_canada_avisionforcanadascreativeindustries.html

Jones, C. (2002). Signaling expertise. How signals shape careers in creative industries. In Peiperl, M., Arthur, M. B., \& Anand, N. (Eds.). Career creativity: Explorations in the remaking of work. Oxford University Press on Demand.

Jorgensen, D.L.(1989). The methodology of participant observation. In Jorgensen D.L. (Ed.), Participant observation. (pp. 12-26). Thousand Oaks, CA: SAGE Publications, Inc. doi: http://dx.doi.org/10.4135/9781412985376.n1

Kane, L. (9 December 2014). YouTube stars Lilly Singh and Megan MacKay a new breed of celebrity. The Canadian Press.

http://thechronicleherald.ca/artslife/1256543-youtube-stars-lilly-singh-and-meganmackay-a-new-breed-of-celebrity

Kanter, R. M. 1982. The middle manager as innovator. Harvard Business Review, 60(4): 95- 106.

Kaye, J., \& Davis, C. H. (2011). If it ain't on the page, it ain't on the stage: screenwriting, national specificity and the English-Canadian feature film. Journal of Screenwriting, 2(1), 61-83.

Kellner, D. (2001). Cultural studies and social theory: a critical intervention. Handbook of Social Theory, 395-409.

Kellner, D. (2009). Media industries, political economy, and media/ cultural studies: An articulation. In Holt, J., \& Perren, A. (Eds.) Media industries: History, theory, and method. West Sussex, United Kingdom: Wiley-Blackwell.

Kelty, C. (2013). From participation to power. In Delwiche, A., \& Henderson, J. J. (Eds.), 
The participatory cultures handbook, pp. 22-33. New York: Routledge.

Klarer, M. (2014). Putting television 'aside': Novel narration in House of Cards. New Review of Film and Television Studies, (forthcoming), 1-18.

Kolb, B. (2008). In-depth, Intercept and Expert Interviews. In Marketing research. (pp. 141-158). London, England: SAGE Publications Ltd. doi: http://dx.doi.org/10.4135/9780857028013.d137

Krippendorff, K. (1980). Content analysis. An Introduction to its methodology. Beverly Hills: Sage.

Kris, E., Kurz, O. (1987). L'image de l'artiste. French translation. Editions Rivages, Marseille.

Kymlicka, W. (1995). Multicultural citizenship: A liberal theory of minority rights. Clarendon Press.

Lashley, M. (2012). Making culture on YouTube: Case studies of cultural production on the popular web platform. (Doctoral dissertation). University of Georgia.

Lee, D. (2011). Networks, cultural capital and creative labour in the British independent television industry. Media, Culture \& Society, 33(4), 549-565.

Lister, R. (2004). Inequality, social divisions and the differential experience of poverty. In Poverty. Cambridge: Polity.

Lotz, A. D. (2009). Industry-level studies and the contributions of Gitlin's Inside Prime Time. In Mayer, V., Banks, M. J., \& Caldwell, J. T. (Eds.). Production studies: cultural studies of media industries. Routledge.

Making Media Public and the Communications Policy Working Group (2015). CRTC 2015-421: A review of the policy framework for local and community television programming. Intervention \#1441.

Maloney, V. (2017 May 25). CBC reveals plans for new OTT service. Retrieved: http://playbackonline.ca/2017/05/25/cbc-goes-for-the-x-factor-with-ott-service/

Marks, D. F. \& Yardley, L. (2004). Content and thematic analysis. In Research methods for clinical and health psychology (pp. 56-68). : SAGE Publications Ltd. 
Marom, D., Robb, A., \& Sade, O. (2016). Gender dynamics in crowdfunding (Kickstarter): evidence on entrepreneurs, investors, deals and taste-based discrimination.

Marx, K. (2010). A contribution to the critique of political economy. In Marx today (pp. 91-94). Palgrave Macmillan US.

Mason, C. (1989). Explaining recent trends in new firm formation in the UK: Some evidence from South Hampshire. Regional Studies, 23(4), 31-46.

Mayer, V. (2011). Below the line: Producers and production studies in the new television economy. Duke University Press.

Mayer, V., Banks, M. J., \& Caldwell, J. T. (2009). Introduction: Production studies: Routes and routes. In Mayer, V., Banks, M. J., \& Caldwell, J. T. (Eds.). Production studies: cultural studies of media industries. Routledge.

McBride, J. (2017 March 20). YouTube restricting LGBT content: 5 fast facts you need to know. Retrieved from

http://heavy.com/news/2017/03/youtube-you-tube-lgbt-content-videos-restrictedmode-gay-restricting-is/

McChesney, R. (2008). The political economy of media: Enduring Issues, Emerging Dilemmas. New York: Monthly Review Press.

McDonald, S., \& Day, J. C. (2010). Race, gender, and the invisible hand of social capital. Sociology Compass, 4(7), 532-543.

McLeod, C., O'Donohoe, S., \& Townley, B. (2009). The elephant in the room? Class and creative careers in British advertising agencies. Human Relations, 62(7), 1011 1039.

McPherson, M., Smith-Lovin, L., \& Cook, J. M. (2001). Birds of a feather: Homophily in social networks. Annual Review of Sociology, 27(1), 415-444.

McRobbie, A. (2004) 'Everyone is creative': Artists as pioneers of the new economy? In Bennett, T. and Silva, E.B. (Eds.). Contemporary culture and everyday life (pp.186201). London: Sociology Press.

Media Technology Monitor (MTM). (2016, February 4). Media technology adoption 
2015: Analysis of the English-language market. (Rep.).

Meehan, E. R. (1999). Commodity, culture, common sense: Media research and paradigm dialogue. Journal of Media Economics, 12(2), 149-163.

Meehan, E. R. (2006). Gendering the commodity audience: Critical media research, feminism, and political economy. Media and Cultural Studies, 311.

Meehan, E., and Torre, P. (2011). Markets in theory and markets in television. In Wasko, J., Murdock, G., \& Sousa, H. (Eds.). The handbook of political economy of communications. Wiley.

Middleton, C. (2011). Canada's telecommunications policy environment. Telecommunications Journal of Australia, 61(4), pp. 69.1-69.14

Mills, R. (2012). Independent Web Creators Manifesto. Retrieved http://www.robbomills.net/indiewebcreatemanifesto.html

Morgan, R. E., \& Strong, C. A. (2003). Business performance and dimensions of strategic orientation. Journal of Business Research, 56(3), 163-176.

Mosco, V. (2004). The digital sublime. Myth, power, and cyberspace. Cambridge, MA: The MIT Press.

Mosco, V. (2009). The political economy of communication. 2nd ed. Thousand Oaks: Sage Publications.

Mostrous, A. (2017, February 9). Big brands fund terror through online adverts. Retrieved April 26, 2017, from https://www.thetimes.co.uk/article/big-brands-fundterror-knnxfgb98

Müller, E. (2009). Where quality matters: discourses on the art of making a YouTube video. In In: P. Snickers \& P. Vonderau (eds.), The YouTube reader. National Library of Sweden, Stockholm, pp. 126-139.

Murray, C. (2002). Silent on the set: Cultural diversity and race in English Canadian TV drama. Strategic Research and Analysis, 63.

Murray, C (2009) Designing monitoring to promote cultural diversification in TV. Canadian Journal of Communication, 34(4). 
Nabi, A. Walmsley \& Holden, R. (2015) Pushed or pulled? Exploring the factors underpinning graduate start-ups and non-start-ups. Journal of Education and Work, 28(5), 481-506.

Nordicity. (2014). Industry profile of the independent web series creators of Ontario. (Rep). Retrieved http://www.iwcc-ciwc.org/wp-content/uploads/2014/06/IWCCIndustry-Profile_Final-Report-June-23.pdf

Oakley, K. (2006). Include us out-economic development and social policy in the creative industries. Cultural Trends, 15(4), 255-273.

Oakley, K. (2013). Absentee workers: Representation and participation in the cultural industries. In Banks, M., Gill, R., \& Taylor, S. (Eds.). Theorizing cultural work: Labour, continuity and change in the cultural and creative industries. Routledge.

Oliviera, M. (December 13, 2012). YouTube wants to make a star out of you. The Canadian Press. http://www.metronews.ca/entertainment/2012/12/13/youtubewants-to-make-a-star-out-of-you.html

Ontario Media Development Corporation (OMDC). (2017). OMDC Interactive Digital Media Fund Production and Concept Definition Programs Retrieved: http://www.omdc.on.ca/Assets/Industry+Initiatives/English/Content+and+Marketin g/IDM+Fund/OMDC+IDM+Fund-Guidelines_en.pdf

Orhan, M. \&Scott, D. (2001). Why women enter into entrepreneurship: an explanatory model. Women in Management Review, 16 (5), 232-43.

Parker, M. (2017, October 3). Commentary: Why Mélanie Joly's Creative Canada is good news for Canadian screenwriters and Canadian culture. Retrieved https://cartt.ca/article/commentary-why-mélanie-joly's-creative-canada-good-newscanadian-screenwriters-and-canadian

Patreon (2017a). Terms of use. Retrieved: https://www.patreon.com/legal

Patreon (2017b). Jason Leaver is creating Out With Dad. Retrieved https://www.patreon.com/OutWithDad

Payne, G., \& Payne, J. (2004). Participant observation. In The SAGE Key Concepts series: Key concepts in social research. (pp. 166-171). London, England: SAGE Publications, Ltd. doi: http://dx.doi.org/10.4135/9781849209397.n35 
Perlin, R. (2012). Intern nation: How to earn nothing and learn little in the brave new economy. Verso Books.

Peters, T. (1997) The Circle of Innovation. London: Hodder and Stoughton.

Peterson, R. A. (1990). "Why 1955? Explaining the advent of rock music." Popular Music 9, 97-116.

Picard, R.G., Davis, C.H., Papandrea, F., \& Park, S. (2016). Platform proliferation and its implications for domestic content policies. Informatics and Telematics 33: 683-692.

Pinto, J. (2017 March 8). Online-only projects now eligible for CAVCO tax credits. Retrieved: http://playbackonline.ca/2017/03/08/online-only-projects-now-eligiblefor-tax-credits/\#ixzz4jxBOuov9

Playback, (2017). Tweet. 11:48 AM - 2 February, https://twitter.com/PlaybackOnline/status/827181945947877377

Pogey Beach. (2016). Kickstarter. Retrieved 7 June 2017, from https://www.kickstarter.com/projects/698586302/pogey-beach

Powdermaker, H. (1950). Hollywood the dream factory. New York: Little, Brown and Company.

Pozarny, P. (2016). Gender roles and opportunities for women in urban environments.

Quail, C. (2012). Television goes online: Myths and realities in the contemporary context. Global Media Journal, 12(20).

Raboy, M. (1990). Missed opportunities: The story of Canada's broadcasting policy. Montreal \& Kingston: McGill-Queen's University Press.

Raj, A. (2014, September 08). CRTC chief talks Netflix, Shomi and the future of TV. Retrieved from http://www.huffingtonpost.ca/2014/09/08/crtc-netflix-shomi-jeanpierre-blais_n_5774894.html

Rath, J. (2017a, March 23.). Here are the biggest brands that have pulled their advertising from YouTube over extremist videos. Retrieved April 26, 2017, from http://www.businessinsider.com/these-brands-pulled-ads-from-youtube-andgoogle-over-extremist-content-2017-3 
Rath, J. (2017b, March 27). Analysts predict the YouTube advertiser boycott will cost Google $\$ 750$ million. Retrieved April 26, 2017, from http://www.businessinsider.com/analyst-predicts-the-youtube-ad-boycott-will-costgoogle-750-million-2017-3

Reid, R. (2016 August 31). CRTC's CIPF points change sparks controversy. Retrieved http://playbackonline.ca/2016/08/31/creatives-rally-against-crtcs-cipf-pointschange/

Reid, R. (2017 May 17). Unions, guilds react to CRTC licensing decision. Retrieved http://playbackonline.ca/2017/05/17/unions-guilds-react-to-crtc-licensing-decision/

Reid, R. (n.d.). Creative Canada leaves many questions unanswered. Retrieved http://playbackonline.ca/2017/09/29/creative-canada-leaves-many-questionsunanswered/?utm_source=newsletter\&utm_medium=email\&utm_campaign=creati ve-canada-leaves-many-questions-unanswered\&_u=TDLCuBEvZ4A\%3d

Reynolds, P.D., Camp, S.M., Bygrave, W.D., Autio, E. \& Hay, M. (2002). GEM Global Entrepreneurship Report, 2001 Summary Report, 1-126. Accessed June 6, 2016 http://www.gemconsortium.org/download/1293023015823/Summary\%20Report\% 20Version \%208\%2002\%20April\%2002.pdf

Riffe, D., Lacy, S., \& Fico, F. (2005). Analyzing media messages: Using quantitative content analysis in research. Routledge.

Rindova, V., Barry, D., \& Ketchen, D.J. (2009). Entrepreneuring as emancipation. Academy of Management Review. 34(3), 477-491.

Robertson, A. (2016, September 1). Why is YouTube being accused of censoring vloggers? Retrieved April 26, 2017, from

http://www.theverge.com/2016/9/1/12753108/youtube-is-over-party-advertisingmonetization-censorship

Robichaud, Y., LeBrasseur, R., Riverin, N. \& Zinger, J.T. (2006). L'influence des motivations pression-attraction (push-pull) sur la conduite d'une petite entreprise lors de la phase de création: Une comparaison hommes/femmes. Presented at Congrès International Francophone en Entrepreneuriat et PME (CIFEPME), Fribourg (Switzerland), 1- 19. 
Romano, A. (2016, September 2). YouTube's "ad-friendly" content policy may push one of its biggest stars off the website. Retrieved April 26, 2017, from http://www.vox.com/2016/9/2/12746450/youtube-monetization-phil-defrancoleaving-site

Ross, A. (2006). Nice work if you can get it: The mercurial career of creative industries policy. Work Organisation, Labour and Globalisation, 1(1), 13-30.

Rosten, L.C. (1941). Hollywood: the movie colony, the movie makers. New York: Harcourt, Brace and Company.

Saha, M. (n.d.). How much money do YouTubers make? Answered by YouTubers. Retrieved from http://techwiser.com/how-much-can-you-make-from-youtube/

Schiller, D. (2000). Digital capitalism: Networking the global market system. MIT press.

Shields, M. (2016, August 29). Kimberly-Clark scores hit video series with "Carmilla." Wall Street Journal. Retrieved from http://www.wsj.com/articles/kimberly-clarkscores-hit-video-series-with-carmilla-1472464800

Shirky, C. (2008). Here comes everybody. London: Penguin.

Siebert, S., \& Wilson, F. (2013). All work and no pay: consequences of unpaid work in the creative industries. Work, Employment \& Society.

Skilton, P. F. (2008). Similarity, familiarity and access to elite work in Hollywood: Employer and employee characteristics in breakthrough employment. Human Relations, 61(12), 1743-1773.

Skinner, D., and Gasher, M. (2005). So much by so few: Media policy and ownership in Canada. In Skinner, D., Compton, J. R., \& Gasher, M. (Eds.). (2005). Converging media, diverging politics: A political economy of news media in the United States and Canada. Lexington Books.

Skinner, D. (2008). Broadcasting in Canada. In David Ward and Anthony McNicholas (Eds.). Television and public policy: Change and continuity in an era of liberation (pp. 3-26). New Jersey: Lawrence Erlbaum.

Smith, R., \& Air, C. (2012). No choices, no chances: how contemporary enterprise 
culture is failing Britain's underclasses. The International Journal of Entrepreneurship and Innovation, 13(2), 103-113.

Smythe, D. 1981. Dependency road: Communications, capitalism, consciousness and Canada. Norwood, N.J.: Ablex Publishing Corporation.

Solon, O. (2017, March 21). I can't trust YouTube anymore: Creators speak out in Google advertising row. Retrieved from https://www.theguardian.com/technology/2017/mar/21/youtube-googleadvertising-policies-controversial-content

Solutions Research Group. (2003, December). Cultural diversity on television phase V research: Benchmark content analysis. Toronto, ON: Solutions Research Group.

Spangler, T. (2016, September 1). YouTube Slammed by Creators Over "Ad Inappropriate" Content Policy, Which Google Says Hasn't Changed. Retrieved April 26, 2017, from http://variety.com/2016/digital/news/youtube-slammed-bycreators-about-pulling-ads-from-inappropriate-content-1201850250/

Standing Committee on Heritage. (2017). Disruption: Change and churning in Canada's media landscape (Rep.) 42 $2^{\text {nd }}$ Parliament, $1^{\text {st }}$ Session.

Statistics Canada (2013). Immigration and ethnocultural diversity in Canada. (Rep.) Retrieved: https://www12.statcan.gc.ca/nhs-enm/2011/as-sa/99-010-x/99-010x2011001-eng.cfm\#a4

Statistics Canada (2015). Visible minority of person. Retrieved: http://www23.statcan.gc.ca/imdb/p3Var.pl?Function=DECl\&ld=257515

Strangelove, M. (2010). Watching YouTube: Extraordinary videos by ordinary people. University of Toronto Press.

Tapscott, D., and Williams A.D. (2007). Wikinomics: How mass collaboration changes everything. New York: Penguin.

Teenagers. (2017). About. Retrieved 10 April 2017 from https://www.youtube.com/user/teenagersweb series/about

Telefilm. (2016a) Micro-Budget Production Program: Funding of micro-budget projects from emerging filmmakers. Retrieved https://telefilm.ca/wp- 
content/uploads/guidelinesmicro-budget-2017eng.pdf

Telefilm. (2016b). Micro-Budget Production Program: Frequently asked questions (FAQ). Retrieved: https://telefilm.ca/wp-content/uploads/faq-micro-budget-2017eng.pdf

Telefilm. (2016c). List of designated partners: Micro-Budget Production Program main component. Retrieved: https://telefilm.ca/wp-content/uploads/mb-list-partners-indliste-partenaires-autoc-2017-18.pdf

Telefilm. (2017). Original Canadian voices, original Canadian stories: Telefilm Canada and the Talent Fund announce the 18 projects selected under the Micro-Budget Production Program. Retrieved https://telefilm.ca/en/news-releases/originalcanadian-voices-original-canadian-stories-telefilm-canada-talent-fund-announce18-projects-selected-micro-budget-production-program

The Community Media Policy Working Group. (2015). CRTC 2015-421: A review of the policy framework for local and community television programming. Intervention \#1464.

Tinic, S. (2005). On location: Canada's television industry in a global market. Toronto: University of Toronto.

Toronto (2013). 2011 National Household Survey: Immigration, citizenship, place of birth, ethnicity, visible minorities, religion and Aboriginal peoples. City of Toronto. Retrieved:

https://www1.toronto.ca/city_of_toronto/social_development_finance_administrat ion/files/pdf/nhs_backgrounder.pdf

Toronto (2017). Toronto facts: Diversity. City of Toronto. Retrieved: http://www1.toronto.ca/wps/portal/contentonly?vgnextoid=dbe867b42d853410Vg nVCM10000071d60f89RCRD

van Dijck J (2013) The culture of connectivity: A critical history of social media. New York: Oxford University Press.

Verstraete, G. (2011). The politics of convergence: On the role of the mobile object. Cultural Studies, 25(4-5), 534-547.

Vonderau, P. (2009) Writers becoming users: YouTube hype and the writers' strike. In: 
P. Snickers \& P. Vonderau (eds.), The YouTube reader. National Library of Sweden, Stockholm, pp. 108-125.

Vonderau, P. (2016). The video bubble: Multichannel networks and the transformation of YouTube. Convergence, 22(4), 361-375.

Vipond, M. (2011). The mass media in Canada (4th ed.). Toronto: James Lorimer and Company Limited.

Wagner, J. (2005). Nascent necessity and opportunity entrepreneurs in Germany: Evidence from the Regional Entrepreneurship Monitor (REM). University of Lüneburg Working Paper Series in Economics, 10, 1-24.

Walker, J. E. (2009). The history of black business in America: Capitalism, race, entrepreneurship (Vol. 1). UNC Press Books.

Walker, R. (2012, June 28). On YouTube, amateur is the new pro. The New York Times. Retrieved http://www.nytimes.com/2012/07/01/magazine/onyoutube-amateur-isthe-new-pro.html?_r=1\&partner=rss\&emc $=r s s$

Warnecke, T. (2013a). Entrepreneurship and gender: An institutional perspective. Journal of Economic Issues, 47(2), 455-464.

Warnecke, T. (2013b). Targeting the 'invisible': Improving entrepreneurship opportunities for informal sector women. Research to Practice Policy Brief, (32).

Wasko, J., \& Erickson, M. (2009). The political economy of YouTube. In Snickars, P., \& Vonderau, P. (eds.). The YouTube reader. Stockholm: National Library of Sweden.

Wasko, J., Murdock, G., \& Sousa, H. (2011). Introduction. The political economy of communications: Core concerns and issues. In Wasko, J., Murdock, G., \& Sousa, H. (Eds.). The handbook of political economy of communications. Wiley.

Watson, L. (2017 March 17) YouTube's Restricted Mode Is hiding some LGBT content [update]. Retrieved from http://gizmodo.com/youtubes-restricted-mode-is-hidingsome-lgbt-content-1793382337

Wiklund, J., \& Shepherd, D. (2003). Knowledge-based resources, entrepreneurial orientation, and the performance of small and medium-sized businesses. Strategic Management Journal, 24, 1307-1314. 
Williams, C. (2008). Beyond necessity-driven versus opportunity-driven entrepreneurship: A study of informal entrepreneurs in England, Russia and Ukraine. The International Journal of Entrepreneurship and Innovation, 9(3), 157165.

Wing-Fai, L., Gill, R., \& Randle, K. (2015). Getting in, getting on, getting out? Women as career scramblers in the UK film and television industries. The Sociological Review, 63(S1), 50-65.

Winseck, D. (2002). Netscapes of power: Convergence, consolidation and power in the Canadian mediascape. Media, culture and society, 24: 795-819.

Winseck, D. (2008). The state of media ownership and media markets: Competition or concentration and why should we care?. Sociology Compass, 2(1), 34-47.

Winseck, D. (2010). Financialization and the "crisis of the media": The rise and fall of (some) media conglomerates in Canada. Canadian Journal of Communication, 35(3).

Winseck, D. (2011). The political economies of media and the transformation of the global media industries. In Winseck, D. R., \& Jin, D. Y. (Eds.). The political economies of media: The transformation of the global media industries. London: Bloomsbury Academic.

Wogan, P. (2004). Deep hanging out: reflections on fieldwork and multisited Andean ethnography. Identities: Global Studies in Culture and Power, 11(1), 129-139.

Women in Film and Television (WIFT) (2012) Framework II: Canada's Screen-based Workforce (Rep.). WIFT.

Women in View (WIV). (2012).Women in View on Screen 2011-2012 (Rep.). Toronto: Women in View.

Women in View (WIV). (2013).Women in View on TV 2013 (Rep.). Toronto: Women in View.

Women in View (WIV). (2014).Women in View on Screen 2014 (Rep.). Toronto: Women in View.

Women in View (WIV). (2015). Women in View on screen 2015 (Rep.). Toronto: Women in View. Retrieved http://womeninview.ca/wp-content/uploads/2016/01/Women- 


\section{In-View-On-Screen-2015.pdf}

Wreyford, N. (2015). Birds of a feather: Informal recruitment practices and gendered outcomes for screenwriting work in the UK film industry. The Sociological Review, 63(S1), 84-96.

Writers Guild of Canada (WGC). (2015). Independent Production Agreement CoveringFreelance Writers Of Theatrical Films Television Programs And Other Production. Retrieved http://www.wgc.ca/files/IPA\%202015-2017\%20final.pdf

Younkin, P., \& Kuppuswamy, V. (2017). The Colorblind Crowd? Founder Race and Performance in Crowdfunding. Management Science.

YouTube (2016a). About YouTube. Retrieved 1 December, 2016, from https://www.youtube.com/yt/about/.

YouTube (2016b). Unlock YouTube Space Toronto. Retrieved 2 December, 2016, from https://www.youtube.com/yt/space/toronto-unlock.html

YouTube (2016c). Creator Academy. Retrieved 2 December, 2016, from https://creatoracademy.youtube.com/page/education?hl=en\&utm_source=YouTub e\%20Marketing\&utm_medium=Creator\%2OHub\&utm_campaign=Hub

Youtube (2017a).Statistics. Retrieved 5 April 2017 from https://www.youtube.com/yt/press/statistics.html

Youtube (2017b). Lesson: Earn money with YouTube. Retrieved 5 April 2017 from https://creatoracademy.youtube.com/page/lesson/revenue-basics\#yt-creatorsstrategies-3

YouTube (2017c). YouTube partner program terms. Retrieved 20 April 2017 from https://youtube-creators.googleblog.com/2017/04/introducing-expandedyoutube-partner.html

YouTube (2017d). Advertiser-friendly content guidelines. Retrieved from https://support.google.com/youtube/answer/6162278?hl=en

YouTube (2017e). Disable or enable Restricted Mode. Retrieved from https://support.google.com/youtube/answer/174084?co=GENIE.Platform\%3DDes 
ktop\&hl=en

YouTube (2017f). Strengthening YouTube for advertisers and creators. Retrieved from https://youtube-creators.googleblog.com/2017/03/strengthening-youtube-foradvertisers.html

Zahra, S. A. (1991). Predictors and financial outcomes of corporate entrepreneurship: An explorative study. Journal of Business Venturing, 6, 259-285.

Zahra, S.A., \& Covin, J.G. (1993). Business strategy, technology policy and firm performance. Strategic Management Journal, (14), 451-478.

Zboralska, E. \& Davis, C. (2017). Transnational over-the-top media distribution as a business and policy disruptor: The case of Netflix in Canada. The Journal of Media Innovations, 4(1), 4-25.

Zboralska, E., Davis, C., Shtern, J., \& Ciccone, V. (2017). Cultural diversity reporting in the Canadian audiovisual industry: Making monitoring meaningful. Quaderns del CAC, 43(xx), 73-85.

Zboralska, E. (2017). No more status quo! Canadian web-series creators' entrepreneurial motives through a contextualized "Entrepreneuring as Emancipation" framework. International Journal on Media Management, 19(1), 2953. 